\title{
Vergleich forstlicher Managementstrategien
}

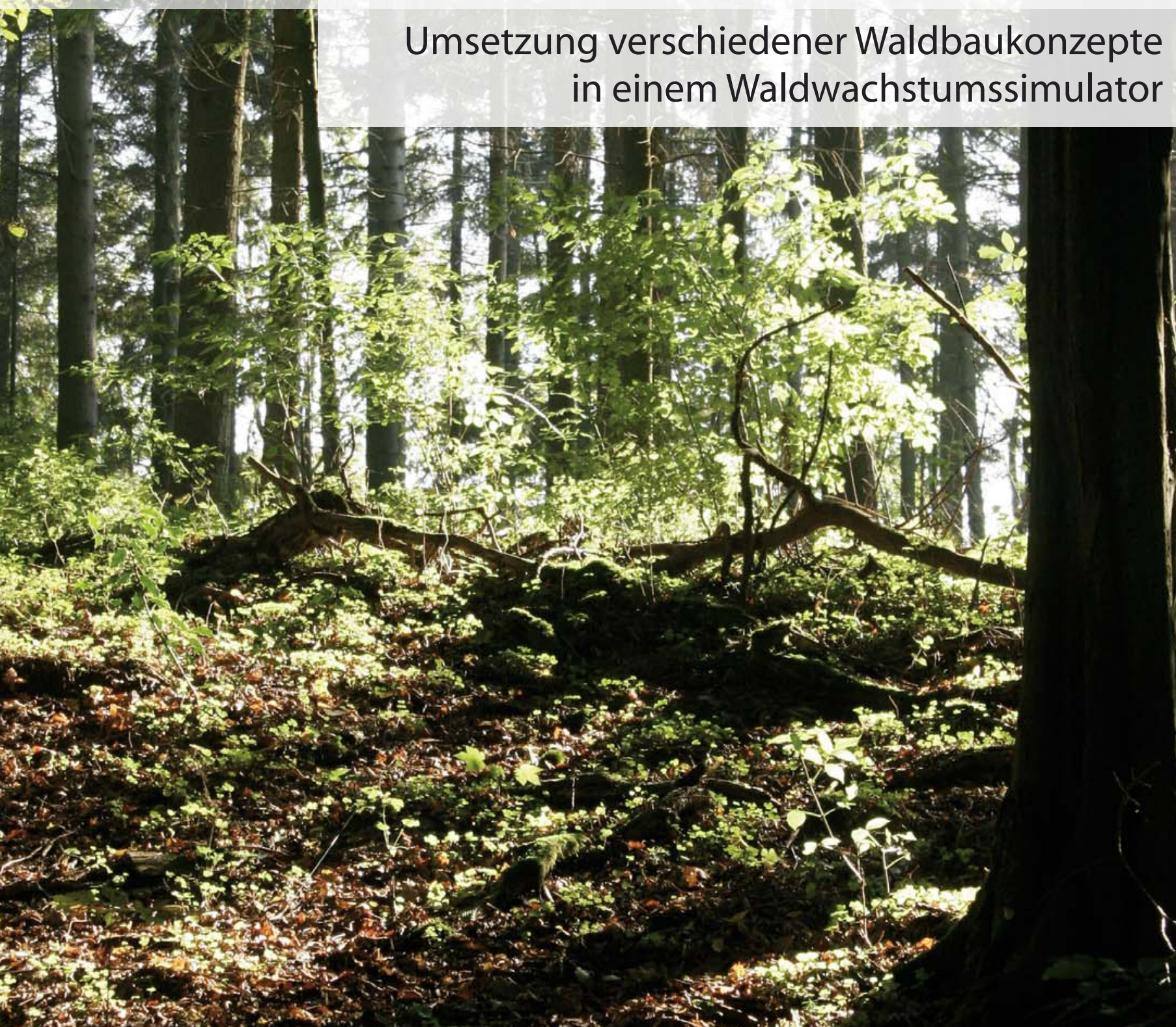
in einem Waldwachstumssimulator

Umsetzung verschiedener Waldbaukonzepte

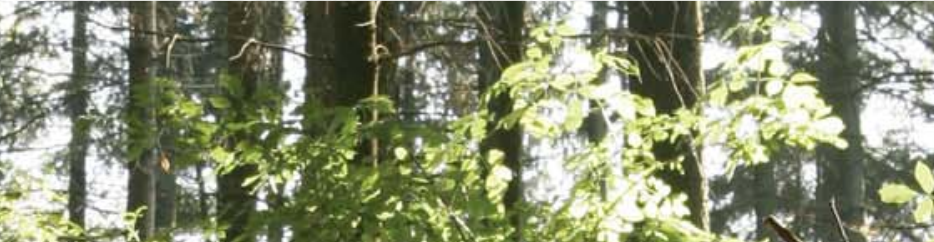
whon

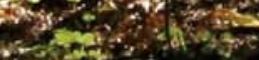

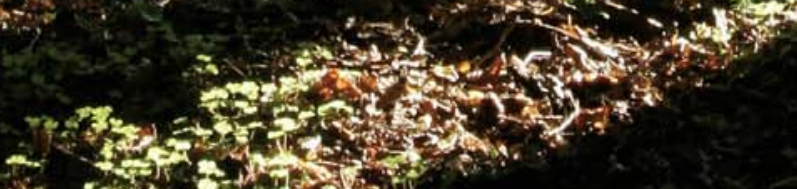



Duda $\cdot$ Vergleich forstlicher Managementstrategien 



\title{
Vergleich forstlicher Managementstrategien
}

\author{
Umsetzung verschiedener Waldbaukonzepte in einem \\ Waldwachstumssimulator
}

\begin{abstract}
Dissertation
zur Erlangung eines Doktorgrades

an der Fakultät für Forstwissenschaften und Waldökologie der Georg-August-Universität Göttingen
\end{abstract}

vorgelegt von Henriette Abigail Aline Duda, geboren in Köln 
(C) 2006 Henriette Abigail Aline Duda

Bild und Gestaltung des Covers: Simon Hilke, Simiridium

Herstellung und Verlag: Books on Demand $\mathrm{GmbH}$, Norderstedt

ISBN-10: 3-8334-6618-9

ISBN-13: 978-3-8334-6618-2

D7

Die vorliegende Dissertation wurde in elektronischer Form veröffentlicht.

Sie ist unter folgender Internetadresse zugänglich:

http://resolver.sub.uni-goettingen.de/purl/?webdoc-1300

1. Berichterstatter: Prof. Dr. Jürgen Nagel

2. Berichterstatter: Prof. Dr. Klaus von Gadow

Tag der mündlichen Prüfung: 25.08.2006 

„Es ist dafür gesorgt, daß die Bäume nicht in den Himmel wachsen." Johann Wolfgang von Goethe 


\section{Danksagung}

Schon Goethe wußte, daß allem eine natürliche Grenze gesetzt ist. Dennoch gab es Momente beim Verfassen dieser Arbeit, in denen ich an dieser Aussage zweifeln mochte. Gerade in diesen Situationen habe ich mich immer auf die fachliche und persönliche Unterstützung meines Umfelds verlassen können. Mein herzlicher Dank geht daher an alle, die es mir ermöglicht und erleichtert haben, die vorliegende Arbeit abzuschließen.

Die Förderung der Drittmittelprojekte „FOREST“1 und „Entwicklung eines Decision Support Systems für die betriebliche und regionale Waldbauplanung - DSSW“2 ermöglichten es mir finanziell, mich über drei Jahre mit meinem Thema zu beschäftigen und den vorliegenden Forschungsbeitrag leisten zu können.

Dem Leiter der Abteilung für Betriebswirtschaftslehre des Instituts für Forstökonomie der Universität Göttingen Prof. Dr. Bernhard Möhring danke ich für das Bereitstellen der ökonomischen Funktionen, die meine Arbeit bereichert haben.

Allen voran danke ich aber der motivierenden Betreuung durch meinen Doktorvater Prof. Dr. Jürgen Nagel, der die Forschung für mich zu einer spannenden und angenehmen Tätigkeit machte. Er stand mir mit konstruktiver Kritik zur Seite und half mir durch seine pragmatische Sichtweise, die Arbeit in einem angemessenen zeitlichen Rahmen abzuschließen. Inm und Herrn Klaus von Gadow danke ich dafür, daß sie sich als Gutachter für diese Arbeit zur Verfügung gestellt haben.

Die Nordwestdeutsche Forstliche Versuchsanstalt in Göttingen bot mir während des gesamten Arbeitszeitraums hervorragende Forschungsbedingungen. Mein Dank gilt hier besonders den Mitarbeitern der Abteilung Waldwachstum: Nahezu jeder Kollege hat auf seine Weise zum Gelingen dieser Arbeit beigetragen, sei es durch technische Unterstützung (Dank an Jochen Eggemann), durch die Erleichterung der Literaturrecherche (Dank an Brigitte Häseker) oder durch menschlichen Rückhalt von Kollegen. Bei fachlichen Fragen konnte ich stets kompetenten Rat vom jeweiligen Experten einholen. Meinen Bürokollegen Swen Hentschel und Jan Hansen danke ich für viele fruchtbare Diskussionen, die meinen Blickwinkel veränderten und meinen Horizont erweiterten. Das gute Arbeitsklima in der Nordwestdeutschen Forstlichen Versuchsanstalt ist nicht zuletzt ihrem Leiter Prof. Dr. Hermann Spellmann zu verdanken. Er prägt die hilfsbereite und fröhliche Atmosphäre des Arbeitskollegiums durch seine Wertvorstellungen und seine Persönlichkeit in entscheidendem Maße mit. Trotz arbeitsintensiver Zeiten hatte er regelmäßig ein offenes Ohr für mich.

Ich danke Peter Lins, Hardy Scheffczyk, Carmen Neikes, Hubertus Mascher und Simon Hilke, die an der Korrektur und dem Layout der Dissertation mitgearbeitet haben. Außerdem möchte ich mich für die gemeinsame Zeit mit meiner WG bedanken, die in unserer Küche Freud und Leid an zahllosen Abenden mit mir teilten. Seelischen Beistand erfuhr ich auch oft durch Jan Lachenit, meine Schwester Christiane Duda, Andrea Gierschner und Christin Leistner. Meiner Mutter bin ich dankbar für ihre Unterstützung, ihre Kraft und ihre Liebe, mit der sie mich fortwährend durch mein Leben begleitet.

1 Das Projekt wurde gefördert durch das BMBF unter dem Förderkennzeichen 01 LM 0207.

2 Das Projekt wurde gefördert durch die DBU unter dem Aktenzeichen 19872. 


\section{Inhaltsverzeichnis}

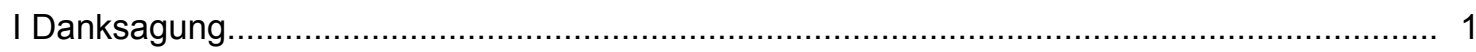

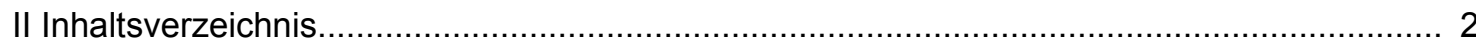

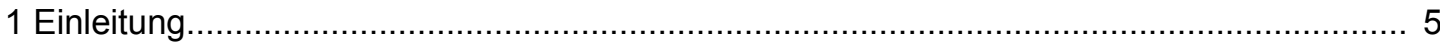

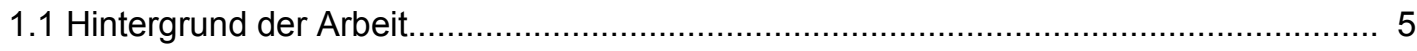

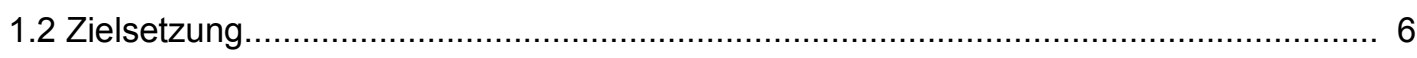

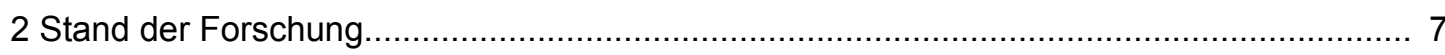

2.1 Begriffsdefinition Managementstrategie..................................................................... 7

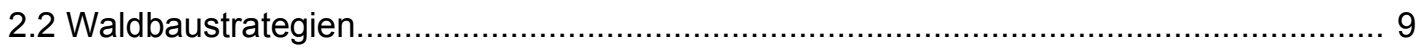

2.2.1 Besonderheiten von Strategien im Waldbau..................................................... 9

2.2.2 Waldbaustrategien in Deutschland................................................................... 12

2.2.3 Umsetzung von Waldbaustrategien durch die Anwendung verschiedener

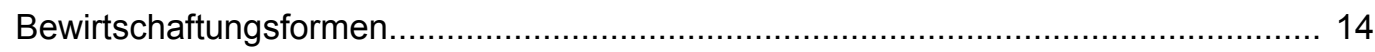

2.3 Die Eignung planungsunterstützender Instrumente ........................................... 22

2.3.1 Betrachtung verschiedener Waldwachstumsmodelle.......................................... 23

2.3.2 Einzelbaumwachstumssimulatoren als geeignete Instrumente zum Vergleich

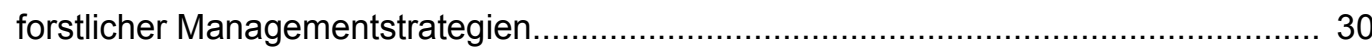

2.4 Abbildung verschiedener Bewirtschaftungsformen in Einzelbaumwachstumsmodellen 33

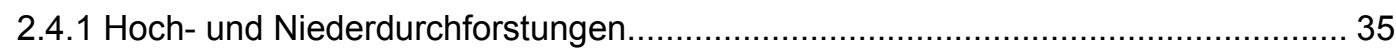

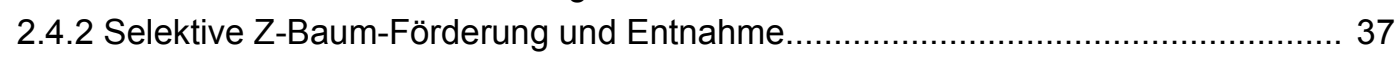

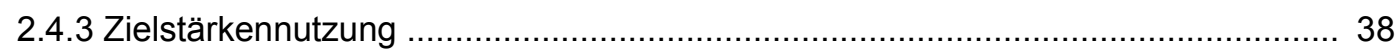

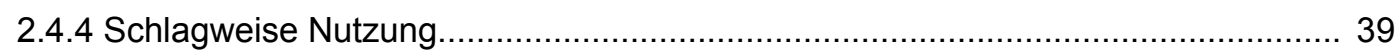

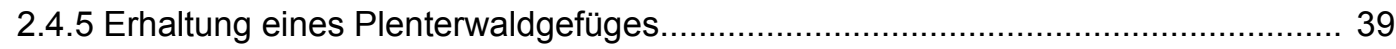

2.4.6 Sonstige Ansätze zur Abbildung forstlicher Eingriffe............................................. 39

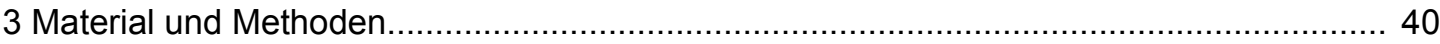

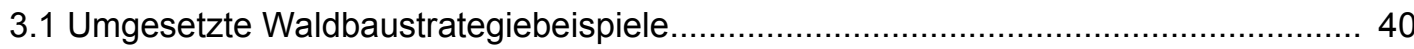

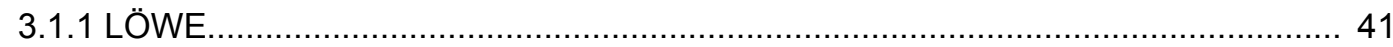

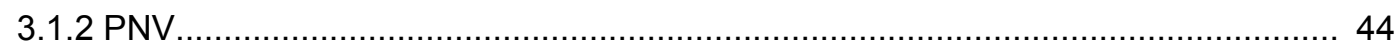

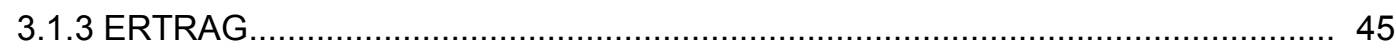

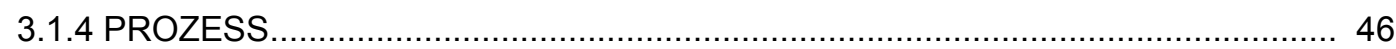

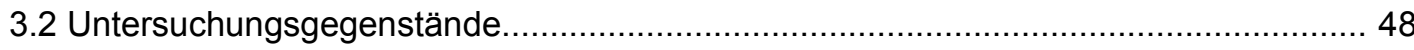

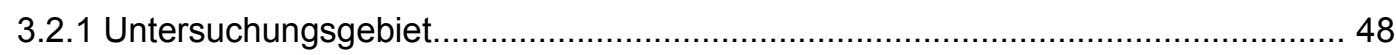

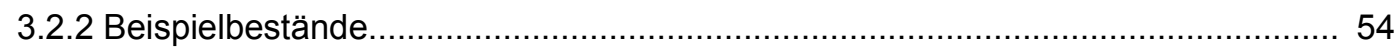

3.3 Überblick über Simulation und Auswertung.................................................... 56

3.3.1 Auswahl und Weiterentwicklung eines geeigneten Simulationsinstruments............56

3.3.2 Verarbeitungsschritte und Datenhaltung....................................................... 57

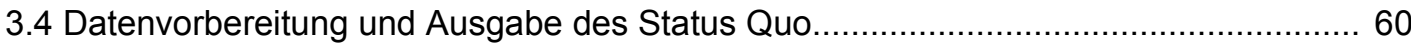

3.4.1 Einlesen der Forsteinrichtungsdaten und Generierung der Simulationsbestände... 60

3.4.2 Bestimmung der Zieltypen und Berechnung der Auswertungsparameter................64

3.5 Szenarienabhängige Fortschreibung und Ausgabe der Simulationsergebnisse...........67

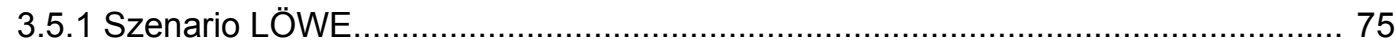




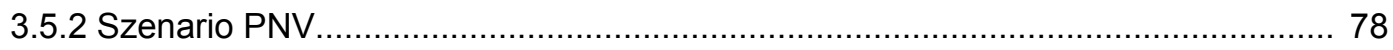

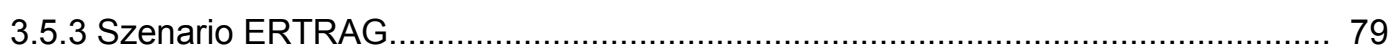

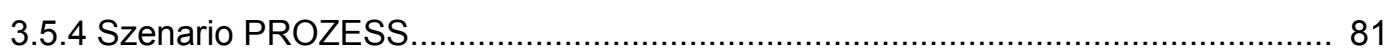

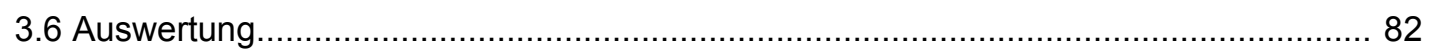

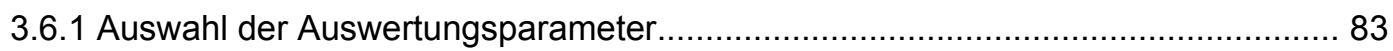

3.6.2 Herleitung der Auswertungsparameter............................................................. 86

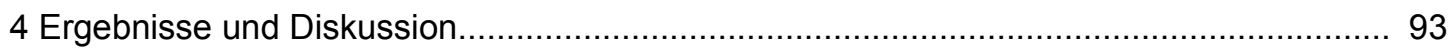

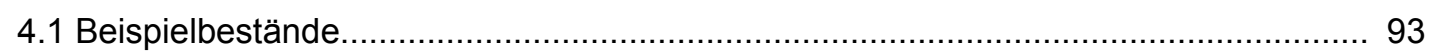

4.1.1 Beispiele für die Anwendung der Durchforstungsalgorithmen............................. 93

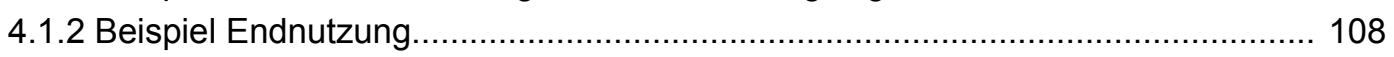

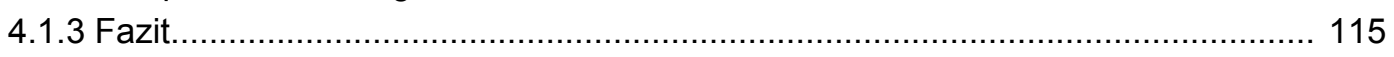

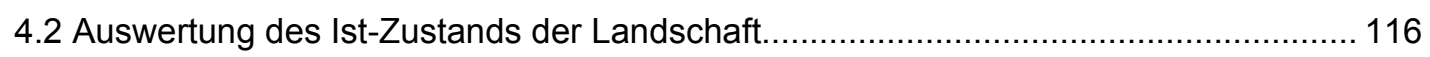

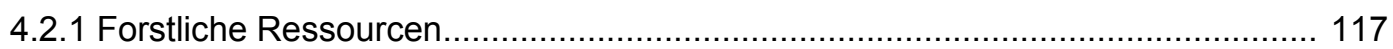

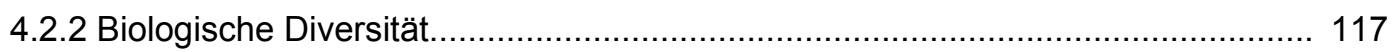

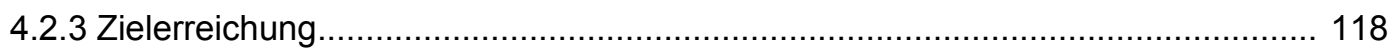

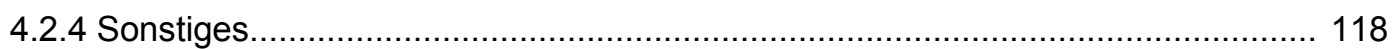

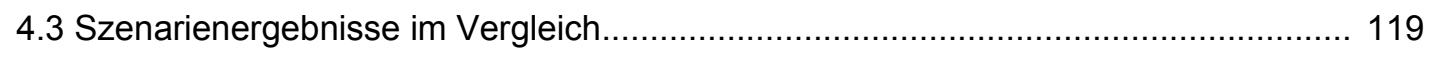

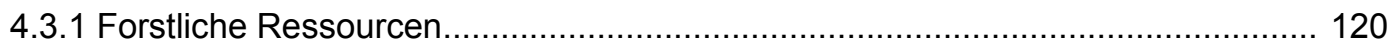

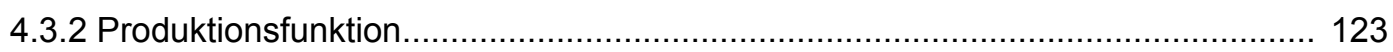

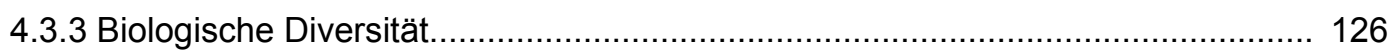

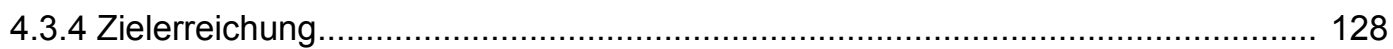

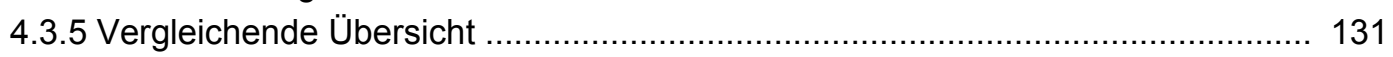

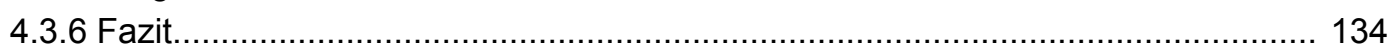

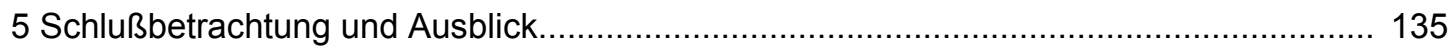

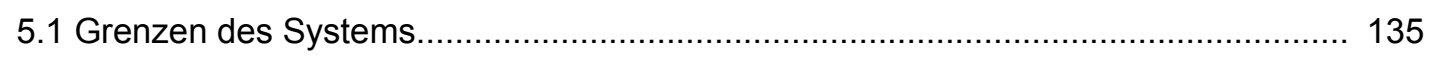

5.2 Weiterer Forschungsbedarf, Verbesserungen...................................................... 136

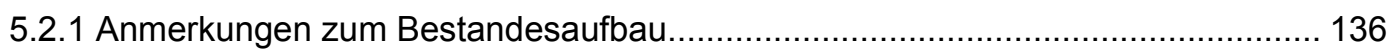

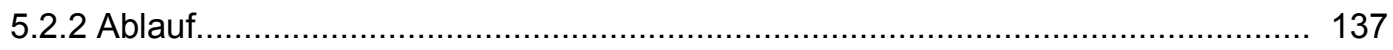

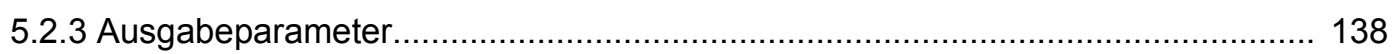

5.3 Anwendungsgebiete und Implementierungsmöglichkeiten...................................... 140

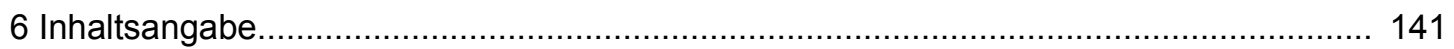

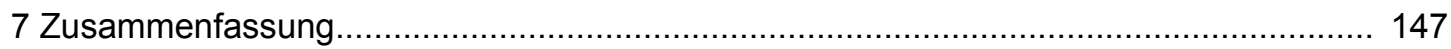

7.1 Vergleich forstlicher Managementstrategien - Umsetzung verschiedener Waldbaukonzepte in einem Waldwachstums-simulator.

7.2 Comparison of Forest Management Strategies - Realization of Silviculture Concepts within a Forest Growth Simulator..................................................................... 148

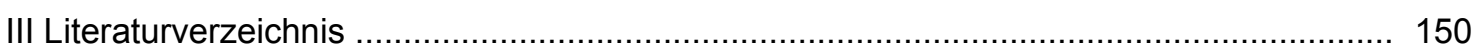

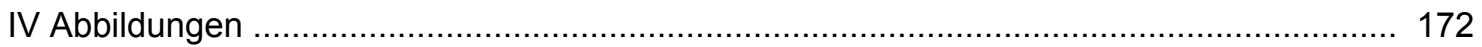

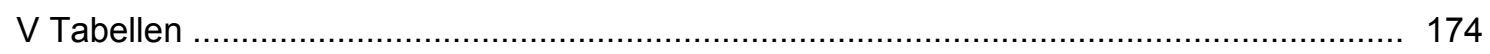

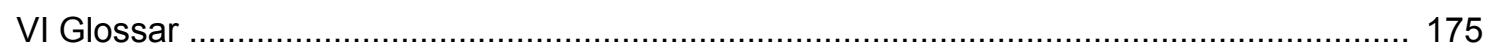

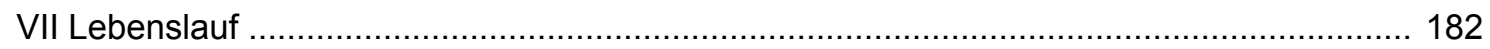


„Weißt Du, was ein Wald ist?

Ist ein Wald etwa nur zehntausend Klafter Holz?

Oder ist er eine grüne Menschenfreude?"

Bertolt Brecht 


\section{Einleitung}

\subsection{Hintergrund der Arbeit}

Im dem Zitat der vorigen Seite, das Brecht seinem trinkfreudigen Herrn Puntila in den Mund legte, klingt die Skepsis an einer rein wirtschaftlichen Funktion des Waldes an. Der Wald wurde in seiner Geschichte auch immer als allgemeines Kulturgut wahrgenommen. Literatur und Dichtung der Vergangenheit und Gegenwart spiegeln wider, welche Bedeutung dem Wald zugemessen wird. Da das Landschaftsbild Deutschlands zu etwa einem Drittel von Wald geprägt ist (BMVEL 2003) und verschiedene Interessengruppen eine eigene Interpretation von der „grünen Menschenfreude“ anmelden, werden Debatten über eine angemessene Bewirtschaftung des Waldes oft emotional und konfliktträchtig geführt. Für die Verantwortungsträger stellt sich daher die Frage nach sachlichen Entscheidungshilfen für eine zeitgemäße Waldbewirtschaftung, die den unterschiedlichen Interessen entgegenkommen und entsprechen soll. Damit sind vor allem die rund 2 Mio. Waldbesitzer gemeint, die in ca. 29000 Betrieben organisiert sind (MROSEK, KIES UND SCHULTE 2005). Viele dieser Betriebe verfolgen selbstgesteckte Ziele, die sie durch die Umsetzung unterschiedlicher Managementstrategien anstreben. Dazu kann zum Beispiel die Erwirtschaftung eines angemessenen Gewinns genauso gehören wie der Schutz gewisser Landschaftselemente. An die Entscheider der Betriebe werden von außen vielfältige Ansprüche und Forderungen herangetragen, die bei Bewirtschaftungsfragen berücksichtigt werden können:

Gesetzliche Bestimmungen oder am öffentlichen Interesse orientierte Maßnahmen lassen oft nicht $z u$, daß die Betriebe völlig autonom und unabhängig entscheiden. Die gesellschaftlichen Anforderungen an den Schutz und die Nutzung des Waldes werden oft von Interessenverbänden wie Tourismus- und Sportvereinen, Waldkindergärten oder Naturschutzorganisationen an den Waldbesitzer herangetragen. Es ist heute gesellschaftlicher Konsens, die Wälder als natürliche Ressource zu schützen und zu pflegen. Auch die volkswirtschaftliche und arbeitsmarktpolitische Bedeutung der deutschen Forstwirtschaft rückt derzeit wieder in den Vordergrund. Aus der „Clusterstudie Forst und Holz Deutschland 2005“ (Mrosek, KIES UND Schulte 2005) geht hervor, daß die Forstwirtschaft als wichtigste Rohstoffquelle der nachgelagerten Holzwirtschaft dient und somit direkt und indirekt als wichtiger Arbeitgeber fungiert. Übergreifend gibt es verschiedene Vorstellungen, wie Schutz, Pflege und Nutzung in Einklang gebracht werden können. Eine Vielfalt von Akteuren, hinter denen sich eine noch größere Zahl von Motiven und Argumenten verbirgt, versucht den politischen Prozeß zu beeinflussen. Immer häufiger fordern die Öffentlichkeit oder bestimmte Interessengruppen eine Beteiligung an der waldbaulichen Praxis (von TEUfFEL ET AL. 2006).

Um auf sachlicher Ebene Entscheidungen treffen zu können und ein in sich schlüssiges Bewirtschaftungskonzept zu erarbeiten, ist es wichtig, die langfristigen Auswirkungen von Waldbewirtschaftungsmaßnahmen prognostizieren zu können. Hat ein Betrieb aus der Menge an Anforderungen jene Managementziele herauskristallisiert, die er mit seinem Waldbehandlungskonzept umsetzen möchte, steht er vor der planerischen Herausforderung, seinen Wald so zu bewirtschaften, daß diese Ziele auch erreicht 
werden. Er benötigt zunächst ein Instrument, mit dem die aktuelle Situation analysiert werden kann. Auf der Basis dieser Situationsanalyse können weiterführende Ziele formuliert und anschließend entsprechende Bewirtschaftungsmaßnahmen geplant und umgesetzt werden. Die Einflüsse, die man mit einem Managementkonzept auf Schlüsselparameter des Waldes nehmen kann, sind für die Betriebsführung jedoch nicht immer transparent, da der Wald ein komplexes Beziehungsgefüge darstellt.

In dieser Arbeit wird ein Instrument vorgestellt, das Waldbesitzern eine wertvolle Hilfe bei Entscheidungen sein kann.

\subsection{Zielsetzung}

Die Wahl einer Waldbaustrategie ist eine klassische Entscheidungssituation (siehe Abbildung 1.2.a), bei der der Wunsch, eine Zielsetzung zu verwirklichen (zum Beispiel Waldumbau zugunsten von Laubmischwäldern), als Problem identifiziert wurde. Das Entscheidungsfeld besteht aus nicht beeinflußbaren Rahmenbedingungen (Waldzustand, Klima, Baumartenzusammensetzung...) und den vorstellbaren Handlungsalternativen (alternative Bewirtschaftungsformen). Neben der Identifikation von Handlungsalternativen ist auch ihre Bewertung von Belang, um die Auswirkungen der im Rahmen einer Strategie angewendeten Maßnahmen überprüfen zu können. Um angestrebte Ziele erreichen zu können, müssen geeignete Maßnahmen ausgewählt und der Zeitpunkt ihrer Durchführung festgelegt werden. Dabei sind Entscheidungen immer von der zu erwartenden Entwicklung der Bestände abhängig. Möchte man zum Beispiel eine nachhaltige Vorratshaltung realisieren, ist dies nur bei Kenntnis der Vorratsentwicklung des betrachteten Bestandes und letztendlich der Vorratsentwicklung des Betriebs möglich.

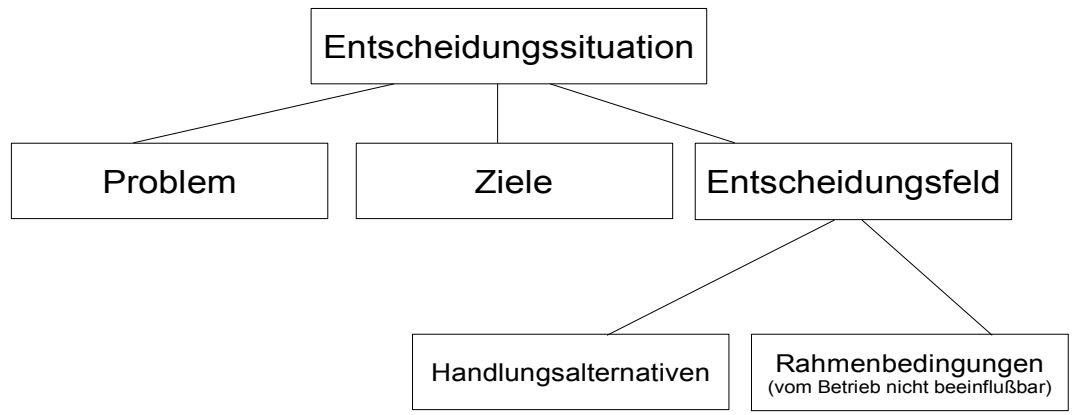

Abbildung 1.2.a: Elemente einer Entscheidungssituation (aus OEsten UND RoEDeR 2002).

Ziel dieser Arbeit ist es, die Entscheidung bei der Auswahl einer Waldbaustrategie zu erleichtern. Dazu werden verschiedene Managementstrategien in den Waldwachstumssimulator BWINPro (DöBbeLER ET AL. 2003, NAgel 2002) integriert, um die Auswirkungen von beispielhaft umgesetzten Strategien zu veranschaulichen. Um die nötige Transparenz für den Nutzer zu schaffen, werden folgende Anforderungen an die Simulation gestellt: 
Sie soll...

- auf einer Datengrundlage arbeiten, die in Forstbetrieben in der Regel vorhandenen ist.

- das Wachstum des Waldes unter Anwendung gängiger waldbaulicher Maßnahmen abbilden.

- $\quad$ sowohl bestandesbezogene als auch betriebsbezogene Kennzahlen ausgeben, da sich viele Managementziele nicht nur auf die Entwicklung einzelner Bestände, sondern auch auf die Entwicklung des Betriebs und des Landschaftsbildes auswirken.

- $\quad$ neben Volumina und Baumartenzusammensetzungen auch Strukturparameter und betriebswirtschaftliche Kennzahlen zur Verfügung stellen.

- die Ergebnisse in einer Datenbank ablegen, um einen flexiblen Datenzugriff zu ermöglichen.

- die Anbindung an ein Geographisches Informationssystem (GIS) bereitstellen.

- die Entwicklung eines Betriebs über einen mittel- bis langfristigen Zeitraum fortschreiben können, da sich vorgenommene Steuerungsmaßnahmen oft erst nach einigen Jahrzehnten im Bestandes- oder Landschaftsbild bemerkbar machen.

- für die weiteren Anpassungen flexibel sein.

Daher sollen die Steuergrößen getrennt von der Wahl der Maßnahmen angepaßt werden können. Außerdem ermöglicht ein komponentenweiser Aufbau des Programms die modulare Ergänzung von Maßnahmenelementen in der Zukunft.

\section{Stand der Forschung}

Dieses Kapitel gibt einen Überblick über die Bedeutung von Management und Strategie im allgemeinen (siehe Kapitel 2.1) und geht insbesondere auf die Rahmenbedingungen, die Entwicklung und die Umsetzung von Waldbaustrategien als Teile forstlicher Managementstrategien ein (siehe Kapitel 2.2). Es werden planungsunterstützende Instrumente des Waldbaus vorgestellt und die Möglichkeiten erörtert, diese zum Vergleich von Waldbaustrategien einzusetzen (siehe Kapitel 2.3 und 2.4). Dem Leser werden somit Hintergrundinformationen an die Hand gegeben, um die Auswahl der in dieser Arbeit eingesetzten Modelle und deren Weiterentwicklung nachvollziehen zu können.

\subsection{Begriffsdefinition Managementstrategie}

Der Begriff Managementstrategie läßt sich in die Komponenten Management und Strategie teilen. Das Wort Management stammt aus dem Lateinischen (manum agere = an der Hand führen) und ist in der Zusammensetzung „Managementstrategie“ in dem Sinn 
„Leitung (eines Betriebs) auf betriebswirtschaftlicher Grundlage“ gebraucht (WIKIPEDIA 2006A).

Wesentliche Definitionsmerkmale für (forstliches) Management sind nach OESTEN UND ROEDER 2002:

- die Zielorientierung

- die Gestaltung des (Forst-) Betriebs, d.h. die Konzipierung (der gedankliche Entwurf) des Forstbetriebs als handlungsfähige Einheit

- die Steuerung, d.h. die Bestimmung von Zielen sowie das Festlegen, Auslösen und Kontrollieren von zielgerichteten Aktivitäten in den übrigen Teilsystemen

- die Entwicklung des (Forst-) Betriebs - das ist das Ergebnis von Gestaltung und Steuerung des Betriebs im Zeitablauf

- die Berücksichtigung der Rahmenbedingungen, wie sie durch das Umsystem vorgegeben sind

Strategie besteht aus den griechischen Worten stratos (das Heer) und agein (führen). Der Begriff wurde lange im militärischen Sinn verwendet. So definiert der Kriegshistoriker von Clausewitz 1832 Strategie als "die Lehre vom Gebrauch der Gefechte zum Zweck des Krieges" (nachzulesen in VON ClausewITZ 1991). Im Licht des japanischen Sprichworts „Business is war" erscheint es konsequent, daß der Begriff seit ca. 1950 Einzug in die Betriebswirtschaftslehre gefunden hat (WIKIPEDIA 2006B). Im engeren Sinn ist die Unternehmensstrategie „ein genauer, langfristiger Plan des Vorgehens, um die definierten Ziele eines Unternehmens, die sogenannten Unternehmensziele, zu erreichen." (WIKIPEDIA 2006c). Als langfristige Planungsgrundlage (mit einem Zeithorizont von mehr als 4 Jahren) dient sie dem Unternehmen dazu, mittelfristige Ziele (in üblicherweise 2-4 Jahren) und kurzfristige Ziele (in weniger als 2 Jahren) zu erreichen. Im Laufe der Begriffsentwicklung rückt die langfristige Planung als wesentliches Strategieelement immer mehr in den Hintergrund. Für Porter ist zum Beispiel lediglich die Fähigkeit interessant, seinen längerfristigen Wettbewerbsvorteil zu sichern (PORTER 2003), und Henry Mintzberg hat die Definition der Strategie erweitert (Mintzberg, Ahlstrand und Lampel 2002), indem er sie anhand von fünf Punkten (den fünf „p“-s) aufhängt. Für inn kann Strategie folgendes sein:

- a plan (ein Plan - eine geplante Strategie als Weg oder/ und Zielbeschreibung)

- a pattern (ein Muster - ein Entscheidungsmuster, eine realisierte Strategie)

- a position (eine Position - die Positionierung im Markt)

- a perspective (eine Perspektive - die Verortung in der Umwelt, Wahrnehmung der Umwelt)

- a ploy (eine List - ein Manöver im Wettkampf)

Die Historie des Strategischen Managements geht auf die jahresbezogene Finanzplanung zurück (Jahresbudget), entwickelte sich dann über die langfristige Finanzplanung (Mehrjahresbudget, Abweichungsanalyse, Festlegung des Mittelbedarfs) zur Strategischen 
Planung als interaktivem Prozeß (im Sinne der Wertsteigerung des Unternehmens und der Sicherung von Wettbewerbsvorteilen) und von dort aus zum Strategischen Management (siehe Welge und Al-Laham 2003). Das Strategische Management beschäftigt sich unter anderem mit Unternehmensvision, -kultur und abgestimmten Managementverfahren. Bei der Entwicklung und der anschließenden Umsetzung einer Unternehmensstrategie findet in einem Managementprozeß der Abgleich des Unternehmens mit seiner Umwelt statt (Pfau 2001). Dieser Prozeß verlangt...

- $\quad$ strategische Zielplanung

- strategische Analyse

- Strategieentwicklung

- und Strategieimplemtierung

Die Reihenfolge der Elemente des Strategischen Managementprozesses ist nicht strikt festgelegt. Man kann immer wieder zu verschiedenen Teilen zurückkehren, wenn dies notwendig ist. So entsteht das Ergebnis als iterativer Prozeß.

In der strategischen Zielplanung werden Unternehmenspolitik und -leitbild sowie (vorläufige) strategische Ziele formuliert. In der Analysephase werden eine Umwelt- sowie eine Unternehmensanalyse durchgeführt, die eine Prognose zur zukünftigen Entwicklung ermöglichen. Je nach Analyseergebnis können die gesteckten Ziele modifiziert werden. Bei der eigentlichen Strategieformulierung unterscheidet man die Unternehmensstrategie, Geschäftsbereichsstrategien und funktionale Strategien, wie zum Beispiel Personalstrategie, Waldbaustrategie oder Marketingstrategie. Nach der Bewertung und Auswahl einer geeignet erscheinenden Strategie geht man in die Phase der Strategieimplementierung, die geplant, realisiert und kontrolliert wird.

Diese Arbeit bezieht sich auf den Vergleich von Waldbaustrategien, die als funktionale Strategien einen wesentlichen Teil der gesamtbetrieblichen Strategien von Forstbetrieben ausmachen und durch eine zielorientierte Gestaltung, Steuerung und Entwicklung des Forstbetriebs (Definition „Management“ nach OESTEN UND RoEDER 2002) ermöglicht werden.

\subsection{Waldbaustrategien}

\subsubsection{Besonderheiten von Strategien im Waldbau}

Da sich die Forstwirtschaft in einigen Merkmalen deutlich von anderen Wirtschaftszweigen unterscheidet, muß auch die Definition von einer forstlichen Managementstrategie an diese Gegebenheiten angepaßt werden. Speidel nennt sieben Eigenarten für Forstbetriebe (SPEIDEL 1984):

\section{Die Produktionsdauer}

Die Produktionszeit von Holz liegt deutlich über den Produktionszeiten, die für industriell hergestellte Waren gelten. Selbst schnellwachsende Baumarten, die zur 
Biomassenproduktion genutzt werden (wie die Pappel oder die Weide), haben in als Niederwald genutzten Kurzumtriebsplantagen Umtriebszeiten von zwei bis fünf Jahren (RIEDERICH 1999). Die Wertholzproduktion von Traubeneiche nimmt sogar einen Produktionszeitraum von ungefähr 240 Jahren ein, (NIEDERSÄCHSISCHE LANDESFORSTVERWALTUng 1989). Die Anforderungen an Produkte aus dem Wald ändern sich ständig und sind zu Beginn der Produktionszeit oft noch unbekannt. In Europa wurden zum Beispiel zu Anfang des 19. Jahrhunderts noch zahlreiche Eichen gepflanzt, da diese Baumart ein beliebtes Holz zur Schiffsherstellung lieferte. Gegen Ende des Jahrhunderts Iöste Eisen das natürliche Material zunehmend ab. Zum Zeitpunkt der Hiebsreife werden diese Bäume nun vollkommen anderen Verwendungen zugeführt.

Die langen Produktionszeiten stellen an die Gültigkeit von Waldbaustrategien die Anforderung, daß sie über Generationen hinweg verfolgt werden müssen, um langfristige Wettbewerbsvorteile sichern zu können. Unter einem mittelfristigen Planungshorizont werden in der Forstwirtschaft in jedem Fall längere Zeitabschnitte verstanden, als sie in anderen Teilen der Wirtschaft üblich sind. In der Regel gilt eine auf die nächsten zehn Jahre bezogene Planung als mittelfristig. Hinzu kommt, daß die Breite der Zeiträume groß ist, die zur Erreichung von Zielen gesteckt werden können. So können einige Ziele, die von der Produktionszeit unabhängig sind (zum Beispiel kundenorientierte Holzbereitstellung) relativ kurzfristig angestrebt werden, andere Ziele (wie eine bestimmte Holzqualität bei einer gegebenen Dimension) sind erst in einem generationenübergreifenden Zeitraum zu verwirklichen.

\section{Die Abhängigkeit von den natürlichen Bedingungen}

Boden-, Gelände- und Klimabedingungen sowie die Baumartenverteilung sind Fixwerte, auf die durch den Unternehmer mit technischen und wirtschaftlichen Mitteln nur sehr geringer oder gar kein Einfluß genommen werden kann. Waldbaustrategien haben daher nur einen begrenzten Spielraum. Die Änderung von langfristigen Zielen (wie die Änderung der Baumartenzusammensetzung) ist mit erheblichen organisatorischen und ökonomischen Veränderungen verbunden und sollte stets sorgfältig geprüft werden. Waldumbau ist in diesem Zusammenhang als strategische Neuausrichtung zu verstehen (siehe auch HANEWINKEL 1996).

\section{Die Schwierigkeit der Ertragsbestimmung}

Das Produktionsmittel Baum ist auch gleichzeitig Produkt, und die Produktreife für verwertbares Holz ist nicht eindeutig definiert, da ein Baum in nahezu jedem Alter genutzt werden kann. Diese Besonderheit macht die Überprüfung, ob eine Strategie zielführend ist, besonders schwierig.

\section{Die Produktion von forstlichen Dienstleistungen und die Bereitstellung einer forstlichen Infrastruktur}

Unter diesen Punkt fällt auch die zunehmende Bedeutung der Multifunktionalität der Wälder (siehe zum Beispiel Spellmann, Hillebrand und Cornelius 2001, Spellmann et al. 2004 
oder WAGNER 2004). Bei der Entwicklung einer Waldbaustrategie muß eine große ökologische und soziale Verantwortung im Umgang mit dem Wald wahrgenommen werden. Viele Produkte und Leistungen aus dem Wald sind nicht oder nur schwer substituierbar (zum Beispiel Luftfilterwirkung, Bereitstellung eines einzigartigen Lebensraums, Angebot eines Erholungsraums...). Vor diesem Hintergrund hängt eine Waldbaustrategie mehr als Strategien anderer Wirtschaftszweige von gesellschaftlichen Aspekten und öffentlicher Wahrnehmung ab.

\section{Die Bewertung einzelner Bestände und des gesamten Forstbetriebs}

Wirtschaftlichkeitsurteile haben nur Zwischenurteilscharakter, da ihre Kalkulation von einigen unbekannten (weil zukünftig festgelegten) Faktoren abhängt. So sind zum Beispiel Geldwertänderungen oder Verfahrensänderungen für die langfristige Bewertung essentiell, aber nicht $100 \%$ ig vorhersagbar. Es stellt sich somit auch immer die Frage, auf welcher Grundlage Entscheidungen über Maßnahmen getroffen werden können, mit denen gewisse Strategien verfolgt werden sollen. Zum Beispiel ist beim Umbau von Reinbeständen in ungleichaltrige Mischbestände zwar mit einer Minderung des Risikos von Kalamitäten und damit auch mit geringerem Ertragsausfall zu rechnen, allerdings ist der Waldumbau nicht kostenneutral umzusetzen. Beim Umbau von gleichaltrigen Fichtenbeständen zu ungleichaltrigen Mischbeständen sinkt zum Beispiel langfristig der Ertrag (Jacobsen, MöHRINg und WiPPeRmann 2004).

\section{Der Kapitalumschlag und der hohe Eigenkapitalanteil}

Der Kapitalumschlag (Definition siehe Glossar) ist mit 30 bis 80 Jahren im Vergleich zu anderen Wirtschaftszweigen besonders lang. Außerdem ist zu bedenken, daß Kapital (das größtenteils aus Eigenkapital besteht) langfristig im Produktionsmittel Baum, das gleichzeitig Produkt ist, gebunden wird. Auf Fremdkapital wird selten zurückgegriffen, da die durchschnittliche Rentabilität des Eigenkapitals geringer als die Verzinsung von Fremdkapital ist. Kalamitäten und eine Veränderung des Marktes können auf die aktuelle Nutzung, wie auf die langfristige Nutzungsplanung wesentlichen Einfluß nehmen. Daher ist eine gut kalkulierte Risikoverteilung essentiell.

\section{Die große Flächenausdehnung, Standortsgebundenheit}

Die große Flächenausdehnung führt dazu, daß Organisations- und Kommunikationsprobleme auftreten können. Eine geeignete Unternehmensstrategie bezieht diese Besonderheit mit ein. Zudem sind Forstbetriebe örtlich stark gebunden, Expansionsstrategien sind immer mit der Pacht oder dem Ankauf von Grundbesitz verbunden.

Eine Unternehmensstrategie, die langfristigen, richtungsweisenden Charakter haben soll, muß demnach die bedingte Anpassungsfähigkeit an veränderte Rahmenbedingungen berücksichtigen und über Generationen hinweg verfolgt werden, um langfristige Wettbewerbsvorteile sichern zu können. Dabei bleibt es nicht aus, daß sich eine Unternehmensvision und Unternehmenskultur entwickelt, die tradiert und modifiziert wird. 
Hier paßt die Vorstellung einer aus der Unternehmung emergenten (MinTZBERG, AhLSTRAND UND LAMPEL 2002) oder formierten (von selbst entstandenen) Unternehmensstrategie (siehe $\mathrm{KIRSCH}_{\mathrm{I}}$ 1997) mit stark evolutionärem Charakter gut zu der gängigen Entstehung von forstlichen Strategien.

\subsubsection{Waldbaustrategien in Deutschland}

Mit den wachsenden Anforderungen an die Waldwirtschaft entwickelten sich aus eindimensionalen Zieldefinitionen (zum Beispiel Nachhaltigkeit der Holzerzeugung (siehe HEYER 1841) oder Nachhaltigkeit der Geldbeträge (siehe Ostwald 1931)) mehrdimensionale Zielvorstellungen, die die Multifunktionalität der Wälder berücksichtigen (siehe auch Speidel 1972, Spellmann, Hillebrand und Cornelius 2001, Spellmann et al. 2004 oder WAGNER 2004).

Zu dieser Entwicklung hat auch die international geführte Diskussion (siehe auch KRONAUER 1992) über den Nachhaltigkeitsbegriff beigetragen, die mit dem Brundtland-Bericht (WORLD Commission of Environment and Development 1987) in die öffentliche Diskussion Einzug hielt. 1992 folgte daraufhin die Agenda 21, die als Ergebnis der Rio-Konferenz (UNITED Nations Conference on Environment and Development 1992) bis heute Grundlage für Diskussionen über den Begriff der Nachhaltigkeit auf EU-Ebene ist. Aufbauend auf die waldbezogenen Kapitel der Agenda 21 entwickelte die Ministerkonferenz in Helsinki (Ministerial Conference 1993) ein Konzept zur nachhaltigen Waldbewirtschaftung in Europa. 1998 wurde ein gesamteuropäischer Katalog von Kriterien, Indikatoren und Leitlinien einer nachhaltigen Waldbewirtschaftung verabschiedet (nachzulesen in LoISKEKOSKI UND HALKo 2000), der auch in Deutschland in die Forstpolitik einfließt (siehe zum Beispiel ScHWORER 2001, BMVEL 2003).

Neben der internationalen Nachhaltigkeitsdiskussion prägten nationale Entwicklungen die deutsche Forstwirtschaft. In den 70er Jahren wurde zum Beispiel ein Fokus auf Mechanisierung und Rationalisierung gelegt, um Pflegerückstände beseitigen zu können. In dieser Zeit wurden Landeswaldgesetze und 1975 auch das Bundeswaldgesetz erlassen. Die 80er Jahre waren von den Sorgen um neuartige Waldschäden, dem „Waldsterben“ geprägt (siehe zum Beispiel STERN ET AL.1979). 1989 wurden die Kriterien der ordnungsgemäßen Forstwirtschaft vom Agrarministerium des Bundes und der Länder auf der Grundlage der Vorschläge des Deutschen Forstwirtschaftsrats konkretisiert (KöPF 2002). Nach den großen Sturmschäden in der Zeit von Ende Januar bis Anfang März 1990 (durch die Orkane Vivian und Wiebke) stieg in den 90er Jahren das Bedürfnis nach naturnahem Waldbau, von dem eine Strukturverbesserung und Stabilisierung der Wälder erwartet wird (siehe zum Beispiel Spellmann 1996). Aus diesen Jahren stammen neben dem nationalen Forstprogramm für Deutschland (siehe BMELF 2000) auch viele Waldbauprogramme (auch Waldprogramme genannt) der Bundesländer ${ }^{3}$. In diesen Regierungsprogrammen sind die Waldbaustrategien der Länder formuliert. Sie enthalten häufig ein Leitbild zum naturnahen Waldbau und definieren globale Ziele und

3 Siehe zum Beispiel LFV NRW 1990, Niedersächsische Landesforstverwaltung 1991, Hessisches Ministerium für Landesentwicklung, Wohnen, LandWirtschaft, Forsten und Naturschutz 1993, Ministerium für ERnährung, Landwirtschaft, Forsten und Fischerel 1996 oder ThüringenForst 2006 A und THÜRINGENFORST 2006 B. 
Verhaltensmuster (oft "Grundsätze“ genannt) für die Umsetzung; teilweise verweisen sie auch auf Quellen, die konkrete Handlungsanweisungen enthalten. Waldbauprogramme sind in Abgrenzung zum Normativen und Operativen dem Strategischen Management zuzuordnen (siehe ULRICH 2001). Im folgenden wird von einem Waldbaukonzept als dem Teil einer Waldbaustrategie gesprochen, der unterhalb des Leitbilds als Sachgebietsstrategie den Rahmen für konkrete Ziele, Handlungsmuster und Maßnahmen (dem operativen Management) steckt. In der Abbildung 2.2.2.a ist die Einordnung eines Waldbauprogramms in den Zusammenhang von forstpolitischen Zielvorgaben, Betriebspolitik, strategischem und operativem Management am Beispiel der Landesforstverwaltung Rheinland-Pfalz dargestellt.

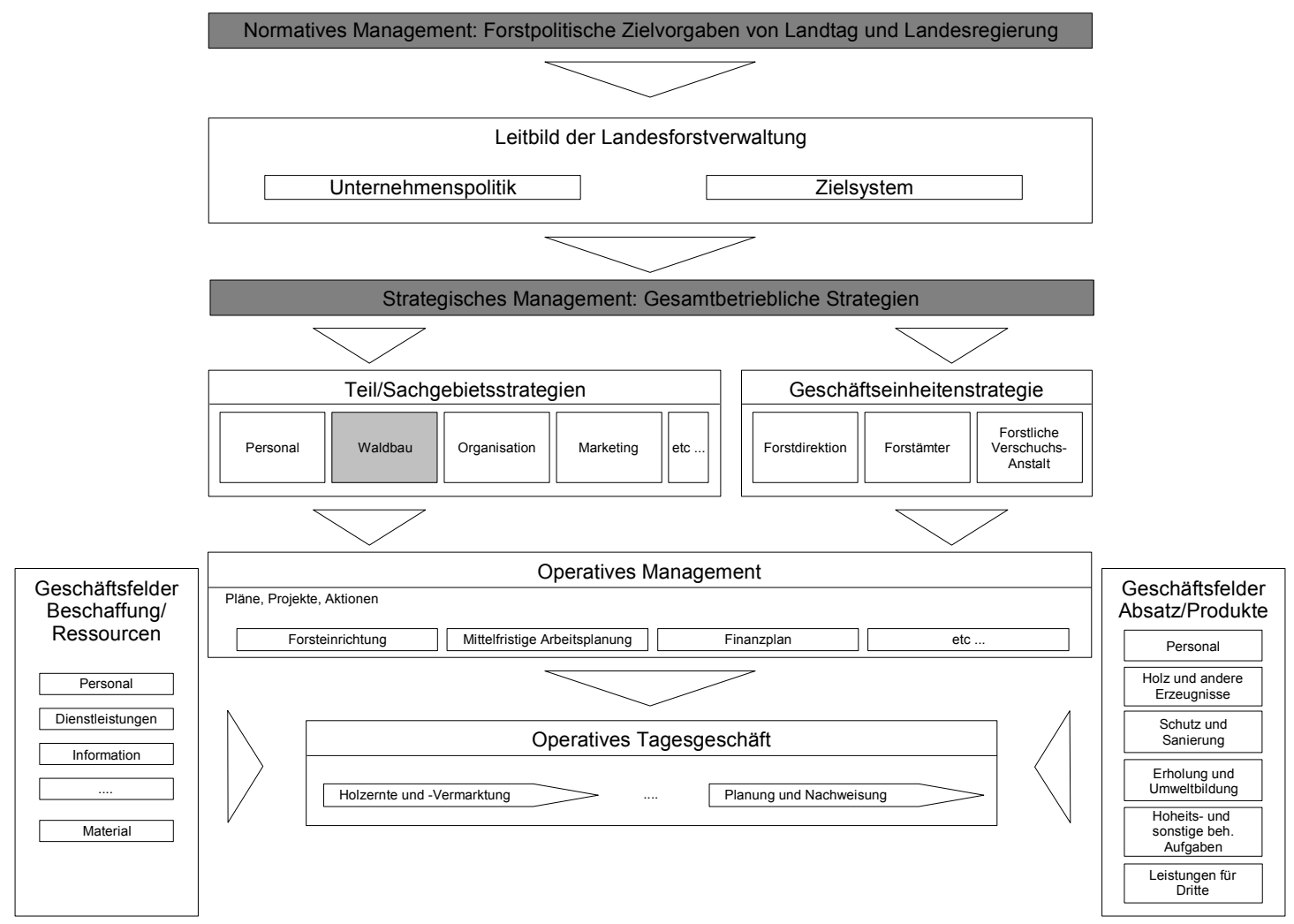

Abbildung 2.2.2.a: Einordnung eines Waldbaukonzepts im Zusammenhang von forstpolitischen Zielvorgaben, Betriebspolitik, strategischem und operativem Management am Beispiel der Landesforstverwaltung Rheinland-Pfalz (nach OESTEN UND RoEder 2002).

Eine Zertifizierungskampagne, die von Umweltverbänden Ende der 90er Jahre ins Leben gerufen wurde, nimmt derzeit Einfluß auf die Strategien der Forstbetriebe. In den Jahren 1994 und 1995 wurde im Stadtwald Lübeck ein über die genannten Landesprogramme hinausgehendes Konzept einer naturnahen Waldbewirtschaftung entwickelt, das auf dem Schutz natürlicher Prozesse im Wald basiert, (siehe „Prozeßschutzkonzept“ in Sturm 1993, Sturm 1994, StuRm 1995 und FÄSER 1995). In diesem Zusammenhang wurden Holzzertifizierungslabels (Naturland und FSC) von den Umweltschutzorganisationen GREENPEACE und BUND in Leben gerufen. Mittlerweile unterstützen Vertreter verschiedenster Waldbesitzarten, Sozialverbände und der Holzwirtschaft unter dem Label 
PEFC (siehe zum Beispiel (Gruss 2004) oder (TeegelbeKkers 2001)) die Produktion von zertifiziertem Holz.

Auf Waldbaustrategien haben nach dieser Entwicklung heute also sowohl der Waldzustand, örtliche Gegebenheiten, langjährige Erfahrungen, Kosten und Erlöse sowie politische Rahmenbedingungen und Zertifizierungskriterien Einfluß. Gegebenenfalls können wissenschaftliche Erkenntnisse zu einer Neuorientierung führen. Ein Beispiel für ein theoretisches Konzept, das seit seiner ersten Definition immer wieder in die Landschaftsplanung und besonders den Naturschutz einfließt, ist die von Tüxen 1956 definierte „Potentielle Natürliche Vegetation“ (pnV) (siehe TüxEN 1956).

Man kann davon ausgehen, daß private Waldbesitzer ebenso wie die Landesforstverwaltungen Strategien verfolgen, allerdings fehlen hier schriftliche Quellen. Dieser Umstand sollte jedoch nicht als Fehlen einer Strategie gedeutet werden, da laut Definition eine Unternehmensstrategie nicht der schriftlichen Form bedarf (siehe Mintzberg, Ahlstrand und Lampel 2002 und Kirsch 1997), sondern auch gewachsene und "gelebte" Strategien ihre Gültigkeit besitzen. Eine Studie unter 1500 Privatwaldbesitzern in Baden-Württemberg zeigte zum Beispiel, daß die derzeitige Tendenz zur naturnahen Waldwirtschaft auch auf das Wirtschaften im Privatwald Einfluß hat (siehe BIELING UND SCHRAML 2004).

\subsubsection{Umsetzung von Waldbaustrategien durch die Anwendung verschiedener Bewirtschaftungsformen}

Zur Umsetzung von Waldbaukonzepten kann man zwischen unterschiedlichen Bewirtschaftungsformen wählen. Je nach Zielsetzung des Betriebs (zum Beispiel Wirtschaftlichkeit, Nachhaltigkeit der Holzproduktion, Produktion von Massenware oder Qualitätsware, Arten- und Biotopschutz, Förderung von biologischer Vielfalt oder Prozeßschutz) kommen alternative Bewirtschaftungsformen in Betracht. Die Ausgangslage (Struktur) des Betriebs, die Marktsituation, die zur Verfügung stehende Technik und die künftig erwartete Entwicklung aller Komponenten haben Einfluß auf die Wahl von geeigneten Maßnahmen. Dabei unterscheidet man Maßnahmen zur Verjüngung und zur Jungwuchspflege, der Läuterung, der Wertästung, der Durchforstung sowie der Endnutzung.

Die Umsetzung eines waldbaulichen Ziels ist eng an die Wahl der Maßnahmen gekoppelt. Zum Beispiel ist die Produktion von Massenware in gleichaltrigen Reinbeständen geeignet umzusetzen, wohingegen die Zielstärkennutzung für die Wertholzproduktion besser geeignet ist. Die Wahl einer bestimmten Maßnahme (zum Beispiel Zielstärkennutzung) kann wiederum Auswirkungen auf die Wahl anderer Maßnahmen haben, da nur die Kombination der beiden Maßnahmen die Bestandesentwicklung in gewünschter Weise beeinflussen. Zum Beispiel kann man bei geplanter Zielstärkennutzung durch Einzelbaumförderungen bei der Durchforstung ausgewählte Individuen schnell zur Zielstärke führen und dabei ihre Qualität sichern. 
Die Wahl und die Kombination verschiedener Maßnahmen kann auch von generellen (selbst oder durch die Umwelt auferlegten) Einschränkungen abhängen. Solche sind zum Beispiel:

- weitgehender/ grundsätzlicher Kahlschlagsverzicht

- grundsätzliche Einzelstammnutzung

- $\quad$ unbedingte Bevorzugung von Naturverjüngung

- $\quad$ kein Großmaschineneinsatz

- $\quad$ begrenzte Erschließung

- $\quad$ keine Räumung

- keine Bodenbearbeitung

- Total- oder Teilverzicht auf den Einsatz von Chemikalien gegen Insekten, unerwünschte Pflanzen oder Pilze

- $\quad$ seltene/ frühe oder mehrfache Eingriffe

- Pflegeeingriffe weitgehend unterlassen

- Anstreben einer Vorratsvermehrung

- Anstreben einer bestimmten Arten- und/oder Alterszusammensetzung

Die Festlegung solcher Maximen in den Waldbauzielen schränkt den Kreis der möglichen Handlungsalternativen zusätzlich ein. Die folgende Beschreibung beschränkt sich auf die Bewirtschaftungssysteme der Betriebsart „Hochwald“. Man unterscheidet Formen, die dem schlagweisen Hochwald zugerechnet werden (zum Beispiel Kahlschläge, Schirmschläge, Femelschläge, Saumschläge oder kombinierte Verfahren) von Plenterverfahren und der Naturgemäßen Waldwirtschaft, die sich in der Regel der Zielstärkennutzung bedienen (Dengler 1992 в und Burschel und Huss 1997).

Das Waldbausystem „schlagweiser Hochwald“ stammt aus der Zeit der forstlichen Klassik (18. Jahrhundert und Anfang des 19. Jahrhunderts). Man teilt dabei den Wald in sogenannte „Schläge“ ein, die zu unterschiedlichen Zeitpunkten hiebsreif sind. Erfolgt die Ernte in Schlägen, so führt dies zur Entstehung eines einschichtigen Altersklassenwaldes. In Ausnahmefällen können auch im schlagweisen Hochwald zweischichtige Bestände entstehen (zum Beispiel durch starke Auflichtung eines Bestandes durch Sturm oder Schnee und anschließender Pflanzung); man spricht dann von zweihiebigen Hochwäldern (DENGLER 1992 в). Je nach Endnutzungszeiträumen wird eine Bepflanzung der Kahlflächen nötig, oder der Bestand kann sich auf den entstehenden Freiflächen selbst verjüngen.

Der Plenterwald arbeitet im Gegensatz zu den zuvor beschriebenen Vorgehensweisen des schlagweisen Hochwaldes nicht mit der räumlichen Trennung von Altersstufen (siehe auch ScHÜTz 2001). Die Bestände eines Plenterwaldes setzten sich aus Bäumen aller Alters- 
bzw. Durchmesserstufen zusammen. Die Stammzahl in den Alters- oder Durchmesserklassen nimmt mit zunehmendem Alter bzw. BHD ab. In diesem Plenterwaldgefüge wird die Anzahl der Bäume pro Durchmesserklasse als Fließgleichgewicht konstant gehalten. Besonders geeignet für den Plenterwald ist ein Mischwald mit schattentoleranten Arten aus Buche, Fichte und Tanne (MITSCHERLICH 1952). Lichtbedürftige Baumarten wie Eiche, Kiefer oder Lärche sind kaum in eine solche Struktur zu integrieren. Die kleinflächige Struktur hat zur Folge, daß nur wenige Stämme zur gleichen Zeit in relativ kurzen Intervallen entnommen werden und auch nur kleinräumige Verjüngungflächen entstehen. Stärkere Eingriffe gefährden den künstlichen Gleichgewichtszustand des Plentergefüges. Die Nutzung von Stämmen ist durch das zu erhaltende strukturelle Gefüge immer gleichzeitig als Verjüngungs- oder Erziehungsmaßnahme zu verstehen.

Die naturgemäße Waldwirtschaft hat in einigen Forstbetrieben eine lange Tradition. Ein klassisches Beispiel ist das Revier Bärenthoren (siehe zum Beispiel KRUTZSCH UND WECK 1934). Der Begriff wurde in besonderem Maße durch die Arbeitsgemeinschaft Naturgemäße Waldwirtschaft (ANW) (Gündungsaufruf siehe WoBst 1950) in die Waldbaudiskussion eingebracht. Sie definierte 1950 Ziele eines integralen Waldbaus (siehe KRUTZSCH 1950). Zu diesen Zielen gehören die Förderung von Mischbeständen, Strukturrreichtum, standortsgemäße Baumartenwahl, hohe Vorräte, Qualitätsholzproduktion und die Berücksichtigung ökonomischer, ökologischer und landeskultureller Belange. In der naturgemäßen Waldwirtschaft werden kahlschlagfreie Endnutzungsmethoden in einem Betrieb kombiniert. Das gesamte Nutzungskonzept geht im Gegensatz zum Plenterwald jedoch nicht soweit, daß es die Erhaltung der Plenterwaldstruktur impliziert. Es übernimmt Ansätze aus dem Prinzip des Dauerwaldes, das von Alfred Möller in die deutsche Waldbaudiskussion eingebracht wurde (siehe zum Beispiel SIEBER 1928, MöLler 1992). Besonderes Gewicht wird in der naturgemäßen Waldwirtschaft auf Zielstärkennutzung (REININGER 1987) gelegt, für die eine Differenzierung des Bestandes durch positive Baumauslese und Förderung unabdingbar ist (HANIEL UND KLEINSCHMIT 1992). Durch verlängerte Endnutzungs- und Verjüngungszeiträume entstehen oft zwei- oder mehrstufige Bestände, deren Durchmesserverteilung nicht statisch beibehalten werden muß. Die Form der Nutzung und Endnutzung ist auch von der angestrebten Verjüngung abhängig. In der Regel soll eine natürliche Verjüngung der Bestände ermöglicht werden. Bei der Verjüngung von Lichtbaumarten ist daher ein differenziertes Vorgehen gefordert. Möchte man zusätzliche Arten einbringen (um eine bestimmte Artenmischung zu realisieren), sind auch Pflanzungen vorgesehen und müssen durch entsprechende Endnutzungskonzepte ermöglicht werden. Schattentolerante Arten werden in Umbaubeständen, in denen die Artenzusammensetzung grundsätzlich geändert werden soll, oft auch als Unterbau eingebracht, der nach der Endnutzung den Folgebestand prägt. (Dengler 1992 в und Burschel und Huss 1997).

Die mit Waldbausystemen verknüpften Maßnahmen unterscheidet man in Eingriffe zur Endnutzung, Jungwuchspflege, Läuterung, Wertästung oder Durchforstung. Im folgenden sind diese Begriffe näher erläutert, um einen Überblick über die vielseitigen Steuerungsmittel zu geben. 


\section{Endnutzung}

Als Endnutzung werden die Nutzungen eines hiebsreifen Bestandes oder Einzelbaums bezeichnet. Als hiebsreif gilt ein Einzelbaum, wenn er die angestrebte Zielstärke erreicht hat. Ein Bestand geht in die Endnutzung über, wenn eine ausreichende Anzahl von Stämmen zielstark ist. Nach der Definition von Bonnemann (BoNNEMANN 1967) hat eine Endnutzung die Verjüngung des Bestandes zum Ziel oder macht sie erforderlich. Kramer erweiterte den Begriff um die Nutzung des Oberstands eines zweischichtigen Bestandes, den Aushieb von Überhältern als Restnutzung des vorhergehenden Bestandes, alle Nutzungen in plenterartigen Hochwäldern, alle Nutzungen in Mittelwaldbeständen (die der Hochwaldbetriebsklasse angeschlossen sind) und die Nutzung von Flächen, die langfristig der Holzerzeugung entzogen werden sollen (KRAMER 1976). Die einsetzbaren Endnutzungsvarianten hängen eng von der Bestandesbehandlung bis zur Hiebsreife ab und sind in der Regel mit typischen Verjüngungs- und Entwicklungsmöglichkeiten verknüpft.

In Deutschland werden in der Regel keine Riesen- oder Großkahlschläge (die über 50 bzw. über fünf Hektar groß sind), sondern nur Kahlschläge (über einem Hektar) und Kleinkahlschläge (unter einem Hektar) angewendet (Definition siehe BURSCHEL UND Huss 1997). Infolge von Kalamitäten oder bei Aufforstungsflächen können jedoch Flächen entstehen, die vom Charakter her Großkahlschlägen entsprechen. Klassische Kahlschläge sind mindestens einen Hektar groß und haben mindestens eine Breite, die dem Dreifachen einer Baumlänge aus dem Altbestand entspricht (Definition nach WiedemanN 1926). Sie werden in der Regel durch Pflanzung verjüngt. Der gleichaltrige Aufbau und der hohe Massenanfall bieten bei der Ernte technische Rationalisierungsmöglichkeiten. Als Kahlschlagsvariante ist der Streifenkahlhieb zu nennen, der sich durch seine größere Breite vom Saumschlag abgrenzt.

Als Saumschläge gelten Endnutzungsmaßnahmen, die in Streifen bis zur Länge eines Altbestandesbaums durchgeführt werden (WAGNER 1912). Durch die geringe Breite der Freifläche wird natürliche Verjüngung ermöglicht. Dabei findet man sowohl gute Bedingungen für Schattbaumarten (in der Nähe des Altholzschirms) als auch für Lichtbaumarten (auf der nicht überschirmten Fläche). Man unterscheidet die Verfahren anhand der Form und Richtung der entstehenden Bestandesränder und nach der Dauer der Verjüngungszeiträume (siehe zum Beispiel EBERHARD 1914 oder PHILIPP 1926). Dadurch, daß das Saumverfahren schematisch vorgeht und nur schwer an Bedürfnisse des Holzmarkts anpaßbar ist, hat es keine große Bedeutung in der Praxis erlangt (siehe Dengler 1992 в und BuRschel und Huss 1997).

Im Schirmschlagverfahren werden gleichaltrige Bestände in mehreren Schritten genutzt, so daß der Bestand natürlich verjüngt werden kann. Es entstand in buchenbestockten Mittelgebirgen, und 1791 wurden von Harting "Generalregeln“ für diese Form der Bewirtschaftung beschrieben (HARTING 1991). Es wird vornehmlich in Buchenbeständen, aber auch in Mischbeständen aus Fichte, Buche und Tanne verwendet. In Mastjahren wird bei diesem Verfahren ein sogenannter Besamungshieb durchgeführt, um die Verjüngung einzuleiten. Ihm kann eventuell ein Vorbereitungshieb vorausgehen, durch den der Bodenzustand verbessert werden soll. Durch Lichtungshiebe, die sich dem 
Besamungshieb anschließen, wird die Entwicklung der Verjüngung gesteuert. Der Rest des Altbestandes wird abschließend mit einem Räumungshieb entnommen. Die Bestandesfläche wird beim klassischen Schirmschlagverfahren im Gegensatz zu Lochoder Femelhieben gleichmäßig aufgelichtet. In Loch- und Femelhieben werden Bäume nicht ganzflächig, sondern gruppen- und horstweise entnommen (siehe zum Beispiel Gayer 1882 oder VANSELow 1949). Dem ersten Femelhieb, der auch dazu dienen kann, den Bestand über schon vorhandener Verjüngung aufzulichten, folgen Rändelungshiebe, die die Lücken im Altbestand vergrößern. Der Räumungshieb schließt die Erntephase ab (BURSCHEL UND Huss 1997).

Die verschiedenen Schirmschlagverfahren können auch mit dem Saumschlagverfahren kombiniert werden (so genannte kombinierte Verfahren wie der „Kombinierte bayerische Femelschlag“ (siehe DeNGLER 1992 B). Die Varianten des Schirmschlags unterscheiden sich in Größe und Form der freigestellten Fläche und in ihren Verjüngungszeiträumen. Da sich diese Verfahren über einen längeren Zeitraum erstrecken, kann die Verjüngung ungleichaltrig sein. Die Ungleichheiten in der Verjüngung verwischen in der Regel im Laufe der Jahrzehnte. Falls dies nicht geschieht, kann der Bestand in einen Gruppenplenterwald übergehen.

Bei der Plenterwaldwirtschaft verschwimmt die Grenze zwischen Jungwuchspflege, Läuterung, Durchforstung und Endnutzung. Die Endnutzung von Stämmen muß neben dem Ertragsgedanken auch immer die erzieherische und verjüngende Wirkung berücksichtigen. Zur Abgrenzung der Endnutzung kann einzig die Stärke der entnommenen Bäume herangezogen werden, so daß entnommene zielstarke Bäume als endgenutzt gelten.

Die Zielstärkennutzung (Haniel Und Kleinschmit 1992 und Reininger 1987) spielt auch in der Naturgemäßen Waldwirtschaft neben den schon beschriebenen kahlschlagsfreien Endnutzungsmaßnahmen (zum Beispiel Femelschlag oder Schirmschlag) eine große Rolle, da besonderes Gewicht auf die Erzeugung von qualitativ hochwertigem Starkholz gelegt wird. Das Qualitätsholz kann relativ marktangepaßt entnommen werden, sobald die qualitäts- und bedarfsgerechte Zielstärke der Stämme erreicht ist. So fallen bei der Zielstärkennutzung im Vergleich zu Kahlschlagsvarianten relativ geringe Erntemassen an.

\section{Jungwuchspflege}

Jungwuchspflege werden alle Maßnahmen genannt, die vom Zeitpunkt der gesicherten Verjüngung bis zum Dickungsschluß bei ca. $2 \mathrm{~m}$ Oberhöhe zur Erziehung eines qualitativ hochwertigen Bestandes notwendig sind (siehe auch Bonnemann 1967, KRAmer 1976). Sie wird angewendet, um die Baumartenzusammensetzung und die Dichte des Jungwuchses zu beeinflussen und um eine erste Qualitätsauswahl zu treffen. Man unterscheidet Einzelbehandlung, Reihenbehandlung und Ganzflächenbehandlung. Einzel- und Reihenbehandlungen werden meist mechanisch durchgeführt, für die Fläche gibt es die Möglichkeit der chemischen Behandlung. Diese wird aber wegen der Einschränkung des Einsatzes von chemischen Mitteln im Wald immer weniger verwendet. Generell neigt auf Freiflächen erwachsene Verjüngung eher zu starker Differenzierung, auf die steuernd eingegriffen werden muß. Bei unter Schirm erwachsener Verjüngung ist das waldbauliche 
Ziel oft auch ohne Jungwuchspflege zu erreichen, und außerdem ist der Erhalt ökologisch wertvoller Nebenbaumarten im Rahmen vieler Waldbaukonzepte ausdrücklich erwünscht. Im Dickungsalter sollte daher nur eingegriffen werden, wenn es zur Förderung der Stabilität, Qualität oder Mischungsregulierung unbedingt erforderlich ist (siehe zum Beispiel SpelLmann 1996).

\section{Läuterung}

Unter Läuterungen werden Durchforstungseingriffe im Dickungsalter (vom Bestandesschluß bis um Eintreten der natürlichen Astreinigung) verstanden. Man unterscheidet negative von positiver Bestandesauslese (siehe BonNEMANN 1967, KRAMER 1976). Es gibt die Möglichkeit des mechanischen Aushiebs oder der Ringelung (nur im Laubholz).

Hauptzweck der Läuterungseingriffe ist es, dem angestrebten Waldentwicklungsziel näherzukommen, in dem der in dieser Phase des Bestandeslebens einsetzende, starke Ausscheidungsprozeß gesteuert wird. Einzelaspekte bei der Durchführung von Läuterungen sind:

- Erhalt der Mischbaumarten (zum Beispiel Förderung der Mischbaumart Buche gegen eine starke Fichtennaturverjüngung)

- Wachstums- und Qualitätsförderung durch Entfernung von Protzen, Zwieseln, krummwüchsigen oder beschädigten Stämmen zugunsten von Wertholzanwärtern (besonders für Lärche, Kiefer und Eiche)

- Zurückdrängung von dienenden Schattbaumarten, um die Vorwüchsigkeit der Lichtbaumarten zu erhalten (zum Beispiel bei Mischbeständen aus Buche und Lärche/ Edellaubholz). Die Kronen der Lichtbaumarten werden gefördert und die Zweischichtigkeit der Bestände wird erhalten

- Förderung der Stabilität von gering differenzierten Jungbeständen mit hoher Bestockungsdichte

- die Feinerschließung eines Bestandes kann mit einem Läuterungseingriff verbunden werden

Derzeit rückt die Negativauslese bei Läuterungen zu Gunsten von Ausleseläuterungen immer mehr in den Hintergrund. Unter dem Begriff der Ausleseläuterung versteht man eine Positivauslese, die zur Förderung von Z-Baum-Anwärtern der Kraft'schen Baumklassen 1 und 2 verwendet wird. Dieses Verfahren setzt zum Ende des Dickungsstadiums beim Übergang zur Stangenholzphase an. Es hat den Vorteil, daß die Erstdurchforstung ebenfalls zu einem späteren Zeitpunkt einsetzen kann und daß das Betriebsergebnis dieser Durchforstung durch den vermehrten Anfall stärkeren Holzes günstiger ausfällt. Zudem kommen auch bei einer Positivauslese die oben genannten Punkte (Förderung von Mischbaumarten, der Stabilität, der Qualität usw.) zum Tragen. 
Da kein oder nur unwesentlich verwertbares Derbholz anfällt und Schwachholz sich nur selten kostendeckend verwerten läßt, sollte der Läuterungsbedarf in jedem Fall kritisch geprüft werden.

Versäumnisse in der Jugend können schlecht durch Durchforstungseingriffe ausgeglichen werden. Doch trotz einer Unterlassung direkter steuernder Eingriffe ist eine aktive Steuerung möglich. Die Selbstdifferenzierung und -qualifizierung im Nachwuchs können durch die Überschirmungsverhältnisse so gelenkt werden, daß Läuterungseingriffe in vielen Fällen nicht mehr erforderlich sind (siehe auch Speldmann 1994в Spellmann 1996).

\section{Wertästung}

Bei der Ästung werden Zweige von stehenden Bäumen entfernt, um die Holzqualität des behandelten Baums durch die Produktion von astfreiem Holz im Erdstammstück zu fördern, (siehe auch BonNEMANN 1967). Es werden bei der Wertästung verschiedene mechanische Techniken angewendet, bei denen manuelle, pneumatische oder maschinelle Sägen verwendet werden können. Man unterscheidet Trockenästung (zum Beispiel bei Fichte) von Grünästung (zum Beispiel bei Tanne, Douglasie und Kirsche).

Die Entfernung von Ästen kann die natürliche Astreinigung vorwegnehmen oder sie ersetzen. Die Notwendigkeit zur Ästung ist bei angemessener Steuerung der Überschirmung seltener gegeben. Sie wird besonders bei Baumarten, die zur Totasterhaltung neigen (zum Beispiel Douglasie und Kirsche), angewendet, soweit der Aufwand und die Risiken, die mit der Ästung verbunden sind nicht zu hoch sind. Solche Risiken bestehen zum Beispiel bei der Lärchenästung, da sie die Vermehrung des Lärchenborkenkäfers unterstützen kann. Auch bei der Fichte bestehen Produktionsrisiken, so daß zu ästende Bestände einer besonders gründlichen Auswahl unterliegen sollten. In erster Linie wird die Wertästung an Nadelbäumen angewendet, da Laubbäume sich (bei geeigneter waldbaulicher Behandlung) in ausreichendem Maße selbst reinigen. Eine Ausnahme bilden hier Pappel und Kirsche. Besonders letztere kann nur unter Anwendung von Ästungsmaßnahmen zur Wertholzproduktion verwendet werden.

Generell sollten nur leistungsstarke und stabile Bestände geastet werden, da sich der zusätzliche Aufwand durch einen entsprechend höheren Wertholzanteil rentieren muß. Beschreibungen und Arbeitshinweise zur Wertästung finden sich zum Beispiel in Buschel und Huss 1997, Niedersächsische LandesforstVerwaltung 1995 oder Dengler 1992.

\section{Durchforstung}

Unter dem Begriff Durchforstung wird ein Pflegehieb im Stangen- oder Baumholz verstanden. Dabei wird eine Durchforstung bei einem BHD von $7 \mathrm{~cm}$ bis $20 \mathrm{~cm}$ (Stangenholz) als Jungdurchforstung und eine Durchforstung bei Bäumen, deren BHD über $20 \mathrm{~cm}$ liegt (Baumholz), als Altdurchforstung bezeichnet, (siehe auch BonNEMANN 1967, KRAmeR 1976). Oft wird das Wort „Vornutzung“ als Synonym für Durchforstung verwendet. Eine Durchforstung hat einerseits die Nutzung von Stämmen und gleichzeitig die Qualifizierung des verbleibenden Bestandes zum Ziel. Die klassischen Durchforstungsarten wurden im Zuge des naturnahen Waldbaus in den letzten 
Jahrzehnten abgewandelt und erweitert. Derzeit stehen bei Durchforstungen die Förderung von Wertleistung, Stabilität und Bestandesstruktur, aber auch die Kostensenkung im Vordergrund, (siehe auch Buschel und Huss 1997, Speldmann 1996).

Durchforstungen unterscheiden sich durch ihre Intensität, Durchforstungsstärke und ihre Art (Assmann 1961, S. 208-214). Alle drei Komponenten richten sich nach dem Produktionsziel und den Vorraussetzungen, die der betrachtete Bestand mit sich bringt (zum Beispiel Baumartenzusammensetzung, Schichtung, Ertragsniveau oder Qualität). Die Intensität ist als Zeitpunkt des ersten Eingriffs in Kombination mit der Häufigkeit der Wiederkehr definiert. Beides kann je nach Baumart, Pflegebedürftigkeit und Produktionsziel differieren und alter-, höhen- oder durchmesserabhängig festgelegt werden. Die Durchforstungsstärke orientiert sich an Stammzahl, der Grundfläche oder dem Volumen. Baumzahlleitkurven sind Beispiele für baumzahlorientierte Durchforstungsanweisungen, die baumartengetrennt entwickelt wurden (siehe zum Beispiel für Fichte (Abetz 1975), Douglasie (Kenk und HRAdETZKy 1985) oder Eiche (Kenk 1984). Grundflächenbasierte Wachstumszusammenhänge werden zum Beispiel von Assmann dargestellt (Assmann 1961, S. 292-299). Im folgenden werden verschiedene Durchforstungsarten vorgestellt. Dabei wird zwischen Hoch-, Gruppen- und Niederdurchforstungen unterschieden.

Hochdurchforstungen streben einen zwei- oder mehrschichtigen Bestandesaufbau an und bereiten meist eine Zielstärkennutzung vor (siehe zum Beispiel REININGER 1987). Es werden jeweils die im waldbaulich-ökologischen Sinne besten Bäume der herrschenden Schicht gefördert; Kriterien sind dabei Qualität, Stabilität, Massenentwicklung und Baumartendurchmischung. Das Ziel der Hochdurchforstung ist eine maximale Wertentwicklung und Stabilisierung ausgesuchter Individuen (Z-Bäume) aus der Oberschicht (SCHÄDELIN 1934). An diese Form der Wertholzproduktion schließt sich in der Regel eine Zielstärkennutzung (siehe „Endnutzungsmaßnahmen“) an. Damit sich diese Bäume optimal entwickeln können, werden sie in einer sogenannten „Auslesedurchforstung“ (LEIBUNDGUT 1968) von weniger wertvollen Bedrängern aus derselben Schicht befreit. Dabei kann eine Unter- oder Zwischenschicht aus schattentoleranten Bäumen erhalten bleiben. Diese trägt zur Erhaltung der Schaftreinheit der zu fördernden Bäume oder auch zur Steuerung der Verjüngung bei, indem unerwünschte Bodenvegetation verhindert wird. Die verschiedenen Hochdurchforstungsvarianten unterscheiden sich darin, ob die Z-Bäume dauerhaft markiert werden, wie viele Bäume gefördert werden, mit welcher Stärke die Eingriffe erfolgen und in der Wahl des Eingriffszeitpunkts sowie des Eingriffsintervalls.

Es können statt einzelner Individuen auch Gruppen gefördert werden (BussE 1935, KATO 1973, Kato und Mülder 1979, Kato und Mülder 1983 und Kato und Mülder 1988), wodurch die in Naturwäldern beobachtete Klumpung starker Bäume nachgeahmt wird. Die gängige Form der Einzelbaumauslese und die zu erwartende Entwicklung der Z-Bäume wird zum Beispiel von Abetz und Klädtke beschrieben (ABETZ 1980, ABETZ und KLÄDTKE 2002, KLÄDTKE 1993). Eine Variante, bei der nur relativ wenige Individuen nach einer langen Phase der Selbstdifferenzierung besonders stark gefördert werden, stellt ein Konzept zur naturnahen Erzeugung von starkem Wertholz dar, das von Wilhelm et al. beschrieben wird (WILHELM, LeTter UND EDER 1999) (siehe auch UTSCHIG 2000). Vorbild für 
diese Art der Wertholzproduktion sind französische und belgische Mittelwälder, die durchgewachsen sind sowie dänische Pflegestrategien.

Bei Niederdurchforstung werden vorrangig beherrschte Bestandesmitglieder entnommen und dadurch ein einschichtiger Bestandesaufbau angestrebt. Das Ziel ist eine maximale Volumenleistung und kollektive Stabilität. Man unterscheidet verschiedene Eingriffsstärken (schwach, mäßig, stark oder Lichtung) und Eingriffszeitpunkte. Auch der Übergang von einer Eingriffsstärke zu einer anderen kommt zum Beispiel in der gestaffelten Durchforstung oder der Schnellwuchsdurchforstung vor. Je schwächer eingegriffen wird, desto weniger wird auf die Wert- und Qualitätsentwicklung der herrschenden Bäume Einfluß genommen. Mit zunehmendem Grad der Niederdurchforstung wird immer stärker in die herrschende Schicht eingegriffen und somit auch ihre Qualität und Massenentwicklung direkt beeinflußt. Da einerseits einschichtige Bestände nicht den heutigen Zielvorstellungen entsprechen, andererseits die Entnahme unter- und zwischenständiger Bäume hohe Kosten verursacht und nur einen geringen Effekt auf die Qualität des verbleibenden Bestandes ausübt, verliert die Niederdurchforstung zunehmend an Bedeutung. Nur in einigen Bestandessituationen bleibt ihre Relevanz erhalten, zum Beispiel wenn sich der Unterstand auch bei Kronenöffnung nicht erhalten läßt oder die Wuchsleistung der herrschenden Bäume durch den Unterstand (auf sehr trockenen Standorten) gemindert wird (BuSCHEL UND Huss 1997).

Es können auch Hoch- und Niederdurchforstungen in Kombination verwendet werden. Ein Beispiel dafür sind die Baumzahlleitkurven nach Abetz (AвETz 1975), die für die Fichte zum Beispiel eine Auslesedurchforstung bis zu einer Oberhöhe von 23m und anschließende Niederdurchforstung vorsehen. Durchforstungen nach der Methode der Baumzahlleitkurven bereiten den Bestand in der Regel für einen anschließenden Kahlschlag vor, indem sie zu einer einheitlichen Durchmesserstruktur beitragen (DENGLER 1992 в).

\subsection{Die Eignung planungsunterstützender Instrumente}

Im Zuge des praktizierten Waldumbaus werden alte Waldbewirtschaftungskonzepte von neuen abgelöst. Da getroffene Entscheidungen im Wald langfristige Auswirkungen haben und eine Entscheidungsfindung gründlich und auf fundierten Annahmen basierend getroffen werden muß, besteht Bedarf an planungsunterstützenden Instrumenten, um die Realisierbarkeit von Planzielen zu prüfen, sie zeitlich festzulegen und ihre Umsetzung meßbar zu machen (siehe SPEIDEL 1972). Da Waldbaustrategien die Multifunktionalität des Waldes berücksichtigen (siehe auch Spellmann, Hillebrand und Cornelius 2001 und SpelLmANn ET AL. 2004), sollten auch die Entscheidungen multikriteriell getroffen werden können. Neben den Baum- und Bestandeswerten werden auch zunehmend Struktur-, Ökologie- und sozioökonomische Daten benötigt.

Die Vielfalt der in Frage kommenden Bewirtschaftungsformen (siehe Kapitel 2.2.3) führt neben der Langfristigkeit der nötigen Prognosen zu Schwierigkeiten bei der Wahl eines geeigneten Instruments. Daher sollen in dieser Arbeit Eingriffsmodelle mit Wachstumsmodellen verbunden werden, um Effekte verschiedener Bewirtschaftungsformen transparent zu machen. Ein solches Gesamtmodell kann dann zu 
einem geeigneten Analysewerkzeug werden, wenn es in ein praktikables EDV-Programm, einen Waldwachstumssimulator, eingebettet wird. Waldwachstumssimulatoren, die in der Lage sind, Prognose- und Szenariorechnungen umzusetzen und Handlungsalternativen abzubilden, können bei Entscheidungen im Waldbau als wichtiges Instrument dienen (siehe zum Beispiel in Spellmann 2003 oder Mosandl und Felbermeier 2003).

\subsubsection{Betrachtung verschiedener Waldwachstumsmodelle}

Um bei der Waldbewirtschaftung auf unterstützende Mittel zurückgreifen zu können, werden seit Bestehen der Forstwissenschaft Wachstumszusammenhänge untersucht. Sie können in Form von Graphiken, Tabellen, Funktionen oder Modellen präsentiert werden.

Es gibt vielerlei Ansätze, das Ökosystem „Wald“ darzustellen. Sie unterscheiden sich je nach der Zielsetzung, mit der sie entwickelt wurden

- im Maßstab ihrer Betrachtungsebene und dem damit verbundenen Komplexitätsgrad (siehe Abbildung 2.3.1.a).

- $\quad$ von dem prognostizierten Zeithorizont her (siehe Abbildung 2.3.1.a).

- in der verwendeten Datengrundlage zur Erstellung und Validierung, die ihren Einsatzbereich definiert.

- in ihrer Form der Darstellung bzw. der Ergebnisausgabeoptionen, die an die Anwender und das Anwendungsgebiet angepaßt sind.

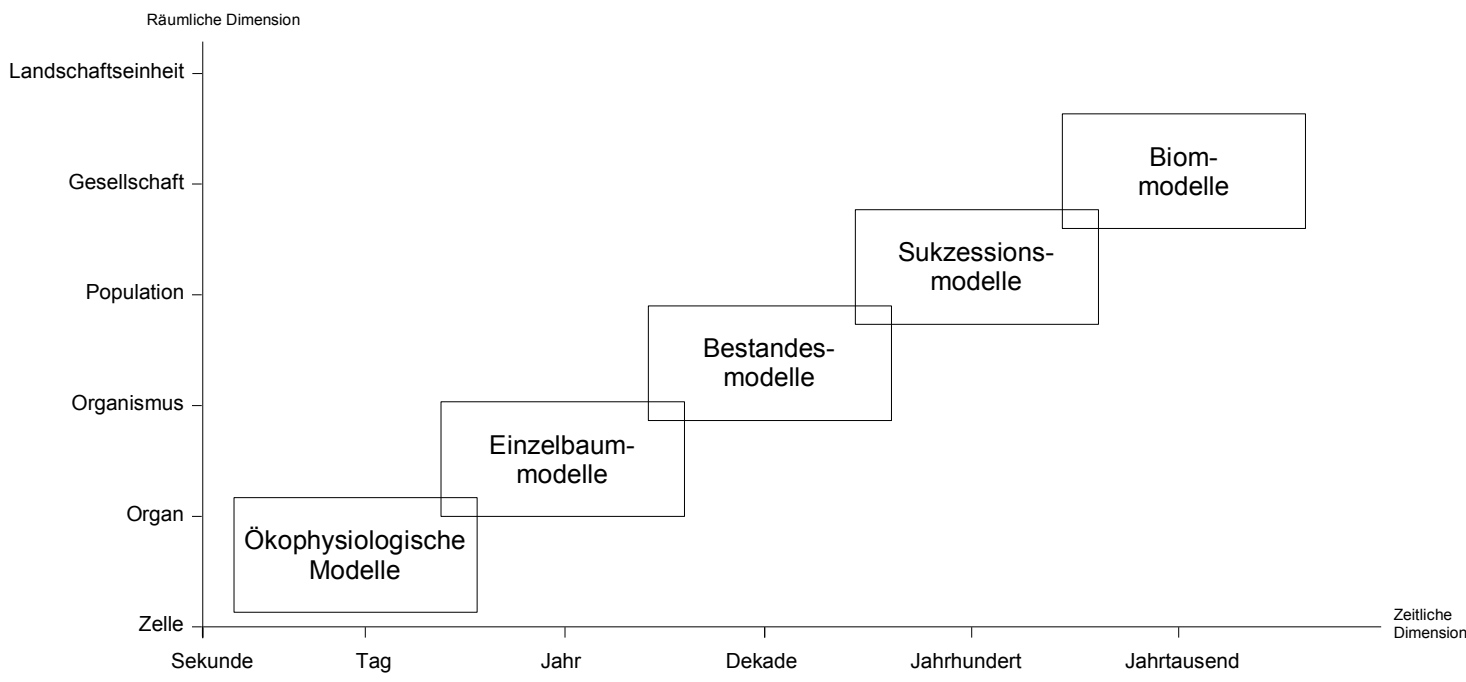

Abbildung 2.3.1.a: Von ökophysiologisch basierten Prozeßmodellen über Managementmodelle bis zu Sukzessions- und Biommodellen nimmt die räumliche und zeitliche Aggregation bei der Nachbildung der Prozesse und Strukturen zu (nach PRETZSCH 2001).

Keines der Modelle kann durch eines der Anderen ersetzt werden. Die verschiedenen Ansätze erlauben die Betrachtung des Objekts Wald aus unterschiedlichen Blickwinkeln und können modellspezifische Erkenntnisse vermitteln. Ihre Einteilung lehnt sich an die Veröffentlichung von Pretzsch (PRETZsCH 2001) an. 
Damit die in Kapitel 1.2 vorgestellten Ziele verwirklicht werden können, werden die vorgestellten Waldwachstumsmodelle hier nach einer kurzen Vorstellung auch unter dem Gesichtspunkt betrachtet, ob sie als Grundlage für ein planungsunterstützendes Instrument zum Vergleich forstlicher Managementstrategien dienen können.

Zu beachten ist,

- daß ein Modell, für das die Eingangsparameter nicht vorliegen oder nur mit einem erheblichen Aufwand erhoben werden können, lediglich für den Einsatz in der Forschung, aber nicht für den Einsatz in der Praxis geeignet ist.

- daß verschiedenste waldbauliche Ausgangssituationen vorliegen und das Modell insbesondere in der Lage sein sollte, ungleichaltrige Mischbestände abzubilden. Die Nutzer und Besitzer sehen ihre Anforderungen an den Wald nicht mehr in Reinbeständen erfüllt, (siehe auch SPIEKER ET AL. 2004 für Fichte). Gerade Reinbestände, die nicht standortgemäß sind, werden als risikobehaftet im ökologischen und ökonomischen Sinn gesehen, da sie als anfälliger für Schäden durch Insektenmassenvermehrungen oder Sturm gelten und Mischbeständen eine höhere Elastizität gegenüber Störungen zugeschrieben wird (Отто 1990). Außerdem fehlt gleichaltrigen Reinbeständen die nötige Flexibilität in bezug auf sich ändernde Marktanforderungen.

- daß zum Vergleich von Waldbaustrategien unterschiedliche forstliche Eingriffe abgebildet werden müssen.

- daß der Planungshorizont überschaubar bleiben muß, er es aber gleichzeitig ermöglichen sollte, die Auswirkungen verschiedener Waldbaukonzepte sichtbar zu machen.

- daß die Auswirkungen der Behandlungen anhand von multifunktionalen Kriterien transparent dargestellt werden sollen.

\section{Ökophysiologische Prozeßmodelle}

Ökophysiologische Prozeßmodelle ${ }^{4}$ eignen sich in besonderem Maße dafür, das Verständnis von Waldökosystemen zu fördern. Die ökophysiologischen Prozeßmodelle gehören in Abgrenzung zu deskriptiven Modellen, die auf statistischen Daten und Korrelationen oder Regressionen basieren, zu der Gruppe der erklärenden Modelle. Im Vordergrund stehen hier Strukturen und Prozesse, die die Mechanismen abbilden, welche der Entwicklung zugrunde liegen. Sie prognostizieren das Verhalten des Ökosystems Wald als Summe von Teilprozessen wie chemischen Reaktionen (zum Beispiel im Boden oder im Blatt), Stoffkreisläufen, Allokations- oder Organbildungsprinzipien. Im Prinzip ist dieser Modelltyp sowohl für Misch- als auch Reinbestände geeignet. Aus den Ergebnissen lassen sich eine Vielzahl an Parametern ableiten (wie zum Beispiel Strukturparameter, Stoffflüsse oder Volumina und Massen einzelner Elemente (zum Beispiel $\mathrm{CO}_{2}$ )). Allerdings

4 Beispiele für ökophysiologische Modelle sind bei Bartelink 2000, Landsberg UNd WarIng 1997, Korol, Milner und Running 1996, Hauhs, Kastner-Maresch und Rost-Siebert 1995, Bossel 1994 oder SiEVÄNEN 1993 zu finden. 
reicht der Stand der Forschung oft nicht aus, um involvierte Teilprozesse vollständig abzubilden, und es fehlen Informationen, um aus der Summe der Teilprozesse auf das Gesamtsystem schließen zu können. Eine weitere Schwierigkeit beim Einsatz der ökophysiologischen Modelle liegt in den Ansprüchen, die an die Datengrundlage gestellt werden. Zur Initialisierung und Prognose der Prozesse sind oftmals detaillierte Informationen notwendig, die bei gängigen Waldmonitoringsystemen nicht aufgenommen werden. Der Detaillierungsgrad dieses Modelltyps macht die Simulationen besonders rechenintensiv. Die Differenziertheit der Ergebnisse übersteigt die Anforderungen, die an ein Modell zum Vergleich der mittel- bis langfristigen Auswirkungen forstlicher Managementstrategien gestellt werden.

\section{Einzelbaumbasierte Modelle}

In Nordamerika entstanden in den 70er Jahren die ersten Modelle, die das Bestandeswachstum auf der Grundlage der Entwicklung von Einzelbäumen berechneten. Zunächst galten die verwendeten Funktionen nur für gleichaltrige Reinbestände ${ }^{5}$. Aus der Notwendigkeit heraus, auf die sich ändernden Ansprüche an ein Bestandesmodell reagieren zu können, wurden die einzelbaumbasierten Wuchsmodelle kontinuierlich weiterentwickelt. Monserud und Ek waren die ersten, die in der Mitte der 70er ungleichaltrige Rein- und Mischbestände fortschrieben ${ }^{6}$. Als Datengrundlage für die Entwicklung von Einzelbaummodellen werden Baum- und Bestandesdaten aus Mehrfachmessungen (Zeitreihen) oder Einfachmessungen zur Bildung unechter Zeitreihen verwendet (PRETZSCH ET AL. 2006). Da auch in Skandinavien und Zentral-Europa vielerorts eine sehr gute Datengrundlage für die Entwicklung von Wuchsmodellen vorliegt (siehe voN Gadow und Hul 1999 und PRETZsCh 2001), entstanden auch dort seit Anfang der 90er Jahre zahlreiche Arbeiten?

Die Modelle bilden die Entwicklung des Einzelbaums in Abhängigkeit von baumspezifischen Wuchsbedingungen als Summe aus Konkurrenz- und Umgebungseinflüssen ab (siehe Abbildung 2.3.1.b). Dabei sind distanzabhängige Modelle von distanzunabhängigen Modellen zu unterscheiden. Distanzunabhängige Wachstumsmodelle ermitteln die Konkurrenzsituation mit Hilfe der durchschnittlichen Entfernung zwischen den Bäumen. Distanzabhängige Modelle beziehen die Position des Baum in Relation zu seinem Nachbarn mit ein. Bei solchen Modellen werden die Koordinaten der Einzelbäume benötigt. Einmal festgelegte Positionen werden beibehalten, so daß Auswirkungen ungleichmäßiger Verteilungen oder Klumpungseffekte mit in die Modellierung einbezogen werden können und Strukturparameter ableitbar sind. Je größer

5 Siehe zum Beispiel Newnham 1964, Lee 1967, Mitchell 1969, Lin 1970, Bella 1971, Arney 1972, Stage 1973, Mitchell 1975 und Daniels und BuRkhart 1975.

6 Siehe EK Und Monserud 1974 und Monserud 1975. Es folgten Veröffentlichungen von WYKoff, Crookston und Stage 1982, Wensel und Daugherty 1984, van Deusen und Biging 1985, Wensel und Koehler 1985 sowie Burkhart et al. 1987.

7 Siehe zum Beispiel Sterba 1983, Pukkala 1987, Pukkala 1988, Pukkala 1989, Eckmüllner und Fleck 1989, Pretzsch 1992, Hasenauer 1994, Nagel 1995, Sterba et al 1995, Biber 1996, Nagel 1996, Monserud und Sterba 1996, Kahn und Pretzsch 1997, Pretzsch 1997, Nagel 1999, Hasenauer 2000, Pretzsch 2001, Pretzsch et al. 2002, Pretzsch, Biber und Durský 2002, Nagel, Albert und Schmidt 2002 oder Pretzsch, Biber und Durský 2002. 
die Untersuchungseinheit gewählt wird, desto mehr wirkt sich die Struktur des Bestandes auf die Ergebnisse aus (siehe auch STRUGEN in PRETZSCH 1993 oder Pommerening 2000).

Die gesamte Entwicklung eines Baums hängt von mehreren Funktionen ab. Ein Einzelbaumwachstumssimulator sollte zumindest aus Submodellen zum Durchmesserund Höhenwachstum, zur Mortalität und zur Verjüngung bestehen (siehe SöDERBERG UND Ledermann 2003 und Abbildung 2.3.1.b). Da Verjüngungs- oder Einwuchsmodelle Bestandteile des Gesamtmodells sind, ist es auch auf ungleichaltrige und mehrschichtige Bestände anwendbar, in denen zum Beispiel Einzelstammnutzungen durchgeführt werden. Ein Bestand wird in Einzelbaumwachstumsmodellen durch die Aggregation der Einzelbäume definiert. Dabei können ohne weiteres auch ungleichaltrige Mischbestände abgebildet werden. Je nach Entwicklungsstand des Modells können Durchforstungen, Störungseinflüsse und Klimaänderungen (siehe Abbildung 2.3.1.b) in die Gesamtentwicklung einbezogen werden.

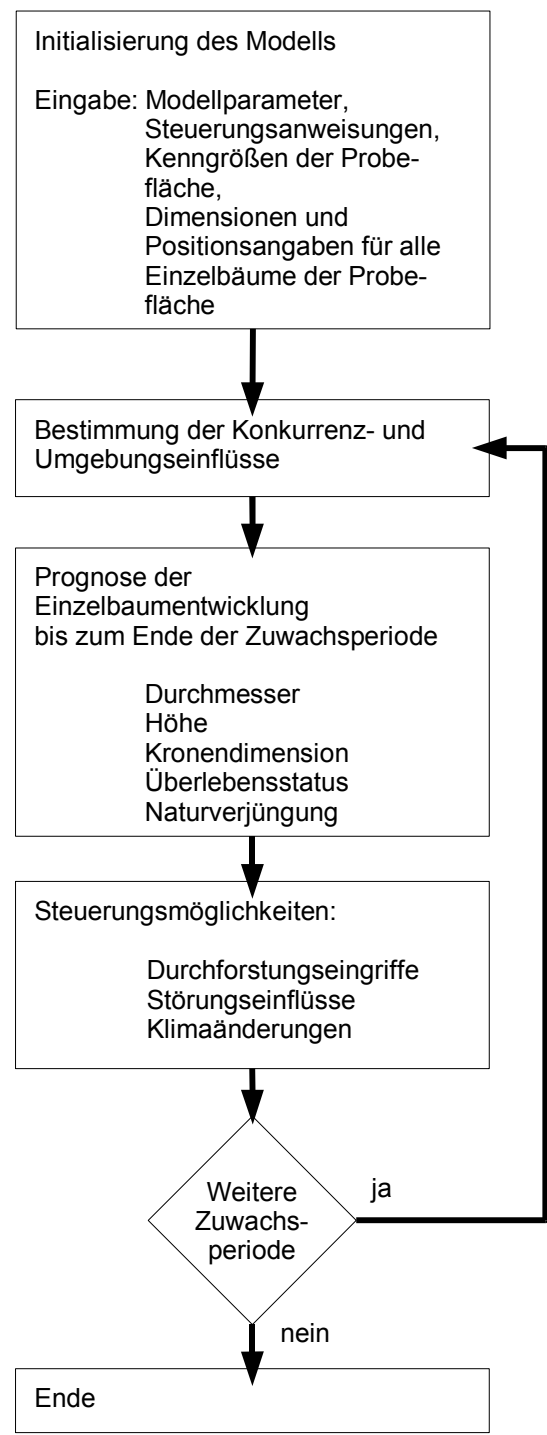

Abbildung 2.3.1.b: Simulationsprozeß bei positionsabhängigen Einzelbaummodellen in schematischer Darstellung (nach EK UND DUdEK 1980 in PRETZSCH 2001). 
Dadurch, daß einzelne Submodelle bei Verbesserung des Stands der Forschung ersetzt und erweitert werden können, ist diese Art der Waldwachstumsmodelle besonders flexibel und kann an aktuelle Fragestellungen im Waldbau angepaßt werden. Durch die detaillierte Betrachtungsweise der Kausalzusammenhänge können mit Hilfe ökophysiologischer Prozeßmodelle Ergebnisse einzelbaumbasierter Modelle untermauert und hinterfragt werden. Eine Mischung aus den beiden Modelltypen wird als Hybridmodelle bezeichnet.

Als Wachstumssimulatoren werden Einzelbaummodelle zu wirkungsvollen Instrumenten der Waldbauplanung (siehe zum Beispiel PRETZSCH UND KAHN 1995). Im Verbund mit einer graphischen Benutzeroberfläche veranschaulichen sie waldbauliche Möglichkeiten und können auch zu vielen verschiedenen Einsatzzwecken verwendet werden. Dursky stellt zum Beispiel die folgenden Verwendungsmöglichkeiten vor (siehe DuRSKY 2000):

- Ersatz von Ertragstafeln

- Erstellung spezieller Tafelwerke

- Waldbauliche Strategieplanung und Optimierung der Bestandesbehandlung

- Nutzungsplanung in der Forsteinrichtung

- Erweiterung des Begriffs Nachhaltigkeit

- Fortschreibung von Stichprobeninventuren auf Betriebs- und Landesebene

- Liquiditätsplanung auf Betriebsebene und Ermittlung von Abtriebswerten

- Auswirkungen von Klimaänderungen und Baumartenwahl

- Holzaufkommensprognose

- Sozioökonomische Sektoranalyse

- Schulung, Ausbildung, Lehre

\section{Bestandesdurchschnitts- und Summenwerte}

Die ältesten Modelle der Forstwirtschaft sind die Ertragstafeln ${ }^{8}$. Die ersten Tafelwerke wurden vor über 200 Jahren entwickelt. Mit innen werden dem Benutzer biometrische Durchschnitts- und Summenwerte auf Bestandesebene (wie dg, hg, h100, aber auch Grundfläche, Stammzahl oder Zuwachs) in Abhängigkeit vom Alter des Bestandes an die Hand gegeben. Sie werden den Bestandesmodellen zugeordnet (siehe Abbildung 2.3.1.a).

Grundlage solcher Prognosen sind Meßwerte aus Versuchsanlagen, die entweder graphisch oder statistisch ausgeglichen wurden. Die Bestände werden dabei Ertrags- oder Leistungsklassen zugeordnet, so daß standortsabhängige Unterschiede für das Wachstum

8 Frühe Beispiele dafür sind Tafeln von Harting 1795, Schwappach 1889, Schwappach 1890, von Guttenberg 1915, Gehrhardt 1930 und Wiedemann 1949. Später folgten unter anderen Assmann und Franz 1963, Bradley, Christie und Johnson 1966, Schober 1975, Wenk, Römisch und Gerold 1985, Bergel 1986 oder auch im letzten Jahrzehnt Lockow 1995, Lockow 1998. Die neueren Modelle sind EDV-gestützt. 
der Baumarten berücksichtigt werden können. Der Anwender ordnet den von ihm betrachteten Bestand einer Ertragsklasse zu, indem er die Auswahl der Tafel anhand von leicht zugänglichen Bestandesdaten wie Alter und Höhe in Kombination mit der Baumart trifft. Regionale Effekte bleiben dabei unberücksichtigt; meist wurden Ertragstafeln für größere Gebiete kalibriert.

Die Tafeln beziehen sich in der Regel auf die Entwicklung von vollbestockten Reinbeständen. Tafeln für Mischbestände, wie sie zum Beispiel von Bonnemann 1939, Wiedemann 1942 entwickelt wurden, setzten sich nicht durch, weil die Benutzer ihre spezifischen Mischbestände nicht durch diese Tafeln repräsentiert sahen (VON TEUFFEL ET AL. 2006). Wenn der Anwender Mischbestände betrachten möchte, so schreibt er in der Regel jede Baumart altersstufengetrennt fort, als stünde sie in einem gleichaltrigen Reinbestand. Der Bestandeswert ergibt sich dann durch Aufsummieren der nach den Grundflächenanteilen gewichteten Einzelwerte. Die Summe der Einzelwerte entspricht aber gerade in ungleichaltrigen Mischbeständen nicht dem tatsächlichen Bestandeswert. Eine weitere Einschränkung besteht bei der Nutzung von Ertragstafeln auch in der Bandbreite an Nutzungsarten, die von den Ertragstafeln wiedergegeben werden können. Weicht man von dem unterstellten Durchforstungskonzept (meist Niederdurchforstungen) ab, können die statischen Ertragstafeln die tatsächliche Entwicklung nicht mehr abbilden. Die Vielzahl der heutzutage verwendeten Eingriffsvarianten und Mischungsformen sind in einem Tafelwerk nicht mehr abbildbar, daher stellen sie kein geeignetes Instrument zum Vergleich forstlicher Managementstrategien dar.

Wie die Ertragstafeln wird in Standort-Leistungstafeln die Entwicklung von Bestandesmittelwerten dargestellt (PRETZSCH 2001). Allerdings sind sie nur für die zugrundeliegende Standorteinheit aussagekräftig. Das Programm STAOET (FRANZ 1968 in

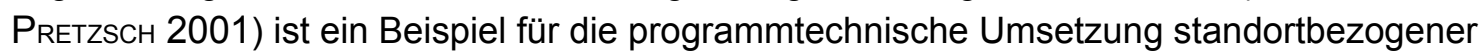
Leistungstabellierung. Es ist durch die Möglichkeit der Abbildung verschiedener Behandlungsprogramme wesentlich flexibler als klassische Ertragstafeln. Da die Darstellung von Durchschnitts- und Summenwerten jedoch generelle Einschränkungen bei der Auswertung von Auswirkungen aktueller Bewirtschaftungskonzepte nach sich zieht (zum Beispiel keine bestandesinternen Strukturparameter ausgewertet werden), genügt auch ein solches Programm den hier gestellten Anforderungen nicht.

\section{Stammzahlfrequenzmodelle}

Einen höheren Informationsgehalt als Bestandesmittelwertmodelle bieten Stammfrequenzmodelle, da sie neben der Information über den Gesamtbestand auch Häufigkeitsverteilungen von Bäumen über Teilkollektiven (meist stärkebezogen) abbilden.

$\mathrm{Zu}$ dieser Art von Modellen zählen stochastische Evolutionsmodelle, Differentialgleichungsmodelle und Verteilungs-Fortschreibungsmodelle (PRETZSCH 2001). Sie gehören wie die Bestandesdurchschnitts- und Summenwertmodelle zu den Bestandesmodellen (siehe Abbildung 2.3.1.a).

Suzukis stochastisches Entwicklungsmodell (das auch „Evolutionsmodell“ genannt wird) (SuzUKı 1971) wurde durch SLOBODA 1977 einem breiten forstlichen Publikum in Europa zugänglich gemacht. Das Modell basiert auf der Entwicklung von 
Durchmesserklassenverteilungen, die anhand der Übergangswahrscheinlichkeiten der Einzelbäume (im Rahmen des Markow-Prozesses) errechnet wird. Da die weitere Entwicklung aus der bisherigen Entwicklung vorausgesagt wird, setzt das Modell eine gleichbleibende Bestandesbehandlung voraus. Für eine Untersuchung der Auswirkung von Behandlungsalternativen scheint das Modell daher nur wenig geeignet.

Differentialgleichungsmodelle beschreiben Veränderungen von Waldzustandsgrößen wie zum Beispiel Stammzahlen, Grundflächen oder Vorräte. Die Veränderungsraten werden durch zum Beispiel regressionsanalytisch hergeleitete Funktionen beschrieben, die von ertragskundlichen Kennwerten des Ausgangsbestandes abhängen. Forstliche Eingriffe können durch die Reduktion der Stammzahl und der Grundfläche auf die weitere Entwicklung einwirken. Beispiele für solche Modelle kommen von ClutTer 1963, Moser und Hall 1969, Moser 1972 oder PienaAr und Turnbull 1973.

Bei der Verteilungsfortschreibung wird die Entwicklung eines Bestandes durch die Entwicklung der Baumdurchmesser- oder Baumhöhenverteilung charakterisiert. Diese Art von Modell wurde erstmals von CLUTTER UND BENNETT 1965 als Durchmesserverteilungsfunktionen für gleichaltrige Reinbestände veröffentlicht . Es schließen sich weitere Arbeiten an. Zum Beispiel haben HyINK UND MOSER 1983 das Modell an ungleichaltrige Reinbestände angepaßt und VON GADOW 1987 integrierte Durchforstungsvarianten in das Modell. Die Arbeit von Klädtke und Yue ist ein Beispiel für ein Verteilungsstufenmodell, das auf die Planungsunterstützung einer bestimmten Bewirtschaftungsform spezialisiert ist (siehe KLÄDTKE UND YUE 2003).

Stammzahlfrequenzmodelle bilden die Wanderungsbewegung der Stammzahl- und Durchmesserverteilungen ab. Dabei können sie zwar auf die Abbildung bestimmter Behandlungskonzepte spezialisiert sein, ihre Prognosemöglichkeiten reichen aber nicht aus, um die Modelle als Werkzeug zur Analyse der Auswirkung verschiedener Waldbewirtschaftungskonzepte auf die aktuelle waldbauliche Situation verwenden zu können. Durch die verwendete Betrachtungsebene der Verteilungen können dem Benutzer außerdem nur begrenzt multikriterielle Parameter an die Hand gegeben werden.

\section{Kleinflächen- und Biommodelle}

In Kleinflächenmodellen ${ }^{9}$ werden Kleinflächen mittlere Wuchsbedingungen zugeordnet, in Abhängigkeit von welchen sich die dort stockenden Bäume entwickeln. Der Bestand setzt sich aus einer Vielzahl von Kleinflächen zusammen, deren Größe der Ausdehnung der Standfläche eines Altbaums entspricht (siehe PRETZSCH 2001). Die Kleinflächen können durch Wechselwirkungen miteinander in Beziehung treten. Die Stärke dieses Modelltyps liegt in der Abbildung von Sukzessionsprozessen und der damit verbundenen Biomassenentwicklung. Auch Auswirkungen von Störungseinflüssen können berücksichtigt werden. Die von innen ausgegebenen Informationen zielen weniger stark auf die Anwendung in der Forstwirtschaft ab als Einzelbaum- oder Bestandesmodelle. Vielmehr soll die langfristige Sukzession und Biomassenproduktion unbehandelter Bestände auch

9 Der Kleinflächenmodellansatz wurde erstmals von WATT 1947 in die Forstwissenschaft eingebracht und auch von Bray 1956, Curtis 1959, Bormann und Likens 1979 a, Bormann und Likens 1979 B sowie SHugaRt 1984 aufgegriffen. 
unter veränderten Wuchsbedingungen vorhergesagt werden können. Diese zeitliche Ebene der Betrachtung und der Informationsschwerpunkt trifft also die Intention dieser Arbeit nur unzureichend.

Biommodelle, wie sie zum Beispiel von Box und Meentemeyer 1991 oder Prentice et AL.1992 vorgestellt werden, bilden im Rahmen der Klimaforschung die Korrelation von regionalem Klima und dem daraus langfristig (in Jahrhunderten bis zu Jahrtausenden) resultierenden Vegetationstyp als zu erwartende Lebensgemeinschaft (Biom) ab. Sie arbeiten mit einer größeren Flächenbezugsskala auf einem weiteren Zeithorizont als Kleinflächenmodelle und entfernen sich damit noch weiter von den Anforderungen, die im Rahmen dieser Arbeit an eine planungsunterstützende Software gestellt werden.

\subsubsection{Einzelbaumwachstumssimulatoren als geeignete Instrumente zum Vergleich forstlicher Managementstrategien}

Ein Waldwachstumsmodell kann zu einem geeigneten Instrument zum Vergleich forstlicher Managementstrategien werden, wenn es folgende in Kapitel 2.3.1 vorgestellten Kriterien erfüllt: Es sollte...

- auf in der Forstwirtschaft gängigen Daten basieren.

- $\quad$ in der Lage sein, verschiedenste waldbauliche Ausgangslagen fortzuschreiben.

- unterschiedliche forstliche Eingriffe abbilden können.

- $\quad$ einen angemessenen Zeithorizont repräsentieren.

- Transparenz über die Auswirkungen von Behandlungsalternativen anhand von multifunktionalen Kriterien schaffen.

In dieser Arbeit wird ein einzelbaumbasierter Waldwachstumssimulator erweitert und eingesetzt. Im folgenden werden die Vorzüge einzelbaumbasierter Waldwachstumssimultion noch einmal eingehend beschrieben.

\section{Eingangsdaten}

Von Einzelbaumwachstumssimulatoren wird die Entwicklung von Einzelbäumen in der Regel bestandesweise prognostiziert. Liegen dem Benutzer keine Einzelbauminformationen vor, sind die Simulatoren in der Lage, aus Bestandes- bzw. Verteilungsmittelwerten (wie zum Beispiel Mittelhöhe und Alter), die im Zuge der Forsteinrichtung aufgenommen werden, Einzelbaumdaten zu generieren. Wurden nicht alle relevanten Informationen (wie zum Beispiel der mittlere Brusthöhendurchmesser) aufgenommen, können diese mit einfachen Funktionen ergänzt werden. Positionsabhängige Modelle sind in der Lage, den generierten Einzelbäumen mit Hilfe von Verteilungsalgorithmen Koordinaten zuzuweisen. Im Vergleich zu zum Beispiel ökophysiologischen Modellen benötigen sie also nur relativ grobe, leicht zugängliche Eingangsdaten. 


\section{Abbildung und Fortschreibung der waldbaulichen Ausgangslage}

Um eine waldbauliche Ausgangssituation realitätsnah abzubilden, muß der Simulator mit ungleichaltrigen Mischbeständen umgehen können. Durch den Aufbau des Fortschreibungsobjekts „Bestand“ aus Einzelbäumen können ungleichaltrige Mischbestände ohne weiteres fortgeschrieben werden, sofern Funktionen für die vorkommenden Arten vorliegen. In der Regel sind in die Simulatoren zumindest getrennte Funktionen für die Baumarten Buche, Fichte, Eiche, Kiefer und Lärche integriert. Da ein Einzelbaumsimulator nicht nur, wie zum Beispiel die Ertragstafeln, einen Ausschnitt aus dem Bestandesleben abbilden kann, sondern mit Hilfe von Verjüngungsroutinen auch über die Endnutzung hinaus prognostiziert, sind alle Voraussetzungen für die Fortschreibung ungleichaltriger Mischbestände gegeben.

\section{Abbildung forstlicher Eingriffe}

Die im Einzelbaummodell verwirklichte Rückkopplungsschleife zwischen Bestandesstruktur, Zuwachs, Baumzustand zurück zur Bestandesstruktur bietet ideale Voraussetzungen, um die Auswirkungen von forstlichen Eingriffen mehrdimensional nachzubilden (siehe Abbildung 2.3.2.a).

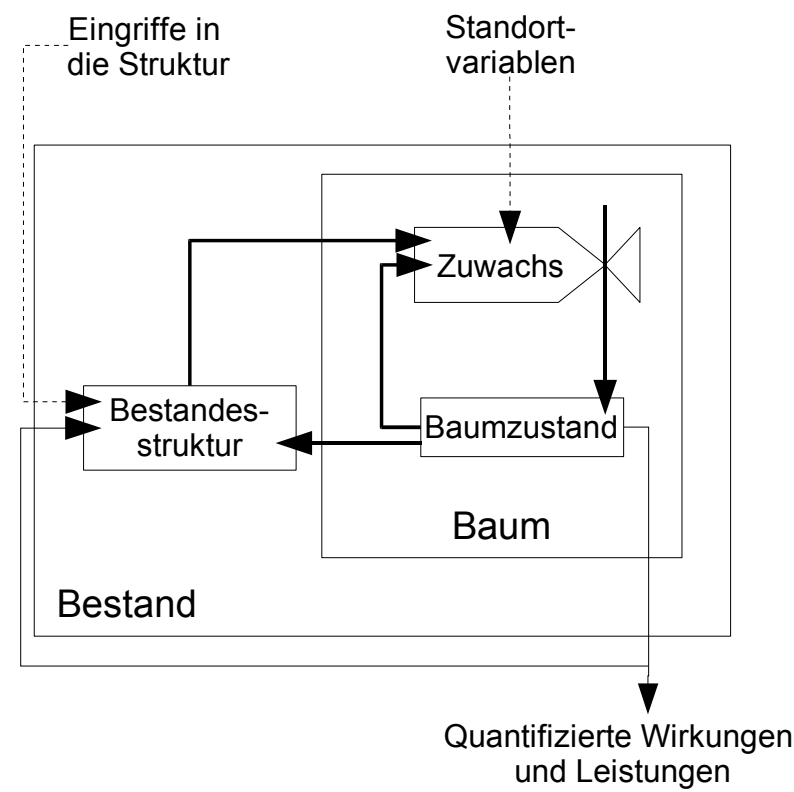

Abbildung 2.3.2.a: System mit den Ebenen Bestand und Baum, den externen Variablen Struktureingriff und Standort und der Rückkopplungsschleife Bestandestruktur $\rightarrow$ Zuwachs $\rightarrow$ Baumzustand $\rightarrow$ Bestandesstruktur (nach PRETZSCH 2001).

Modelle, bei denen das Wachstum untrennbar mit der Art der Behandlung verbunden ist, sind in jedem Falle unflexibler als einzelbaumorientierte Waldwachstumssimulatoren. Beispiele für solche Modelle sind die Ertragstafeln von Schober, die die Entwicklung eines niederdurchforsteten Bestandes wiedergeben (SCHOBER 1975) oder die Z-BaumErtragstafeln nach Klädtke (siehe KLÄDTKE 1993). Die Art des Eingriffs bestimmt hier die Wahl der Wachstumsfunktion. Bei Einzelbaumwachstumssimulatoren werden immer dieselben Funktionen zur Steuerung der Entwicklung eines Einzelbaums verwendet. 
Jedoch ändern diese Funktionen in Abhängigkeit von Konkurrenz- und Umgebungsvariablen (siehe Kapitel 2.3.1) ihren Verlauf.

Die Vielseitigkeit des Einzelbaumwachstumssimulators ist lediglich von der Zahl der integrierten Behandlungsmodelle abhängig und somit gut geeignet, um unterschiedlichste Waldwachstumskonzepte abzubilden.

\section{Simulationszeitraum}

Die zur Fortschreibung verwendeten Gleichungen beziehen sich in der Regel auf Betrachtungsintervalle von fünf oder zehn Jahren, da die Datengrundlage der Einzelfunktionen meist aus Wiederholungsmessungen von Versuchsflächen stammt, die mit diesen Zeitabständen aufgenommen werden. Ist eine feinere Aufgliederung der Ergebnisse gefragt, so können sie lediglich interpoliert werden. Reiht man mehrere Simulationsintervalle aneinander, so sind einzelbaumbasierte Wachstumssimulatoren in der Lage, Fortschreibungen bis zu dreißig oder vierzig Jahren durchzuführen. Bei wesentlich längeren Zeiträumen sind die Aussagen mit zu hohen Prognosefehlern behaftet.

Die Analyse der Auswirkungen von wirtschaftlichen Maßnahmen ist besonders interessant, wenn die Simulation mindestens einen Zeitrahmen widerspiegelt, für den betriebswirtschaftliche Entscheidungen getroffen werden müssen. Mittels eines Zeitraums von 30-40 Jahren können zwar keine Ergebnisse produziert werden, die Aussagen über den gesamten Produktionszeitraum von Wertholz erlauben, jedoch ist eine über den üblichen mittelfristigen Zeithorizont von zehn Jahren hinausgehende Planungsunterstützung möglich.

\section{Darstellung multifunktionaler Kriterien}

Es kommt immer häufiger vor, daß die Öffentlichkeit oder verschiedene Interessengruppen in Entscheidungen, die den Wald betreffen, eingebunden werden (VON TEUfFEL ET AL. 2006). Da die Entscheidungen unter Berücksichtigung einer Vielzahl von Waldfunktionen getroffen werden sollen, ist die Entscheidungsfindung komplex.

Einzelbaumbasierte Modelle bieten eine höhere Auflösung als Bestandes- oder Verteilungsmodelle. Durch Aggregation der Einzelbaumwerte sind aber auch gröbere Informationen abrufbar. Durch den Einsatz eines einzelbaumbasierten Simulators können also Auswirkungen auf Einzelbäume, Teilkollektive, Bestände oder Strukturen untersucht werden. Da die zugrundeliegende Beschreibungsebene identisch mit der Ebene der biologischen Anschauung ist, sind die Modellergebnisse für den Anwender besonders leicht zugänglich.

Damit auch Nicht-Experten mit in den Entscheidungsprozeß eingebunden werden können, ist eine Visualisierung der Auswirkungen unabdingbar. Die graphischen Benutzeroberflächen der Waldwachstumssimulatoren auf Einzelbaumbasis werden laufend um geeignete Komponenten ergänzt. 


\subsection{Abbildung verschiedener Bewirtschaftungsformen in Einzelbaumwachstumsmodellen}

Um einen Simulator als forstliches Managementinstrument verwenden zu können, ist die Implementierung verschiedener waldbaulich steuernder Maßnahmen unabdingbar (siehe auch Hasenauer 2004 oder Söderberg und Ledermann 2003). Da Einzelbaumwachstumsmodelle beste Voraussetzungen bieten, die Auswirkungen forstlicher Eingriffe transparent darzustellen (siehe Kapitel 2.3.2, Abschnitt „Abbildung forstlicher Eingriffe"), werden hier unterschiedliche Modellansätze vorgestellt.

In Einzelbaumwachstumsmodellen können lediglich Eingriffe abgebildet werden, die sich auf Bäume ab dem Stangenholzalter beziehen, da Einzelbäume in dieser Art von Modellen erst ab einer bestimmten Durchmesser- oder Höhengrenze abgebildet werden. Die Ursache dafür liegt darin, daß die grundlegenden Funktionen dieser Modelle mit Hilfe von Daten entwickelt wurden, die sich auf Bäume beziehen, die dem Verjüngungsalter entwachsen sind. Als Grenze zur Verjüngung wird zum Beispiel in Langzeitplots häufig die Höhe von 1,30m angesehen; Inventuren verwenden oft einen gewissen Brusthöhendurchmesser von zum Beispiel 5,5cm oder $7 \mathrm{~cm}$ als Aufnahmekriterium (siehe auch Niedersächsische Landesforstverwaltung 1987 oder FBVA 1994). Für die Entwicklung der Bäume unterhalb dieser Grenzen werden in Einzelbaumwachstumsmodellen getrennte Verjüngungsmodelle verwendet. Es gibt einerseits Modelle, die die gesamte Entwicklung junger Bäume vom keimenden Samen bis zum Übergang in den Bestand, modellieren. Andererseits findet man Modelle vor, die lediglich die Wahrscheinlichkeit des Einwuchses von Bäumen der Verjüngung in den Bestand prognostizieren. Diese „Einwuchsmodelle“ (siehe zum Beispiel LeDERMAnN 2002) schätzen die Anzahl und Art der neuen Bestandesmitglieder in Abhängigkeit von Bestandesparametern. In beiden Fällen werden lediglich Verteilungsfunktionen oder Wahrscheinlichkeiten verwendet, in denen Läuterungs- und Jungwuchspflegeeingriffe derzeit nicht berücksichtigt sind.

Im Verlauf des weiteren Bestandeslebens werden Wertästungs-, Durchforstungs- und Endnutzungseingriffe durchgeführt. Bislang stellen die gängigen Simulatoren jedoch nur Durchforstungs- und Endnutzungs-, aber noch keine Ästungsalgorithmen zur Verfügung. Einen Überblick über verschiedene Durchforstungs- und Endnutzungsalgorithmen ist in der Veröffentlichung von Söderberg und Ledermann (SödeRberg UND LedeRmann 2003) zu finden. Die dort vorgestellten Modelle unterscheiden sich darin, ob sie aus einer Datenbasis entwickelt wurden, positionsabhängige Entscheidungen treffen und wie sie in den Simulator eingebunden werden. Die Veröffentlichung ordnet die Modelle drei verschiedenen Klassifikationspaaren zu:

- Empirische Algorithmen imitieren den Entscheidungsprozeß oder modellieren die Auswirkungen eines ausgeführten Eingriffs. Sie werden im Gegensatz zu analytischen Algorithmen aus empirischen Durchforstungsdaten entwickelt. Analytische Algorithmen entscheiden über die Entnahme von Bäumen, indem sie eine Durchforstungs- oder Endnutzungstheorie imitieren. 
- Distanzabhängige Algorithmen hängen von Nachbarschaftsbeziehungen der Bäume ab. Bei verändertem Bestandesaufbau und sonst identischen Startbedingungen führen sie, im Gegensatz zu distanzunabhängigen Algorithmen, zu neuen Ergebnissen.

- Deterministische Algorithmen verwenden in Abgrenzung zu stochastischen keine Zufallseffekte bei der Entscheidung über zu entnehmende Bäume. Sie wählen also in einer gleichbleibenden Ausgangssituation stets die gleichen Individuen aus. Auch eine Kombination aus beiden Typen ist denkbar, wenn eine Gruppe von Bäumen durch feste Regeln ausgewählt, die Entscheidung über den Einzelbaum jedoch zufällig getroffen wird.

Ein Modell kann mehreren Klassifikationen gleichzeitig zugeordnet werden. Zum Beispiel kann man sich ein empirisches, distanzabhängiges und deterministisches Modell im Gegensatz zu einem analytischen, distanzunabhängigen und deterministischen Modell vorstellen. Das Vorliegen eines empirischen schließt jedoch einen analytischen Algorithmus, Distanzabhängigkeit Distanzunabhängigkeit aus.

Wie im Kapitel 2.2.3 unter dem Abschnitt "Durchforstungen“ beschrieben (siehe auch Assmann 1961, S. 208-214), unterscheiden sich die verschiedenen Eingriffe in ihrer

- Intensität

- Durchforstungsstärke

- und Art

Eingriffsmodelle können im Rahmen eines Einzelbaumwachtumsmodells die Intensität simulationsbedingt immer nur in den gleichen Intervallen anpassen, die bei der Modellierung des Wachstums zugrunde liegen. Das Durchforstungsintervall kann also immer nur ein Vielfaches des verwendeten Wachstumsintervalls darstellen. Die Durchforstungsstärke wird je nach verwendetem Modell über absolute Grenzwerte oder über den entnommenen Prozentsatz anhand des entnommenen oder verbliebenen Volumens, der Grundfläche oder der Stammzahl gesteuert. Um die verschiedenen Eingriffsarten abzubilden, ist man auf detaillierte Beschreibungen der Entscheidungskriterien oder der Durchforstungstheorie angewiesen. Meist existieren allerdings nur verbale Beschreibungen, so daß die Übersetzung in klare Regelanweisungen für ein Computermodell schwerfällt. Selbst detaillierte verbale Definitionen, wie sie zum Beispiel in den Arbeitsplänen zur Anlage forstlicher Versuchsanlagen gegeben werden (siehe AssmanN 1961, ab S. 205), können von verschiedenen Anwendern unterschiedlich interpretiert werden. Gerade in ungleichaltrigen Mischbeständen werden häufig differierende Entscheidungen getroffen. Eine multikriterielle Entscheidung erschwert eine Standardisierung weiter. So kann zum Beispiel die Bedeutung von Qualitätspflege, Biodiversitätserhaltung oder Massenaufbau unterschiedlich gewertet werden. Am einfachsten gestaltet sich noch Auswahl und Wertung von Kriterien in gleichaltrigen Reinbeständen, die zur Massenproduktion verwendet werden. 
Die folgenden Unterkapitel bieten einen Überblick über verschiedene Ansätze europäischer Waldwachstumssimulatoren ${ }^{10}$. Es wird beschrieben, welche Durchforstungsund Endnutzungsarten die Simulatoren in der Lage sind abzubilden. Da die Simulatoren ständiger Weiterentwicklung unterliegen, erhebt die Beschreibung keinen Anspruch auf Vollständigkeit. Es werden automatisierte, aber keine manuellen Verfahren betrachtet. Manuelle Auswahlverfahren sind für eine Simulation auf Betriebs- oder Landschaftsebene, wie sie in dieser Arbeit vorgestellt wird, nicht geeignet. Auch die Kategorie der Endnutzung bei Kalamitäten wurde nicht berücksichtigt, da die Prognose und der Umgang mit Kalamitäten nicht im Rahmen dieser Arbeit behandelt werden.

\subsubsection{Hoch- und Niederdurchforstungen}

\section{Stammzahlen-, Volumen- und Grundflächensteuerung}

Ein rein analytischer Ansatz zur Abbildung von Hoch- und Niederdurchforstungen wird verwendet, indem lediglich die Auswirkungen eines Durchforstungseingriffs auf die Stammzahl, die Grundflächenentwicklung oder die Durchmesserverteilung abgebildet werden. Dabei macht es für die Umsetzung eines Modells zunächst keinen Unterschied, ob es sich bei den Vorgaben für Stammzahlen, Volumina oder Grundflächen um Werte aus Hoch- oder Niederdurchforstungen handelt.

Ein Beispiel für die Umsetzung eines solchen Ansatzes ist im Wachstumssimulator SILVA zu finden. Dort kann eine Bestandesentwicklung bei Niederdurchforstungen gemäß den Ertragstafeln von Assmann und Franz 1963/ Assmann und Franz 1965 nachgeahmt werden. Wenn die in den Ertragstafeln angegebene Grundfläche geringer ist als die tatsächlich vorgefundene, wird die letztgenannte auf das Niveau der Ertragstafel abgesenkt.

Flexibler ist der unter PROGNAUS angebotene Ansatz, bei dem der Benutzer entweder das angestrebte Volumen, die angestrebte Stammzahl oder die angestrebte Grundfläche pro Hektar für fünf Durchmesserklassen unterschiedlich und frei definieren kann.

Ein weiterer grundflächenbasierter Ansatz wird bei BWINPro vorgestellt. Dort werden die tatsächlichen Grundflächen reduziert (nach DöbbeleR und SpelLmann 2002), wenn sie über einem gewissen prozentualen Anteil der bei maximaler Dichte erreichbaren Grundfläche (nach Sterba 1975, Sterba 1981 und Sterba 1987) liegen. In der speziell für Sachsen entwickelten Version BWINPro-S können klassische Hoch- und Niederdurchforstungsvarianten durch die Anwendung von Grundflächenleitkurven nach Wenk (Wenk, Antanaitis und Smelko 1990) durchgeführt werden (siehe Schröder, Röhle UND MÜNDER 2005).

Die Umsetzung anderer (zum Beispiel unter Kapitel 2.2.3 im Abschnitt „Durchforstungen“) genannter Modelle ist ohne weiteres denkbar. Alle Algorithmen dieser Art müssen jedoch noch mit Auswahlroutinen verbunden werden, um den vorgesehenen Eingriff umzusetzen.

10 Gemeint sind die Modelle MOSES (siehe zum Beispiel Hasenauer 1994), SILVA 2.2 (siehe zum Beispiel Pretzsch und Kahn 1995, Pretzsch 2001 und Seifert et al. 2005), STAND (Pukkala und Mina 1997), BWINPro (siehe zum Beispiel Nagel 2002A und NAgel 2005) und PROGNAUS (siehe zum Beispiel Monserud und Sterba 1996 und Sterba und Monserud 1996). 
In BWINPro wird dieses zum Beispiel durch eine zufällige Auswahl aus Teilkollektiven umgesetzt, die sich an der relativen Baumhöhe orientieren. Neben der Ziehung von Zufallszahlen ist auch die Betrachtung von Einzelbaumkriterien denkbar. Diese Kriterien müßten sich dann aber für Nieder- und Hochdurchforstung unterscheiden. Bislang gibt es für eine solche Vorgehensweise keine Beispiele.

\section{Fuzzy-Logik}

Durch die auf Fuzzy-Logik basierende Modellierung waldbaulicher Eingriffe soll menschliches Verhalten beim Treffen von Durchforstungsentscheidungen nachgebildet werden (KAHN 1995 a und KAHN 1995 B). Somit gehört diese Art der Modelle in die Gruppe der empirischen Ansätze.

Um ein Fuzzy-Logik-Modell zu entwickeln, werden verbale Durchforstungsvorschriften, wie sie zum Beispiel in den Vorschriften des Vereins forstlicher Versuchsanstalten 1902 formuliert wurden (siehe Assmann 1961, ab S. 205), als Grundlage verwendet. Bei der Umsetzung der Angaben werden fünf bis sieben verbale „WENN ... UND ... DANN ...“ Regeln aufgestellt. Die Abbildungsmöglichkeiten hängen dabei wesentlich von verfügbaren Parametern ab. Als Eingabe werden Baumparameter benötigt, die Ausgabe definiert sich als Durchforstungsdringlichkeit. Kahn zum Beispiel verwendet als Eingabevariablen die relative Baumstärke und den Konkurrenzdruck. Die relative Baumstärke definiert sich in seinem Modell über den relativen Baumdurchmesser und die relative Summenhäufigkeit aus der Durchmesserverteilung. Den Konkurrenzdruck bildet der Konkurrenzindex KKL (Kronenkonkurrenz um Licht) ab (siehe PRETZSCH 1994).

Gemäß dem Konzept der unscharfen Mengen (ZimmermanN 1991) werden den Eingangsparametern in einem ersten Schritt linguistische Variablen wie „hoch“, „mittel“ oder „niedrig“ zugeordnet. So können im zweiten Schritt unscharfe Entscheidungsvorschriften, wie zum Beispiel „WENN Baumstärke gering UND Konkurrenzdruck hoch, DANN Durchforstungswahrscheinlichkeit hoch" abgebildet werden. In einem dritten Schritt wird die unscharfe Ausgabe defuzzifiziert, indem das sogenannte Flächenschwerpunktverfahren angewendet wird. Die Bäume können im Anschluß in einem vierten Schritt nach ihren Durchforstungsdringlichkeiten sortiert werden. In der eigentlichen Entnahme (Schritt fünf) werden entweder alle Bäume entfernt, denen eine Durchforstungsdringlichkeit zugeordnet wurde, oder eine Volumen-, Grundflächen- oder Stammzahlgrenze begrenzt die Entnahmemenge. Durch die Entnahme einzelner Bäume kann sich die Durchforstungsdringlichkeit der verbliebenen Individuen ändern. Daher werden nach Entnahme einiger Bäume die Durchforstungsdringlichkeiten eines Teils des Kollektivs neu berechnet.

Bislang wurden Fuzzy-Logik-basierte Modelle für Hoch- und Niederdurchforstungen kalibriert. Tatsächliche Entscheidungen konnten durch Anpassung der Regeln mit diesen Modellen gut abgebildet werden. Schwerer fällt es, wenig untersuchte Durchforstungsvarianten abzubilden, da keine Daten für eine solche Justierung vorliegen. 


\subsubsection{Selektive Z-Baum-Förderung und Entnahme}

Selektive Durchforstungsalgorithmen (siehe auch SöDERBERg UND LeDERMANN 2003) sind in der Lage, Hochdurchforstungen zur Vorbereitung von Zielstärkennutzungen abzubilden. ZBäume können nach verschiedenen Gesichtspunkten ausgewählt und unterschiedlich stark freigestellt werden. Die Routinen sind an die Auswahlkriterien angepaßt, die bei der Wertholzproduktion im Vordergrund stehen. In den meisten Modellen wird zwischen der Auswahl der zu fördernden und der Entnahme der bedrängenden Bäume unterschieden.

\section{Z-Baum-Auswahl}

Die Simulatoren SILVA, MOSES und BWINPro sind Beispiele für einen getrennt stehenden Auswahlalgorithmus. Als Auswahlkriterien gelten dort Vitalität, Qualität, relative Dimension, Baumart und der Abstand der zu fördernden Bäume. Durch welche Merkmale die Erfüllung dieser Auswahlkriterien überprüft wird, hängt von dem jeweiligen Modell ab. Zum Beispiel ist es einerseits möglich, die Stammqualität anhand des HD-Verhältnisses (Höhen/ Durchmesser-Verhältnis) in Kombination mit der Kronenlänge zu messen. Andererseits können auch Astigkeitsparameter, die zusammen mit Stammformfunktionen und dem Verhältnis Baumhöhe/ Kronenansatz ausgewertet werden (nach dem Modell von SCHMIDT 2001), die Qualität definieren.

\section{Entnahme der Bedränger}

Einzelbaummodelle bieten einerseits die Möglichkeit, durch einen analytischen Ansatz die Durchforstungstheorie abzubilden (zum Beispiel durch die Ermittlung der Wahrscheinlichkeit, mit der ein Baum entfernt wird). Andererseits kann auch die Entscheidung über den zu entnehmenden Baum anhand von Heuristiken festgemacht werden (siehe DAUME UND ROBERTSON 2000), die die einzelnen Entscheidungsschritte eines Försters nachbilden (empirischer Ansatz). Das Modell ThiCon (siehe NAgel 1996, Albert 2002 und Albert und Guericke 2003) ist ein Beispiel für ein solches empirisches Modell. Die Entscheidungen können zum Beispiel Baumposition, Baumdurchmesser, Art, Vitalität, Qualität, Kronenspannung oder Abstand zwischen den betrachteten Bäumen mit einbeziehen (siehe Albert 2002).

Unabhängig davon, ob empirische oder analytische Funktionen verwendet werden, kann die Entnahme von Bedrängern in drei Schritte unterteilt werden:

- Die Fokussierung auf einen bestimmten Teil des Bestandes (zum Beispiel Umgebung eines Z-Baums bis hin zur Ausdehnung, die dem dreifachen Kronenradius des Z-Baums entspricht).

- Identifizierung der Bedränger (zum Beispiel durch die Betrachtung der Kronenüberlappung).

- Geregelte Entnahme von Bedrängern (Festlegung der Reihenfolge und der Menge). 
In der Regel werden in Simulationen die Umgebungen aller Z-Bäume nacheinander auf potentielle Bedränger hin untersucht. Als Maß für die Konkurrenz zwischen zwei Bäumen ist neben der Kronenüberlappung auch der A-Wert nach Johann (siehe JoHANN 1982 und JoHANn 1983) geeignet. Er setzt sich aus der Höhe des Z-Baums (Höhe z $_{\text {) }}$, dem Abstand des betrachteten Nachbarn zum Z-Baum (Abstand $\mathrm{bz}_{\mathrm{bz}}$ ) und den Brusthöhendurchmessern von Z-Baum $\left(\mathrm{BHD}_{\mathrm{z}}\right)$ und betrachtetem Nachbarn $\left(\mathrm{BHD}_{\mathrm{b}}\right)$ zusammen:

$$
A-\text { Wert }=\frac{\text { Höhe }_{z}}{\text { Abstand }_{b z}} \cdot \frac{B H D_{b}}{B H D_{z}}
$$

Nachdem die vorliegende Konkurrenzsituation anhand eines geeigneten Index festgehalten wurde, werden zu entnehmende Bedränger identifiziert. Den Freistellungsgrad eines Z-Baums kann man durch das Festlegen einer Grenze steuern, ab der ein benachbarter Baum als Bedränger definiert wird. Zum Beispiel gelten dann alle Bäume mit einem A-Wert, der eine festgelegte Grenze überschreitet als Bedränger. Erlaubt das gewählte Maß (wie der A-Wert oder ein Kronenüberlappungsprozent) eine Sortierung nach dem Grad der Bedrängung, kann man, beim stärksten Bedränger beginnend, eine festgelegte Anzahl an Bäumen pro Z-Baum entnehmen. Eine weitere Möglichkeit, die Freistellung der zu fördernden Bäume zu beschränken, ist die Festlegung eines gewissen Radius, der um einen Z-Baum herumgelegt wird und aus dem die bedrängenden Individuen entfernt werden (siehe auch SöDERBERG UND LEDERMANN 2003).

\section{Kombinierter Z-Baum-Auswahl- und Freistellungsalgorithmus}

Einen von den bislang vorgestellten Modellen abweichenden analytischen Algorithmus bietet der Simulator PROGNAUS (siehe Söderberg UND Ledermann 2003). In diesem Modell ist die Auswahl von Z-Bäumen mit der Wahl zu entnehmender Individuen eng gekoppelt. Anhand von LOGIT-Funktionen werden Wahrscheinlichkeiten errechnet, mit denen Z-Bäume und zu entnehmende Bäume einer Baumliste ausgewählt werden. Dabei wird die relative Dimension des Baums, die Stabilität, die Stammqualität, Stammverletzungen und die durch den Baum repräsentierte Baumzahl pro Hektar berücksichtigt. Derzeit ist dieser Algorithmus nur für die Baumart „Fichte“ in der Literatur vorgestellt worden.

\subsubsection{Zielstärkennutzung}

In allen fünf einzelbaumbasierten Waldwachstumssimulatoren (SILVA, MOSES, PROGNAUS, BWINPro) können Zielstärkennutzungen modelliert werden. Eine Voraussetzung für den Einsatz solcher Algorithmen ist die Festlegung baumartenspezifischer Zielstärken. Je nach Modell können nun alle Bäume bei Erreichen der Zielstärke entnommen werden oder eine eingeschränkte Entnahme implementiert sein. Sollen zum Beispiel nur 20\% der möglichen Bäume entnommen werden, wird jeder zielstarke Baum nur mit einer Wahrscheinlichkeit von 20\% entfernt (siehe PRETZSCH 2001). Repräsentieren die Bäume in der Simulation durch einen Faktor das Vielfache eines Baums des Bestandes, so kann dieser Faktor bei einer teilweisen Ernte um das zu 
erntende Prozent reduziert werden. Ein solches Vorgehen ist in PROGNAUS umgesetzt (siehe Söderberg Und Ledermann 2003).

\subsubsection{Schlagweise Nutzung}

Der bestandesweise Kahlschlag an sich ist in einem einzelbaumbasierten Wachstumssimulator leicht umzusetzen, indem bei Erreichen einer gewissen Alters- oder Durchmessergrenze alle Bäume entnommen werden. Gerade im Zusammenhang mit der Nachbildung von Saum- oder Femelschlägen kann es überdies sinnvoll sein, die Endnutzungsaktivitäten auf einzelne Streifen, Rechtecke oder Kreise beschränken zu können. Generell ist in positionsabhängigen Modellen jede geometrische Beschränkung der Eingriffsaktivität denkbar und auch leicht umsetzbar. Innerhalb der jeweils gewählten geometrischen Form können alle Bäume oder nur Anteile des Bestandes entnommen werden. Theoretisch läßt sich jede geometrische Einschränkung mit jedem Eingriffsalgorithmus verbinden. Der Simulator BWINPro-S ist zum Beispiel in der Lage, Schirmschlag, Kahlschlag, Räumungshiebe, Lochhiebe und Saumhiebe umzusetzen (siehe ScHRÖdER, RöHLE UND Münder 2005). SILVA und MOSES ermöglichen streifenweise Entnahmen und Entnahmen innerhalb eines definierten Rechtecks. Für eine eingeschränkte Auswahl können in diesen Simulatoren simple Algorithmen verwendet werden, so daß zum Beispiel nur jeder dritte Baum (bei streifenweiser Nutzung) oder nur die Bäume einer bestimmten Durchmesserklasse (bei der Auswahl eines Rechtecks) herausgefiltert werden.

\subsubsection{Erhaltung eines Plenterwaldgefüges}

In Analogie zu dem Ansatz, Hoch- und Niederdurchforstungen durch Stammzahlen-, Volumen- oder Grundflächensteuerung abzubilden, besteht auch die Möglichkeit, einen Bestand gemäß eines Plentergefüges zu bewirtschaften. Dazu wird die aktuelle Besetzung der Durchmesserklassen der Gleichgewichtskurve eines Plenterwaldes angepaßt, indem Bäume aus überbesetzten Durchmesserklassen entnommen werden. Ein solches Modell ist im Simulator SILVA zu finden (siehe SöDERBERg Und Ledermann 2003). Die Entscheidung darüber, welcher konkrete Baum bei der Entnahme entfernt wird, ist in diesem Simulator zufallsgesteuert. Sinnvoll ist der Einsatz dieses Modells nur, wenn die tatsächliche nicht zu weit von der angestrebten Bestandesstruktur abweicht. Den Umbau von gleichaltrigen Reinbeständen in einen Plenterwald kann der Simulator demnach nicht abbilden.

\subsubsection{Sonstige Ansätze zur Abbildung forstlicher Eingriffe}

\section{Weiterführung der bisherigen waldbaulichen Behandlung}

Thomas Ledermann entwickelte ein Modell, mit dem er die bisherige Behandlung eines Waldes nachbildet (Ledermann 2002 A). Dazu verwendet er logistische Funktionen, die einerseits die Wahrscheinlichkeit prognostizieren, daß ein Eingriff in einer Probefläche stattfindet und andererseits voraussagt, ob ein bestimmter Baum dieser Stichprobe entnommen wird. Durch die Entwicklung eines solchen Modells kann man zwar feststellen, 
ob eine Wirtschaftsweise zielführend ist und welche Resultate leichte Änderungen dieses Konzepts zur Folge haben. Es ist aber nicht in der Lage, völlig neue Strategien abzubilden.

\section{Optimierende Modelle}

Optimierende Modelle bilden keine Durchforstungsregeln oder Durchforstungstheorien ab. Vielmehr zeigen sie, welche Entscheidungen getroffen werden müßten, um ein gewisses Set an Zielen verwirklichen zu können. Ein Beispiel ist das Modell aus dem Simulator STAND, das von Pukkala und Miina beschrieben wird (PukKala Und MıINA 1997). Da in optimierenden Modellen die Art der Durchforstung nicht im vorhinein festgelegt werden kann, entfällt dieser Ansatz für den Vergleich forstlicher Managementstrategien. Aus dem Durchforstungsergebnis kann auf keine generelle Empfehlung für eine Art der Bewirtschaftung geschlossen werden.

\section{Material und Methoden}

Die anschließenden Unterkapitel erläutern dem Leser die Umsetzung von vier beispielhaften Entwicklungsszenarien. Zunächst werden Ziele und Inhalte der untersuchten Waldbaustrategien erläutert (siehe Kapitel 3.1). Diese Strategien sollen in Simulationsläufen auf die in Kapitel 3.2 vorgestellten Simulationsobjekte angewendet werden. Ein Überblick über die Simulation der Szenarien und die Auswertung der Ergebnisse werden in Kapitel 3.3 vorgestellt. Auf die einzelnen Verarbeitungsschritte, die den Vergleich der forstlichen Managementstrategien ermöglichen, gehen die Kapitel 3.4, 3.5 und 3.6 ein. Sie beschreiben die Datenvorbereitung und die Ausgabe des Status Quo, die szenarienabhängige Fortschreibung und Ausgabe der Simulationsergebnisse sowie die Auswertung der ausgegebenen Parameter.

\subsection{Umgesetzte Waldbaustrategiebeispiele}

Das Ziel dieser Arbeit ist es, eine Möglichkeit aufzuzeigen, forstliche Managementstrategien „Vom Schreibtisch aus“ vergleichen zu können. Der Simulationszeitraum wurde auf vierzig Jahre festgelegt. Dadurch ist eine Beurteilung der mittelfristigen Waldbauplanung möglich, und Anhaltspunkte für die langfristige Planung sind gegeben. Vorteile, Risiken und Konfliktpotentiale können schon im Vorfeld analysiert werden. Auch besteht die Chance, durch Abwandlung des geplanten Vorgehens viele Probleme erst gar nicht entstehen zu lassen.

Neben dem in den Niedersächsischen Landesforsten umgesetzten Programm LÖWE werden auch Beispiele vorgestellt, die nicht das Konzept eines konkreten Betriebs abbilden. Sie stehen exemplarisch für mögliche Strategien privater Waldbesitzer. Die Ausformulierung dieser Konzepte stützt sich einerseits auf Literatur, die Aspekte von Waldbaukonzepten beleuchtet, und andererseits auf mündlich bestätigte Erfahrungswerte privater Waldbesitzer. Die gewählten Varianten sollen dazu dienen, einen Eindruck der Variationsbreite möglicher Waldbaukonzepte zu geben. Das Spektrum reicht von einer Ökonomie und Ökologie vereinenden Alternative (LÖWE), der unbedingten Umsetzung 
naturnaher Bestockung (PNV) über eine ertragsorientierte Wahlmöglichkeit (ERTRAG) bis hin zu einer kaum steuernd eingreifenden Variante (PROZESS).

\subsubsection{LÖWE}

Mit dem Konzept LÖWE (Ľangfristige ökologische Waldentwicklung, in NIEDERSÄCHSISCHE LANDESFORSTVERWALTUNG 1991) verfolgt die Niedersächsische Landesregierung das Ziel, den Konflikt zwischen Ökonomie und Ökologie im Landeswald auszugleichen. Diesem Beispiel folgend haben sich in den zurückliegenden Jahren die waldbaulichen Ziele vieler Landesforstverwaltungen geändert. Für Privatwaldbesitzer ist das Konzept in Niedersachsen zwar nicht verbindlich, bietet innen aber Anregungen (JANSSEN 1992), die zunehmend aufgegriffen werden. Zum Beispiel wird die Mischwaldvermehrung vielerorts in allen Besitzarten angestrebt (MüLLER 1994).

Die 1991 erklärten Ziele des Konzepts LÖWE sind Gemeinnützigkeit, Nachhaltigkeit und Wirtschaftlichkeit. Durch die Formulierung der Gleichrangigkeit von Nutz-, Schutz- und Erholungsfunktion des Waldes soll der höchstmögliche Nutzen für die Allgemeinheit geschaffen werden. Ein stabiler Waldaufbau, naturnahe Waldbautechniken und die Verwirklichung des multifunktionalen Ansatzes werden auf ganzer Fläche angestrebt (Siehe auch Отто 1989 und Отто 1991). Das Konzept stimmt damit weitestgehend mit den wichtigsten waldbaulichen Zielsetzungen und Bewirtschaftungsgrundsätzen der Naturgemäßen Waldwirtschaft überein, die vom ANW gefordert werden. Im Einzelnen bestehen folgende 13 Grundsätze als die Leitlinie waldbaulichen Handelns:

I. Grundsatz: Bodenschutz und standortgemäße Baumartenwahl

II. Grundsatz: Laubwald- und Mischwaldvermehrung

III. Grundsatz: Ökologische Zuträglichkeit

IV. Grundsatz: Bevorzugung natürlicher Waldverjüngung

V. Grundsatz: Verbesserung des Waldgefüges

VI. Grundsatz: Zielstärkennutzung

VII. Grundsatz: Erhaltung alter Bäume, Schutz seltener und bedrohter Pflanzen- und Tierarten

VIII.Grundsatz: Aufbau eines Netzes von Waldschutzgebieten

IX. Grundsatz: Gewährleistung besonderer Waldfunktionen

X. Grundsatz: Waldrandgestaltung und -pflege

XI. Grundsatz: Ökologischer Waldschutz

XII. Grundsatz: Ökosystemverträgliche Wildbewirtschaftung

XIII.Grundsatz: Ökologisch verträglicher Einsatz der Forsttechnik 
Die Erholungsfunktion des Waldes wird durch diese Grundsätze nicht eingeschränkt. Das LÖWE-Programm wird in Einklang mit fachübergreifenden Programmen (wie dem Landesraumordnungsprogramm und dem Regionalen Raumordnungsprogramm) umgesetzt und bietet den Erholungswald als Waldfunktion (siehe NiEDERSÄCHSISCHE LANDESFORSTVERWALTUNG 1991).

Auf die Schutzfunktion des Waldes gehen besonders die Grundsätze I (Bodenschutz und standortgemäße Baumartenwahl), VII (Erhaltung alter Bäume, Schutz seltener und bedrohter Pflanzen- und Tierarten), VIII (Aufbau eines Netzes von Waldschutzgebieten), IX (Gewährleistung besonderer Waldfunktionen) und X (Waldrandgestaltung und -pflege) ein. Aber auch die Bewirtschaftungsart orientiert sich stets an ökologischer Verträglichkeit. Das zeigen die Grundsätze XI (Ökologischer Waldschutz), XII (Ökosystemverträgliche Wildbewirtschaftung) und XIII (Ökologisch verträglicher Einsatz der Forsttechnik).

Die Nutzfunktion des Waldes wird besonders durch die Kombination der Grundsätze II (Laubwald- und Mischwaldvermehrung), IV (Bevorzugung natürlicher Waldverjüngung), V (Verbesserung des Waldgefüges) und VI (Zielstärkennutzung) beeinflußt. Betriebswirtschaftlich betrachtet führt die Anwendung dieser Grundsätze zur Ausprägung der folgenden vier Erfolgsfaktoren eines LÖWE-Waldes (siehe auch MERKER 1997 und MERKER 1998):

\section{Flexibilisierung von Produktion und Absatz}

Diversität im Wald trägt zur Verminderung des Marktrisikos bei (siehe auch Отто 1989). Ein strukturreicher Wald aus Misch- und Reinbeständen macht das Angebot verschiedener Holzarten, die Erzeugung einer gefächerten Qualitätspalette und der Bereitstellung unterschiedlichster Sortimente möglich. Gerade durch die Zielstärkennutzung verspricht man sich flexibleres Agieren (durch Mengenanpassung und Steuerung des Sortimentangebots) auf dem Holzmarkt und die Möglichkeit, sich an die Bedürfnisse der Holzindustrie anpassen zu können (WollboRn 2004 und JANSSEN 2000).

\section{Anpassung der Produktionsintensitäten}

Kostspielige Begründungs- und Pflegeeingriffe werden auf lohnende Bestände konzentriert, indem man sich standortsangepaßte Massen- und Wertziele steckt. Auch das Unterlassen von Pflegemaßnahmen ist denkbar. Damit verbundene Risiken wirken sich in ertragsschwachen Beständen monetär relativ gering aus (MöHRING 1994). Investitionen in Bestände können reduziert werden, indem bestandesinterne Prozesse strukturreicher Wälder gefördert und genutzt werden. Bereits 1967 bringt Steinlin (STEINLIN 1967) in diesem Zusammenhang den Begriff der biologischen Automation auf, der wie folgt von Pausch (PAUSCH 2002) aufgegriffen und erweitert wird: „Biologische Automation bedeutet im Idealfall, daß die für die angestrebte Holzerzeugung notwendigen Wachstumsabläufe allein durch die Entnahme der Endprodukte („Vollzug der naturalen Ziele“) erhalten und gesteuert werden können..... In diesem Sinne verbindet zum Beispiel auch Wollborn (WolLBORN 2004) mit der Umsetzung des LÖWE-Programms Kostensenkungspotentiale.

Er nennt die Qualitätsförderung des unter Schirm erwachsenen Nachwuchses, eine Minimierung der konkurrenzsteuernden Eingriffe, die Nutzung des Lichtungszuwachses 
der Altbäume sowie eine Minimierung der Risiken für den Nachwuchs (wie Mäuse oder Spätfrost) und die Altbestände (wie Windwurf, Insektenmassenvermehrungen oder Feuer).

\section{Nutzung von Kostendegressionseffekten}

Mit der Nutzung von Kostendegressionseffekten ist laut Merker (MeRKER 1998) die Nutzung von Rationalisierungseffekten in der Flächenverwaltung und der Ernte gemeint. Durch die bestandesinterne Förderung der vertikalen Struktur und der Mischung von Baumarten sinkt gleichzeitig die Segregation der Landschaft in sichtbar erkennbare Bestände. Würden die tatsächlichen Verwaltungseinheiten (Abteilungen und Unterabteilungen) den (großflächig gesehen) gleichbestockten Einheiten angepaßt, könnten Verwaltungskosten deutlich gesenkt werden (siehe auch HöHER 1995). Werden die Erntemaßnahmen in großen Verwaltungseinheiten gebündelt und koordiniert ausgeführt, rentiert sich der Einsatz von hochtechnisierten Ernteverfahren, die einen zusätzlichen Beitrag zur Wirtschaftlichkeit leisten können (BRABÄNDER 1994).

Ein weiterer Aspekt der Kostendegression ist der geminderte Aufwand bei der Ernte von Starkholz im Vergleich zu Schwachholz, durch den die direkten Erntekosten sinken (StückMasse-Gesetz). Allerdings ist eine vermehrte Starkholzproduktion nur bei entsprechender Nachfrage sinnvoll.

\section{Vermeidung von Risiko}

Durch die Pflege der Bestandesstrukturen und durch Anbau standortgerechter Baumarten werden stabile Waldbestände gefördert, in denen das Risiko von Windwurf, Insektenmassenvermehrungen, Feuer und anderer Kalamitäten verringert ist. Da großflächige Störungen den Bestand immer wieder in pflegeintensive Altersphasen zurückversetzen, hat ein hohes Kalamitätsrisiko weitreichende wirtschaftliche Konsequenzen. Es ist in vielen Fällen betriebswirtschaftlich sinnvoller, auf Ertragssteigerung zugunsten von Risikovermeidung zu verzichten (siehe auch Отто 1989).

Bei allen wirtschaftlichen Vorteilen der Umsetzung des LÖWE-Programms müssen auch einige Nachteile bzw. Risiken genannt werden. So werden in der derzeitig notwendigen Umbauphase kostspielige Investitionen (Pflanzungen, Voranbauten, Zaunbau...) getätigt, um mehrschichtige Mischbestände mit den zuvor beschriebenen Vorteilen aufbauen zu können (siehe auch Merker 1998). Außerdem weist zum Beispiel Spellmann darauf hin, daß die Erhöhung des Laub- und Mischwaldanteils langfristig ein Absinken der mittleren Leistungsklasse der Buche, Kiefer und Fichte zur Folge haben wird, die sich auch auf den zu erwartenden Holzerlös auswirken kann.

Konkrete Handlungsanweisungen für die Umsetzung des LÖWE-Konzepts sind in den Merkblättern der Niedersächsischen Landesforstverwaltung (NIEDERSÄCHSISCHE LANDESFORSTVERWALtUng 2006) oder der Richtlinie zur Baumartenwahl (Niedersächsische LANDESFORSTVERWALTUNG 2004) nachzulesen. Diese Quellen wurden als Grundlage für die programmiertechnische Umsetzung des LÖWE-Konzepts verwendet (siehe auch Kapitel 3.5.1). 


\subsubsection{PNV}

Die Simulationsvariante PNV stellt die waldbauliche Umsetzung des gedanklichen Konstruktes der potentiellen natürlichen Vegetation $(\mathrm{pnV})$ dar, die auf eine Definition von Tüxen (TüXEN 1956) zurückgeht und durch Kowarik (KowARIK 1987) und Leuschner (LeUsCHNER 1997) erweitert wurde. Unter der pnV wird der Gleichgewichtszustand der Vegetation verstanden, der auf der Grundlage des gegenwärtigen Standortpotentials unter Berücksichtigung anthropogener Einflüsse denkbar ist (siehe Abbildung 3.1.2.a).
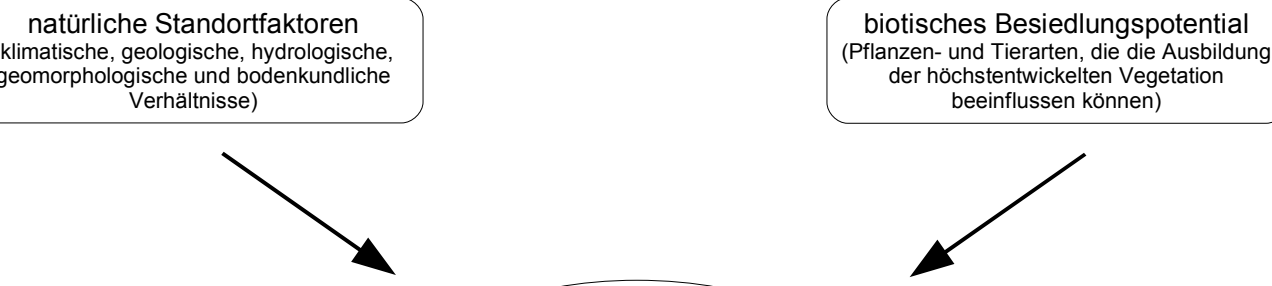
der höchstentwickelten Vegetation beeinflussen können)
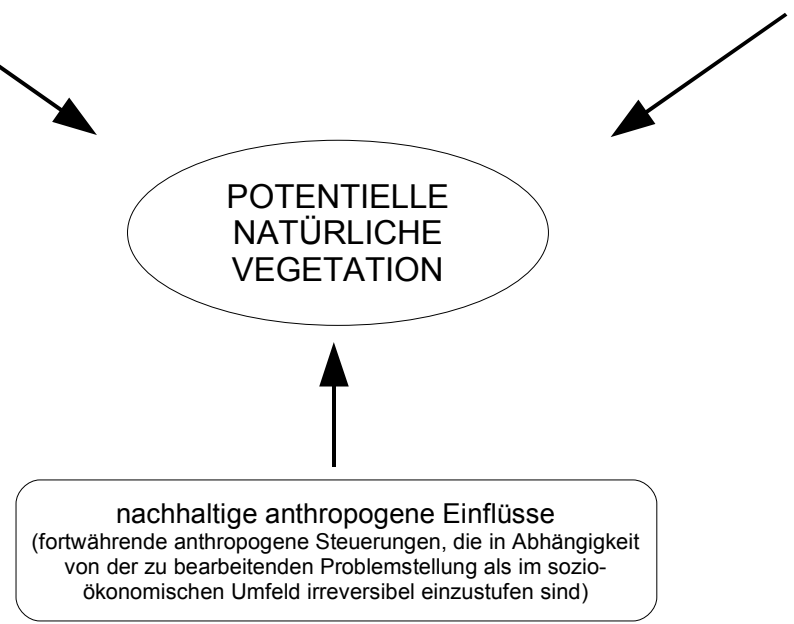

Abbildung 3.1.2.a: Parameter für die Ableitung der potentiellen natürlichen Vegetation eines bestimmten Zeitraums (aus KAISER 1996).

Diese hypothetische Vegetation berücksichtigt keine sukzessionalen Stadien, sondern geht von einem schlagartigen Einstellen des neuen Zustands aus. Das Gedankenmodell dient dazu „in stark anthropogen geprägten Landschaften eine Vorstellung von der vom Menschen unbeeinflußten Vegetation zu vermitteln“" (siehe LeUsCHNER 1997). Das pnVKonzept stellt eine in der Naturschutzpraxis, Landschaftsbewertung und -planung etablierte (siehe zum Beispiel KaISER 1999 oder PATERAK 1999) Zielvorstellung (Leitbild) dar. Z. B. dient sie als richtungsweisende Grundlage für Landschaftsprogramme, Landschaftsrahmenpläne, Landschaftspläne, Grünordnungspläne, Umwelt- oder FFHVerträglichkeitsstudien (siehe KAISER UND ZACHARIAS 2003). Auch in der Forstwirtschaft findet das Modell zum Beispiel bei der Standorts- oder Biotopkartierung Anwendung (siehe zum Beispiel Arbeitskreis Standortskartierung 2003 oder Niedersächsisches ForstPlanungsamt 1998). Als Arbeitsgrundlage dienen den verschiedenen Disziplinen Karten, die mit unterschiedlichen Kartierungsmethoden für bestimmte Gebiete in entsprechenden Maßstäben angefertigt sind. In dieser Arbeit wird auf die pnV-Karten von Kaiser und Zacharias für Niedersachsen zurückgegriffen (KAISER UND ZACHARIAS 2003). Die Karten wurden auf Basis der Bodenübersichtskarte 1:50.000 (BÜK 50) erstellt. Solche aus der BÜK 50 abgeleiteten Karten kommen unter anderem als Planungsgrundlage bei der Fortschreibung von Landschaftsrahmenplänen zum Einsatz (siehe PATERAK, BiERHALS UND PREISS 2001). Das Untersuchungsgebiet wird nach der Veröffentlichung von Kaiser und 
Zacharias auf $79 \%$ der Bestandesfläche Buchenwäldern basenarmer Standorte zugerechnet. Auf $20 \%$ der Fläche werden Eichen- und Buchenmischwälder basen- und nährstoffarmer, grundwasserferner Standorte als pnV angesehen. Der Anteil der stark wasserbeeinflußten Teile liegt unter einem Prozent. Dort kommen Typen wie StieleichenAuwaldkomplexe, Bruchwälder und sonstige Feuchtwälder bzw. Niedermoore zum Tragen.

Die Möglichkeit, die pnV als waldbauliche Zielvorstellung zu verstehen, wurde mehrfach kritisch betrachtet (siehe zum Beispiel KowariK 1987 oder ZeRBE 1997). Bei der hier vorgestellten Variante PNV steht die Anwendung der Ergebnisse als Orientierungshilfe im Vordergrund. Die Umsetzung dieses Szenarios soll nicht als alleingültige Handlungsanweisung zur Erreichung und Erhaltung der pnV mißverstanden werden. Selbst drastische Maßnahmen können allenfalls zu einer pnV-gemäßen Artenzusammensetzung beitragen (KAISER 1996). Die im Gedankenkonstrukt vorausgesetzte schlagartige Umstellung ist in der Praxis nicht möglich. Da der Begriff der pnV nicht im Zusammenhang mit einem Waldbaukonzept entwickelt wurde, werden im Szenario PNV Maßnahmen einer naturnahen Waldwirtschaft (zum Beispiel Zielstärkennutzung) angewendet, um auf programmiertechnisch greifbare Entscheidungskriterien zurückgreifen zu können (siehe auch Kapitel 3.5.2). Für diese Arbeit steht bei der Umsetzung des pnV-Konzepts die Umsteuerung der Baumartenzusammensetzung in Richtung der potentiell natürlichen Vegetation im Vordergrund. Dadurch soll die Naturnähe der Bestände erhöht werden. Erreicht wird dieses Ziel, indem die Baumarten in Durchforstungsmaßnahmen stark gefördert werden, die der pnV entsprechen. Dabei wird auf den Schutz von Minderheiten oder die Erhaltung von Habitatbäumen kein Wert gelegt. Weiterhin werden bei pnV-ferner Bestockung Pflanzungen vorgenommen.

\subsubsection{ERTRAG}

Die Variante ERTRAG setzt konsequent ökonomisch und erwerbswirtschaftlich ausgerichtete Forstwirtschaft um. Dabei stehen bei dieser Variante nicht kurzfristige Ziele, sondern die langfristige pekuniäre Nutzung im Vordergrund. Diese soll mit minimalem Aufwand und optimaler Allokation begrenzter Ressourcen nach ökonomischen Prinzipien umgesetzt werden. Dabei werden Ökologie und Ökonomie nicht als grundsätzlicher Konflikt betrachtet (siehe auch DUfFNER 2004).

In dieser Variante wird unter anderem ein Umbau der Ist-Bestockung hin zu ertragsstarken Baumarten forciert, um eine ertragsorientierte Waldbehandlung abzubilden. Es wird sowohl auf Fichten- und Douglasien- als auch auf Buchen-Reinbestände gesetzt. Ist das Potential des Standorts durch Mischbestände optimal zu nutzen, werden Fichten/ Buchenund Douglasien/ Buchenbestände angestrebt. Auf Extremstandorten (durch Nässe oder Trockenheit) werden die standörtlichen Gegebenheiten berücksichtigt, um das Kalamitätsrisiko nicht zu erhöhen. Dort lehnt sich die Baumartenwahl stark an die Variante LÖWE an. Eine gewisse Fächerung der angebotenen Produktpalette bleibt demnach durch das Verfolgen dieser Zielvorstellung erhalten, so daß flexibles Agieren auf dem Holzmarkt möglich ist. Ein Schwerpunkt wird jedoch auf die Produktion von Massensortimenten durch den Einsatz ertragsstarker Arten gelegt. Dabei spielen die 
durchsetzungsstarken Baumarten Douglasie und Fichte eine entscheidende Rolle. Auf eine künstliche Beimischung oder Erhaltung von Misch- oder Begleitbaumarten wird kein Wert gelegt.

Die Durchforstungsintervalle sind größer als in den zuvor vorgestellten Varianten, und auch die Durchforstungsstärke ist erhöht. Gleichzeitig erfolgt der erste Eingriff in einem Bestand früher. Durch dieses Vorgehen fallen größere Nutzungsmassen zum gleichen Zeitpunkt an. So können Durchforstungen effizient gestaltet werden, da höhere Entnahmevolumina automatisierte Erntemethoden unterstützen.

Auch in der Endnutzung werden höhere, mechanisierungsfreundliche Endnutzungsmassen zugelassen. Innerhalb eines baumartenabhängigen Zeitraums wird die erntereife Bestandesschicht vollständig genutzt. Der zehn bis dreißig Jahre dauernde Endnutzungszeitraum beginnt, sobald der mittlere Durchmesser der führenden Schicht einen baumartenspezifischen Ziel-Dg erreicht hat. Dieser mittlere Zieldurchmesser liegt unter den Werten, die in den Varianten LÖWE, PNV und PROZESS die Zielstärke für Einzelbäume festlegen. Im Vergleich zu den anderen drei Varianten verkürzen sich dadurch die Umtriebszeiten, weil die Bestände früher in die Endnutzungsphase kommen.

Die Wälder entwickeln sich bei dieser Variante zu weniger stark geschichteten Beständen, in denen Minderheiten oder Habitatbäume nicht geschützt werden. Reinbestände haben einen wesentlich höheren Anteil als zum Beispiel in der Variante LÖWE. Die Rahmenwerte wie Zieldurchmesser, Eingriffsintervalle oder Nutzungsmassen wurden in Anlehnung an die im wirtschaftlichen Privatwald dieser Region üblichen Werte gutachterlich festgesetzt, da keine schriftlichen Quellen für solche Erfahrungswerte vorliegen.

\subsubsection{PROZESS}

Die Idee des Prozeßschutzes ist ein relativ neues Gebiet des Naturschutzes. Remmert wirft 1988 den Gedanken auf, daß Schutzgebiete, die natürliche Prozesse ermöglichen, eine Schlüsselrolle für die Verwirklichung von Naturschutzzielen einnehmen können (REMMERT 1988). Scherzinger war einer der ersten, der die Idee für den Nationalparkgedanken konkretisierte (siehe SCHERZINGER 1990). Sturm und Fähser veröffentlichten in den darauffolgenden Jahren die Grundlagen des Prozeßschutzansatzes für Forstbetriebe (siehe Sturm 1993, Sturm 1994, Sturm 1995 a, FäHSER 1995 und Sturm 1995 в). Besonders die für Greenpeace angefertigte Studie (STURM 1994) gab wichtige Impulse für die Entwicklung der Naturland-Zertifizierungs-Richtlinie, die neben Greenpeace und Naturland auch vom BUND und Robin Wood unterstützt wird (siehe Naturland et al. 1996 oder Anonymus 1996). Der Naturschutz sieht das Prozeßschutzkonzept heute als „sinnvolle berechtigte Leitlinie <...> unter mehreren“ (siehe PIECHOCKI ET AL. 2004).

Das Prozeßschutzkonzept nach Sturm verfolgt die Zielsetzung, die „...Dynamik des Ökosystems Wald mit all ihren ökologischen charakteristischen Eigenarten und Prozessen in all ihren Raum- und Zeiteinheiten und unter Berücksichtigung auch historisch bedingter Forstökosysteme (Mittel-, Niederwälder u.a.) zu gewährleisten“ (STURM 1993). Neben dem Schutz der Prozesse als Primärziel werden klassische Naturschutzziele (zum Beispiel 
Vielfalt oder Stabilität) zu sekundären Zielen. Prozeßschutz wird als Waldschutz verstanden, der nicht vorrangig Zustände, sondern Entwicklungsbedingungen erhält. Im besonderen sollen auch Pionier- und Zwischenwaldphasen ermöglicht werden. Sie gelten als wichtige Elemente eines multivariablen, zufallsbeeinflußten Sukzessionsmosaiks. Ereignisse wie Windwurf, Feuer, Überschwemmung oder Insektengradation werden als Element des Prozeßschutzes verstanden (siehe STURM 1994). Über langfristige Artenzusammensetzungen kann bei einem Schutz der dynamischen Abläufe nur spekuliert werden. Waldbauliche Zieltypen, wie sie zum Beispiel als Waldentwicklungstypen im LÖWE-Konzept beschrieben werden, können im Sinne des Prozeßschutzes nicht eingesetzt werden.

Das hier vorgestellte Entwicklungsszenario ist nicht im Sinne einer vollständigen Umsetzung des von Sturm und Fähser beschriebenen Prozeßschutzmodells zu verstehen. Die Modellbeschreibungen sind zwar umfangreich, aber für den Einzelfall noch nicht ausreichend konkret. Gerade die Anweisung, das waldbauliche Handeln an von der Nutzung ausgenommenen Referenzflächen auszurichten, läßt viele Fragen offen. Es wären Einzelfallentscheidungen zu treffen, die programmiertechnisch nicht faßbar sind. Auch grundsätzliche Zweifel an der Umsetzbarkeit und der Sinnhaftigkeit des Referenzflächenansatzes führten zu der Entscheidung, dieses Element nicht in das Modell einfließen zu lassen. Meyer und Spellmann (siehe MeYer und Spellmann 1997) gehen zum Beispiel auf die hohen Kosten betriebsinterner Referenzflächen ein, die eine unzureichende Aussagekraft besitzen. Sie kritisieren nicht existente Aufnahme- und Auswertungsregelungen und ungenügende Beobachtungsdauer. Weiterhin vertreten sie die Meinung, daß eine Verallgemeinerung der Ergebnisse betriebsinterner Referenzflächen nicht zu leisten ist. Abgesehen von dieser Einschränkung übernimmt die Variante PROZESS wesentliche Aspekte des Prozeßschutzmodells. Sie minimiert forstliche Eingriffe und ermöglicht dadurch maximalen Raum für natürliche Waldentwicklungsprozesse bei gleichzeitiger wirtschaftlicher Nutzung.

Die natürlichen Prozesse im Wald werden genutzt und unterstützt, indem zum Beispiel konkurrenzstarke Bäume zusätzlich gefördert werden. Dabei unterscheidet das umgesetzte Modell jedoch nicht zwischen Fremdländern und einheimischen Arten. Die Förderung von Bäumen durch die Entnahme ihrer Bedränger fällt im Vergleich zu den anderen Szenarien eher schwach aus. Es wird mit wenigen Eingriffen (alle zehn Jahre) gearbeitet, um dem Bestand die Möglichkeit zu geben, sich zwischen diesen Behandlungen natürlich zu differenzieren.

Die natürliche Entwicklungsdynamik wird auch dadurch nicht aktiv beeinflußt, daß bestimmte Baumartenmischungsverhältnisse angestrebt, oder Pflanzungen vorgenommen werden. In der Simulation werden selbst naturferne Bestände nicht aktiv umgesteuert.

Auf Kahlschläge wird gänzlich verzichtet, um in der Endnutzungsphase nur kleinflächige Störungen zu verursachen. Zielstarke Bäume werden individuell genutzt. Dieses Vorgehen reduziert die Verjüngungsmöglichkeiten für lichtbedürftige Baumarten. Lichtbaumarten wie die Eiche werden durch die fehlende Förderung immer weiter zurückgedrängt werden. Es ist zu erwarten, daß im Untersuchungsgebiet die Kiefer zunehmend durch Buche ersetzt 
wird, aber auch die in der Verjüngung konkurrenzstarke Fichte einen erheblichen Anteil der Fläche einnehmen wird.

Der Forderung, 10\% aller Bäume von der Nutzung auszunehmen (STURM 1993) nähert sich die Simulation lediglich an, indem ein Habitatbaum pro Hektar ausgewählt und somit der Nutzung entzogen wird.

\subsection{Untersuchungsgegenstände}

Die Szenariensimulation ist auf die Auswertung der Entwicklung einer Landschaft oder eines Betriebs ausgerichtet. In dieser Arbeit wird beispielhaft das hier vorgestellte Untersuchungsgebiet (siehe Kapitel 3.2.1) fortgeschrieben. Um die Plausibilität dieser Ergebnisse zu stützen, werden die im letzten Kapitel vorgestellten Szenarien in für das Untersuchungsgebiet typischen Beispielbeständen (siehe Kapitel 3.2.2) eingesetzt. Anhand dieser Beispielbestände können Auswirkungen der szenarienabhängigen Eingriffe veranschaulicht werden.

\subsubsection{Untersuchungsgebiet}

Der gewählte Untersuchungsraum in der Lüneburger Heide, der im folgenden kurz als Untersuchungsgebiet „Lüneburger Heide“ oder „Heide“ bezeichnet wird, liegt zwischen den Städten Hamburg und Hannover. Er befindet sich in den Wuchsbezirken „Hohe Heide“ und "Ost-Heide“. Die geologischen Charakteristika dieser Wuchsbezirke werden in Abschnitt „Geologie/ Relief“ beschrieben, die klimatischen Voraussetzungen in Abschnitt „Klima“.

Da das Untersuchungsgebiet Objekt von zwei vorangegangenen Forschungsprojekten ${ }^{11}$ war, liegt hier eine breite Datengrundlage und detailliertes Wissen über die waldbauliche Ausgangssituation vor. Die Ergebnisse des deutschen Teilprojekts „Nachhaltige multifunktionale Waldentwicklung“ aus dem EU-Life Projekt „Demonstration of Methods to Monitor Sustainable Forestry“ wurden 2002 (NFV UND NFA 2002) und das DBU-Projekt "Nachhaltige und multifunktionale Forstwirtschaft" 2004 (SPelLmANN ET AL. 2004) veröffentlicht. Die Datengrundlage zeigt, daß die Möglichkeiten durch waldbauliche Maßnahmen strukturverändernd auf die Untersuchungsgebiete Einfluß zu nehmen, gerade in diesem Gebiet sehr groß sind (siehe auch Kap. 3.2.1: Waldbauliche Situation). Das Untersuchungsgebiet setzt sich aus Teilen des Niedersächsischen Forstamts Sellhorn (ca. 4900ha), der Forstbetriebsgemeinschaft (FBG) Egestorf-Hanstedt (ca. 2200ha) und des Klosterforstamts Soltau (ca. 4600ha) zusammen (siehe Abbildung 3.2.1.a).

Diese drei Besitzarten unterscheiden sich in ihrer Organisationsstruktur (Niedersächsisches Forstamt, Landesbetrieb, wirtschaftlicher Verein) und verfolgen verschiedene Zielsetzungen. Die Flächen des staatlichen Forstamts werden nach dem Programm der Landesregierung Niedersachsen „LÖWE“ (Langfristige ökologische Waldentwicklung (NiedeRsÄCHSISCHE LANDESFORSTVERWALTUNG 1991)) bewirtschaftet. Dieses naturnahe Waldbaukonzept wird in den Kapiteln 3.1.1. und 3.5.1 ausführlich beschrieben, da es im Rahmen des hier entwickelten Programms umgesetzt wurde. Sowohl das Klosterforstamt als auch das Gebiet der FBG wurden in der Vergangenheit in Anlehnung

11 Die Flächen entsprechen sich zu 95\%. 


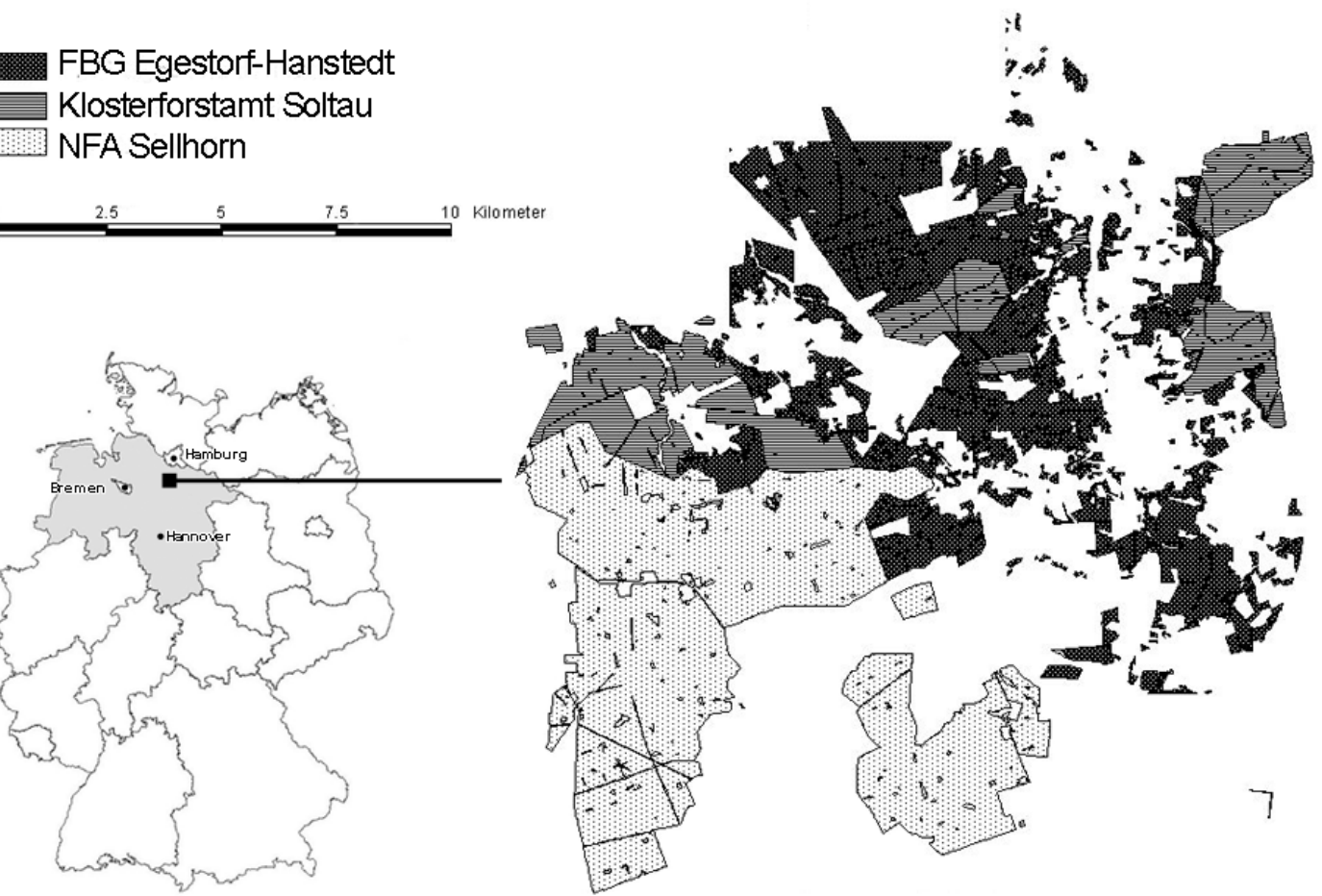

\section{Abbildung 3.2.1.a: Das Untersuchungsgebiet Lüneburger Heide mit getrennt} ausgewiesenen Besitzarten.

an das LÖWE-Programm bewirtschaftet (SPELLMANn ET AL. 2004). Bei der Bewirtschaftung der Flächen des Klosterforstamts wird jedoch besonders auf das Erzielen eines hohen Reinertrags bei Erhaltung der Wertnachhaltigkeit geachtet. Auch für viele der bäuerlichen Eigentümer der Forstbetriebsgemeinschaft stehen monetäre Erträge im Vordergrund, da der Wald für sie eine wichtige Einkommensquelle darstellt. Mit der Vielzahl der Eigentümer, die in dem wirtschaftlichen Verein zusammengeschlossen sind, geht auch einher, daß verschiedene Zielvorstellungen bei den Mitgliedern existieren. Die Betreuung der FBG Egestorf-Hanstedt ist mit einem hohen Koordinationsaufwand bei der Bewirtschaftung verbunden, da sie sich aus über 200 Eigentümern zusammensetzt, deren Waldbesitz eine durchschnittliche Größe von 35,8ha hat (siehe SpelLmAnN ET AL. 2004).

An das entwickelte Programm stellte sich durch die heterogene Datenlage in den verschiedenen Besitzarten die Anforderung, daß es auf einer gängigen Datenbasis aufsetzen muß, die sich als "kleinster gemeinsamer Nenner" der verschiedenen Datengrundlagen herauskristallisieren läßt.

\section{Geologie/ Relief}

Das Untersuchungsgebiet liegt in einer Altmoränenlandschaft, die sich im Warthe-Stadium des Saaleglazials gebildet hat. Die Region wird von ebenen Grundmoränenzügen, kuppigen Endmoränenzügen, der Eisfront vorgelagerten Schmelzwassersanden und Beckentonen geprägt (ARBEITSKREIS STANDORTSKARTIERUNG 1985). Nacheiszeitliche Ablagerungen (Flußsande, Torfe, Flugdecksande und Dünen) finden sich vorwiegend in Talniederungen. Diese Täler findet man häufig im Nordosten, sie sind charakteristisch für 
die „Hohe Heide“. Das Relief nimmt allgemein vom flachwelligen Südwesten nach Nordosten zu. Dabei bewegt man sich meist auf einer Höhe von 80-100 Metern (siehe Bundesanstalt für Geowissenschaften und Rohstoffe 1977 und Hinze et al. 2006)

\section{Böden}

Seit der Entstehung der Böden vor 140.000 Jahren wirkten Erosion und Verwitterung, aber auch anthropogene Einflüsse auf sie ein. Im besonderen sind hier „Plaggenhiebe“ zu nennen, die im Mittelalter oft angewendet wurden und bei denen der Oberboden regelmäßig abgetragen wird. Das führt zu einer Degradation des Bodens und zur Ausbreitung der Heide, bei deren Zersetzung der pH-Wert des Bodens stark absinkt. Dadurch werden Eisenhydroxide, Aluminiumhydroxide und Huminstoffe mit dem Sickerwasser ausgewaschen - die sogenannte Podsolierung. Dabei entstanden aus den höhenpleistozänen Sanden meist reine Podsole oder Podsol-Braunerden. Diese schwach bis mäßig versorgten Standorte sind stauwasserfrei, und das Grundwasser befindet sich auf einem niedrigen Level. Unter Einfluß von Grundwasser entwickelten sich in dem Gebiet Gleye (Gleye oder Podsol-Gleye). Dort wo Geschiebelehme einen verdichteten

Stauhorizont bilden, entstanden Pseudogleye (Pseudogley-Braunerden, PseudogleyPodsole). Auf silikatreicheren Sanden bildeten sich teilweise auch Braunerden, in den im Holozän geprägten Teilen findet man auch Eschböden und Dünen (CORDES ET AL. 1997 und SpelLmanN ET AL. 2004).

\section{Klima}

Allgemein kann das Klima als Übergangsklima zwischen Küsten- und Binnenland bezeichnet werden. Dabei ist der Wuchsbezirk "Hohe Heide“ deutlich ozeanischer geprägt als die „Ost-Heide“.

Tabelle 3.2.1.a: Klimadaten des Wuchsbezirks „Hohe Heide“ im Vergleich zu benachbarten Wuchsbezirken (zum Teil aus VEREIN FÜR FORSTLICHE STANDORTSKUNDE UND FORSTPFLANZENZÜCHTUNG 2005).

\begin{tabular}{lll}
\hline Klimadaten & Hohe Heide & Ost-Heide \\
\hline Jahresniederschläge & $761 \mathrm{~mm}$ & $657 \mathrm{~mm}$ \\
\hline $\begin{array}{l}\text { Niederschläge in der Vegetationszeit } \\
(1.5 .-30.9 .)\end{array}$ & $346 \mathrm{~mm}$ & $307 \mathrm{~mm}$ \\
\hline $\begin{array}{l}\text { Klimatische Wasserbilanz in der forstlichen } \\
\text { Vegetationszeit (1.5. - 30.9.) }\end{array}$ & $-95 \mathrm{~mm}$ & $-150 \mathrm{~mm}$ \\
\hline Mittlere Jahrestemperatur & & \\
\hline Mittlere Temperatur in der Vegetationszeit & $8,4^{\circ} \mathrm{C}$ & $8,5^{\circ} \mathrm{C}$ \\
\hline Zahl der Tage mit $>=10^{\circ} \mathrm{C}$ & $14,7^{\circ}$ & $15^{\circ} \mathrm{C}$ \\
\hline
\end{tabular}

Der Höhenzug der „Hohen Heide“ (Endmoräne mit mehreren Erhebungen über 150m) bedingt in diesem Teil niedrigere Temperaturen und höheren Niederschlag, als sie im Umland auftreten. Dieses Regionalklima wird auch als „kleines Berglandklima“ bezeichnet (Отто 1989). In der „Ost-Heide“ liegen die Jahresniederschläge mit 657mm gut 100mm 
niedriger. Weiterhin unterscheidet sich die mittlere Temperatur während der Vegetationszeit $\left(14,7^{\circ} \mathrm{zu} 15^{\circ}\right)$ in den beiden Gebieten (siehe Tabelle 3.2.1.a).

\section{Waldgeschichte}

Im Untersuchungsgebiet spiegelt sich die Natur-, Kultur- und Waldgeschichte der Heide und des norddeutschen Tieflands wider. Bereits in der Bronzezeit wurden viele Waldflächen intensiv beweidet, und in dem ehemals geschlossenen Waldgebiet (aus Buchen und Eichen) entstanden durch halbwilde Herden von Rindern, Schweinen, Ziegen, Schafen und Pferden offene Flächen (KREMSER 1972). Beweidungsresistente Pflanzenarten wie die Besenheide (Calluna vulgaris) siedelten sich an.

Nachdem der Waldanteil während der Völkerwanderungszeit kurzfristig wieder gestiegen war, kam es seit dem Mittelalter in Folge der Dauerbesiedlung des Gebiets zu einer Intensivierung der Landwirtschaft. Die Wälder wurden einerseits als Brennholzlieferant, zunehmend aber auch als Weideflächen genutzt, was dazu führte, daß im Wald keine Verjüngung aufkommen konnte, da sie dem Vieh als Futter diente. Außerdem wurde beim „Laubbrechen“ die Streuschicht des Waldes mitsamt der Humusdecke entnommen, um sie als Einstreu in Ställen zu verwenden. Somit verarmten die Wälder, sie wurden immer lichter und die Heidefläche nahm stark zu. Selbst die Heideflächen wurden stark landwirtschaftlich genutzt, was zu einer weiteren Verarmung des Bodens führte. Beim sogenannten „Abplaggen“ wurden die Heidesträucher mit ihrem Wurzelwerk herausgestochen und so dem Boden ständig Nährstoffe entzogen. Die „Plaggen“ wurden als Stallstreu, Dünger und zur Befestigung von Reetdächern genutzt (siehe CORDES ET AL. 1997).

Die wenigen verbleibenden Wälder (aus Buche und Traubeneiche) waren stark aufgelichtet, und deshalb fehlte es vor allem an Bauholz. Ein Problem stellten auch die freigelegten Sandböden dar: Um die großen Wehsandflächen festzulegen und um dem Holzmangel zu begegnen, wurden seit dem 18. Jahrhundert Aufforstungen betrieben. Von einer „Aufforstungswelle“ kann man aber erst ab der Mitte des 19. Jahrhunderts sprechen (Cordes et Al. 1997).

Auf den armen Heideböden war lediglich die Baumart Kiefer in der Lage, mit den örtlichen Bedingungen wie Dürre, Frost, Wind und Wehsanden sowie der Vegetationskonkurrenz durch Calluna Vulgaris fertig zu werden. Auf besseren Böden konnten auch Fichten gepflanzt werden; die Wiederansiedlung von Eichen gelang nur selten auf ehemaligen Ackerböden. Anpflanzungen von Buchen (die vormals das Gebiet dominierten) waren auf den offenen Flächen nicht möglich. Ein höherer Buchen- und Eichenanteil sollte daher erst in der zweiten Waldgeneration angestrebt werden (KREMSER 1990). Dieser Umbau konnte bis heute noch nicht vollzogen werden, da große Teile der Kiefernaufforstungen nach dem Zweiten Weltkrieg in den sogenannten „Reparationshieben“ kahlgeschlagen wurden. Auch waren die großflächig angelegten, gleichaltrigen Kiefernreinbestände eine ideale Umgebung für Insektenmassenvermehrungen, so daß ein Teil des Waldes sich nicht regenerieren konnte. Weiterhin fielen einige Flächen Bränden und Windwürfen zum Opfer. Auf den Freiflächen wurde wieder vorzugsweise Kiefer gepflanzt, da sie für andere Baumarten nicht geeignet waren. 
Da die Fichte im Gegensatz zur Buche in der Lage ist, sich relativ weit und rasch natürlich zu verjüngen, nahm im Laufe der Zeit der Anteil an Fichte deutlich zu. Mit der Abkehr vom Kahlschlag hin zu Einzelbaumnutzung und einer angemessenen Bejagung erhöhte sich seit dem Ende des 20. Jahrhunderts der Laubwaldanteil stetig. Auch der Weichlaubholzanteil stieg wieder an, da Nebenbaumarten nicht mehr entnommen werden, sondern sogar erwünscht sind.

\section{Standorte/ Potentiell natürliche Vegetation}

Der Großteil der Bestandesflächen im Untersuchungsgebiet (48\%) wird von sehr schwach oder schwach nährstoffversorgten Standorten dominiert. Mäßig versorgte Standorte nehmen noch $40 \%$ der Bestandesflächen ein. Eine ziemlich gute Nährstoffversorgung kommt lediglich auf $12 \%$ der Fläche vor. Bestände, die vorwiegend auf sehr gut versorgten Böden stocken, findet man im Untersuchungsgebiet gar nicht.

Über $70 \%$ der Fläche werden von mäßig frischen, mäßig sommertrockenen oder trockenen Beständen eingenommen. Als wechseltrocken, nachhaltig frisch bzw. frisch bis vorratsfrisch sind nur ca. 18\% der Fläche zu bezeichnen. 11\% der Bestände stocken auf überwiegend grundnaß bis staufeuchten Böden, und auf bis zu einem Prozent der Fläche findet man Moore vor.

Die Beschreibung der Nährstoff- und Wasserversorgung sowie die Zuordnung einer potentiell natürlichen Vegetation wurden anhand der Bestandesflächen erarbeitet. Der Bestand wurde der jeweils flächenmäßig dominierenden Kennziffer zugerechnet. Als natürliche Waldgesellschaft dominieren großflächig Drahtschmielen-Buchenwälder. In trockeneren Teilbereichen sind Drahtschmielen-Buchenmischgesellschaften mit Traubeneichen sehr wahrscheinlich. Auf den trockenen und armen Standorten würde die natürliche Bestockung aus Birken-Eichenwäldern bestehen (siehe auch KAISER UND ZACHARIAS 2003).

\section{Waldbauliche Situation}

Das Waldbild der Lüneburger Heide wandelt sich seit den letzten 200 Jahren von gleichaltrigen Reinbeständen hin zu strukturreicheren, naturnäheren Mischbeständen (siehe Abschnitt „Waldgeschichte“). Die Geschichte des Waldes mit seiner Entstehung aus großflächigen Kiefernerstaufforstungen spiegelt sich auch heute noch in seiner Artenzusammensetzung, der Bestandes- und Landschaftsstruktur und seinem Entwicklungspotential wider. Die heutige Verteilung der Bestandestypenanteile gemäß der Definition des Waldwachstumssimulators BWINPro (siehe Glossar) zeigt, daß die kieferndominierten Bestandestypen, die in der Heide als sukzessionales Stadium der Waldentwicklung begriffen werden können, auf $61 \%$ der Fläche zu finden sind. Laubwalddominierte Bestände, die der potentiell natürlichen Vegetation entsprechen, haben hingegen nur einen Anteil von ca. 11\% (siehe Abbildung 3.2.1.b). Bestände aus ertragsstärkeren Baumarten wie Fichte oder auch die eingeführte Douglasie nehmen gemeinsam einen Anteil von 23\% ein. 

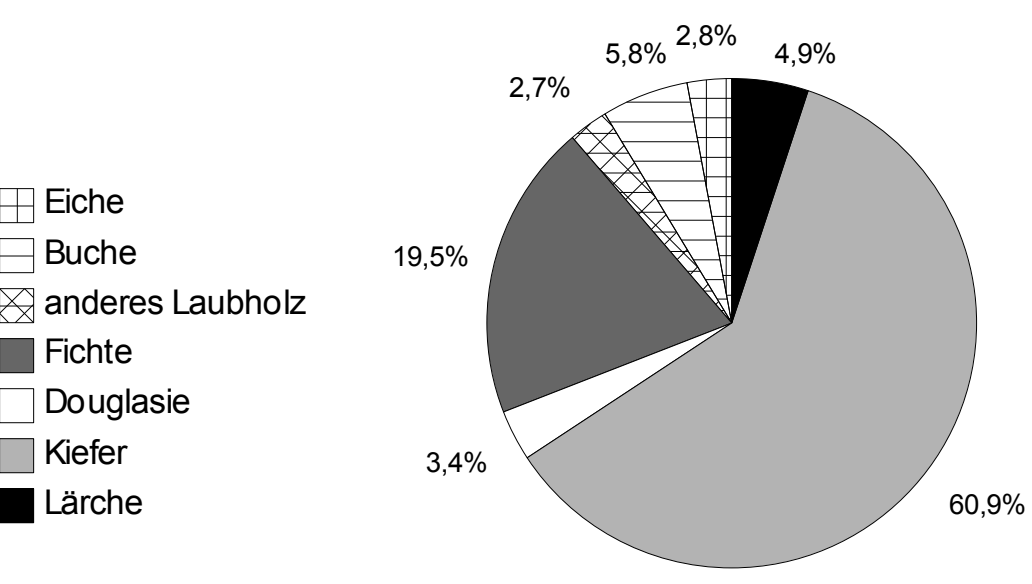

Abbildung 3.2.1.b: Flächenanteile der Bestandestypengruppen im Untersuchungsgebiet.

Der mit einem Waldumbau verbundene gesteuerte Baumartenwechsel vollzieht sich in den Beständen immer dann, wenn durch Erntemaßnahmen eine natürliche Verjüngung der Bestände eingeleitet wird, bzw. eine künstliche Verjüngung erforderlich ist. Gegebenenfalls werden Baumarten, die bis dato noch nicht im Bestand vorkommen, auch durch Voranbau eingebracht, der unter den Schirm des Altbestandes gepflanzt wird.

Aus Voruntersuchungen ist bekannt, daß $52 \%$ des Vorrats zwischen 20 und 60 Jahre alt sind, wobei die Altersklasse III mit 32,3\% den größten Anteil einnimmt (NFV UND NFA 2002, siehe auch Abbildung 3.2.1.c). Da Endnutzungsmaßnahmen in der Kiefer verstärkt in die Altersklasse V (81-100 Jahre) eingreifen, kann man davon ausgehen, daß neue Baumarten in den nächsten vierzig bis sechzig Jahren effektiv eingebracht werden können.

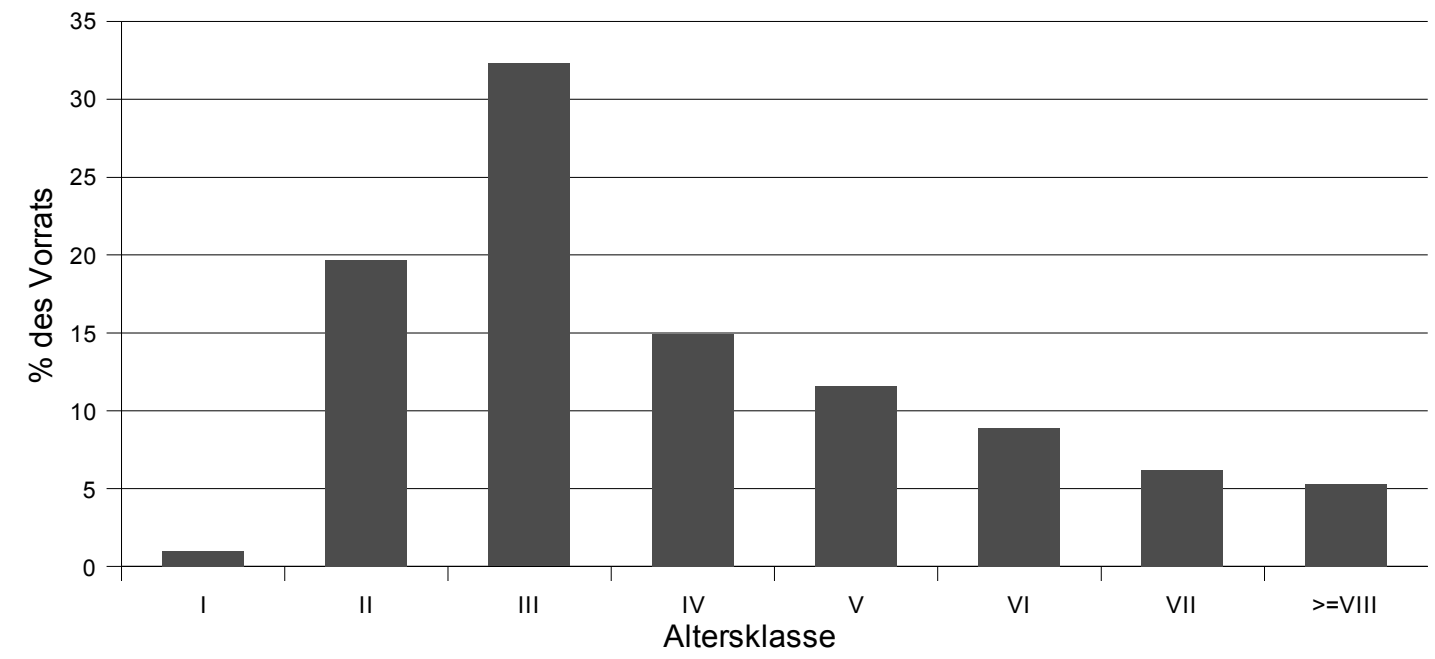

Abbildung 3.2.1.c: Vorratsstruktur im Untersuchungsgebiet des EU-Life Projekts „Demonstration of Methods to Monitor Sustainable Forestry - Nachhaltige multifunktionale Waldentwicklung ", nach Altersklassen in Prozent des Gesamtvorrats (nach NFV UND NFA 2002). 
Aus Alterklassenverteilung und Baumartenzusammensetzung wird deutlich, daß das Waldumbaupotential derzeit hoch ist. In der heutigen Situation eignet sich das Untersuchungsgebiet demnach besonders gut für eine Analyse der weiteren Waldentwicklung bei Verfolgung verschiedener Waldbaukonzepte.

\section{Datengrundlage des Untersuchungsgebiets}

Um die Entwicklung eines Betriebs oder einer Landschaft simulieren zu können, werden Inventurdaten benötigt, die über die Zusammensetzung der Bestände und die Verjüngungssituation Aufschluß geben. Für das Untersuchungsgebiet liegen flächendeckend Inventurdaten aus der jeweiligen Forsteinrichtung vor.

Die Forsteinrichtungsdaten aus dem Staatswald stammen aus dem Jahr 1998. Sie wurden nach der „Anweisung zur Betriebsregelung (Forsteinrichtung) in den Niedersächsischen Landesforsten (B.A.87)“ aufgenommen (NIEDERSÄCHSISCHE LANDESFORSTVERWALTUNG 1987). In Anlehnung an diese Richtlinie wurden auch im Klosterforstamt Soltau und der FBG Egestorf-Hanstedt Daten mit den Stichjahren 2000 und 2002 erhoben. Zwar unterscheiden sich die Aufnahmen in den drei Besitzarten teilweise, jedoch werden die Ansprüche, die das Programm an die Daten stellt, von allen erfüllt. Es basiert auf Grundlagendaten, die in der Regel in mittleren und großen Forstbetrieben erhoben werden. Die Betriebe nutzen diese Informationen einerseits, um steuerlichen Anforderungen (siehe § 34 EStG ESTG 2005) gerecht zu werden. Andererseits orientieren sich ihre innerbetrieblichen Planungen an diesen Werten. Die flächendeckende Waldinventur ist in vielen Fällen sogar zum wichtigsten Instrument der Privatwaldbetreuung geworden (HILLMANN 2002 und HiLLMANN 2006), und die Forsteinrichtung wird zunehmend als strategisches Managementinstrument gesehen (HANEWINKEL 2001).

\subsubsection{Beispielbestände}

Als Beispielbestände kommen in erster Linie häufig vorkommende Bestandestypen in Frage, deren führende Bestandesschicht sich in einer für das Gebiet typischen Altersklasse befindet. Unter den fünf häufigsten Bestandestypen gemäß der simulationsintern verwendeten Definition befinden sich reine Kiefernbestände mit einer Anteilfläche am Untersuchungsgebiet von 38\%, Kiefern-/ Fichtenbestände mit $13 \%$, Fichten-/ Kiefernbestände mit 10\%, Kiefernbestände mit sonstigem Laub- und Nadelholz mit $8 \%$ und reine Fichtenbestände mit $8 \%$. Weitere Bestandestypen haben lediglich einen jeweiligen Anteil am Untersuchungsgebiet, der unter $3 \%$ liegt. Jeweils ein Kiefern- und ein Fichtenreinbestand sowie ein Kiefern-/ Fichtenmischbestand werden vorgestellt, um Situationen für Rein- und Mischbestände aus den beiden Baumarten abzubilden. Auf den Typ „Kiefernbestände mit sonstigem Laub- und Nadelholz“ wird nicht näher eingegangen, da er unterschiedliche Mischungstypen zusammenfaßt und nicht durch eine bestimmte Baumartenzusammensetzung repräsentiert werden kann.

Stellvertretend für die Kiefernreinbestände des Untersuchungsgebiets wird ein Bestand der Altersklasse III (von 40-60 Jahren) gewählt, da diese Altersklasse die dominierende Altersklasse reiner Kiefernbestände ist. Sie liegt mit einen Flächenanteil von $50 \%$ an der 
von Kiefernreinbeständen bestockten Fläche deutlich vor der nächststarken Altersklasse II (von 21-40), die lediglich 15\% ausmacht.

Die Fichtenreinbestände des Untersuchungsgebiets werden durch einen Beispielbestand repräsentiert, der der zweiten Altersklasse angehört. Diese Altersklasse nimmt mit 36\% den höchsten Anteil der mit Fichtenreinbeständen bestockten Fläche ein.

Der Beispielbestand Kiefer/ Fichte steht stellvertretend für die Bestandestypen Kiefer/ Fichte und Fichte/ Kiefer. Auch in Mischbeständen aus den beiden Baumarten gehören die meisten Kiefern der Altersklasse III, die meisten Fichten der Altersklasse II an. Der repräsentierende Mischbestand setzt sich entsprechend aus Kiefern der Altersklasse III und Fichten der Altersklasse II zusammen.

Tabelle 3.2.2.a: Übersicht über die Eingangswerte für den Aufbau der vier Beispielbestände.

\begin{tabular}{|c|c|c|c|c|c|c|c|c|}
\hline $\begin{array}{l}\text { Beispiel- } \\
\text { bestand }\end{array}$ & Baumart & $\begin{array}{l}\text { Alter } \\
\text { (Jahre) }\end{array}$ & $\begin{array}{l}D g \\
(\mathrm{~cm})\end{array}$ & $\begin{array}{l}\mathrm{Hg} \\
(\mathrm{m})\end{array}$ & $\begin{array}{l}D \max \\
(\mathrm{cm})\end{array}$ & $\begin{array}{l}\text { Grund- } \\
\text { fläche } \\
\left(m^{2} / h a\right)\end{array}$ & $\begin{array}{l}\text { absolute } \\
\text { Bonität } \\
\text { (m) }\end{array}$ & $\begin{array}{l}\text { Flächenanteil } \\
\text { Pflanzungen } \\
\text { (\% Bestandesfläche) }\end{array}$ \\
\hline 1 & $\begin{array}{l}\text { Pinus } \\
\text { sylvestris }\end{array}$ & 50 & 18,6 & 17,4 & 24,2 & 31,3 & 28,0 & \\
\hline 2 & Picea abies & 30 & 9,7 & 8,6 & 12,6 & 27,2 & 31,2 & \\
\hline \multirow[t]{2}{*}{3} & $\begin{array}{l}\text { Pinus } \\
\text { sylvestris }\end{array}$ & 50 & 18,6 & 17,4 & 24,2 & 15,6 & 28,0 & \\
\hline & Picea abies & 30 & 9,7 & 8,6 & 12,6 & 13,6 & 31,2 & \\
\hline \multirow[t]{2}{*}{4} & $\begin{array}{l}\text { Pinus } \\
\text { sylvestris }\end{array}$ & 110 & 35,4 & 27,1 & 49,6 & 32,8 & 28,0 & keine \\
\hline & $\begin{array}{l}\text { Fagus } \\
\text { sylvatica }\end{array}$ & 15 & & & & & 29,4 & 60 \\
\hline
\end{tabular}

Die mittlere Ertragsklasse der Kiefer liegt im Untersuchungsgebiet zwischen I und II, die Fichte ist meist der Ertragsklasse II zuzuordnen. Die Eingangsdaten für die Beispielbestände orientieren sich an den Bestandesmittelwerten vollbestockter Bestände, der Ertragstafel von Schober (siehe ScHOBER 1975) für mäßig durchforstete Kiefer der Ertragsklasse I,5 und mäßig durchforstete Fichte der Ertragsklasse II. Die übergebenen Werte sind in Tabelle 3.2.2.a zu finden.

Die Summe der Flächen, die Kiefernbeständen der dritten Altersklasse, Fichtenbeständen der zweiten Altersklasse und Kiefern-/ Fichtenbeständen oder Fichten-/ Kiefernbeständen der zweiten bzw. dritten Altersklasse zugerechnet werden, beträgt $57 \%$ des Untersuchungsgebiets. Somit repräsentieren sie einen Großteil der untersuchten Fläche. Aufgrund der vertretenen Altersklassen können jedoch nur Auswirkungen von Durchforstungseingriffen gezeigt werden.

Um die unterschiedlichen Endnutzungsstrategien anhand typischer Bestände vorzustellen, wird ein mit Buchen unterbauter hundertzehnjähriger Kiefernbestand herangezogen (siehe Beispielbestand 4 in Tabelle 3.2.2.a). Da sich nur ein kleiner Teil des Untersuchungsgebiets im Endnutzungsalter befindet, repräsentiert dieser Bestand nur 
einen Bruchteil (ca. 2\%) des Untersuchungsgebiets. Für einen Endnutzungsbestand ist er aber dennoch charakteristisch, da er den überwiegenden Teil der Bestände dieser Altersklasse abbildet.

\section{3 Überblick über Simulation und Auswertung}

\subsubsection{Auswahl und Weiterentwicklung eines geeigneten Simulationsinstruments}

Wie in Kapitel 2.3.2 gezeigt, sind Einzelbaumwachstumssimulatoren ein geeignetes Instrument, um waldbauliche Managementstrategien zu vergleichen. Sie können die Auswirkungen forstlicher Eingriffe über einen mittelfristigen Zeitraum anhand multifunktionaler Kriterien darstellen. In dieser Arbeit wird auf den Waldwachstumssimulator BWINPro (DöBBELER ET AL. 2003 und NAgEL 2002) zurückgegriffen. BWIN wird schon seit einigen Jahren von der Niedersächsischen Landesforstverwaltung verwendet (siehe Böckmann 2004 a). Die dieser Arbeit zugrunde liegenden Wachstumsroutinen stehen unter dem Namen TreeGrOSS (Tree Growth Open Source Software (NAGEL 2002)) als Open Source Software unter der General Public Licence GNU zur Verfügung.

Für das Programm BWINPro spricht einerseits, daß es anhand von Versuchsflächen der Nordwestdeutschen Forstlichen Versuchsanstalt parametrisiert worden ist. Die implementierten Wuchsroutinen können daher die Bedingungen im Untersuchungsgebiet (Lüneburger Heide) abbilden. Andererseits erleichtert der in der Programmiersprache JAVA umgesetzte objektorientierte Aufbau des Programms eine problemorientierte Zusammenstellung der Einzelkomponenten.

Die in Kapitel 1.2 vorgestellten Ziele können verwirklicht werden, indem den Wachstumsroutinen ergänzende Routinen hinzugefügt werden. Dabei ist der Programmierschritt zur automatisierten Verarbeitung mehrerer Bestände ein Schlüsselfaktor, um den auf Einzelbestände bezogenen Simulator als Managementtool auf strategischer Ebene verwenden zu können (siehe auch voN TEUFFEL ET AL. 2006).

Die in dieser Arbeit umgesetzte Automatisierung, die eine Verarbeitung ganzer Betriebe oder Landschaften ermöglicht, umfaßt:

\section{Die Verarbeitung und Aufbereitung von Eingangsdaten}

Zur Verarbeitung und Aufbereitung von Eingangsdaten wurde eine Schnittstelle zu einer Datenbank und Datenergänzungsroutinen erstellt.

\section{Das Festlegen der szenarioabhängigen Parameter und das Treffen von szenarioabhängigen Eingriffsentscheidungen}

Um verschiedene Szenarien darzustellen, bedurfte es der Umsetzung und Implementierung forstlicher Eingriffe, die zu der jeweiligen Managementstrategie gehören. 


\section{Die Fortschreibung des Wachstums der Bestände}

Die Fortschreibung des Wachstums wurde um eine Einwuchsroutine erweitert, die Einwuchs aus Pflanzungen und Einwuchs aus zum Simulationsstart bekannter Naturverjüngung ermöglicht.

\section{Die Ausgabe der Ergebnisse}

Es wurden multifunktionale Bewertungskriterien erarbeitet und entsprechende Auswertungsalgorithmen programmiert.

\subsubsection{Verarbeitungsschritte und Datenhaltung}

Entlang der Verarbeitungsschritte, die den Vergleich der Managementstrategien ermöglichen, wird in diesem Kapitel die Datenhaltung beschrieben.

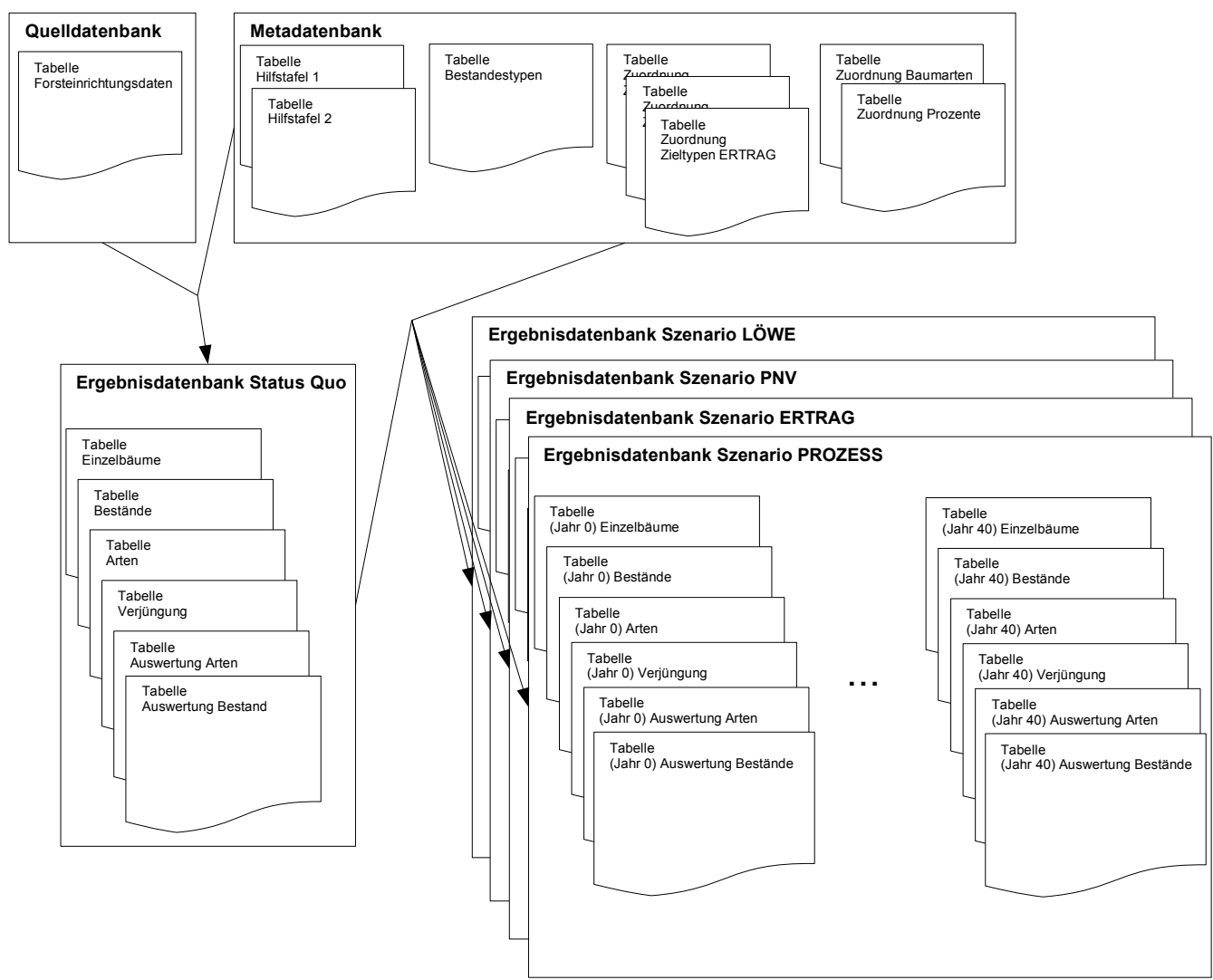

Abbildung 3.3.2.a: Datenbankaufbau der Simulation. Der Aufbau der Ergebnisdatenbank kann optional gesteuert werden. Der Benutzer kann individuell wählen, welche Zwischenergebnis- und Endergebnistabellen er festhalten möchte.

Sowohl Ausgangsdaten als auch Ergebnisse werden in Datenbanken gespeichert. Die Wahl fiel auf das Datenbankprogramm PostgreSQL, da das Open Source GIS (Geographisches Informationssystem) GRASS eine komfortable Schnittstelle zu diesem Programm bietet. Die Ergebnisse können somit mit einem für jedermann zugänglichen GIS 
auf Landschaftsebene ausgewertet werden. Abbildung 3.3.2.a zeigt die Untergliederung der Datenbanken in Datenbanktabellen.

Der Weg zum Vergleich der forstlichen Managementstrategien gliedert sich in drei Schritte (siehe Abbildung 3.3.2.b):

(1) Datenvorbereitung und Ausgabe der Ausgangssituation (siehe Kapitel 3.4)

(2) Szenarienabhängige Fortschreibung und Ausgabe der Simulationsergebnisse (siehe Kapitel 3.5)

(3) Auswertung (siehe Kapitel 3.6)

Zu (1): Zunächst werden Bestandesdaten aus der Forsteinrichtung in den Waldwachstumssimulator eingelesen und fehlende Daten werden ergänzt (siehe Kapitel 3.4.1). Die Ausgangsdaten der Forsteinrichtung müssen in einer Quelldatenbank (siehe Abbildung 3.3.2.a) in Form einer Tabelle vorliegen. Bei der Datenergänzung wird auf Hilfstafeln (NiedeRsÄCHSISCHES ForStPLANUNGSAMT 1987) zurückgegriffen, die in einer sogenannten Metadatenbank abgelegt sind (siehe Abbildung 3.3.2.a). Diese Hilfstafeln stellen eine Beziehung zwischen Baumart, Alter, Leistungsklasse und Bestandeskennzahlen wie Dg, Hg oder Grundfläche (Definition siehe Glossar) her. Ihnen liegen die Ertragstafeln von Schober zu Grunde (ScHовеR 1975). Der Ausgangszustand wird nach der Datenaufbereitung in den Tabellen „Einzelbäume“, „Bestände“, „Arten“ und „Verjüngung“ der Ergebnisdatenbank „Status Quo“ gespeichert.

Anschließend wird der Ausgangszustand auf Arten- und Bestandesebene ausgewertet (siehe Kapitel 3.4.2). Bei der Auswertung der Bestandesinformationen wird auf die Tabellen „Bestandestypen“, „Zieltypen“, „Zuordnung Baumarten“ und „Zuordnung Prozente" der Metadatenbank zugegriffen. Speicherort für die Auswertung der Ausgangssituation ist wieder die Ergebnisdatenbank für den Status Quo (siehe Abbildung 3.3.2.a). Es werden zwei Auswertungstabellen gespeichert. Die erste Auswertungstabelle enthält aus den Einzelbauminformationen hergeleitete Werte auf Artenbasis (zum Beispiel der aktuelle Wert aller Buchen eines Bestandes in Euro pro Hektar). In der zweiten werden auf Bestandesbasis aggregierte Daten abgelegt (zum Beispiel die Summe der Einzelwerte aller Bäume eines Bestandes in Euro pro Hektar).

Zu (2): Für die Fortschreibung jedes Simulationsszenarios wird die Ausgangssituation erneut aus der Ergebnisdatenbank „Status Quo“ eingelesen. Es werden die Tabellen „Einzelbäume“, „Bestände“, „Arten“ und „Verjüngung“ verwendet. Eine Beschreibung der programmiertechnischen Umsetzung der Fortschreibung findet man in Kapitel 3.5.1 für das Szenario LÖWE, in Kapitel 3.5.2 für das Szenario PNV, in Kapitel 3.5.3 für das Szenario ERTRAG und in Kapitel 3.5.4 für das Szenario PROZESS. Die Ergebnisse der Fortschreibung können optional nach jedem Eingriff oder nur zum Ende der Simulation in die entsprechende Ergebnisdatenbank des Szenarios abgelegt werden. Der Benutzer kann wählen, welche Tabellen (Bestandesinformationen, Einzelbauminformationen, Arteninformationen, Verjüngungsinformationen, Auswertung Arten und Auswertung Bestand) er sichern möchte. 


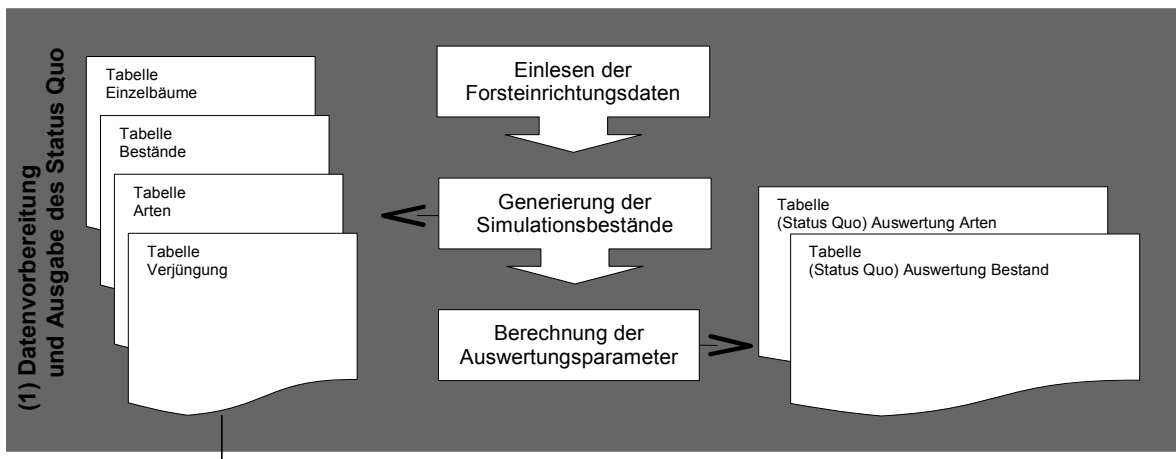

\author{
(3) vergleichende \\ Auswertung
}

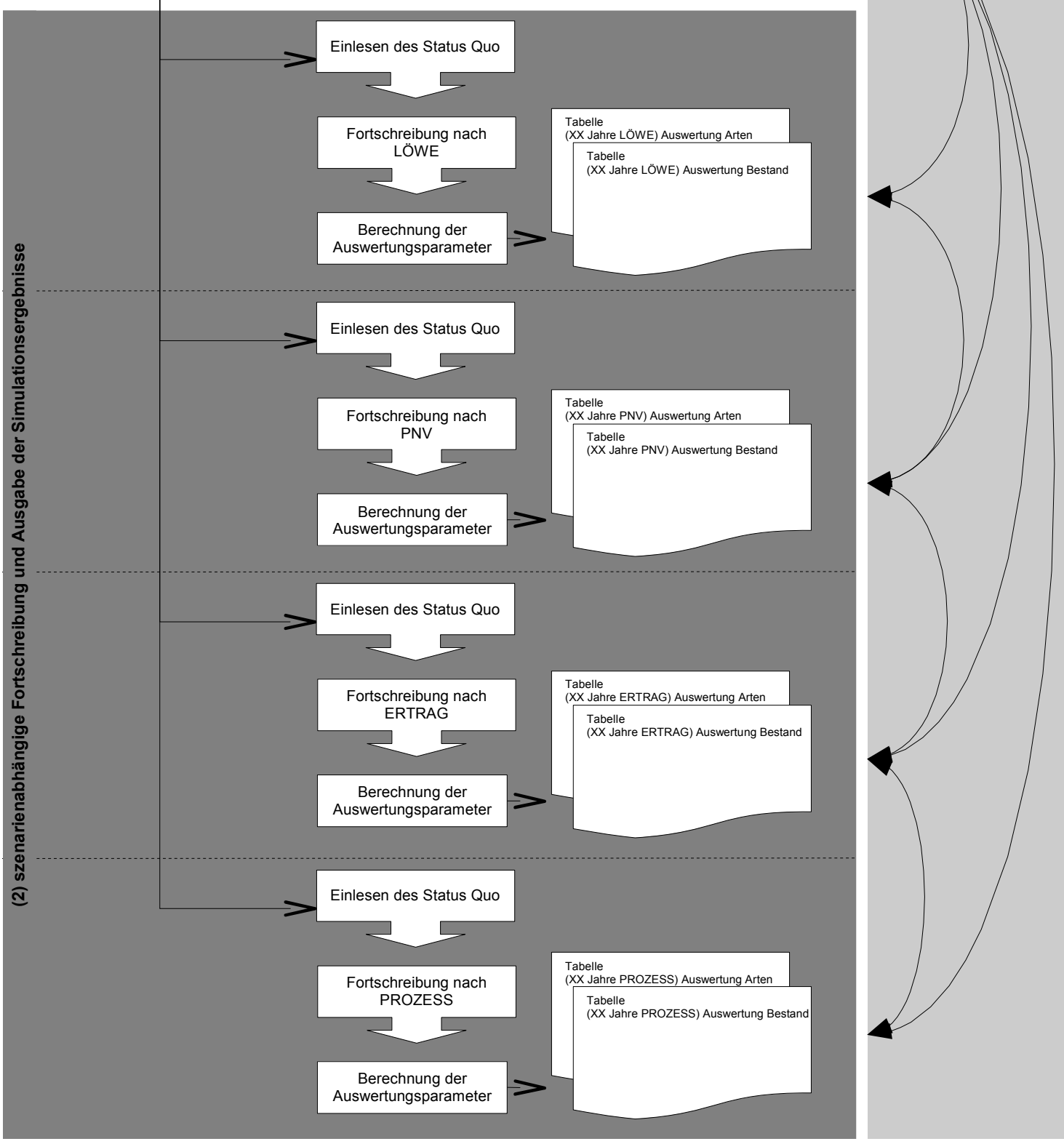

Abbildung 3.3.2.b: Übersicht über die Verarbeitungsschritte: (1) Datenvorbereitung und Ausgabe der Ausgangssituation, (2) szenarienabhängige Fortschreibung und Ausgabe der Simulationsergebnisse und (3) vergleichende Auswertung. 
$\mathrm{Zu}$ beachten ist, daß sich die Laufzeit des Programms mit jeder zusätzlichen Tabelle erhöht. Zur vergleichenden Auswertung werden lediglich die beiden Auswertungstabellen („Auswertung auf Artenebene“ und „Auswertung auf Bestandesebene“) benötigt. Die Tabellen „Einzelbäume“, „Bestände“, „Arten“ und „Verjüngung“ müssen nur gespeichert werden, wenn man eine weitere Simulationsperiode anschließen möchte.

Zu (3): Da Daten zur Ausgangssituation und Daten zu jedem Simulationslauf (in Fünf- oder Zehnjahresschritten) festgehalten werden können, sind die Vergleichsmöglichkeiten groß. Es können Unterschiede der Varianten untereinander, der Unterschied zwischen Anfangsund Endzustand oder auch die Entwicklung eines Parameters über den Simulationszeitraum hinweg ausgewertet werden. Unter Anwendung eines geographischen Informationssystems (GIS) kann diese Auswertung auch mit geographischem Bezug vorgenommen werden.

\subsection{Datenvorbereitung und Ausgabe des Status Quo}

Ein Ziel des ersten Datenverarbeitungsschritts (Datenvorbereitung und Ausgabe der Ausgangssituation, siehe Abbildung 3.3.2.b) ist es, aus verteilungsbezogenen Kennzahlen positionsabhängige Einzelbaumdaten für jeden fortzuschreibenden Bestand auszugeben und mehrfach abrufbar zu speichern (siehe Kapitel 3.4.1). Die folgenden Simulationsläufe können somit auf der exakt gleichen Datenbasis starten. Unterschiede in den Simulationsläufen verschiedener Szenarien können somit nicht auf Zufallseffekte bei der Generierung der Einzelbaumdaten zurückgeführt werden. Solche Zufallseffekte spielen zum Beispiel bei der räumlichen Anordnung der Bäume eine Rolle.

Ein weiteres Ziel des ersten Verarbeitungsschritts ist die Ausgabe von Auswertungskennzahlen der Ausgangssituation (siehe Kapitel 3.4.2), die mit dem Zustand der Bestände nach Ablauf des Simulationszeitraums verglichen werden kann.

\subsubsection{Einlesen der Forsteinrichtungsdaten und Generierung der Simulationsbestände}

Die Einleseroutine ist in der Lage, Forsteinrichtungsdaten aus einer PostgreSQLDatenbank zu verarbeiten. Die Zuordnung der Spalten erfolgt über standardisierte Spaltennamen, die in Tabelle 3.4.1.a zu finden sind. Für jede Unterabteilung (Definition siehe Glossar) liegen im Ausgangsdatensatz Forsteinrichtungsdaten vor. Zu jeder Unterabteilung können mehrere Zeilen Informationen gehören. Jede dieser Zeilen enthält die Verteilungsinformationen eines Baumkollektivs gleicher Art und gleichen Alters. Ein Simulationsbestand wird aus der Summe dieser Verteilungen, also unterabteilungsweise, gebildet. Zwar scheidet die Forsteinrichtung unterhalb der Unterabteilung in manchen Fällen Hilfsflächen (Definition siehe Glossar) aus, da diese jedoch nicht kartographisch festgehalten werden, wäre eine räumliche Auswertung der Ergebnisse nicht möglich. Verteilungsinformationen von Hilfsflächen werden daher beim Bestandesaufbau behandelt, als seien sie Teile der Unterabteilung. Sie werden nicht räumlich von der Unterabteilung getrennt. 
Tabelle 3.4.1.a: Aufbau der Tabelle für Forsteinrichtungsdaten aus der Quelldatenbank.

\begin{tabular}{|c|c|c|c|c|}
\hline $\begin{array}{l}\text { Spalten- } \\
\text { name }\end{array}$ & $\begin{array}{l}\text { direkt } \\
\text { simulations- } \\
\text { relevant }\end{array}$ & Format & Inhalt & Alternative Herleitung \\
\hline$n$ & ja & int4 & Fortlaufende Nummer & nicht möglich \\
\hline$i d$ & ja & text & Bestandesname & nicht möglich \\
\hline species & ja & int4 & Artenkennzahl & nicht möglich \\
\hline age & ja & double & Alter & nicht möglich \\
\hline$d g$ & ja & double & Mitteldurchmesser & aus nicht ersetzbaren Parametern und ycr \\
\hline hg & ja & double & Mittelhöhe & aus nicht ersetzbaren Parametern und ycr \\
\hline$d \max$ & ja & double & maximaler Durchmesser & aus nicht ersetzbaren Parametern \\
\hline ba_ha & ja & double & Grundfläche pro Hektar & $\begin{array}{l}\text { aus standsize, owner, ycr, sdi, layerperc, } \\
\text { size }\end{array}$ \\
\hline si & ja & double & $\begin{array}{l}\text { absolute Bonität } \\
\text { (Höhe im Alter 100) }\end{array}$ & aus nicht ersetzbaren Parametern \\
\hline site_1 & ja & double & $\begin{array}{l}\text { Wasser-/ } \\
\text { Nährstoffhaushaltskennziffer }\end{array}$ & nicht möglich \\
\hline standsize & nein & double & Bestandesgröße & aus layer, size \\
\hline owner & nein & int4 & Besitzerkennzahl & für ba_ha \\
\hline$y c r$ & nein & double & Leistungsklasse & für ba_ha \\
\hline sdi & nein & double & Bestockungsgrad & für ba_ha \\
\hline layer & nein & double & Schicht & für ba_ha \\
\hline $\begin{array}{l}\text { layerper } \\
\text { c }\end{array}$ & nein & double & Flächenprozent & für ba_ha \\
\hline size & nein & double & Standraum & für ba_ha \\
\hline
\end{tabular}

Die erzeugten Simulationsbestände (entsprechen Unterabteilungen) haben eine Standardgröße von 0,25ha. Von der Beibehaltung der realen Größen der Unterabteilungen wurde abgesehen, da bei einer zu hohen Anzahl von Einzelbäumen die Speicherkapazität des Rechners überschritten wird. Die im Programm festgeschriebene höchste Anzahl ${ }^{12}$ von Bäumen des Bestandes wurde daher auf 4000 Bäume gesetzt. Bei einem Simulationszeitraum von vierzig Jahren wird diese Grenze leicht überschritten, wenn mit realen Größen gearbeitet wird. Ein weiterer Grund für die Einschränkung ist, daß der Rechenaufwand der Simulation überproportional mit dem Anstieg der simulierten Bestandesgröße wächst. Eine 0,25ha große Simulationsfläche ermöglicht die wirklichkeitsnahe Abbildung selbst artenreicher Bestände und senkt das Risiko, daß die Baumzahl auf über 4000 Individuen steigt, auf ein Minimum herab.

Die Zugehörigkeit zu einer Unterabteilung (zu einem Simulationsbestand) muß im Ausgangsdatensatz durch einen eindeutigen Namen gekennzeichnet sein (Spalte „id“, siehe Tabelle 3.4.1.a). Die fortlaufende Nummer (Spalte „n“, siehe Tabelle 3.4.1.a)

12 Diese Anzahl wird als Summe aller seit Simulationsbeginn generierten Individuen des betrachteten Bestandes verstanden. Abgestorbene/entnommene Bäume werden bei dieser Anzahl mitgerechnet. 
ermöglicht es, an beliebiger Stelle der Forsteinrichtungstabelle mit der Generierung der Bestände einzusetzen. Zunächst wird die Summe der Zeilen, die zu der betrachteten Unterabteilung (entspricht dem betrachteten Bestand) gehören, eingelesen. Erst dann beginnt die Datenergänzung.

Das Ziel der Datenergänzung ist es, alle zur Generierung der Einzelbaumdaten nötigen Informationen aus Werten heraus zu ergänzen, die in gängigen Forsteinrichtungsverfahren erhoben werden. Nach der Datenergänzung kann die in BWINPro implementierte Routine Einzelbäume aus den Verteilungsinformationen unter der Voraussetzung generieren, daß das beschriebene Kollektiv als Teil des Bestandes und nicht als Teil der Verjüngung anzusehen ist. Die Grenze wird dabei bei einem Mindest-Dg von $8 \mathrm{~cm}$ und einer Mindesthöhe von 1,3 Metern gezogen. Erreicht das betrachtete Kollektiv diese Grenze nicht, so werden die Angaben als Verjüngungsinformationen gespeichert, so daß im Laufe der Simulation Einwuchs aus diesen Verjüngungsdaten generiert werden kann.

Zur Generierung von Einzelbauminformationen müssen folgende Verteilungskennwerte vorliegen (siehe NAGEL 1999, Definition der Kennwerte siehe Glossar): Art, Alter, Dg, Hg, Dmax, Grundfläche, absolute Bonität (Höhe im Alter 100). In den folgenden Abschnitten wird auf die Möglichkeiten eingegangen, die das Programm zur Herleitung dieser Informationen bietet.

\section{Art}

Die Baumart wird den generierten Einzelbäumen durch eine Artennummer eindeutig zugeordnet. Die verwendete Numerierung ist in der Anweisung zur Betriebsregelung in den Niedersächsischen Landesforsten (B.A.87) zu finden (siehe NiEDERSÄCHSISCHE LANDESFORSTVERWALtUng 1987, Seiten 1A und 2A). Diese Information ist nicht ersetzbar (Spalte „species“, siehe Tabelle 3.4.1.a). Verteilungen, die ungültige Baumartennummern enthalten, können nicht generiert werden.

\section{Alter}

Das Durchschnittsalter einer Schicht (in Jahren) wird als Alter der generierten Einzelbäume übernommen. Auch diese Information muß in jedem Fall vorliegen (Spalte „age“, siehe Tabelle 3.4.1.a). Daten zur Altersspanne, die ein Kollektiv weiter beschreiben, können bei der Generierung der Einzelbäume nicht verarbeitet werden.

\section{$D g$}

Für die Einzelbaumgeneration muß jedem Kollektiv ein Mitteldurchmesser (Dg) zugeordnet sein. Die Datenergänzungsroutinen sind allerdings in der Lage, die Werte herzuleiten, falls sie in den Quelldaten fehlen (Spalte „dg“, siehe Tabelle 3.4.1.a). Die Datenergänzung stützt sich auf Hilfstafeln (NiedersäChSISCHEs ForstPlanungsamt 1987), die eine Beziehung zwischen Baumart, Alter, Leistungsklasse und Dg herstellen. Sind Baumart, Alter und Leistungsklasse bekannt, kann über diese Tafeln ein entsprechender Dg zugeordnet werden. Wird der Dg einer Baumart gesucht, die nicht Bestandteil der Tafeln ist, so wird der Dg einer ähnlich wachsenden Baumart angenommen (zum Beispiel gemeine Fichte 
(Picea abies) statt Blau-Fichte (Picea pungens)). Liegt die Leistungsklasse oder das Alter außerhalb des vorgesehenen Wertebereichs, so wird lediglich zwischen Laubholz (das dann wie Buche behandelt wird) und Nadelholz (das dann wie Fichte behandelt wird) unterschieden. Ist auch der Umfang dieser Tafeln nicht ausreichend, wird der gesuchte Wert durch Extrapolation ermittelt.

\section{$H g$}

Fehlende Hg-Werte (Spalte „hg“, siehe Tabelle 3.4.1.a) werden analog zu dem Verfahren bei fehlendem Dg ergänzt. Mit Hilfe von Baumart, Alter und Leistungsklasse wird durch den Einsatz von Hilfstafeln (nach Niedersächsisches ForstPlanungsamt 1987) auf die Hg geschlossen. In dem Fall, daß das Alter der Verteilung unter dem niedrigsten in der Hilfstafel vorkommenden Alter liegt, wird eine einfache lineare Beziehung zwischen Dg (der gegebenenfalls im Schritt zuvor ergänzt wurde) und Höhe angenommen.

\section{Dmax}

Der Dmax wird mit Hilfe zweier einfacher Beziehungen aus dem Dg der Verteilung geschätzt. Ist der Dg geringer als $15 \mathrm{~cm}$, so wird angenommen, daß der dazugehörige Dmax beim 1,1-fachen dieses Werts liegt. Bei einem Dg von $15 \mathrm{~cm}$ oder darüber wird der Dmax auf das 1,4-fache geschätzt.

\section{Grundfläche}

Die Anzahl der zu generierenden Einzelbäume wird durch die von der Art eingenommene Grundfläche im Bestand gesteuert. Ist die angegebene Grundfläche erreicht, bricht die Generierungsroutine $a b$. Die zu generierende Grundfläche wird in mehreren Schritten hergeleitet. Zunächst wird die Grundfläche pro Hektar ermittelt, die bei der vorliegenden Baumart, der gegebenen Leistungsklasse und dem angegebenen Alter in einem Bestand mit Bestockungsgrad 1 zu erwarten ist (Spalte „ycr“, siehe Tabelle 3.4.1.a). Dazu wird auf eine Hilfstafel (nach Niedersächsisches ForstPlanungsamt 1987) zurückgegriffen. Analog zum Vorgehen bei $\mathrm{Hg}$ und $\mathrm{Dg}$ ist auch diese Routine in der Lage, die Grundfläche durch Extrapolation zu ermitteln, falls die gegebenen Werte (Alter oder Leistungsklasse) nicht im Bereich der Tafel liegen. Die so ermittelte Grundfläche pro Hektar muß nun noch mit dem angegebenen Bestockungsgrad multipliziert werden. Dieser wird der Forsteinrichtungstabelle entnommen (Spalte „sdi“, siehe Tabelle 3.4.1.a). Die tatsächliche Grundfläche pro Hektar ergibt sich aus der Multiplikation des Werts pro Hektar mit der Anteilfläche, die die Baumart pro Hektar einnimmt. Dazu wird das Verhältnis des tatsächlichen Standraums der Baumart zur tatsächlichen Bestandesfläche verlangt.

Je nach der Herkunft der Ausgangsdaten ist dieser Prozentsatz direkt bekannt oder muß erst noch ermittelt werden. Welcher Rechenweg verwendet wird, hängt von der Angabe in der Spalte „owner" ab. Bei den Ausgangsdaten, die von der Forstbetriebsgemeinschaft („owner“ = 2) aufgenommen wurden, entsprechen die Werte der Spalte („layerperc“, siehe Tabelle 3.4.1.a) schon dem gesuchten Flächenanteil. 
Für Forsteinrichtungsdaten, die ähnlich zu dem Verfahren der Niedersächsischen Landesforsten erhoben wurden - dazu zählen in dieser Arbeit die Niedersächsischen Landesforsten selbst („owner" = 1) und das Forstamt der Klosterkammer Soltau („owner" = 3) -, wird folgende Berechnung angewendet: Zunächst wird die Fläche errechnet, auf der die Baumart stockt. Dazu werden die Standräume aller Kollektive (Spalte „size“, siehe Tabelle 3.4.1.a), die einer Schicht und derselben Teilfläche (Hilfsfläche oder restliche Bestandesfläche) angehören, aufsummiert. Da in der Forsteinrichtung ab 0,05ha abgerundet wird, kann die Summe der Standräume 0 sein. In diesem Fall wird den Zeilen dann eine Zufallszahl zwischen 0,001 und 0,05 zugeordnet. Über den Prozentsatz, den die Teilkollektive an der betrachteten Schicht der betrachteten Teilfläche haben („layerperc“, siehe Tabelle 3.4.1.a), der Summe des Standraumes der Schicht in der Teilfläche und der Gesamtbestandesgröße kann dann der prozentuale Anteil des Teilkollektivs (einer Zeile) am Gesamtbestand errechnet werden. Die Bestandesgröße selbst kann entweder aus der Datengrundlage übernommen werden („standsize“) oder aus den Spalten „size“ und „layer" hergeleitet werden (siehe Tabelle 3.4.1.a).

\section{Absolute Bonität}

Die Höhe im Alter 100 (absolute Bonität) steuert neben der Art und Konkurrenzfaktoren das Höhenwachstum eines Baums. Der Wachstumssimulator BWINPro ist in der Lage, eine solche Angabe aus der $\mathrm{H} 100$ und dem dazugehörigen Alter artengetrennt zuzuordnen. Diese Funktion wird von den im Rahmen dieser Arbeit erstellten Komponenten genutzt. Dabei wird immer auf die Kennzahlen des ältesten Kollektivs einer Art zurückgegriffen. Ist das betrachtete Kollektiv besonders alt (über 100 Jahre) oder besonders jung (es gehört der Verjüngung an), wird statt auf diese BWIN-Funktion auf die Hilfstafeln der Metadatenbank (nach Niedersächsisches ForstPlanungsamt 1987) zurückgegriffen, aus der mit Hilfe der Leistungsklasse und der Baumart die im Alter $100 \mathrm{zu}$ erwartende Höhe ermittelt wird.

\subsubsection{Bestimmung der Zieltypen und Berechnung der Auswertungsparameter}

Nach der Datenaufbereitung werden die in Kapitel 3.6 beschriebenen Parameter berechnet und ausgegeben. Dabei beschränkt sich die Ausgabe auf Werte, die nicht erst nach dem ersten Fortschreibungsschritt errechnet werden können (siehe Tabelle 3.6.2.a, letzte Spalte). Es entfallen zum Beispiel alle zuwachs- und nutzungsabhängigen Werte.

Vor der Berechnung der Zielerreichungsindizes (siehe Tabelle 3.6.2.a) werden den Beständen szenarienabhängige Zieltypen zugeordnet. Ein Zieltyp definiert sich über seine Haupt- und Nebenbaumarten und über die Mischungsanteile, die Haupt-, Neben- und Begleitbaumarten einnehmen sollen. Der Schritt der Zuordnung ist für den weiteren Verlauf der Simulation entscheidend, denn die hier festgelegten Zieltypen werden bis zum Ende der Simulation beibehalten. Ihre Festlegung wird der eigentlichen Fortschreibung vorweggenommen, da so schon für den Status Quo angegeben werden kann, wie sehr sich die angestrebten Artenzusammensetzungen von der Artenzusammensetzung zum Zeitpunkt 0 unterscheiden. Außerdem enthält die Auswahl der Zieltypen zufallsgesteuerte 
Elemente. Durch Speicherung der Zieltypen mit dem Status Quo kann sichergestellt werden, daß bei mehreren Simulationsläufen immer auf dieselbe Baumartenmischung hingearbeitet wird.

\section{Zuordnung der Zieltypen für das Szenario LÖWE}

Die Zieltypen des Szenarios LÖWE sind eng an die Richtlinie zur Baumartenwahl (Niedersächsische LandesforstVerWAltung 2004) gekoppelt. Die dort festgehaltenen Waldentwicklungstypen (WET) beschreiben Leitbilder des angestrebten Waldaufbaus. Die Leitbilder charakterisieren die sukzessionale Stellung des WET und nennen Entwicklungsziele hinsichtlich der Holzerzeugung, der Schutz- und Erholungsfunktionen sowie der Baumartenanteile. Die LÖWE-Zieltypen übernehmen die in den WETs vorgegebenen Baumarten und lehnen sich an die vorgegebenen Mischungsanteile an. Ihre Zuordnung geschieht analog zu der WET-Zuordnung, die in der Richtlinie beschrieben wird. Sie ist abhängig von der betrachteten Waldbauregion, der waldbaulichen Ausgangssituation, den Standortseigenschaften (Nährstoffversorgung, Wasserversorgung) und der Rangfolge der in Frage kommenden WET-Typen.

Programmiertechnisch ist die Zuordnung wie folgt gelöst: Zunächst wird über die den Unterabteilungen zugeordnete Nährstoff- und Wasserversorgungskennzahl (siehe Tabelle 3.4.1.a, Spalte „site_1“) auf die möglichen vorrangigen WET-Typen geschlossen. Dazu wird die Tabelle "Zuordnung Zieltypen LÖWE“ der Metadatenbank genutzt. Diese entspricht exakt den WET-Zuordnungstabellen der für das Untersuchungsgebiet relevanten Waldbauregionen 6 und 7 (NiedeRsächsische LANDESFORSTVERWALTUNG 2004, S. 38 und S. 43). Von den in Frage kommenden Typen werden jene ausgeschlossen, die aus der aktuellen Baumartenzusammensetzung heraus nicht in Frage kommen. Die Beschränkungen wurden den verbal formulierten Entscheidungskriterien der Richtlinie zur Baumartenwahl (Niedersächsische LANDESFORStVerwaltung 2004, S. 6) entnommen. Beispielsweise müssen Eichenbestandestypen in Eichen-WETs und BuchenBestandestypen in Buchen-WETs übergehen. Aus den verbliebenen Möglichkeiten wird durch eine zufallsgesteuerte Entscheidung ein WET ausgewählt. Die Auswahlwahrscheinlichkeiten richten sich nach der Rangfolge der WETs. So wird zum Beispiel bei drei in Frage kommenden WETs der erste mit einer Wahrscheinlichkeit von $50 \%$, der zweite mit einer Wahrscheinlichkeit von $30 \%$ und der dritte mit einer Wahrscheinlichkeit von 20\% gewählt (die verwendete Gewichtung ist auf Seite 13 der Richtlinie zu finden). Kommt keiner der vorrangigen WETs für die Entwicklung des Bestandes in Frage, so wird mit dem gleichen Verfahren geprüft, ob ein nachrangiger WET gewählt werden kann. Sind auch alle nachrangigen WETs ausgeschlossen, so geht die Routine davon aus, daß die Fehlbestockung des Bestandes langfristig in einen der für diesen Standort vorrangigen WETs umgebaut werden soll. Auch diese Wahl fällt zufallsgesteuert und berücksichtigt die Auswahlwahrscheinlichkeiten.

Abhängig vom ausgewählten Zieltypen können nun mit Hilfe der Tabelle „Zuordnung Baumarten" der Metadatenbank den Baumarten des Bestandes und der Verjüngung ein Status als Hauptbaumart, Nebenbaumart oder nicht vorgesehener Baumart zugeordnet werden. Den gewünschten Haupt- und Nebenbaumarten wird ein angestrebter Mischungsanteil zugeordnet. Der anzustrebende Mischungsanteil von Haupt- und 
Nebenbaumarten eines WETs ist in der Tabelle „Zuordnung Prozente“ der Metadatenbank zu finden. Er lehnt sich an die in der Richtlinie zur Baumartenwahl angegebenen Werte zu dem jeweiligen WET an und ist als Anteil der Baumart an der Summe der Kronenschirmflächen des Bestandes zu verstehen. Da in der Richtlinie oft Prozentspannen angegeben sind, wurden die Maximal- und Minimalwerte gemittelt. Gibt es zwei mögliche Hauptbaumarten, wie zum Beispiel im Edellaubbaum-WET 31 (Esche/ Bergahorn) der Fall, so richtet sich die angestrebte prozentuale Verteilung nach dem aktuellen Verhältnis der beiden Arten im Bestand.

\section{Zuordnung der Zieltypen für das Szenario PNV}

Der anzustrebende PNV-Typ wurde den Beständen über eine GIS-gesteuerte Flächenzuordnung im vorhinein aus der pnV-Karte nach Kaiser und Zacharias (KAISER UND ZACHARIAS 2003) zugeordnet. Kommen für einen Bestand mehrere PNV-Typen in Frage, so wurde derjenige mit dem größten Flächenanteil am Bestand gewählt. In der Metadatenbank ist das Ergebnis der Zuordnung in der Tabelle „Zuordnung Zieltypen PNV“ zu finden. Analog zum Vorgehen beim Zuordnen der LÖWE-Zieltypen sind auch für die PNV-Typen in der Tabelle „Zuordnung Baumarten“ die Haupt- und Nebenbaumarten eines PNV-Zieltyps zu finden. Die Tabelle setzt die PNV-Typenbeschreibung um, die in der Veröffentlichung KAISER UND ZACHARIAS 2003 auf den Seiten 8 und 9 zu finden ist. Sind für einen PNV-Typ verschiedene Baumartenmischungen möglich, kann dem Bestand eine der Möglichkeiten zufällig zugeordnet werden. Die möglichen PNV-Typen können mit gleicher Wahrscheinlichkeit gewählt werden. In der Tabelle „Zuordnung Prozente“ der Metadatenbank wird der Hauptbaumart eines PNV-Zieltyps standardmäßig ein Anteil von $70 \%$, der Nebenbaumart ein Anteil von $20 \%$ zugewiesen.

\section{Zuordnung der Zieltypen für das Szenario ERTRAG}

Im Untersuchungsgebiet sollen in erster Linie die dort dominierenden Kiefernwälder in Douglasien- oder Douglasien-/ Buchenbestände überführt werden. Als Entwicklungsziel werden den Beständen vorwiegend Reinbestandestypen der Baumarten zugeordnet, die nach der gegebenen standörtlichen Ausstattung den höchsten Ertrag versprechen. Dabei wird häufig auf die Baumart Douglasie gesetzt. Programmiertechnisch wird analog zur Zieltypenzuordnung für LÖWE gearbeitet, nur die Zuordnung des Zieltyps zum Standort wurde modifiziert. In der Tabelle „Zieltypen Ertrag“ wurden gutachterlich den Standortstypen überall dort Reinbestände ertragsstarker Baumarten (Douglasie, Fichte und Buche) zugeordnet, wo sie waldbaulich vertretbar sind. Bei Standorten, die eine Nährstoffversorgung besser oder gleich 4 aufweisen, kommen zusätzlich Eichenreinbestände in Frage. Auf ertragsschwachen Standorten oder Sonderstandorten wurde die Zuordnung der LÖWE-WETs übernommen. Gemäß der Nährstoff- und Wasserkennziffer eines Bestandes werden mögliche Zieltypen zugeordnet. Dabei werden zunächst alle Typen ausgeschlossen, bei denen große Umbaumaßnahmen nötig würden. Dieses Vorgehen verhindert hohe Investitionen in den Umbau eines Bestandes, wenn bereits eine Bestockung mit angemessener Ertragserwartung vorliegt. Sind für alle Zieltypen Umbaumaßnahmen notwendig, so wird einer der ERTRAG-Zieltypen für diesen Standort zufallsgesteuert ausgewählt. Die Variante ERTRAG kennt keine nachrangigen 
Zieltypen. Die Benennung von Haupt- und Nebenbaumarten geschieht auch in dieser Variante über die Angaben der Tabelle „Zuordnung Baumarten“ der Metadatenbank. In Reinbeständen gibt es definitionsgemäß keine Nebenbaumarten, der Hauptbaumart wird ein angestrebter Anteil von $100 \%$ zugeordnet.

\section{Zuordnung der Zieltypen für das Szenario PROZESS}

Für das Szenario PROZESS werden keine Zieltypen festgelegt. Die Baumartenmischung soll in diesem Konzept nicht durch Eingriffe beeinflußt werden. Vielmehr sollen sich die konkurrenzstärksten Baumarten im Bestand durchsetzen (siehe Kapitel 3.1.4).

\subsection{Szenarienabhängige Fortschreibung und Ausgabe der Simulationsergebnisse}

Nach der Aufbereitung der Daten schließen sich vier szenarienabhängige Behandlungszyklen an (siehe Abbildung 3.5.a). Die Behandlungszyklen bestehen aus Einlesen, Fortschreibezyklus und Ausgabe. Sie arbeiten bestandesweise, das heißt die Bestände des Status Quo werden nacheinander eingelesen, fortgeschrieben und ausgegeben. Das Fortschreiben gliedert sich in fünf Schritte, auf die im einzelnen später in diesem Kapitel eingegangen wird. Es startet vor der ersten Wachstumsperiode mit dem Setzen der Rahmenwerte und einem ersten Eingriff, da die Forsteinrichtungsdaten keinerlei Informationen enthalten, wann der letzte Eingriff in einem Bestand durchgeführt wurde. Entsprechend dem 40jährigen Simulationszeitraum sind 8 fünfjährige Wachstumsperioden vorgesehen. Der Simulationszeitraum kann gegebenenfalls angepaßt werden. Ob und welcher Eingriff zum Beginn der Simulation oder nach einer Wachstumsperiode stattfindet, hängt von der aktuellen Bestandessituation und den gesetzten Rahmenwerten ab. Nach jeder Wachstumsperiode wird die Gültigkeit der Rahmenwerte überprüft und sie werden gegebenenfalls neu gesetzt. Nach jedem Eingriff besteht die Möglichkeit der Datenausgabe. Nach der vierzigjährigen Fortschreibungsperiode wird der Fortschreibezyklus mit der Ausgabe der Auswertungsparameter beendet und der nächste Bestand des Untersuchungsgebiets wird eingelesen. Sind alle Bestände des Untersuchungsgebiets fortgeschrieben, so ist der Behandlungszyklus für ein Szenario abgeschlossen.

Die programmiertechnische Umsetzung verschiedener Waldbaukonzepte wird durch szenarienabhängige Rahmenwerte, eine szenarienabhängige Auswahl an Maßnahmenelementen und einer szenarienabhägigen Verkettung von Maßnahmenelementen ermöglicht. Die Maßnahmenelemente lassen sich gemäß den Schritten des Fortschreibezyklus einem der folgenden Typen zuordnen: Schutz, Endnutzung, Durchforstung oder Verjüngung. Ein Schritt des Fortschreibezyklus kann sich in mehrere Maßnahmenelemente gliedern. Ein und dasselbe Maßnahmenelement kann Teil mehrerer Waldbaukonzepte sein. Im Fortschreibungszyklus werden die Maßnahmenelemente in eine Reihenfolge (Maßnahmenkette) gebracht, die die Priorität ihrer Anwendung zum Ausdruck bringt. Nicht alle Maßnahmenelemente werden zwangsläufig in jedem Fortschreibungszyklus ausgeführt. 


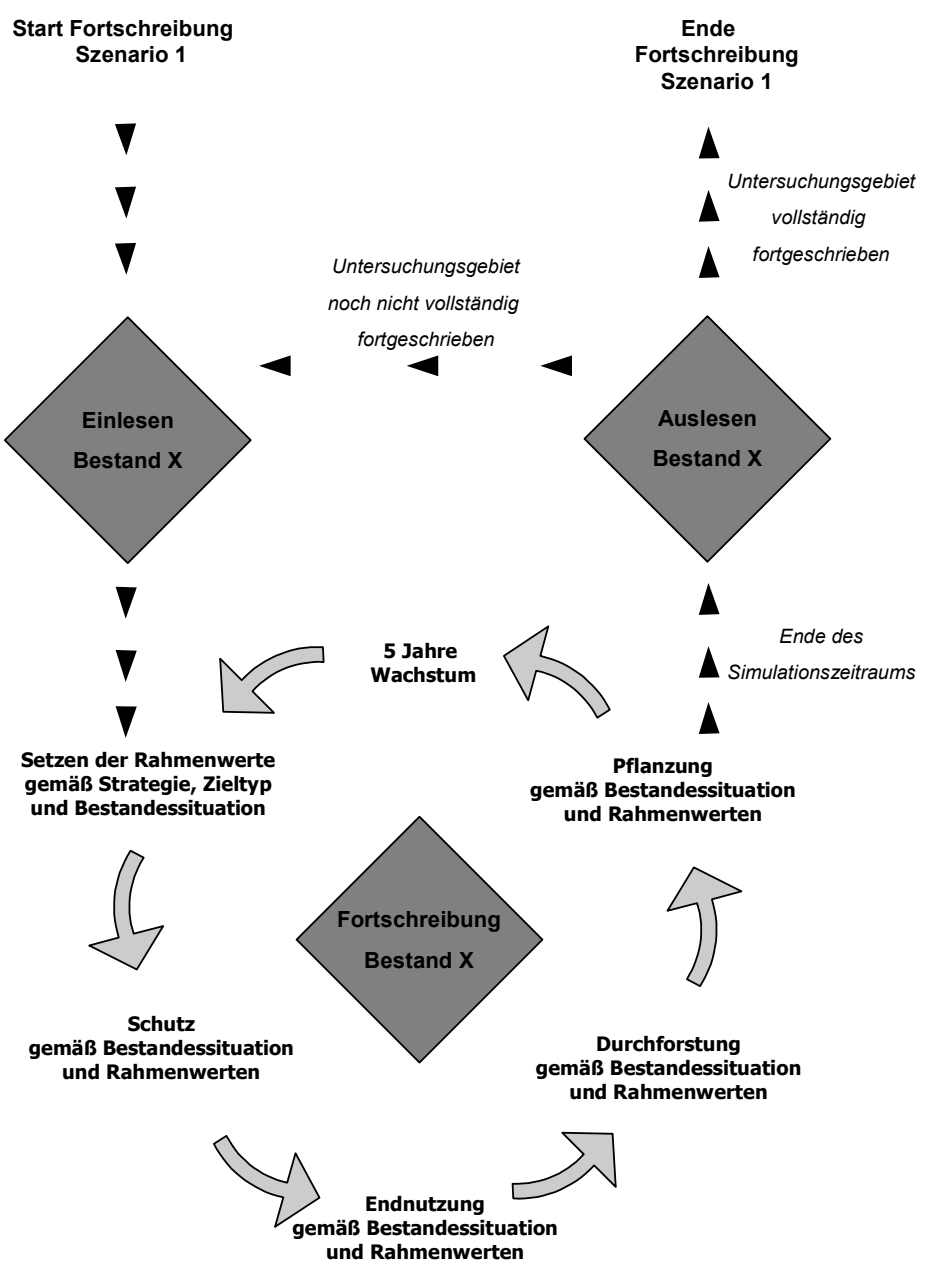

Abbildung 3.5.a: Ablauf der szenarienabhängigen Fortschreibung des Untersuchungsgebiets.

Im folgenden wird auf die Inhalte der fünf Schritte des Fortschreibungszyklus eingegangen. Es werden die szenarienabhängigen Rahmenwerte (siehe auch Tabellen 3.5.a und 3.5.b) und verwendete Schutz-, Endnutzungs-, Durchforstungs- und Verjüngungselemente (siehe Tabelle 3.5.c) vorgestellt. Die szenarienabhängige Wahl der Rahmenwerte und die Zusammensetzung der Maßnahmenketten werden in den Kapiteln 3.5.1 (für LÖWE), 3.5.2 (für PNV), 3.5.3 (für ERTRAG) und 3.5.4 (für PROZESS) erläutert.

\section{Rahmenwerte}

Die Rahmenwerte steuern, welche Eingriffe wie stattfinden, und beeinflussen somit den gesamten Simulationsablauf der Waldbaustrategie. Ob ein Maßnahmenelement, das Teil einer Waldbaustrategie ist, zum Einsatz kommt und wie es parametrisiert wird, hängt von der aktuellen Bestandessituation und den szenarienabhängigen Rahmenwerten ab. Es werden Rahmenwerte auf Bestandesebene von Rahmenwerten auf Artenebene unterschieden. Die festgelegten Werte können für die vier Strategien den Tabellen 3.5.a und 3.5.b entnommen werden. 
Tabelle 3.5.a: Rahmenwerte (bestandesbezogene Werte) zu den Strategien LÖWE, PNV, ERTRAG und PROZESS.

\begin{tabular}{|c|c|c|c|c|c|}
\hline $\begin{array}{l}\text { Rahmenwerte Bestand } \\
\text { (Einheit) }\end{array}$ & Bestandestyp & $L \ddot{O} W E$ & $P N V$ & ERTRAG & PROZESS \\
\hline $\begin{array}{l}\text { Eingriffsturnus } \\
\text { (Jahre) }\end{array}$ & alle & 5 & 5 & 10 & 10 \\
\hline \multirow{8}{*}{$\begin{array}{l}\text { Maximales Endnutzungsvolumen } \\
\text { (Vorratsfestmeter je Hektar) }\end{array}$} & Eiche & 100 & 100 & 400 & 200 \\
\hline & Buche & 100 & 100 & 160 & 100 \\
\hline & Alh & 80 & 80 & 160 & 80 \\
\hline & Aln & 80 & 80 & 200 & 80 \\
\hline & Fichte & 100 & 100 & 200 & 100 \\
\hline & Douglasie & 100 & 100 & 200 & 100 \\
\hline & Kiefer & 70 & 70 & 150 & 70 \\
\hline & Lärche & 70 & 70 & 150 & 70 \\
\hline \multirow{8}{*}{$\begin{array}{l}\text { Minimales Endnutzungsvolumen } \\
\text { (Vorratsfestmeter je Hektar) }\end{array}$} & Eiche & 50 & 50 & 200 & 50 \\
\hline & Buche & 40 & 40 & 80 & 40 \\
\hline & Alh & 40 & 40 & 80 & 40 \\
\hline & Aln & 40 & 40 & 100 & 40 \\
\hline & Fichte & 50 & 50 & 100 & 50 \\
\hline & Douglasie & 50 & 50 & 100 & 50 \\
\hline & Kiefer & 35 & 35 & 75 & 35 \\
\hline & Lärche & 35 & 35 & 75 & 35 \\
\hline \multirow{8}{*}{$\begin{array}{l}\text { Maximales } \\
\text { Durchforstungsvolumen } \\
\text { (Vorratsfestmeter je Hektar) }\end{array}$} & Eiche & 70 & 70 & 100 & 70 \\
\hline & Buche & 70 & 70 & 140 & 70 \\
\hline & Alh & 70 & 70 & 140 & 70 \\
\hline & Aln & 70 & 70 & 140 & 70 \\
\hline & Fichte & 70 & 70 & 140 & 70 \\
\hline & Douglasie & 70 & 70 & 140 & 70 \\
\hline & Kiefer & 70 & 70 & 100 & 70 \\
\hline & Lärche & 70 & 70 & 140 & 70 \\
\hline \multirow{8}{*}{$\begin{array}{l}\text { Minimales } \\
\text { Durchforstungsvolumen } \\
\text { (Vorratsfestmeter je Hektar) }\end{array}$} & Eiche & 20 & 20 & 50 & 20 \\
\hline & Buche & 20 & 20 & 70 & 20 \\
\hline & Alh & 20 & 20 & 70 & 20 \\
\hline & Aln & 20 & 20 & 70 & 20 \\
\hline & Fichte & 20 & 20 & 70 & 20 \\
\hline & Douglasie & 20 & 20 & 70 & 20 \\
\hline & Kiefer & 20 & 20 & 50 & 20 \\
\hline & Lärche & 20 & 20 & 70 & 20 \\
\hline $\begin{array}{l}\text { Maximales Eingriffsvolumen } \\
\text { (Vorratsfestmeter je Hektar) }\end{array}$ & alle & \multicolumn{4}{|c|}{$\begin{array}{l}\text { Maximales Durchforstungsvolumen oder } \\
\text { Endnutzungsvolumen (der größere Wert) }\end{array}$} \\
\hline $\begin{array}{l}\text { Minimales Eingriffsvolumen } \\
\text { (Vorratsfestmeter je Hektar) }\end{array}$ & alle & \multicolumn{4}{|c|}{$\begin{array}{l}\text { Minimales Durchforstungsvolumen oder } \\
\text { Endnutzungsvolumen (der kleinere Wert) }\end{array}$} \\
\hline \multirow{8}{*}{$\begin{array}{l}\text { Endnutzungszeitraum } \\
\text { (Jahre) }\end{array}$} & Eiche & $\infty$ & $\infty$ & 10 & $\infty$ \\
\hline & Buche & $\infty$ & $\infty$ & 30 & $\infty$ \\
\hline & Alh & $\infty$ & $\infty$ & 30 & $\infty$ \\
\hline & $A \ln$ & $\infty$ & $\infty$ & 10 & $\infty$ \\
\hline & Fichte & $\infty$ & $\infty$ & 20 & $\infty$ \\
\hline & Douglasie & $\infty$ & $\infty$ & 20 & $\infty$ \\
\hline & Kiefer & $\infty$ & $\infty$ & 20 & $\infty$ \\
\hline & Lärche & $\infty$ & $\infty$ & 20 & $\infty$ \\
\hline $\begin{array}{l}\text { Anzahl Habitatbäume } \\
\text { (n) }\end{array}$ & alle & 1 & 0 & 0 & 1 \\
\hline $\begin{array}{l}\text { Schutz von Minderheiten } \\
\text { (ja/nein) }\end{array}$ & alle & ja & nein & nein & nein \\
\hline $\begin{array}{l}\text { Angestrebter Zieltyp } \\
\text { (keine Einheit) }\end{array}$ & alle & $\begin{array}{l}\text { abhängi } \\
\text { Standor } \\
\text { Bestand }\end{array}$ & $\begin{array}{l}\text { abhän } \\
\text { der pn } \\
\text { Karte }\end{array}$ & $\begin{array}{l}\text { abhängig von } \\
\text { Standort und } \\
\text { Bestandestyp }\end{array}$ & keiner \\
\hline
\end{tabular}


Tabelle 3.5.b: Rahmenwerte (artenbezogene Werte) zu den Strategien LÖWE, PNV, ERTRAG und PROZESS.

\begin{tabular}{|c|c|c|c|c|c|}
\hline $\begin{array}{l}\text { Rahmenwerte Baumarten } \\
\text { (Einheit) }\end{array}$ & $\begin{array}{l}\text { Baumarten- } \\
\text { gruppe }\end{array}$ & $L \ddot{O} W E$ & $P N V$ & ERTRAG & PROZESS \\
\hline \multirow{8}{*}{$\begin{array}{l}\text { Mindesthöhe für ersten Eingriff } \\
\text { (m) }\end{array}$} & Eiche & 14 & 14 & 16 & 16 \\
\hline & Buche & 16 & 16 & 18 & 18 \\
\hline & Alh & 12 & 12 & 14 & 14 \\
\hline & Aln & 10 & 10 & 12 & 12 \\
\hline & Fichte & 14 & 14 & 16 & 16 \\
\hline & Douglasie & 14 & 14 & 16 & 16 \\
\hline & Kiefer & 13 & 13 & 16 & 16 \\
\hline & Lärche & 12 & 12 & 14 & 14 \\
\hline \multirow{9}{*}{$\begin{array}{l}\text { Zielstärke bzw. Ziel-Dg für die } \\
\text { Variante ERTRAG } \\
\text { (cm) }\end{array}$} & Eiche & 70 & 70 & 60 & 70 \\
\hline & Buche & 60 & 60 & 50 & 60 \\
\hline & Hainbuche & 45 & 45 & 45 & 45 \\
\hline & Alh & 60 & 60 & 60 & 60 \\
\hline & Aln & 45 & 45 & 40 & 45 \\
\hline & Fichte & 45 & 45 & 40 & 45 \\
\hline & Douglasie & 60 & 60 & 50 & 60 \\
\hline & Kiefer & 45 & 45 & 40 & 45 \\
\hline & Lärche & 60 & 60 & 50 & 60 \\
\hline \multirow{9}{*}{$\begin{array}{l}\text { Freistellungsgrad } \\
\text { (keine Einheit) }\end{array}$} & allgemein & normal & stark & stark & gering \\
\hline & Eiche & $-0,1$ & 0 & 0 & $-0,15$ \\
\hline & Buche & $-0,3$ & $-0,2$ & $-0,2$ & $-0,35$ \\
\hline & Alh & $-0,2$ & $-0,1$ & $-0,1$ & $-0,25$ \\
\hline & Aln & $-0,1$ & 0 & 0 & $-0,15$ \\
\hline & Fichte & $-0,2$ & $-0,1$ & $-0,1$ & $-0,35$ \\
\hline & Douglasie & $-0,3$ & $-0,2$ & $-0,2$ & $-0,35$ \\
\hline & Kiefer & $-0,1$ & 0 & 0 & $-0,15$ \\
\hline & Lärche & $-0,1$ & 0 & 0 & $-0,15$ \\
\hline $\begin{array}{l}\text { Ziel-Artenanteil } \\
\text { (\% der Kronenschirmfläche) }\end{array}$ & alle & Laut Zieltyp & Laut Zieltyp & Laut Zieltyp & $\begin{array}{l}\text { keine } \\
\text { Vorgabe }\end{array}$ \\
\hline
\end{tabular}

Ein Beispiel soll den Einsatz von Rahmenwerten anhand der Entnahme zielstarker Bäume verdeutlichen. Das Element „Entnahme zielstarker Bäume“ ist zum Beispiel Teil der Strategie LÖWE. Ob während der Fortschreibung unter LÖWE tatsächlich eine Entnahme zielstarker Bäume stattfindet, hängt von dem artengruppenspezifischen Rahmenwert „Zielstärke“ und den tatsächlich erreichten Dimensionen der Einzelbäume im Bestand ab. Gibt es im betrachteten Bestand zum Zeitpunkt des Eingriffs keine zielstarken Bäume, so kann keine derartige Nutzung stattfinden.

Angenommen, es befinden sich zielstarke Bäume im Bestand, kann der Eingriff nur durchgeführt werden, wenn die geforderte Mindestmasse für Endnutzungen durch die Summe der Masse der zielstarken Bäume erreicht wird. Diese Mindestmasse wird durch den bestandestypspezifischen Rahmenwert "Minimales Endnutzungsvolumen“ gesetzt. In den folgenden Abschnitten wird erläutert, welche Rahmenwerte bei welchen Eingriffen (siehe Tabelle 3.5.c) eine Rolle spielen. 
Tabelle 3.5.c: Verwendete Maßnahmenelemente und ihre Zuordnung zu den Kategorien Schutz, Endnutzung, Durchforstung und Verjüngung.

\begin{tabular}{|c|c|c|c|}
\hline Kategorie & Maßnahmenelement & $\begin{array}{l}\text { Relevante } \\
\text { Rahmenwerte auf } \\
\text { Artenebene }\end{array}$ & $\begin{array}{l}\text { Relevante Rahmenwerte auf } \\
\text { Bestandesebene }\end{array}$ \\
\hline \multirow[t]{2}{*}{ Schutz } & Minderheitenschutz & & $\begin{array}{l}\text { Eingriffsturnus, } \\
\text { Schutz von Minderheiten }\end{array}$ \\
\hline & Habitatbaumauswahl & & $\begin{array}{l}\text { Eingriffsturnus, } \\
\text { Anzahl Habitatbäume }\end{array}$ \\
\hline \multirow[t]{6}{*}{ Endnutzung } & Prüfe Endnutzungsmasse & & $\begin{array}{l}\text { Eingriffsturnus, } \\
\text { minimales Endnutzungsvolumen }\end{array}$ \\
\hline & Zielstärkennutzung & Zielstärke & $\begin{array}{l}\text { Eingriffsturnus, } \\
\text { maximales Endnutzungsvolumen }\end{array}$ \\
\hline & Zielstärkennutzung mit Samenbäumen & $\begin{array}{l}\text { Zielstärke, } \\
\text { Ziel-Artenanteil }\end{array}$ & $\begin{array}{l}\text { Eingriffsturnus, } \\
\text { maximales Endnutzungsvolumen }\end{array}$ \\
\hline & $\begin{array}{l}\text { Zielstärkennutzung mit bedingten } \\
\text { Samenbäumen }\end{array}$ & $\begin{array}{l}\text { Zielstärke, } \\
\text { Ziel-Artenanteil }\end{array}$ & $\begin{array}{l}\text { Eingriffsturnus, } \\
\text { maximales Endnutzungsvolumen }\end{array}$ \\
\hline & Entnahme konkurrierender Z-Bäume & Zielstärke & $\begin{array}{l}\text { Eingriffsturnus, } \\
\text { maximales Endnutzungsvolumen }\end{array}$ \\
\hline & Entnahme einer erntereifen Schicht & Ziel-Dg & $\begin{array}{l}\text { Eingriffsturnus, } \\
\text { maximales Endnutzungsvolumen, } \\
\text { Endnutzungszeitraum }\end{array}$ \\
\hline \multirow[t]{10}{*}{ Durchforstung } & Prüfe Durchforstungsmasse & & $\begin{array}{l}\text { Eingriffsturnus, } \\
\text { minimales Durchforstungsvolumen }\end{array}$ \\
\hline & Prüfe Eingriffssmasse & & $\begin{array}{l}\text { Eingriffsturnus, } \\
\text { minimales Eingriffsvolumen }\end{array}$ \\
\hline & $\begin{array}{l}\text { permanente Z-Baum-Auswahl nach } \\
\text { Zieltyp }\end{array}$ & $\begin{array}{l}\text { Mindesthöhe für } \\
\text { den ersten Eingriff }\end{array}$ & $\begin{array}{l}\text { Eingriffsturnus, } \\
\text { angestrebter Zieltyp }\end{array}$ \\
\hline & $\begin{array}{l}\text { permanente Z-Baum-Auswahl nach } \\
\text { aktuellem Mischungsverhältnis }\end{array}$ & $\begin{array}{l}\text { Mindesthöhe für } \\
\text { den ersten Eingriff }\end{array}$ & Eingriffsturnus \\
\hline & $\begin{array}{l}\text { temporäre Z-Baum-Auswahl nach } \\
\text { Zieltyp }\end{array}$ & $\begin{array}{l}\text { Mindesthöhe für } \\
\text { den ersten Eingriff }\end{array}$ & $\begin{array}{l}\text { Eingriffsturnus, } \\
\text { angestrebter Zieltyp }\end{array}$ \\
\hline & $\begin{array}{l}\text { temporäre Z-Baum-Auswahl nach } \\
\text { aktuellem Mischungsverhältnis }\end{array}$ & $\begin{array}{l}\text { Mindesthöhe für } \\
\text { den ersten Eingriff }\end{array}$ & Eingriffsturnus \\
\hline & Freistellung der permanenten Z-Bäume & Freistellungsgrad & $\begin{array}{l}\text { Eingriffsturnus, } \\
\text { maximales Durchforstungsvolumen, } \\
\text { maximales Eingriffsvolumen }\end{array}$ \\
\hline & $\begin{array}{l}\text { Freistellung der permanenten Z-Bäume } \\
\text { von im Zieltyp nicht vorgesehenen Arten }\end{array}$ & $\begin{array}{l}\text { Freistellungsgrad, } \\
\text { Ziel-Artenanteil }\end{array}$ & $\begin{array}{l}\text { Eingriffsturnus, } \\
\text { maximales Durchforstungsvolumen, } \\
\text { maximales Eingriffsvolumen, } \\
\text { angestrebter Zieltyp }\end{array}$ \\
\hline & Freistellung der temporären Z-Bäume & Freistellungsgrad & $\begin{array}{l}\text { Eingriffsturnus, } \\
\text { maximales Durchforstungsvolumen, } \\
\text { maximales Eingriffsvolumen }\end{array}$ \\
\hline & $\begin{array}{l}\text { Freistellung der temporären Z-Bäume } \\
\text { von im Zieltyp nicht vorgesehenen Arten }\end{array}$ & $\begin{array}{l}\text { Freistellungsgrad, } \\
\text { Ziel-Artenanteil }\end{array}$ & $\begin{array}{l}\text { Eingriffsturnus, } \\
\text { maximales Durchforstungsvolumen, } \\
\text { maximales Eingriffsvolumen, } \\
\text { angestrebter Zieltyp }\end{array}$ \\
\hline Verjüngung & Pflanzung & Ziel-Artenanteil & angestrebter Zieltyp \\
\hline
\end{tabular}




\section{Schutz}

Werden Schutzelemente eingesetzt, können sie den Ablauf der Endnutzung und der Durchforstung beeinflussen.

Minderheitenschutz: Indem Bäume, die einer Minderheitenart im Bestand angehören, permanent markiert werden, bleiben sie bis zum Erreichen ihrer Zielstärke im Bestand erhalten und ihr Wachstum wird durch Freistellung des Baums gefördert (Maßnahmenelement „Minderheitenschutz"). Das unter „Durchforstung“ beschriebene Vorgehen der permanenten Markierung von Z-Bäumen ermöglicht solch eine Förderung von seltenen Arten. Ob dieser Minderheitenschutz eingesetzt wird, hängt von dem Rahmenwert „Schutz von Minderheiten“ ab.

Habitatbäume: Das Programm bietet als zweites mögliches Schutzelement die Möglichkeit, eine gewisse Anzahl von Habitatbäumen pro Hektar zu markieren und somit der Nutzung zu entziehen (Maßnahmenelement „Habitatbaumauswahl“). Die Anzahl der zu markierenden Bäume pro Hektar kann als Rahmenwert „Anzahl Habitatbäume“ gesetzt werden.

\section{Endnutzung}

Im wesentlichen lassen sich zwei Typen von Endnutzungselementen unterscheiden: Einerseits können Bäume einzeln entnommen werden, andererseits können ganze Bestandesschichten als Nutzungseinheit abgetrieben werden. Relevanter Rahmenwert auf Artenebene ist die „Zielstärke bzw. Ziel-Dg“. Diese wird bei Zielstärkennutzung als Zielstärke des Einzelbaums, bei schichtenweiser Nutzung als Ziel-Dg der Schicht begriffen. Auf Bestandesebene muß eine gewisse Mindestmasse bei Endnutzungen erreicht werden (Rahmenwert „Minimales Endnutzungsvolumen“), ansonsten wird kein Eingriff durchgeführt. Das Maßnahmenelement „Prüfe Endnutzungsmasse“ kontrolliert die Einhaltung der Mindestmasse. Auch ein maximales Endnutzungsvolumen (Rahmenwert „Maximales Endnutzungsvolumen“) darf nicht überschritten werden. Der Wert wird innerhalb der Endnutzungsroutinen (zum Beispiel im Maßnahmenelement „Zielstärkennutzung“) überprüft. Wird das maximale Volumen erreicht, bricht die jeweilige Endnutzungsrountine ab. Beim Einsatz schichtenweiser Nutzung steuert der Rahmenwert „Endnutzungszeitraum“, in welchem Zeitraum die Nutzung der Schicht abzuschließen ist.

Zielstärkennutzung: Beim Einsatz des Maßnahmenelements „Zielstärkennutzung“ werden Bäume beim Erreichen ihrer artenspezifischen Zielstärke entnommen. Dabei wird jeweils derjenige Baum zuerst entnommen, der seine Zielstärke am weitesten überschritten hat. Zusätzlich zu der Grundform stehen noch Endnutzungselemente zur Verfügung, welche die Zielstärkennutzung einstellen, wenn durch die Entnahme der Bäume die Erhaltung einer im Zieltyp geforderten Art gefährdet ist. Ziel dieser Elemente ist es, die Verjüngung der Art durch Erhaltung von Samenbäumen sicherzustellen. Eines dieser Elemente erhält die Samenbäume solange im Bestand, bis die nächste Generation dieser Art gesichert ist (Maßnahmenelement „Zielstärkennutzung mit Samenbäumen“). Ein zweites Element gibt die Möglichkeit, solche Samenbäume zu entnehmen, wenn sie ihren 
Zieldurchmesser um 20\% überschritten haben, um eine weitere Entwertung dieser Bäume zu verhindern (Maßnahmenelement „Zielstärkennutzung mit bedingten Samenbäumen“).

In jedem Fall kann es bei Überschreitung der Zielstärke zu unerwünschter Konkurrenz unter den zielstarken Bäumen kommen. Um das zu verhindern, kann ein Endnutzungselement eingesetzt werden, das zielstarke Bäume entnimmt, wenn sie mit anderen zielstarken Bäumen konkurrieren (Maßnahmenelement „Entnahme konkurrierender Z-Bäume“).

Nutzung einer Bestandesschicht: Die Nutzung der führenden Bestandesschicht beginnt, wenn der Dg der Schicht Erntereife (das heißt den gesetzten Zieldurchmesser) erreicht hat (Maßnahmenelement „Entnahme einer erntereifen Schicht“). Welcher Zieldurchmesser angestrebt wird, hängt von der dominierenden Baumart der zu erntenden Schicht ab. Die Entnahme beginnt analog zum Vorgehen bei der Zielstärkennutzung bei dem Baum, dessen BHD den Ziel-Dg der Schicht am weitesten überschritten hat und endet, wenn die maximale Endnutzungsmasse erreicht ist oder die erntereife Schicht vollständig entnommen wurde. Verbleiben nach dem ersten Eingriff noch Bäume der betrachteten Schicht im Bestand, so wird darauf hingearbeitet, daß alle verbleibenden Bäume der Schicht innerhalb eines Endnutzungszeitraums entnommen werden. Die Endnutzungszeiträume orientieren sich an artenspezifischen Verjüngungszeiträumen. Um die vollständige Ernte einer Schicht in einem gegebenen Zeitraum entnehmen zu können, sind angemessen hohe Endnutzungsvolumina (Rahmenwerte „maximales und minimales Endnutzungsvolumen“) erforderlich.

\section{Durchforstung}

Um eine Durchforstungsmaßnahme programmiertechnisch umzusetzen, werden zunächst freizustellende Bäume ausgewählt und permanent oder temporär markiert. In einem zweiten Schritt werden die markierten Bäume dann von Bedrängern freigestellt. Bei der Entnahme von Bäumen wird darauf geachtet, daß die minimalen Durchforstungsvolumina (Rahmenwert „minimales Durchforstungsvolumen“) nicht unter- und die maximalen Durchforstungsvolumina (Rahmenwert „maximales Durchforstungsvolumen“) nicht überschritten werden. Weiterhin wird das maximale und minimale Eingriffsvolumen berücksichtigt, das sich aus der Summe der in der Endnutzung angefallenen Masse und der in der Durchforstung angefallenen Masse zusammensetzt. Analog zur Volumenkontrolle der Endnutzung wird das Erreichen des minimalen Durchforstungsvolumens und des minimalen Eingriffsvolumens durch die Maßnahmenelemente „Prüfe Durchforstungsmasse“ und „Prüfe Eingriffsmasse“ gesteuert. Die Einhaltung der maximalen Massen wird direkt bei der Durchführung der Entnahme geprüft und gilt als Abbruchkriterium für eine Durchforstung.

Auswahl: Bei der Markierung der freizustellenden Bäume wird auf die Einhaltung einer Mindesthöhe geachtet. Somit wird die Höhe des ersten Eingriffs festgelegt (Rahmenwert Mindesthöhe für ersten Eingriff), da die Markierung von Bäumen eine Freistellung dieser Bäume nach sich zieht. 
Durch die permanente Markierung von Bäumen kann die kontinuierliche Pflege dieser Bäume bis zur Endnutzung sichergestellt und auch die Baumartenzusammensetzung langfristig gesteuert werden. Die Anzahl von permanent markierten Bäumen ist von ihrer erwarteten Kronengröße bei Erreichung des Zieldurchmessers abhängig. Bei Erreichung des Zieldurchmessers soll der Prozentsatz der Kronenschirmfläche einer Art in bezug zur Bestandesfläche dem Mischungsanteil entsprechen, welcher der einzelnen Art zugeordnet ist.

Neben der Auswahl permanenter Z-Bäume kann auch eine temporäre Markierung verwendet werden, die nur für eine Durchforstung Gültigkeit besitzt. Diese kann einerseits dazu genutzt werden, eine flächige Bestandespflege zwischen den permanent markierten Individuen zu gewährleisten. Andererseits kann durch die temporäre Markierung eine Bestandespflege realisiert werden, die nicht eine bestimmte Artenzusammensetzung anstrebt. Die Anzahl der temporär markierten Bäume kann je nach Pflegekonzept variieren.

Bei der Auswahl der freizustellenden Bäume wird eine gleichmäßige Verteilung der markierten Bäume auf der Fläche angestrebt. Sowohl für die permanente als auch für die temporäre Markierung werden zwei Arten von Auswahlalgorithmen zur Verfügung gestellt. Einerseits können bei der Auswahl nur Baumarten ausgewählt werden, die im Zieltyp erwünscht sind (Maßnahmenelemente „permanente Z-Baum-Auswahl nach Zieltyp“ und „temporäre Z-Baum-Auswahl nach Zieltyp“). Andererseits kann die Anzahl der auszuwählenden Bäume einer Art dem aktuellen Artenverhältnis entsprechend gesteuert werden (Maßnahmenelemente „permanente Z-Baum-Auswahl nach aktuellem Mischungsverhältnis“ und „temporäre Z-Baum-Auswahl nach aktuellem Mischungsverhältnis“). Durch die erste Möglichkeit kann die Artenzusammensetzung des Bestandes umgesteuert werden, wenn die im Zielbestand vorgesehenen Arten noch nicht im angestrebten Mischungsverhältnis zueinander stehen. Durch die zweite Möglichkeit wird eine Pflege des Bestandes auch dann ermöglicht, wenn der Bestand nur aus Bäumen besteht, die nicht im Zieltyp vorgesehen sind.

Entnahme von Bedrängern: In der eigentlichen Durchforstung werden die markierten „Zukunftsbäume“ (Z-Bäume) freigestellt. Der Freistellungsgrad eines zu pflegenden Baums ist definiert durch die Größe des freien Raumes, der seine Krone nach dem Eingriff umgeben soll. Je nach Baumart kann auch eine gewisse Überlappung der Kronen zugelassen werden. Der Rahmenwert „Freistellungsgrad“ gibt vor, ob eine Überlappung zugelassen ist (der Freistellungsgrad ist kleiner als 0) oder ein freier Raum um die Krone geschaffen wird (der Freistellungsgrad ist größer als 0). Befinden sich im freizustellenden Radius des Bezugsbaums potentielle Bedränger, werden die bedrängenden Bäume in der Reihenfolge entfernt, die ihrem Bedrängungspotential entspricht. Der Grad der Bedrängung mißt sich an den A-Werten der Nachbarn zu diesem Bezugsbaum. Hat der Bezugsbaum $90 \%$ seiner Zielstärke erreicht, wird er nicht mehr weiter freigestellt, da davon auszugehen ist, daß er das zusätzliche Raumangebot innerhalb des Zeitraums bis zu seiner Entnahme nicht mehr ausschöpfen kann und daß die ihn umgebenden Bäume die bei seiner Entnahme entstehende Lücke einnehmen sollen. Zusätzlich zu den Grundelementen „Freistellung der permanenten Z-Bäume“ und „Freistellung der temporären Z-Bäume“ kann die Entnahme von Bedrängern auch auf Arten beschränkt 
werden, die in der durch den Zieltyp vorgegebenen Zielbestockung nicht erwünscht sind (Maßnahmenelemente „Freistellung der permanenten Z-Bäume von im Zieltyp nicht vorgesehenen Arten“ und „Freistellung der temporären Z-Bäume von im Zieltyp nicht vorgesehenen Arten“). Diese Einschränkung stellt eine weitere Steuerungsmöglichkeit für die Baumartenzusammensetzung neben der artengesteuerten Auswahl der Z-Bäume dar.

\section{Verjüngung}

Da sich die eigentliche Wachstums- und Eingriffssimulation auf Bäume bezieht, die einen Mindest- Brusthöhendurchmesser (BHD) von $7 \mathrm{~cm}$ aufweisen, ist es für die weitere Bestandesentwicklung von Belang, wann, an welchen Stellen, mit welcher Art und in welcher Anzahl der Einwuchs neuer Individuen in diese Stärkeklasse stattfindet.

Einwuchs entstammt programmtechnisch aus drei Quellen in den Beständen: Erstens kann Einwuchs aus Verjüngung hervorgehen, die schon zum Ausgangszeitpunkt etabliert war (diese Bäume werden im System als Bäume „Vorhanden“ geführt). Zweitens kann Verjüngung einwachsen, die während des Simulationszeitraums gepflanzt wurde (diese Bäume gelten als "gepflanzt“). Drittens kann sich Einwuchs aus Naturverjüngung entwickeln, die nicht im Datenmaterial des Ausgangsbestandes auftaucht (solche Bäume werden als „naturverjüngt“ markiert).

Wenn das Ziel der Baumartenzusammensetzung durch die aktuelle Bestockung erreicht werden kann und keine Verjüngung existiert, die durch die Verjüngungssituation zum Zeitpunkt Null vorbestimmt ist, werden keine Pflanzungsmaßnahmen eingeleitet, und der Einwuchs besteht zu 100\% aus Naturverjüngung. Anderenfalls setzt sich der Einwuchs eines Bestandes zu einem Teil aus Individuen mit der Kennung „vorhanden“ oder "gepflanzt“ zusammen. Der Anteil richtet sich nach dem Flächenanteil, den die Verjüngung aus simulierter Pflanzung und vorbestimmter Verjüngung einnimmt.

Pflanzung: Eine Pflanzung (Maßnahmenelement „Pflanzung“) wird dann durchgeführt, wenn die angestrebten Haupt- und Nebenbaumarten weder im Bestand, noch in der Verjüngung ausreichend vertreten sind. Die Summe aus Kronenschirmflächenprozent im Bestand und Flächenprozent der Verjüngung muß mindestens $70 \%$ des Zielprozents (Rahmenwert „Ziel-Artenanteil“) erreichen, damit die Baumart als ausreichend vertreten angesehen wird. Pflanzungen werden zeitnah zu Endnutzungen durchgeführt. Um zu überprüfen, ob eine Endnutzung ansteht, wird überprüft, welcher Anteil der Bäume der führenden Bestandesschicht $80 \%$ ihres Zieldurchmessers (Rahmenwert „Zieldurchmesser") erreicht haben.

\subsubsection{Szenario LÖWE}

Die Komponenten für die Variante „LÖWE“ sind so ausgewählt und konfiguriert, daß sie die waldbaulichen Strategien in den Niedersächsischen Landesforsten wiedergeben.

Konkrete Handlungsanweisungen sind in den Merkblättern der Niedersächsischen Landesforstverwaltung (NIEDERSÄCHSISCHE LANDESFORSTVERWALTUNG 2006) oder der Richtlinie zur Baumartenwahl (Niedersächsische LandesforstVerwaltung 2004) enthalten. Gemäß 
diesen Bestimmungen wurden die Bestandesentwicklungs-, Endnutzungs-, Durchforstungs- und Verjüngungselemente entwickelt. Die in den Ablauf integrierten Zielvorgaben (aufgeführt unter "LÖWE" in den Tabellen 3.5.a, 3.5.b) orientieren sich ebenfalls an diesen Literaturquellen. Eingriffe in den Bestand sind in dieser Variante alle fünf Jahre vorgesehen. Die Maßnahmenkette sieht die folgenden Maßnahmenelemente vor und prüft ihre Anwendung gemäß der hier vorgestellten Reihenfolge:

Schutz:

- Habitatbaumauswahl

- Minderheitenschutz

Endnutzung:

- Zielstärkennutzung mit bedingten Samenbäumen

- Prüfe Endnutzungsmasse

Durchforstung:

- $\quad$ permanente Z-Baum-Auswahl nach Zieltyp

- $\quad$ Freistellung der permanenten Z-Bäume

- temporäre Z-Baum-Auswahl nach Zieltyp

- Freistellung der temporären Z-Bäume

- temporäre Z-Baum-Auswahl nach aktuellem Mischungsverhältnis

- Freistellung der temporären Z-Bäume von im Zieltyp nicht vorgesehenen Arten

- Freistellung der temporären Z-Bäume

- Prüfe Durchforstungsmasse

- Prüfe Entnahmemasse

Verjüngung:

- Pflanzung

\section{Schutz}

Das LÖWE-Programm sieht in seinem 7. Grundsatz die „Erhaltung alter Bäume und den Schutz seltener und bedrohter Pflanzen- und Tierarten“ vor (NIEDERSÄCHSISCHE LANDESFORSTVERWALTUNG 1991). Um diesem Grundsatz zu entsprechen, wird vor dem ersten simulierten Eingriff jeweils ein Baum pro Hektar als Habitatbaum markiert. Weiterhin wird durch die Z-Baum-Markierung jeweils eines Baums jeder auf der Bestandesfläche vorkommenden Art, dem Schutz und der Erhaltung seltener Baumarten sowie einer größtmöglichen Artenmischung Rechnung getragen. 


\section{Endnutzung}

Der 6. Grundsatz des LÖWE-Programms fordert, daß Wald wenn möglich einzelstammoder gruppenweise bei Hiebsreife genutzt werden soll. Dieser Forderung wird durch den Einsatz von Zielstärkennutzung Rechnung getragen. Da vorwiegend mit natürlicher Verjüngung gearbeitet werden soll (Grundsatz IV), wird das Maßnahmenelement „Zielstärkennutzung mit bedingten Samenbäumen“ eingesetzt.

\section{Durchforstung}

Mit der Durchforstung sollen ausgewählte Z-Bäume gepflegt und gefördert werden; wenn nötig, soll auch eine Umsteuerung der Zusammensetzung des Bestandes ermöglicht werden. Es werden zunächst die permanent zu pflegenden Z-Bäume ausgewählt. Die Anzahl der Z-Bäume einer Art orientiert sich daran, welchen Anteil die Art im Zielbestand einnehmen soll (Maßnahmenelement „permanente Z-Baum-Auswahl nach Zieltyp“). Diese Z-Bäume werden anschließend freigestellt (Maßnahmenelement „Freistellung der permanenten Z-Bäume“). Um eine Pflege auf ganzer Fläche zu gewährleisten, werden einige Individuen zwischen den permanent markierten Bäumen temporär ausgewählt und freigestellt. Es werden zunächst nur Markierungen an Bäumen vorgenommen, die einer nach dem angestrebten WET erwünschten Art angehören (Maßnahmenelement „temporäre Z-Baum-Auswahl nach Zieltyp“). Im Vergleich zu den permanent markierten Bäumen werden hier allerdings erheblich mehr Individuen ausgewählt. Ihre Anzahl berechnet sich aus dem Quotienten der Bestandesfläche zur aktuellen Kronenschirmfläche, die ein Baum dieser Art mit dem durchschnittlichen Durchmesser zur Zeit der Betrachtung einnimmt.

Können nach diesen Kriterien nicht genügend Bäume markiert werden, wird das übrige Kontingent der temporär zu markierenden Bäume den aktuell vorhandenen Baumartenprozenten entsprechend ausgeschöpft. Bei der Entnahme werden zunächst diejenigen Bedränger entnommen, die zu Arten gehören, die nicht explizit in der Beschreibung des WET aufgeführt sind. Dadurch werden in den Räumen zwischen den ZBäumen die WET-konformen Arten besonders gefördert.

Überschreitet das Volumen der Entnahme die vorgesehenen Mindestmassen, werden die Durchforstungs- und Endnutzungsmaßnahmen tatsächlich durchgeführt. Andernfalls werden die Markierungen der zu entnehmenden Bäume wieder entfernt (Maßnahmenelemente „Prüfe Durchforstungsmasse“ und „Prüfe Entnahmemasse“).

\section{Verjüngung}

Da Naturverjüngung erwünscht ist, wird sie eingesetzt, wenn der Umbau aus der gegebenen Situation heraus durchführbar ist oder kein Umbaubedarf besteht. Um einen bestimmten WET erreichen zu können, werden Pflanzungen nur dann vorgenommen, wenn die aktuelle Artenzusammensetzung im Bestand nicht in eine dem WET entsprechende Mischung überführt werden kann. 


\subsubsection{Szenario PNV}

Mit der Variante PNV wird die Entwicklung der Baumartenzusammensetzung stark in Richtung der potentiell natürlichen Vegetation ( $p n V)$ gesteuert. Es werden dazu die Baumarten stark gefördert die der pnV entsprechen (nach KAISER UND ZACHARIAS 2003). Wie LÖWE vollzieht auch das Szenario „PNV“ alle fünf Jahre forstliche Eingriffe in den Beständen. Die Rahmenwerte orientieren sich an den Rahmenwerten von LÖWE. Lediglich der Freistellungsgrad der Bäume ist höher, um den Umbau in Richtung pnV zu beschleunigen.

Die Maßnahmenkette sieht folgende Schritte vor:

Schutz:

- $\quad$ keiner

Endnutzung:

- Zielstärkennutzung mit Samenbäumen

- Entnahme konkurrierender Z-Bäume

- Prüfe Endnutzungsmasse

Durchforstung:

- $\quad$ permanente Z-Baum-Auswahl nach Zieltyp

- Freistellung der permanenten Z-Bäume von im Zieltyp nicht vorgesehenen Arten

- Freistellung der permanenten Z-Bäume

- temporäre Z-Baum-Auswahl nach Zieltyp

- Freistellung der temporären Z-Bäume von im Zieltyp nicht vorgesehenen Arten

- $\quad$ Freistellung der temporären Z-Bäume

- temporäre Z-Baum-Auswahl nach aktuellem Mischungsverhältnis

- $\quad$ Freistellung der temporären Z-Bäume von im Zieltyp nicht vorgesehenen Arten

- Prüfe Durchforstungsmasse

- Prüfe Entnahmemasse

Verjüngung:

- Pflanzung

\section{Schutz}

Die Variante PNV kennt keine Schutzelemente. Oberstes Ziel ist die naturnahe Artenzusammensetzung. 


\section{Endnutzung}

Mit der Zielstärkennutzung fördert man die in der pnV des Untersuchungsgebiets dominierende Schattbaumart Buche. Im Gegensatz zum Szenario LÖWE wird jedoch auf das Element „Zielstärkennutzung mit Samenbäumen“ zurückgegriffen. Ist die Verjüngung einer Art, die für das Erreichen des PNV-Typs notwendig ist, noch nicht gesichert, werden Samenbäume im Bestand erhalten, bis sich die Verjüngungssituation geändert hat. So bleiben hiebsreife Bäume als Samenbäume länger erhalten als in der Variante LÖWE. Dabei wird ein Ertragsverlust durch die Überschreitung der angestrebten Zielstärke in Kauf genommen. Da starke Konkurrenzsituationen zwischen Samenbäumen, die den Zieldurchmesser weit überschritten haben, eintreten können, wird zusätzlich das Element „Entnahme konkurrierender Z-Bäume“ eingesetzt.

\section{Durchforstung}

Mit Durchforstungsmaßnahmen kann ein erheblicher Einfluß auf die künftige Artenzusammensetzung im Bestand genommen werden. Um dieses Potential voll auszuschöpfen, unterscheiden sich die im PNV-Konzept verwendeten Maßnahmen insofern von denen des LÖWE-Konzepts, als daß auch bei der Freistellung permanent markierter Bäume bevorzugt Bedränger entnommen werden, die nicht den erwünschten Arten angehören. Der Freistellungsgrad der Z-Bäume ist höher als in der LÖWE-Variante, so daß eine starke Begünstigung pnV- konformer und somit im Endbestand erwünschter Baumarten erzielt wird.

\section{Verjüngung}

Kongruent zur Variante "LÖWE“ werden Pflanzungen dann vorgenommen, wenn die aktuelle Artenzusammensetzung eines Bestandes nicht in eine dem PNV-Typ entsprechende Mischung überführt werden kann. Ist der Umbau aus der gegebenen Situation heraus durchführbar, wird mit Einwuchs aus Naturverjüngung gearbeitet.

\subsubsection{Szenario ERTRAG}

Um eine ertragsorientierte Waldbehandlung umzusetzen, wird in dieser Variante unter anderem ein Umbau der Istbestockungen in ertragsstarke Baumarten forciert. Desweiteren werden die Holzerntekosten durch stärkere Durchforstungen und längere Durchforstungsintervalle (alle zehn Jahre) sowie höhere Endnutzungsmassen gesenkt. Letztere können durch baumartenabhängige Endnutzungszeiträume, an deren Ende die Nutzung der führenden Bestandesschichten steht, und angepaßte Endnutzungsvolumina erreicht werden. Im Vergleich zu den beiden bereits vorgestellten Varianten verkürzen sich die Umtriebszeiten auf lange Sicht, weil die Bäume mit Erreichen eines geringeren BHDs endgenutzt werden. Die Wälder entwickeln sich bei dieser Variante zu gleichaltrigen Reinbeständen, in denen Minderheiten oder Habitatbäume nicht geschützt werden. 
Die Maßnahmenkette stellt sich wie folgt dar:

Schutz:

- $\quad$ keiner

Endnutzung:

- Entnahme einer erntereifen Schicht

- Prüfe Endnutzungsmasse

Durchforstung:

- $\quad$ permanente Z-Baum-Auswahl nach Zieltyp

- $\quad$ Freistellung der permanenten Z-Bäume von im Zieltyp nicht vorgesehenen Arten

- Freistellung der permanenten Z-Bäume

- $\quad$ temporäre Z-Baum-Auswahl nach Zieltyp

- $\quad$ Freistellung der temporären Z-Bäume von im Zieltyp nicht vorgesehenen Arten

- Freistellung der temporären Z-Bäume

- $\quad$ temporäre Z-Baum-Auswahl nach aktuellem Mischungsverhältnis

- Freistellung der temporären Z-Bäume von im Zieltyp nicht vorgesehenen Arten

- Prüfe Durchforstungsmasse

- Prüfe Entnahmemasse

Verjüngung:

- Pflanzung

\section{Endnutzung}

Damit hohe Endnutzungsmassen anfallen und gleichaltrige Reinbestände entstehen oder erhalten bleiben, werden die Bestände schichtenweise genutzt. (Maßnahmenelement „Entnahme einer erntereifen Schicht“). Im Vergleich zu den zuvor beschriebenen Varianten dürfen verhältnismäßig hohe Massen entnommen werden. Diese Vorgabe ermöglicht, daß die Holzernte effizienter durchgeführt werden kann, wodurch die Holzerntekosten sinken. Zum Ende der Endnutzungsperiode werden alle bis dahin verbliebenen Bäume der zu nutzenden Schichten, ungeachtet von Mindest- oder Maximalmengen entnommen.

\section{Durchforstung}

Die zu pflegenden Individuen werden analog zu der Auswahl nach der PNV-Strategie identifiziert. Wie bei dem Konzept „PNV“ werden die Z-Bäume bei der Ertragsvariante stark freigestellt. Vorrangig werden unter den Bedrängern der Z-Bäume die Bäume 
ertragsschwacher Baumarten entnommen. Es werden deutlich höhere Massen je Durchforstung entnommen als in den oben beschriebenen Szenarien, um den Einsatz von Harvestern zu fördern.

\section{Verjüngung}

Analog zu den beiden schon beschriebenen Varianten werden Pflanzungen vorgenommen, sofern die angestrebten Reinbestände nicht aus der gegebenen Bestandessituation heraus entwickelt werden können. Die Pflanzroutine wird aktiviert, wenn eine Pflanzung zu dem betrachteten Zeitpunkt waldbaulich sinnvoll ist. Die baumartenbezogenen Endnutzungszeiträume sind so gewählt, daß sie das Ankommen von Naturverjüngung begünstigen.

\subsubsection{Szenario PROZESS}

Bei der Variante „PROZESS“ sollen natürliche Prozesse im Wald genutzt und unterstützt werden, indem zum Beispiel konkurrenzstarke Bäume zusätzlich gefördert werden. Es wird darauf verzichtet, bestimmte Mischungsverhältnisse bei den beteiligten Baumarten anzustreben, um die natürliche Entwicklungsdynamik nicht zu stören. Die konkurrenzstärkeren Baumarten setzen sich im Bestand durch. Die Förderung von Bäumen durch die Entnahme ihrer Bedränger fällt im Vergleich zu den anderen Szenarien eher schwach aus (siehe Freistellungsgrad in Tabelle 3.5.b). Es wird mit wenigen Eingriffen alle zehn Jahre gearbeitet, um dem Bestand die Möglichkeit zu geben, sich zwischen diesen Behandlungen natürlich differenzieren können. Um in der Endnutzungsphase nur kleinflächige Störungen zu verursachen, werden zielstarke Bäume individuell genutzt. Dies reduziert die Verjüngungsmöglichkeiten für lichtbedürftige Baumarten. Pflanzungen gehören nicht zu diesem Konzept.

Schutz:

- Habitatbaumauswahl

Endnutzung:

- Zielstärkennutzung

- Prüfe Endnutzungsmasse

Durchforstung:

- temporäre Z-Baum-Auswahl nach aktuellem Mischungsverhältnis

- Freistellung der temporären Z-Bäume

- Prüfe Durchforstungsmasse

- Prüfe Entnahmemasse

Verjüngung:

- $\quad$ keine Pflanzung 


\section{Schutz}

Zum PROZESS-Konzept gehört die Auswahl von Habitatbäumen, da alle Alters- und Zerfallsstufen als Bestandteil natürlicher Prozesse begriffen werden. Da keine bestimmten Baumartenzusammensetzungen angestrebt werden, wird auf den Schutz von Minderheiten verzichtet.

\section{Endnutzung}

Es werden zielstarke Baumarten geerntet, da das Szenario zwar die natürlichen Prozesse unterstützt, auf eine Nutzung aber nicht verzichtet. Die Zielstärken orientieren sich an den Produktionszielen gemäß der „Richtlinie zur Baumartenwahl“ (NIEDERSÄCHSISCHE LANDESFORSTVERWALTUNG 2004), die auch in den Varianten „LÖWE“ und „PNV“ verwendet werden. In der Variante PNV wird aber nur alle zehn Jahre in die Beständen eingegriffen. Samenbäume werden nicht im Bestand belassen, um die Mischung der Baumarten nicht aktiv zu beeinflussen.

\section{Durchforstung}

Bei den Durchforstungsmaßnahmen wird auf eine permanente Markierung von Z-Bäumen verzichtet, weil keine bestimmten Bestandestypen angestrebt werden. Die zu den Durchforstungszeitpunkten jeweils stärksten Individuen sollen sich ungeachtet ihrer Art durchsetzen. Entsprechend ihrer sozialen Stellung bedürfen diese Bäume nur einer geringen Förderung, so daß die Durchforstungsmassen trotz des zehnjährigen Durchforstungsintervalls relativ gering sind.

\subsection{Auswertung}

Die Auswertungsmöglichkeiten für die Simulationsergebnisse sind vielfältig. Aus den vorliegenden Einzelbauminformationen können Daten vieler verschiedener Kollektive (zum Beispiel nach Art und Alter oder Höhe und Art getrennt) aggregiert und ausgegeben werden. Das Simulationsmodell ist in der Lage auch positionsabhängige Strukturparameter zu liefern. Für jeden Bestand, aber auch auf Landschafts- oder Betriebsebene werden Summen- und Durchschnittswerte generiert. Weiterhin wird die Möglichkeit genutzt, Ergebnisse mit GIS-Bezug zu erstellen. So können zum Beispiel die Landschaftsdiversität untersucht oder Ergebnisse in Karten optisch übersichtlich dargestellt werden.

Die Vielfalt der Auswertungsmöglichkeiten zwingt zu einer Einschränkung auf einen Satz von Auswertungsparametern. Die Auswertung jedes theoretisch möglichen Parameters würde zu einer unübersichtlichen Informationsflut führen, die keine Unterstützung bei der Entscheidungsfindung sein kann. Im folgenden werden die ausgewählten Parameter vorgestellt. Anhand dieser werden im Ergebnisteil (siehe Kapitel 4) die Auswirkungen der angewendeten forstlichen Managementstrategien beleuchtet. 


\subsubsection{Auswahl der Auswertungsparameter}

Der erste Teil der gewählten Auswertungsparameter (siehe Tabelle 3.6.2.a) lehnt sich an den Katalog der gesamteuropäischen Kriterien einer nachhaltigen Forstwirtschaft an, der bei der dritten Ministerkonferenz zum Schutz der Wälder in Europa 1998 in Lissabon von den europäischen Forstministerien unter dem Namen Resolution L2 verabschiedet wurde. In diesem Katalog werden sechs Kriterien der Nachhaltigkeit genannt:

I. Forstliche Ressourcen

II. Gesundheit und Vitalität

III. Produktionsfunktion

IV. Biologische Diversität

V. Schutzfunktionen

VI. Sozio-Ökonomische Funktionen

Anhand ausgewählter Parameter, die die Erfüllung der Kriterien meßbar werden lassen, kann die Zielerreichung der Strategien bezüglich gesetzter Nachhaltigkeitsziele quantitativ überprüft werden. Wegen der Fülle an möglichen Ausgabeparametern beschränkt sich diese Arbeit auf Parameter der drei Kriterien Forstliche Ressourcen (I), Produktionsfunktion (III) und Biologische Diversität (IV). Diesen drei Kriterien wird im Rahmen einer Nachhaltigkeitsanalyse eine höhere Bedeutung als den drei nicht untersuchten Kriterien (II, V und VI) zugemessen (siehe NFV UND NFA 2002).

Zu I: Als wichtigsten Bereich für die nachhaltige Nutzung forstlicher Ressourcen wird der Indikator "Landnutzung und Forstfläche“ gesehen (siehe NFV UND NFA 2002). Weitere Parameter sind der „Holzvorrat“ und die „Kohlenstoffbilanz“. Da eine Änderung der Landnutzung oder eine Zu- oder Abnahme der Forstfläche nicht Teil der Simulation ist, stützt sich die Auswertung in dieser Arbeit auf den Holzvorrat, der eng mit dem GesamtKohlenstoffvorrat verknüpft ist. Auf eine explizite Auswertung des Kohlenstoffvorrates wird verzichtet. Einerseits soll eine Informationsredundanz (Holzvorrat - Kohlenstoffvorrat) vermieden werden, andererseits ist der Holzvorrat für den Waldbesitzer von größerem Interesse als der Kohlenstoffvorrat.

Die nachhaltige Verfügbarkeit der Ressource Holz definiert sich über die vorhandene Gesamtmasse und ihre Aufteilung in verschiedene Holzarten und Dimensionen. Die durch das Programm erzeugten Einzelbauminformationen (Masse, potentielle Erlöse bei sofortiger Ernte, potentielle Kosten bei sofortiger Ernte) lassen sich zu beliebigen Gruppeninformationen verdichten. In dieser Arbeit werden die Parameter Derbholzvorrat je Hektar und der Wert des stehenden Holzes als Abtriebswert angegeben. Ergänzend wird die Durchmesserstruktur der Bestände genauer betrachtet.

Zu II: Auf eine Auswertung des Kriteriums „Gesundheit und Vitalität“ wird verzichtet. 
Zu III: Die Produktionsfunktion des Waldes kann durch die Handlungsfelder „Holzproduktion“, „Nichtholzproduktion“ und „ökonomische Aspekte" charakterisiert werden (siehe NFV und NFA 2002).

Die Produktion des nachwachsenden Rohstoffs Holz ist mit dem Zuwachs der Bestände eng verknüpft. Eine nachhaltige Nutzung ist nur dann möglich, wenn die Entnahmemengen den Zuwachs nicht dauerhaft übersteigen. Die wichtigsten Kenngrößen sind daher das Volumen des Zuwachses und der Nutzung und das Verhältnis der beiden Größen zueinander. Es werden daher folgende Werte berechnet: Zuwachs und Nutzungsmassen (getrennt nach Vor-/ Endnutzung) in bezug auf alle Baumarten und nach Baumarten getrennt. Aus diesen Werten lassen sich auch Nutzungsprozente herleiten.

Da Nichtholzprodukte nicht Gegenstand des verwendeten Waldwachstumssimulators sind, wird auf eine Abbildung dieses Handlungsfelds verzichtet.

Als geeignete Parameter des Handlungsfelds der ökonomischen Aspekte kommen zum Beispiel das Verhältnis von Aufwand zu Ertrag, das Betriebsergebnis oder die Preisentwicklung für Leitsortimente in Frage. Der Simulator bietet die Möglichkeit, Kosten und Erträge der durchgeführten Erntemaßnahmen im Simulationszeitraum zu bewerten. $\mathrm{Da}$ die zu erwartende Preisentwicklung von vielen Faktoren abhängig ist, die nicht Gegenstand der Simulation sind, wird von ihrer Prognose abgesehen. Den Bewertungen liegen vielmehr Durchschnittspreise und Durchschnittserlöse der letzten zehn Jahre zugrunde. Die Einzelbauminformationen werden in den Parametern erntekostenfreie Holzerlöse aus Vornutzungen- und erntekostenfreie Holzerlöse aus Endnutzungen für die Bestände zusammengefaßt. Als weitere Kostenfaktoren müssen neben dem Einsatz von Arbeit und Maschinen bei Erntemaßnahmen auch Material- und Arbeitsaufwand bei Pflanzungen berücksichtigt werden. Als Maß für die anfallenden Kosten in diesem Bereich wird die - nach Baumartengruppen getrennte - Pflanzfläche herangezogen. Über Pflanzungskostenfunktionen kann dann der finanzielle Aufwand für Pflanzungsmaßnahmen auf dieser Pflanzfläche abgeschätzt werden. Der Holzproduktionswert dient als Anhaltspunkt für den betriebswirtschaftlichen Gesamterfolg der Strategie.

Zu IV: Für die Messung biologischer Diversität werden als wichtigste Handlungsfelder „repräsentative, seltene und gefährdete Waldökosysteme“, „Mehrfachnutzung“ und „Landschaftsdiversität“ genannt (siehe NFV UND NFA 2002). Danach folgen „Gefährdete Arten“ und die „Biodiversität in Wirtschaftswäldern“.

Der Status repräsentativer, seltener und gefährdeter Waldökosysteme wird in der Simulation als gegeben angesehen. Der Schutzstatus der Waldflächen oder eine Änderung dieses Status findet keine Berücksichtigung in der Simulation. Die Auswirkungen verschiedener Managementstrategien werden lediglich bei uneingeschränkter wirtschaftlicher Nutzbarkeit untersucht. Auch die multifunktionale Nutzung der Flächen oder eine Änderung der Nutzung ist nicht Gegenstand der auf Holznutzung beschränkten Simulation. Aus diesen Gründen sind die Handlungsfelder „Repräsentative, seltene und gefährdete Waldökosysteme“ und „Mehrfachnutzung“ nicht Gegenstand der Auswertung. 
Um die Landschaftsdiversität zu beschreiben, wird die Anordnung der Flächen gleicher Bestandestypen untersucht. Dazu wird aus der vektorenbasierten Karte der Bestandestypenverteilung eine Rasterkarte erstellt. In einer auf Rasterzellen basierenden Auswertung wird untersucht, ob eine Rasterzelle von Rasterzellen desselben oder eines anderen Typen umgeben wird. So wird ermittelt, ob die Landschaft aus kleinflächigen Einheiten oder großen, mehr oder weniger gleichförmigen Komplexen besteht. Je häufiger sich Rasterzellen verschiedener Bestandestypengruppen im Untersuchungsgebiet abwechseln, desto höher steigt der berechnete Landschafts-Durchmischungskoeffizient.

Da die Simulation auf Baumarten beschränkt ist, läßt sich die Zunahme oder der Rückgang gefährdeter Tier- und Pflanzenarten nur indirekt, zum Beispiel über einen Zusammenhang zwischen Bestandestyp und Anzahl der gefährdeten Tier- und Pflanzenarten, ermitteln. Auf eine Auswertung dieses Indikators wird daher verzichtet.

Der Index-A nach Pretzsch (PRETzSCH 1996) wurde als ein Parameter gewählt, um die Diversität in Wirtschaftswäldern abzubilden. Er berücksichtigt als vertikales Artprofil sowohl die Anzahl der Baumarten als auch ihre Zugehörigkeit zu Höhenschichten. Durch diesen Index kann ausgedrückt werden, wie sich die Bewirtschaftungsmaßnahmen der Strategien auf die Strukturvielfalt der Bestände auswirken.

In Wirtschaftswäldern wird die natürliche Alterungs- und Zerfallsphase der Bäume durch die Nutzung bei vielen Bäumen verhindert. Durch Angabe des Totholzvolumens wird festgehalten, wieviel ungenutztes Totholz in den Beständen durch natürliche Mortalität entsteht. Habitatbäume tragen zur Erhaltung von Organismen bei, die an Alterungs- und Zerfallsphasen gebundene Lebensräume angepaßt sind. Beispiele für solche Organismen sind Baumhöhlenbewohner, Insekten, Pilze, Moose und Flechten. Das Habitatbaumvolumen sagt aus, wieviel Holz durch Unter-Schutz-Stellung aktiv der Nutzung entzogen wird.

Mißt man den Erfolg der Strategien einzig an Indikatoren der Nachhaltigkeit, so wird der Aspekt außer acht gelassen, daß die Landschaft sich während des Simulationszeitraums noch in einer Umbauphase befinden kann. Die in der betrachteten Periode erzielten Ergebnisse werden zwar von den in diesem Zeitraum durchgeführten Maßnahmen beeinflußt, sie hängen aber auch von der bis dato betriebenen Wirtschaftsweise ab. Je nachdem, ob die neue Strategie sich stark von der bislang umgesetzten unterscheidet, ist eine kürzere oder längere Umbauphase zu erwarten.

Es werden zusätzlich Parameter berechnet, die den Erfolg der einzelnen Strategien im Hinblick auf die Erreichung eines angestrebten Mischungsanteils charakterisieren. Das Zielerreichungsprozent sagt aus, wie weit die aktuelle Artenzusammensetzung der Bestände von der angestrebten Mischung entfernt ist. Ein Vergleich des Endzustands mit dem Ausgangszustand zeigt, ob sich die Bestände durch die simulationsabhängigen Eingriffe während des Simulationszeitraums dem angestrebten Mischungsanteil annähern oder sich sogar von ihm entfernen. Durch die Auswertung des Bestandestyps kann das Erreichen einer gewünschten Bestandestypenverteilung auf Landschaftsebene überprüft werden. 
Zu V und VI: Auf eine Auswertung der Kriterien „Schutzfunktion“ und „Sozio-Ökonomische Funktionen" wird verzichtet.

\subsubsection{Herleitung der Auswertungsparameter}

Alle Parameter, die auf den Gesamtbestand oder auf Artengruppen des Bestandes bezogen sind, können auf Landschaftsebene zu Durchschnittswerten für verschiedene Kollektive (Landschaftsteile, Summe mehrerer Baumartengruppen) aggregiert werden (siehe Tabelle 3.6.2.a). In der Regel werden die Auswertungsparameter auf der Landschaftsebene betrachtet, eine feinere Untergliederung der Ergebnisse kann zur Diskussion der Werte herangezogen werden.

Tabelle 3.6.2.a: Übersicht über die Auswertungsparameter der Simulation zu den Kriterien „,forstliche Ressourcen“, „Produktionsfunktion“, „Biologische Diversität" und „Zielerreichung“.

\begin{tabular}{|c|c|c|c|c|c|}
\hline Kriterium & Parameter & $\begin{array}{l}\text { Landschafts- } \\
\text { bezug }\end{array}$ & Bestandesbezug & $\begin{array}{l}\text { Artengruppen- } \\
\text { bezug }\end{array}$ & $\begin{array}{l}\text { Nur nach } \\
\text { Fortschreibung } \\
\text { möglich }\end{array}$ \\
\hline \multirow{3}{*}{$\begin{array}{l}\text { Forstliche } \\
\text { Ressourcen }\end{array}$} & Derbholzvorrat & ja & ja & ja & nein \\
\hline & Durchmesserstruktur & ja & ja & ja & nein \\
\hline & Abtriebswert & ja & ja & ja & nein \\
\hline \multirow{7}{*}{$\begin{array}{l}\text { Produktions- } \\
\text { funktion }\end{array}$} & Vornutzungsmasse & ja & ja & ja & ja \\
\hline & Endnutzungsmasse & ja & ja & ja & ja \\
\hline & Zuwachs & ja & ja & ja & ja \\
\hline & $\begin{array}{l}\text { erntekostenfreie } \\
\text { Holzerlöse } \\
\text { Vornutzungen }\end{array}$ & ja & ja & ja & ja \\
\hline & $\begin{array}{l}\text { erntekostenfreie } \\
\text { Holzerlöse } \\
\text { Endnutzungen }\end{array}$ & ja & ja & ja & ja \\
\hline & Pflanzfläche & ja & ja & ja & ja \\
\hline & Holzproduktionswert & ja & ja & ja & ja \\
\hline \multirow[t]{4}{*}{$\begin{array}{l}\text { Biologische } \\
\text { Diversität }\end{array}$} & $\begin{array}{l}\text { Durchmischungs- } \\
\text { koeffizient }\end{array}$ & ja & nein & nein & nein \\
\hline & Index- $A$ & ja & ja & nein & nein \\
\hline & Totholzvolumen & ja & ja & ja & ja \\
\hline & Habitatbaumvolumen & ja & ja & ja & ja \\
\hline \multirow[t]{2}{*}{ Zielerreichung } & Zielerreichungsprozent & ja & ja & nein & nein \\
\hline & $\begin{array}{l}\text { Verteilung der } \\
\text { Bestandestypen }\end{array}$ & ja & nein & nein & nein \\
\hline
\end{tabular}

\section{Derbholzvorrat}

Der Derbholzvorrat wird über die Summe der Derbholzmasse der Einzelbäume in Vorratsfestmetern pro Hektar berechnet. Es werden die Volumenfunktionen aus BWINPro verwendet (siehe NAGEL 2005). Sie richten sich nach Baumart, Höhe und Durchmesser des betrachteten Baums. 


\section{Durchmesserstruktur}

Die Durchmesserstruktur gibt die prozentuale Verteilung des Derbholzvolumens im Bestand über 17 Durchmesserklassen an. Die Durchmesserklassen sind in $5 \mathrm{~cm}$-Stufen gestaffelt. Die niedrigste Durchmesserstufe beginnt bei $5 \mathrm{~cm}$, die zweite bei $10 \mathrm{~cm}$ und so weiter. Eine Durchmesserklasse von 0 bis kleiner $5 \mathrm{~cm}$ wird nicht benötigt, da Bäume mit einem BHD von unter $5 \mathrm{~cm}$ nicht Gegenstand der Simulation sind. Die 17. Klasse faßt alle Bäume mit einem BHD von $85 \mathrm{~cm}$ und mehr zusammen.

\section{Abtriebswert}

Der aktuelle Wert Bestände wird durch die Angabe des erntekostenfreien Abtriebswerts ausgedrückt. Er berechnet sich aus dem Verkaufserlös des stehenden Holzes, der um die anfallenden Erntekosten reduziert wird. Jedem Baum werden ein potentieller Erlöswert und potentielle Erntekosten zugeordnet, die bei einer Nutzung zum betrachteten Zeitpunkt anfallen würden.

Die hinterlegten Funktionen ${ }^{13}$ richten sich nach Baumart und BHD des jeweiligen Baums. Der bessere Pflegezustand permanenter Z-Bäume wirkt sich auf die Qualität dieser Bäume positiv aus. Ihnen wird eine höhere Wertklasse unterstellt und sie werden daher mit einer anderen Funktion bewertet (siehe Abbildung 3.6.2.a) als andere Bäume (siehe Abbildung 3.6.2.b).

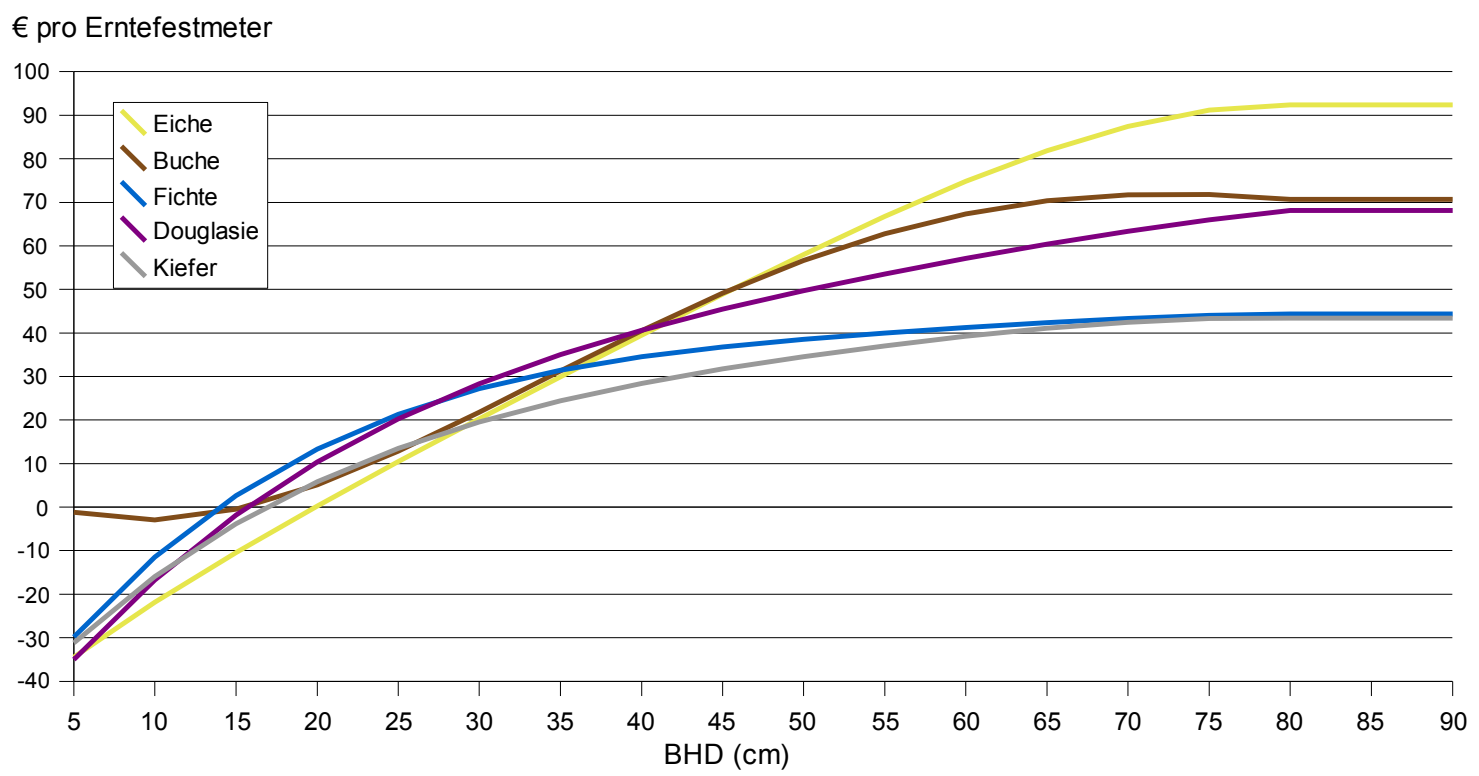

Abbildung 3.6.2.a: Erntekostenfreie Holzerlöse ${ }^{13}$ für Bäume der Wertklasse II.

13 Die unveröffentlichten Funktionen wurden vom Institut für Forstökonomie der Universität Göttingen unter der Leitung von Prof. Dr. B. Möhring im Rahmen des BMBF-Projekts „Biosphärenforschung Integrative und anwendungsorientierte Modellprojekte“ (kurz: BioTeam), Teilprojekt „FOREST“ (Fkz. 01 LM 0207) entwickelt. 


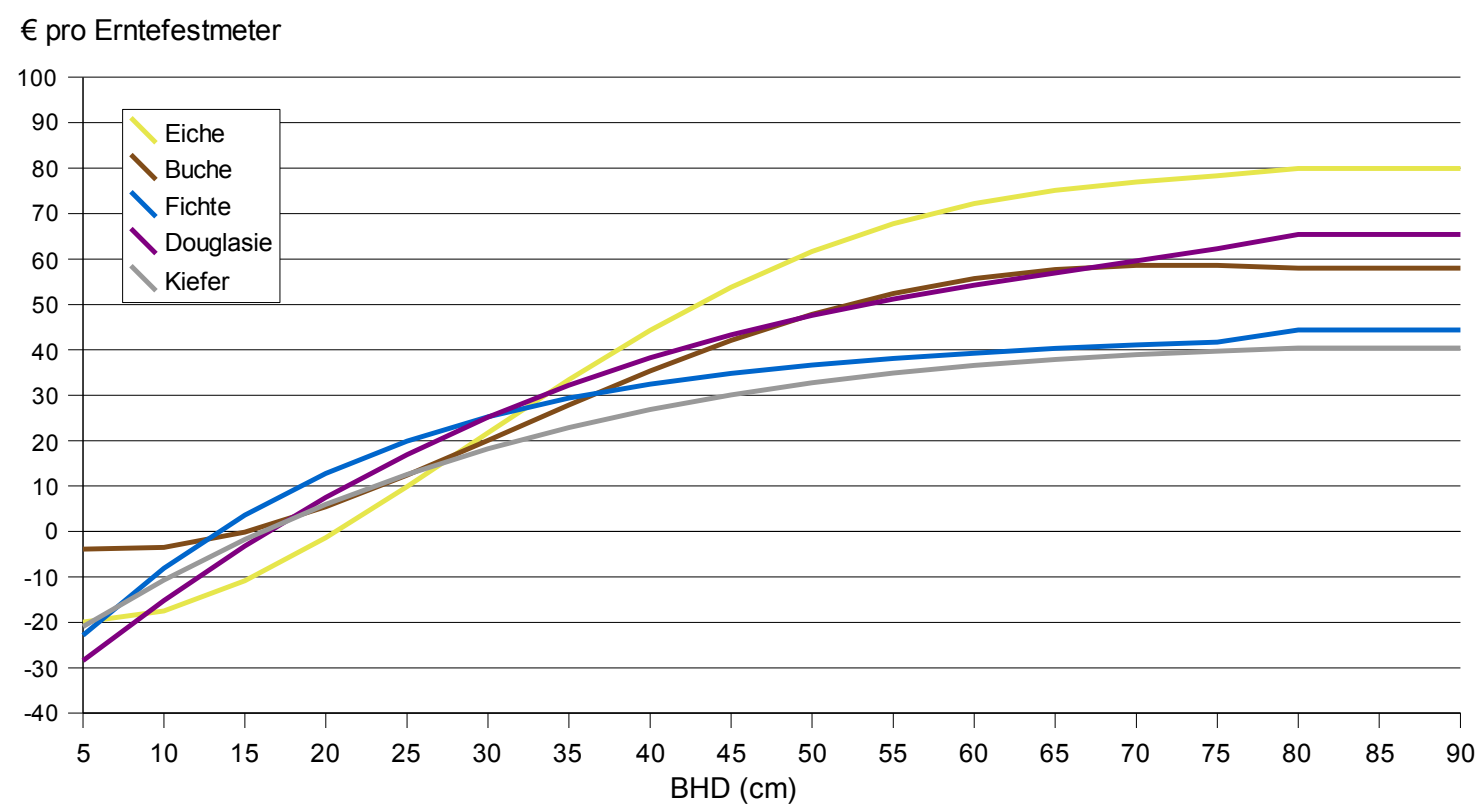

Abbildung 3.6.2.b: Erntekostenfreie Holzerlöse ${ }^{13}$ für Bäume der Wertklasse III.

\section{Vornutzungsmasse und Endnutzungsmasse}

Im Waldwachstumssimulator BWINPro werden die Informationen ausgeschiedener Bäume über die gesamte Simulationsperiode hinweg gespeichert. Sowohl Jahr und Grund des Ausscheidens (Endnutzung, Vornutzung oder natürliche Mortalität) als auch Volumen zum Zeitpunkt des Ausscheidens sind somit für jeden Simulationszeitpunkt abrufbar. Die Nutzungsmassen errechnen sich aus der Summe der Volumina der Einzelbäume. Die Daten können nach Ausscheidejahr, Art und Bestand zusammengefaßt werden.

\section{Zuwachs}

Der Zuwachs wird für jede Baumart als jährlicher Zuwachs der letzten Wachstumsperiode in Vorratsfestmetern pro Hektar angegeben. Zunächst wird der Zuwachs der letzten Wachstumsperiode errechnet und dann durch die Anzahl der Jahre geteilt. Das zugewachsene Volumen setzt sich aus dem Volumenzuwachs der im Bestand verbleibenden Bäume, dem Zuwachs der in der Periode entnommenen oder gestorbenen Bäume und dem Volumen der in der Periode eingewachsenen Bäume zusammen. Von den in der letzten Wachstumsperiode eingewachsenen Bäumen wird ein Fünftel des Gesamtvolumens des neuen Baums berücksichtigt.

Der Zuwachs eines gesamten Bestandes und der des gesamten Untersuchungsgebiets wird als jährlicher periodischer Zuwachs in Vorratsfestmetern pro Hektar errechnet. Dazu wird von dem Volumen des Bestandes zum Zeitpunkt der Betrachtung das Volumen des Bestandes am Anfang der Simulationsperiode abgezogen. Zu diesem Wert werden das entnommene Volumen (aus Endnutzung und Durchforstung) sowie das Volumen der während des Simulationszeitraums gestorbenen Bäume addiert. Das Ergebnis wird durch die Anzahl der Simulationsjahre geteilt. 


\section{Erntekostenfreie Holzerlöse der Vor- und Endnutzungen}

Wie unter dem Punkt „Abtriebswert“ beschrieben, wird jedem Baum ein erntekostenfreier Holzerlös ${ }^{13}$ zugeordnet. Ausgeschiedene Individuen erhalten den Wert, der ihnen zum Zeitpunkt ihrer Entnahme zugewiesen wurde. Da das Ausscheidejahr und der Ausscheidegrund für jeden Baum festgehalten werden, können die erntekostenfreien Holzerlöse für jedes beliebige Zeitintervall der Simlation berechnet werden.

Es wird zwischen Erlösen aus Vornutzungen und Erlösen aus Endnutzungen unterschieden. Bei der Waldumbaustrategie PROZESS ist im Laufe der Zeit mit einer qualitativen Verschlechterung der Bäume im Vergleich zu den übrigen Varianten zu rechnen, da sie Bäume ausschließlich nach ihrer Dimension fördert und die Qualität nicht berücksichtigt. Zu Beginn der Simulation wird angenommen, daß die erntekostenfreien Holzerlöse denen der anderen Varianten entsprechen. Im Laufe des Simulationszeitraums werden sie aufgrund der stetigen Qualitätsminderung mit zunehmendem Maße reduziert. Bei der Angabe der Summe der erntekostenfreien Holzerlöse wird auf eine Verzinsung der Erlöse verzichtet, um von innen auf einen durchschnittlichen Wert pro Festmeter schließen zu können. Der Entnahmezeitpunkt und der Zinssatz spielen aber für den „Holzproduktionswert“ (Definition folgt auf Seite 90) eine Rolle.

\section{Pflanzfläche}

Werden Pflanzungen vorgenommen, so wird festgehalten, in welchem Jahr die Pflanzung stattfindet, und welche Baumartengruppe mit welchem Alter auf welcher Fläche (in Hektar) gepflanzt wird. Zu jedem Zeitpunkt kann angegeben werden, welcher Pflanzaufwand im Simulationszeitraum geleistet wurde.

Tabelle 3.6.2.b: Pflanzungskosten und Pflanzungskostengrundlage (Stückzahlen, Pfanzenkosten, Pflanzkosten, Kosten für Bodenvorbereitung und Zaunbau) für die Baumartengruppen Eiche, Buche, ALh, ALn, Fichte, Douglasie und Kiefer.

\begin{tabular}{|c|c|c|c|c|c|c|}
\hline Baumartengruppe & $\begin{array}{c}\text { Stück } \\
\text { pro } \\
\text { Hektar } \\
\text { (n) }\end{array}$ & $\begin{array}{c}\text { Pflanzen- } \\
\text { kosten } \\
(€ / n)\end{array}$ & $\begin{array}{c}\text { Pflanz- } \\
\text { kosten } \\
(€ / n)\end{array}$ & $\begin{array}{c}\text { Bodenvor- } \\
\text { bereitung } \\
(€ / h a)\end{array}$ & $\begin{array}{c}\text { Zaun- } \\
\text { bau } \\
\text { (E/ha) }\end{array}$ & $\begin{array}{c}\text { Kosten } \\
\text { pro } \\
\text { Hektar } \\
\text { (€) }\end{array}$ \\
\hline Eiche & 7000 & 0,4 & 0,3 & 200 & 3000 & 8100 \\
\hline Buche & 4000 & 0,35 & 0,5 & 200 & 3000 & 6600 \\
\hline anderes Laubholz mit hoher Umtriebszeit & 3000 & 0,45 & 0,5 & 200 & 3000 & 6050 \\
\hline $\begin{array}{l}\text { anderes Laubholz mit niedriger } \\
\text { Umtriebszeit }\end{array}$ & 2500 & 0,45 & 0,5 & 200 & 0 & 2575 \\
\hline Fichte & 2000 & 0,3 & 0,5 & 200 & 0 & 1800 \\
\hline Douglasie & 1700 & 0,5 & 0,5 & 200 & 3000 & 4700 \\
\hline Kiefer & 7000 & 0,1 & 0,25 & 200 & 0 & 2650 \\
\hline
\end{tabular}

Über gängige Pflanzzahlen und Preise kann von dieser Pflanzfläche auf die aufgewendeten Pflanzungskosten geschlossen werden. Die Pflanzungskosten werden im Rahmen dieser Arbeit auf der Basis aktueller Kosten für Pflanzgut (P\&P BaumschuleN 
GмBH \& Co KG 2006) und dessen Ausbringungkosten sowie Kosten für Zaunbau (NFV 2002) berechnet. Die zu Grunde liegenden Stückzahlen sind einem Runderlaß des Niedersächsischen Ministeriums für Ernährung, Landwirtschaft und Forsten entnommen (LANDWIRTSCHAfTSministerium Niedersachsen 2004).

\section{Holzproduktionswert}

Die Summe aus erntekostenfreien Holzerlösen und Pflanzungskosten zeigt die Auswirkungen einer Waldumbaustrategie auf die Liquidität des Forstbetriebs (Finanzsphäre). Erfolgswirksam ist neben dieser Größe auch die Veränderung des Vermögens (Vermögenssphäre), die sich in der Veränderung des Abbtriebswerts eines Bestandes widerspiegelt. Ein Wert, der beide Sphären berücksichtigt kann als Anhaltspunkt für den betriebswirtschaftlichen Gesamterfolg einer Strategie dienen.

Holzproduktionswert $_{n}=\left\{\frac{A_{x+n}}{(1+i)^{n}}+\sum_{x}^{x+n}\left(\frac{D_{s}-P_{s}}{(1+i)^{s}}\right)-A_{x}\right\} \cdot\left\{\frac{i \cdot(i+1)^{n}}{(1+i)^{n}-1}\right\}$

wobei :

$n=$ Simulationszeitraum

$A=$ erntekostenfreier Abtriebswert

$x=$ Startzeitpunkt der Simulation

$i=$ Kalkulationszins (Zinssatz p/100)

$D=$ erntekostenfreie Erträge aus Entnahmen

$P=$ Kosten für Pflanzungen

$s=$ betrachtetes Jahr des Simulationszeitraums

In dieser Arbeit wird der jährliche, durchschnittliche Holzproduktionswert (siehe MöHRING ET AL. 2006) betrachtet, da in diesem Parameter beide Sphären berücksichtigt werden. Diese Erfolgsgröße ist wegen ihrer annuisierten Darstellung besonders anschaulich. Sie berücksichtigt durch Diskontierung die Zeitpunkte, zu denen Erlöse und Kosten anfallen. Es wird ein Zinssatz von 2\% unterstellt (siehe MoOg UND BRABÄNDER 1994). Ein weiterer Vorteil dieses Werts ist, daß er auch für die Betrachtung von Teilen der Umtriebszeit aussagekräftig ist. Die anfallenden Pflanzungskosten können berücksichtigt oder aus der Betrachtung ausgeschlossen werden.

\section{Durchmischungskoeffizient}

Bei der Betrachtung der Bestandestypenverteilung hinsichtlich der Landschaftsdiversität ist vor allem die Information von Interesse, wie groß- oder kleinflächig „geblockt“ ähnliche Bestandestypen in der betrachteten Waldlandschaft auftreten. Geographische Informationssysteme bieten rasterzellenbasierte Nachbarschaftsanalysen als geeignete Lösungsansätze an. Hierzu wird die digitale Bestandestypenkarte im Geographischen Informationssystem in eine Rasterkarte umgewandelt. Mit den gängigen geostatistischen Analysewerkzeugen können anschließend die Auswertungen im GIS durchgeführt werden. Im Rahmen dieser Arbeit wurde die von Tomlin beschriebene Nachbarschaftsanalyse „FocalVariety“ verwendet (Tomlin 1990), die zum Beispiel in GRASS unter dem Namen r.neighbours (method diversity) aufgerufen werden kann (siehe GRASS Development 
). Sie gibt an, wieviele verschiedene Analyseklassen innerhalb eines Analysefensters gefunden werden, ordnet diesen Wert der zentralen Zelle des Analysefensters zu und schreibt inn in die korrespondierende Zelle einer Ergebniskarte.

Die digital vorliegende Bestandestypenkarte wird für die Nachbarschaftsanalyse in eine Rasterkarte mit einer Rasterzellengröße von $20 \mathrm{~m} \times 20 \mathrm{~m}$ umgewandelt. Auf diese Rasterkarte wird ein Analysefenster von $3 \times 3$ Zellen angewendet. Ein Rasterzellenwert der Ergebniskarte von 1 sagt aus, daß die korrespondierende Zelle der analysierten Karte vollständig von Zellen umgeben ist, die dem gleichen Bestandestypen angehören. Der höchstmögliche Wert 8 wird erreicht, wenn eine Rasterzelle von acht verschiedenen Bestandestypen umgeben ist.

Die Ergebniskarte kann folgendermaßen interpretiert werden: Ein hoher Anteil niedriger Werte repräsentiert eine hohe Clusterung der Analyseklassen (Bestandesstypen) und läßt somit auf eine relativ geringe landschaftliche Vielfalt schließen. Der Durchschnittswert aller Zellen wird im folgenden als Durchmischungskoeffizient bezeichnet. Seine Höhe wird als Maß für die landschaftliche Vielfalt herangezogen. Die Aussagekraft des Werts wird erhöht, indem man inn mit dem höchst möglichen Durchmischungskoeffizienten vergleicht.

Dieser wird durch die Annahme der größtmöglichen landschaftlichen Vielfalt ermittelt. Die größtmögliche landschaftliche Vielfalt unter Beibehaltung der Bestandesgrenzen ist gegeben, wenn alle Bestände an Bestände mit einem Bestandestyp grenzen, die nicht ihrem eigenen entsprechen. Diese Situation kann simuliert werden, in dem jedem Bestand statt des tatsächlichen Bestandestyps eine fortlaufende Nummer zugeordnet wird.

\section{Index-A}

Der Index-A nach Pretzsch (Pretzsch 1996) baut auf dem Index-H von Shannon und Weaver (siehe SHANnoN 1948) auf, die diesen Index im Zusammenhang von Kommunikationstechnologien entwickelten. Er berücksichtigt die Anzahl der Arten und ihre relative Häufigkeit pro Höhenschicht. So wird neben der Artendiversität gleichzeitig auch die Raumbesetzung der Arten im Waldbestand quantifiziert. Die Bildung von Höhenschichten richtet sich laut Pretzsch nach der Höhe des Einzelbaums im Vergleich zur Maximalhöhe des Bestandes. Da für Simulationen der Bestände keine Maximalhöhe definiert ist, wird bei der Herleitung des Index-A auf die intern verwendete Bestandesschichteneinteilung zurückgegriffen, die dem Modell von Pretzsch sehr nah kommt (siehe Glossar).

Für jeden Waldbestand wird der Index-A nach Pretzsch automatisch berechnet. Niedrige Indexwerte ergeben sich in einschichtigen Reinbeständen, die höchsten Werte werden in stark strukturierten Mischbeständen erreicht.

\section{Totholzvolumen}

Das Totholzvolumen errechnet sich aus dem Volumen aller während des Simulationszeitraums durch natürliche Mortalität aus dem Bestand ausgeschiedenen Bäume in Vorratsfestmetern je Hektar. Bei der Nutzung verbliebenes Holz oder 
Kronenmaterial, Bäume mit einem BHD unter $7 \mathrm{~cm}$ sowie absterbende Äste können in der Simulation nicht berücksichtigt werden.

Eine Totholzbildungsrate kann durch die Division der angefallenen Masse an Totholz durch die Anzahl der Jahre, in denen es angefallen ist, hergeleitet werden. Sie kann baumartenspezifisch oder bestandesbezogen berechnet werden. Eine Zersetzungsrate ist nicht hinterlegt.

\section{Habitatbaumvolumen}

Die Volumina der zum betrachteten Zeitpunkt als Habitatbaum markierten Individuen des Bestandes werden aufsummiert und mit der simulierten Flächengröße gewichtet. Die Summe der Habitatbaumvolumina wird in Vorratsfestmetern pro Hektar ausgegeben. Sie sagt aus, wieviel Holz zum betrachteten Zeitpunkt durch Unterschutzstellung der Nutzung entzogen ist.

\section{Zielerreichungsprozent}

Das Zielerreichungsprozent gibt an, zu wieviel Prozent die Kronenschirmflächenanteile, die mit dem Zieltyp angestrebt werden, im Bestand verwirklicht sind. (Es sagt nichts über den Gesamterfolg der Strategie aus.) Die zu erreichenden Werte für Haupt- und Nebenbaumarten werden der Metadatenbank entnommen und mit dem vorhandenen Kronenschirmflächenanteil der Baumart (aus Bäumen $>7 \mathrm{~cm}$ BHD) verglichen.

Dieses Vorgehen kann durch das folgende Beispiel veranschaulicht werden: Der Zielbestand soll aus 60\% Buche und 30\% Fichte bestehen, der aktuelle Bestand weist aber eine Kronenschirmflächenverteilung von 90\% Buche zu 10\% Fichte auf. Die Buche übererfüllt in diesem Beispiel die vorgegebenen 60 Prozentpunkte, die Fichte erfüllt nur zehn der geforderten 30 Prozentpunkte. Von den 90 vorgegebenen Prozentpunkten (60 für Buche +30 für Fichte), sind demnach 70 (60 für die Buche +10 für die Fichte) durch die derzeitige Bestockung erfüllt. Das Zielerreichungsprozent beträgt in diesem Fall $77,8 \%$ (70/90*100).

\section{Verteilung der Bestandestypen}

Unter einem Bestandestyp werden Bestände mit gleicher oder ähnlicher Baumartenzusammensetzung zusammengefaßt (nach NiEDERSÄCHSISCHE LANDESFORSTVERWALTUNG 1987). Es wird zwischen Rein- und Mischbeständen unterschieden. Als Reinbestand gilt ein Bestand, dessen Hauptbaumart mindestens 90\% der Summe der Kronenschirmflächen des Bestandes ausmacht. Kommen die Nebenbaumarten gemeinsam auf eine Kronenschirmflächensumme von über $10 \%$, so spricht man von einem Mischbestand. Eine Art bestimmt die Bestandestypenbezeichnung mit, wenn sie mindestens $10 \%$ der Kronenschirmfläche des Bestandes einnimmt. Die deutlich überwiegenden Haupt- und Mischbaumarten eines Bestandes bestimmen seinen Mischbestandestyp. 
Auf der Landschaftsebene wird untersucht, zu welchen Teilen das Untersuchungsgebiet welchem Bestandestyp angehört. Das Ergebnis kann mit der angestrebten Zieltypenverteilung verglichen werden.

\section{Ergebnisse und Diskussion}

\subsection{Beispielbestände}

\subsubsection{Beispiele für die Anwendung der Durchforstungs- algorithmen}

In den Beispielbeständen 1, 2 und 3 stehen in allen vier Simulationsvarianten (LÖWE, PNV, ERTRAG und PROZESS) in den nächsten vierzig Jahren verschiedene Durchforstungsmaßnahmen an; die Varianten LÖWE, PNV und ERTRAG sehen in diesem Zeitraum Pflanzungen vor. Die durchgeführten Eingriffe hängen sowohl von den Maßnahmenketten (siehe Kapitel 3.5.1 bis 3.5.4), als auch von den Rahmenwerten der jeweiligen Strategie (siehe auch Tabellen 3.5.a und 3.5.b) ab. Den Beispielbeständen wurden für die einzelnen Simulationsvarianten im Untersuchungsgebiet häufig vorkommende Zieltypen zugeordnet, für die jeweils spezifische Baumartenmischungen festgelegt sind. Nur in der Variante PROZESS wird, gemäß der Definition der Simulationsvariante, auf eine solche Zielsetzung verzichtet.

Für jeden der vier Bestände werden die Auswertungsparameter zum Startzeitpunkt und nach Ablauf der vier Simulationen in Tabellenform vorgestellt (siehe Tabellen 4.1.1.a, 4.1.1.b, 4.1.1.c und 4.1.2.a). Auf eine Darstellung der Durchmesserstruktur wurde verzichtet. Die Tabellen können auf die landschaftsbezogenen Parameter „Durchmischung auf Landschaftsebene“ und „Verteilung der Bestandestypen“ (siehe Tabelle 3.6.2.a) keinen Bezug nehmen, da es sich um Einzelbestände handelt. Abbildungen zeigen, wie sich das Erscheinungsbild der Bestände in den vierzig Simulationsjahren ändert.

\section{Bestand 1}

Besonders deutlich wird der Effekt der verschiedenen Behandlungen auf die Bestände, in den Unterschieden der Endzustände der vier Varianten in Bestand 1 (siehe Abbildung 4.1.1.a). Die Entwicklung des Bestandes wird daher anhand der Abbildung 4.1.1.b näher betrachtet. In dieser zweiten Darstellung werden die Unterschiede der Durchforstungseingriffe und Pflanzungsmaßnahmen der vier Varianten mit Hilfe von Stammverteilungsplänen verdeutlicht. Bei der Auswertung der Abbildung ist zu beachten, daß bei den Varianten LÖWE und PNV alle fünf Jahre Eingriffe stattfinden, aber nur Stammverteilungspläne im Abstand von zehn Jahren abgebildet sind.

Der Grund für die charakteristische Entwicklung des Bestandes 1 ist, daß im Unterschied zu den Beispielbeständen 2 und 3 die gepflanzte Verjüngung noch während des Simulationszeitraums in den Bestand einwächst. 


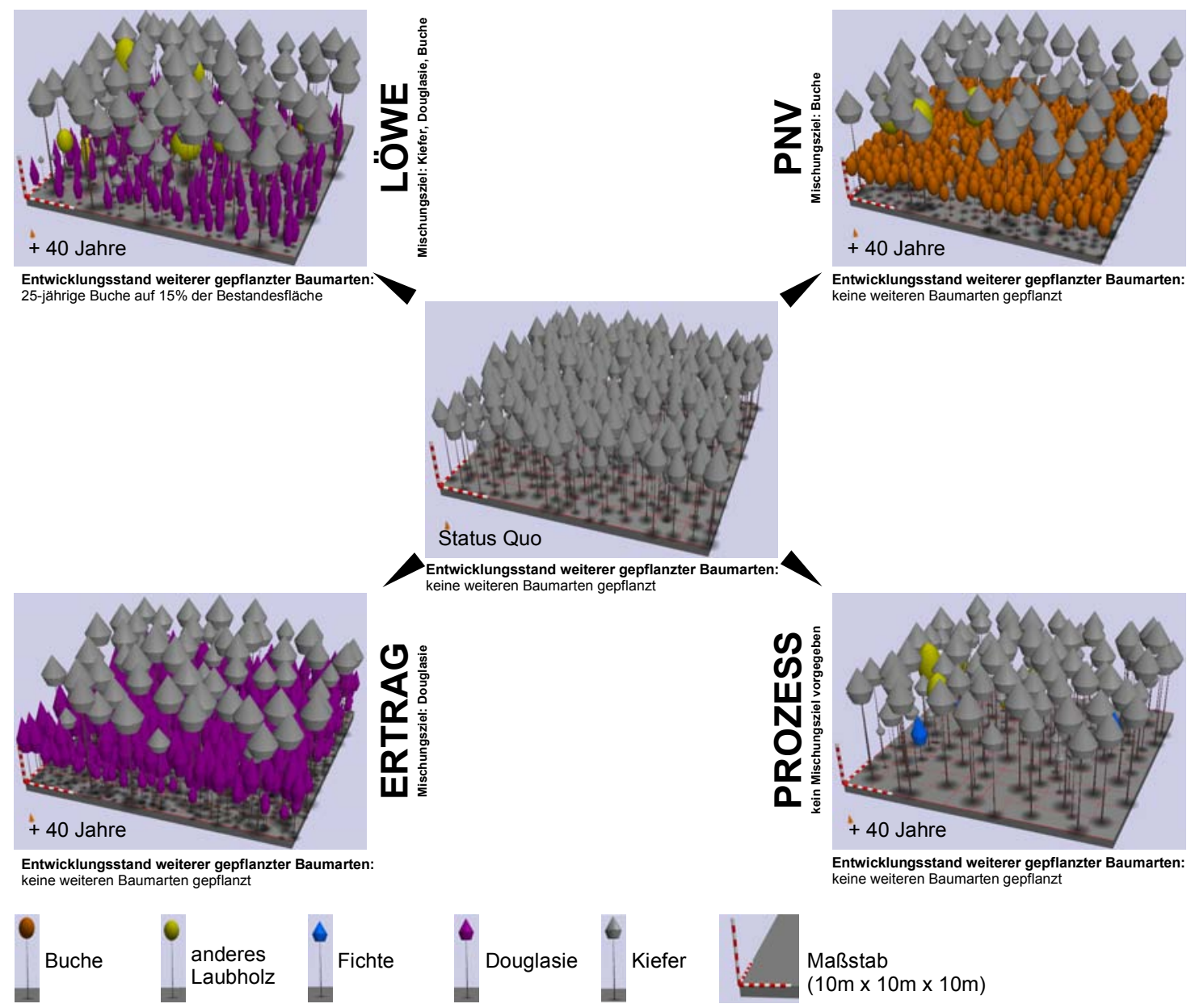

Abbildung 4.1.1.a: Simulationsergebnisse der Szenarien LÖWE, PNV, ERTRAG und PROZESS nach vierzigjähriger Entwicklung eines Bestandes aus fünfzigjähriger Kiefer (Bestand 1 aus Tabelle 3.2.2.a)

Fehlen Baumarten oder erhebliche Anteile einiger Arten zur angestrebten Baumartenmischung, so wird eine Pflanznotwendigkeit registriert. Wenn Pflanzungen Teil des Waldbaukonzepts sind, wird dann nach jedem Eingriff geprüft, ob die Bedingungen für die Ausführung der simulationsspezifischen Pflanzroutinen gegeben sind. Da die Variante PROZESS keinen Zieltyp anstrebt und auch keine Pflanzungen vorsieht, wird in diesem Simulationslauf keine Baumart durch Pflanzung eingebracht. Für die Variante LÖWE wird ein Kiefern-/ Douglasien-/ Buchenbestand, für die Variante PNV ein Buchenbestand, für die Variante ERTRAG ein Douglasienbestand angestrebt. Bedingung für Durchführung der Pflanzung in den Varianten LÖWE, PNV und ERTRAG ist, daß die Überschirmungsverhältnisse dies zulassen und mindestens $70 \%$ der Bäume der führenden Schicht ihren Zieldurchmesser zu 80\% erreicht haben. Im Gegensatz zu den Simulationsläufen der Bestände 2 und 3 werden diese Bedingungen im Bestand 1 schon im Jahr 0 der Simulation erfüllt. Nach dem ersten Durchforstungseingriff bei der Variante ERTRAG wird ein Teil (80\%) des geforderten Douglasienanteils gepflanzt; im zehnten Jahr der Simulation werden die verbliebenen Anteile (20\%) eingebracht (siehe Abbildung 4.1.1.b). 
Da der Zieldurchmesser für Kiefer in den Varianten PNV und LÖWE höher liegt und die Überschirmung nach dem ersten Eingriff noch zu dicht steht, finden die Pflanzungen in diesen beiden Varianten erst später statt. In der Variante PNV wird im fünften Simulationsjahr Buche, in der Variante LÖWE werden im zehnten Simulationsjahr Buche und Douglasie gepflanzt. Die Varianten LÖWE und PNV lassen neben dem Einwuchs aus Pflanzung auch Raum für den Einwuchs natürlicher Verjüngung, da nur auf einem Teil der Bestandesfläche gepflanzt wird. In der Variante PROZESS wachsen besonders zahlreiche Baumarten aus natürlicher Verjüngung ein, da die natürliche Verjüngung keine Konkurrenz aus Pflanzungen hat. Der Einwuchs setzt sich in dieser Variante aus den Baumarten Sandbirke, Moorbirke, Eberesche, Fichte und Kiefer zusammen. Die in der Variante ERTRAG auf der ganzen Bestandesfläche durchgeführte Pflanzung verhindert weitgehend das Aufkommen natürlicher Verjüngung, so daß der Einwuchs dort fast ausschließlich auf Pflanzungen zurückzuführen ist.

Neben den Unterschieden, die sich durch die Pflanzungsmaßnahmen ergeben, führen simulationsspezifische Durchforstungseingriffe zu charakteristischen Ergebnissen, die auch auf die Bestandesentwicklung der Bestände 2 und 3 übertragbar sind. Die Maßnahmenketten für LÖWE, PNV und ERTRAG sehen die Auswahl permanenter ZBäume vor (siehe Kapitel 3.5.1 bis 3.5.4). Es werden lediglich im LÖWE-Simulationslauf permanente Z-Bäume markiert (siehe Abbildung 4.1.1.b), da nur in diesem Fall die mit den Rahmenwerten gesetzten Kriterien für die Auswahl permanenter Z-Bäume erfüllt werden. Permanente Z-Bäume werden markiert, wenn sich im Bestand Bäume einer im Zieltyp vorgesehenen Art befinden, deren Höhen über der geforderten Mindesthöhe liegen. Aus dem gegebenen Kiefernbestand können nur für die Variante LÖWE permanente Z-Bäume ausgewählt werden, da nur im Zieltyp für die Variante LÖWE (Kiefern-/ Douglasien-/ Buchenbestand) die Baumart Kiefer vorkommt (siehe Jahr 0 in Abbildung 4.1.1.b). Die gegen Ende der Simulationsläufe einwachsenden Bäume aus Pflanzungen erreichen nicht die zur Z-Baum-Auswahl geforderte Mindesthöhe. Daher werden bis zum Ende der Simulationsläufe in keiner der Varianten weitere permanente Z-Bäume ausgewählt.

Die Auswahl der temporären Z-Bäume im Jahr 0 führt in den Varianten PNV, ERTRAG und PROZESS zu identischen Ergebnissen. Erst bei der Freistellung der ausgewählten Bäume unterscheiden sich die drei Varianten. Die markierten Kiefern werden in den Varianten PNV und ERTRAG stark, in der Variante PROZESS dagegen schwach freigestellt. Die maximale Durchforstungsmenge der Variante ERTRAG erlaubt mit $100 \mathrm{Vfm} / \mathrm{ha}$ einen stärkeren Eingriff als die anderen Varianten mit $70 \mathrm{Vfm} / \mathrm{ha}$. In der Variante LÖWE wird die maximale Durchforstungsmenge schon bei der Freistellung der permanenten Z-Bäume erreicht. Zu einer Freistellung der temporären Z-Bäume kommt es daher nicht. Die minimale Durchforstungsmenge liegt bei der Variante ERTRAG mit $75 \mathrm{Vfm} /$ ha deutlich höher als die in den drei anderen Varianten geforderten $35 \mathrm{Vfm} / \mathrm{ha}$. Nach dem zehnten Simulationsjahr kann in der Variante ERTRAG, nach dem fünfundzwanzigsten Simulationsjahr in der Variante LÖWE die geforderte Mindestmasse nicht mehr erreicht werden; es finden in diesen Läufen bis zum Ende der Simulation keine weiteren Durchforstungen statt. 

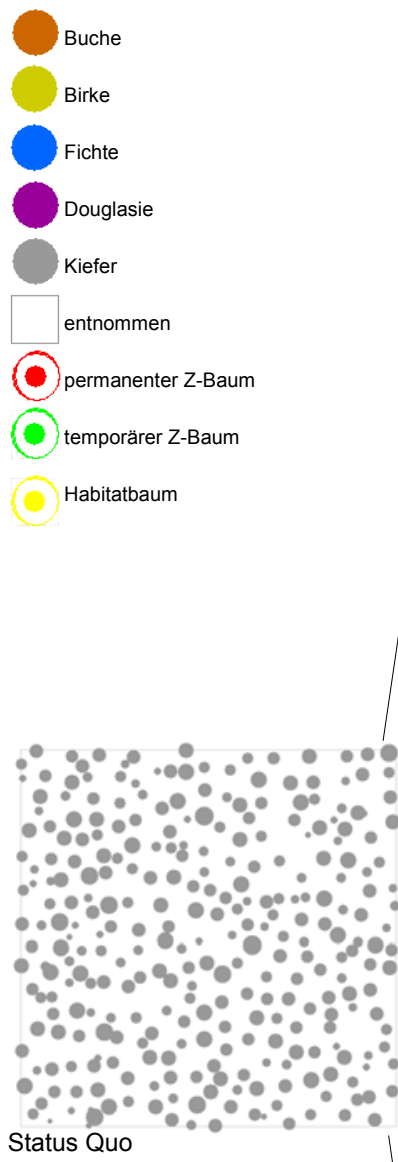

Status Quo

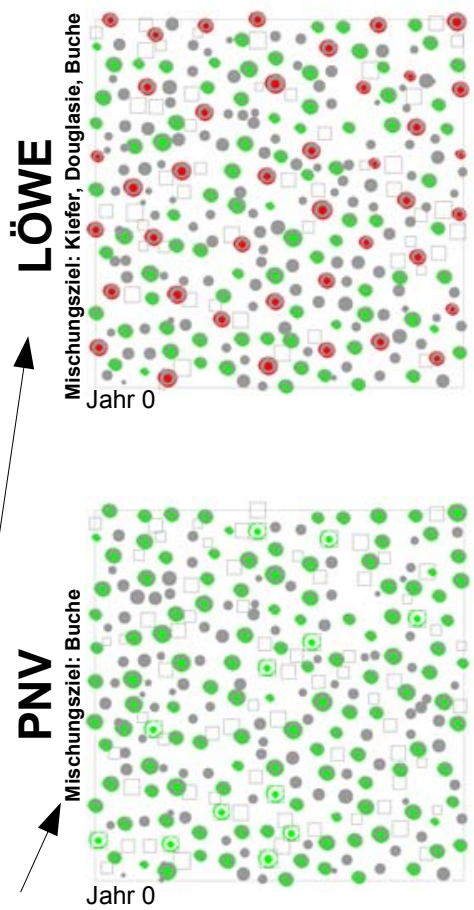

Jahr 0

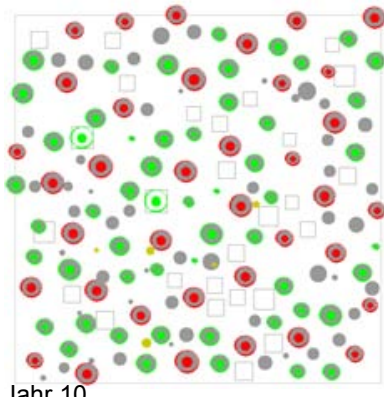

Jahr 10

Pflanzmaßnahmen:

Buche auf $30 \%$ der Bestandesfläche Douglasie auf $30 \%$ der Bestandesfläche

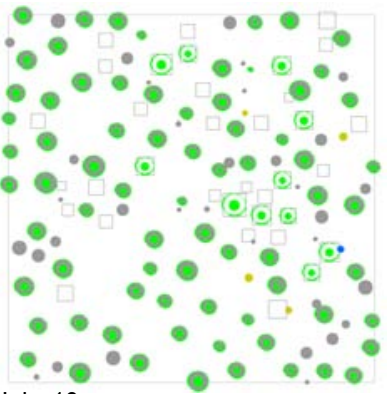

Jahr 10

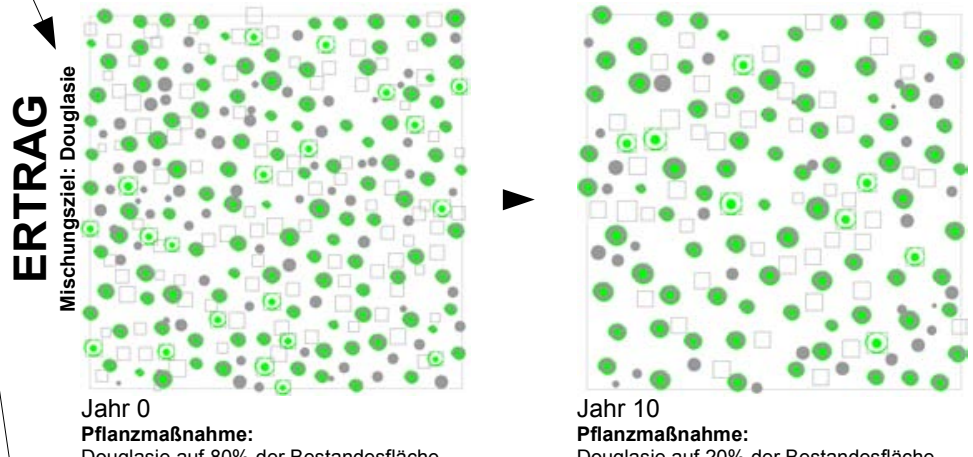

Douglasie auf $20 \%$ der Bestandesfläche

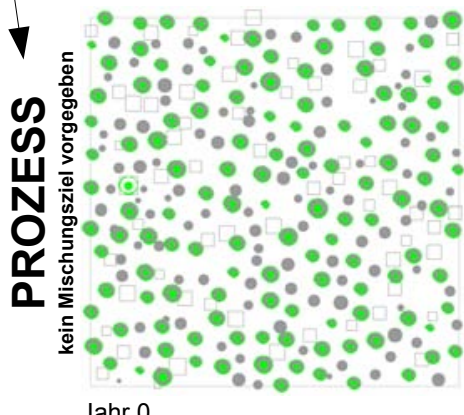

Abbildung 4.1.1.b: Die vierzigjährige Entwicklung eines Bestandes aus fünfzigjähriger Quo und der Zwischenergebnisse der Szenarien LÖWE, PNV, ERTRAG und (auch für die Strategien LÖWE und PNV, die zwei Eingriffe im Jahrzehnt vorsehen). Stämme werden mit einer dreifach vergrößerten Grundfläche dargestellt. 

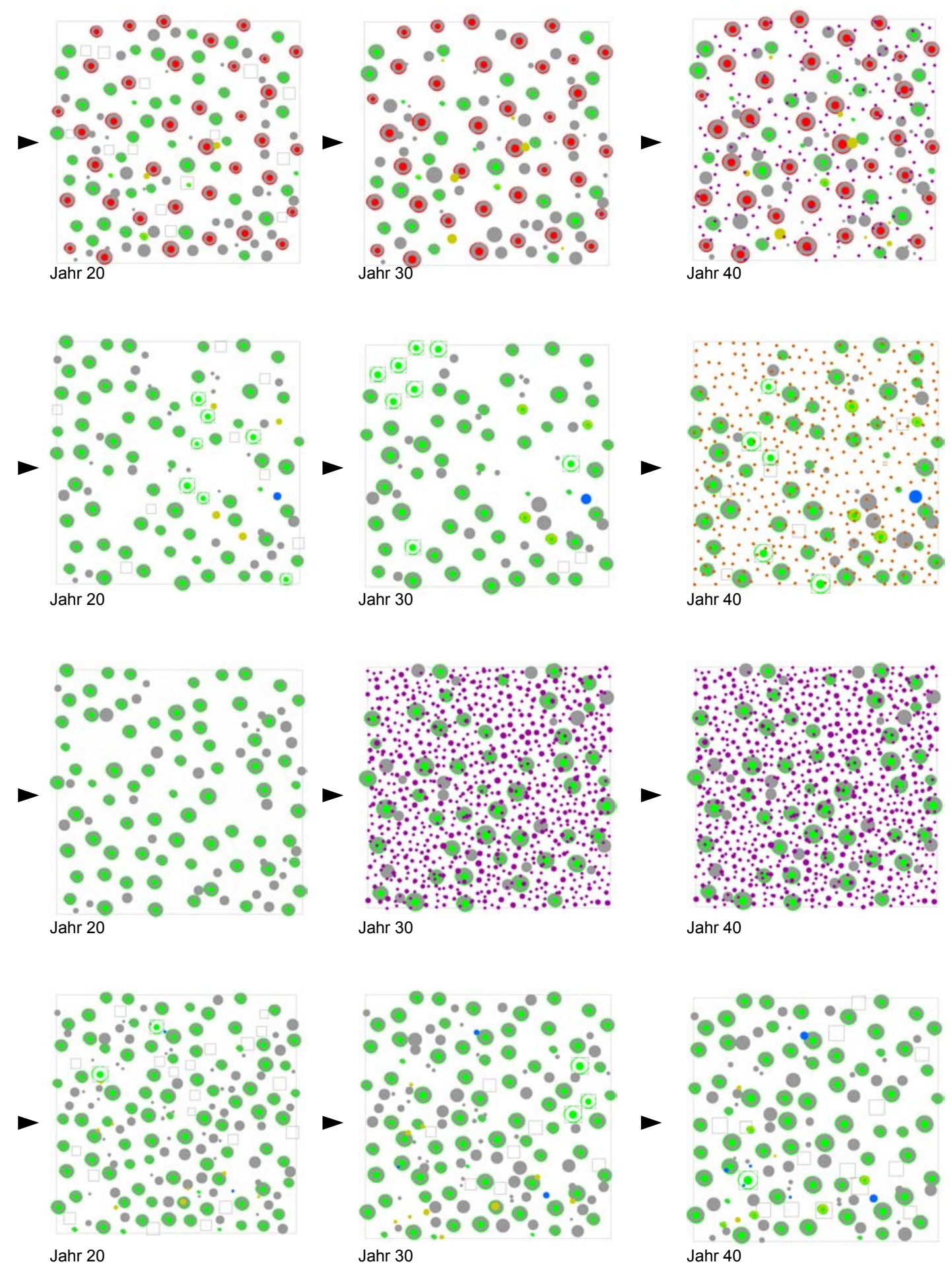

Kiefer (Bestand 1 aus Tabelle 3.2.2.a) anhand von Stammverteilungsplänen des StatusPROZESS. Die Einzelbilder sind im Abstand von zehn Jahren festgehalten worden. Die 
Die unterschiedliche Entwicklung der Bestände wirkt sich auch auf die Auswertungsparameter aus, die in Tabelle 4.1.1.a zu finden sind. Die Art der Eingriffe spiegelt sich direkt in der Vornutzungsmasse wider, die als Summe der Vornutzungsmassen über vierzig Jahre angegeben wird. Endnutzungen finden nicht statt. Die größte Menge $(273,8 \mathrm{Vfm} / \mathrm{ha})$ wird, bedingt durch häufige Eingriffe mit hohem Freistellungsgrad, in der Variante PNV entnommen. Obwohl nur in den ersten zwanzig Jahren der Simulation in der Variante ERTRAG Durchforstungseingriffe stattfinden, wird in dieser Variante mit 244,1 Vfm/ha die zweithöchste Masse über die simulierten vierzig Jahre entnommen. Die Varianten PROZESS und LÖWE liegen mit 213,7 und 211,3Vfm/ha deutlich darunter.

Tabelle 4.1.1.a: Übersicht über die Ergebnisse der Simulation des Bestandes 1 anhand der Auswertungsparameter.

\begin{tabular}{|c|c|c|c|c|c|c|}
\hline Kriterium & Parameter & Status Quo & $L \ddot{O} W E$ & $P N V$ & ERTRAG & PROZESS \\
\hline \multirow[t]{2}{*}{$\begin{array}{l}\text { Forstliche } \\
\text { Ressourcen }\end{array}$} & $\begin{array}{l}\text { Derbholzvorrat } \\
(\mathrm{Vfm} / \mathrm{ha})\end{array}$ & 255,5 & 273,3 & 188,5 & 366,6 & 270,9 \\
\hline & Abtriebswert (E/ha) & 952 & 4030 & 3001 & 2609 & 4275 \\
\hline \multirow[t]{7}{*}{$\begin{array}{l}\text { Produktions- } \\
\text { funktion }\end{array}$} & $\begin{array}{l}\text { Vornutzungsmasse } \\
\text { (Vfm/ha) }\end{array}$ & keine Angabe & 211,3 & 273,8 & 244,1 & 213,7 \\
\hline & $\begin{array}{l}\text { Endnutzungsmasse } \\
\text { (Vfm/ha) }\end{array}$ & keine Angabe & 0 & 0 & 0 & 0 \\
\hline & $\begin{array}{l}\text { Zuwachs } \\
(\text { Vfm/ha/a) }\end{array}$ & keine Angabe & 7,2 & 6,0 & 10,4 & 7,3 \\
\hline & $\begin{array}{l}\text { erntekostenfreie } \\
\text { Holzerlöse } \\
\text { Vornutzungen } \\
(\text { (E/ha) }\end{array}$ & keine Angabe & 1823 & 2426 & 2018 & 2122 \\
\hline & $\begin{array}{l}\text { erntekostenfreie } \\
\text { Holzerlöse } \\
\text { Endnutzungen } \\
(\text { (E/ha) }\end{array}$ & keine Angabe & 0 & 0 & 0 & 0 \\
\hline & $\begin{array}{l}\text { Pflanzfläche } \\
\text { (\% der Bestandesfläche) }\end{array}$ & keine Angabe & 60 & 80 & 100 & 0 \\
\hline & $\begin{array}{l}\text { Holzproduktionswert } \\
(\boldsymbol{E} / \mathrm{ha} / \mathrm{a})\end{array}$ & keine Angabe & -23 & -119 & -76 & 84 \\
\hline \multirow{3}{*}{$\begin{array}{l}\text { Biologische } \\
\text { Diversität }\end{array}$} & Index- $A$ & 0,50 & 0,95 & 0,66 & 0,85 & 0,78 \\
\hline & $\begin{array}{l}\text { Totholzvolumen } \\
\text { (Vfm/ha) }\end{array}$ & keine Angabe & 59,1 & 33,1 & 59,8 & 63,2 \\
\hline & $\begin{array}{l}\text { Habitatbaumvolumen } \\
(\mathrm{Vfm} / \mathrm{ha})\end{array}$ & keine Angabe & 0 & 0 & 0 & 0 \\
\hline Zielerreichung & $\begin{array}{l}\text { Zielerreichungsprozent } \\
\text { (\%) }\end{array}$ & $\begin{array}{l}\text { LÖWE: } 55 \\
\text { PNV: } 10 \\
\text { ERTRAG: } 0 \\
\text { PROZESS: } \mathrm{x}\end{array}$ & 75 & 69 & 72 & $x$ \\
\hline
\end{tabular}

Die Summe der erntekostenfreien Holzerlöse hängt von den entnommenen Mengen und der Durchmesserstruktur der entnommenen Bäume ab. Am höchsten ist die Summe mit 2426€/ha in der Variante PNV, dann folgen PROZESS (2122€/ha), ERTRAG (2018€/ha) und LÖWE (1823€/ha). Interessant ist, daß trotz des höheren Entnahmevolumens der Variante ERTRAG im Vergleich zur Variante PROZESS die erntekostenfreien Holzerlöse dieser Variante durch den geringeren Erlös pro Festmeter niedriger liegen. Der Grund liegt 
in den frühen starken Eingriffen der Variante ERTRAG, durch die auf spätere Durchforstungseingriffe verzichtet werden kann. Der durchschnittliche Durchmesser der ausscheidenden Bäume liegt unter dem der Variante PROZESS, die bis zum Ende der Simulation Durchforstungseingriffe mit geringer Stärke durchführt. Verzinst man die erntekostenfreien Holzerlöse der beiden Varianten mit $2 \%$, so wird der geringere Festmetererlös durch den früheren Eingriffszeitpunkt wieder ausgeglichen, und die Erlöse der Variante ERTRAG liegen über denen der Variante PROZESS.

Die anfallende Totholzmenge aus abgestorbenen Bäumen über $7 \mathrm{~cm}$ BHD zeigt, daß die Varianten LÖWE, ERTRAG und PROZESS wesentlich mehr Bäume bei Durchforstungen im Bestand belassen, die durch natürliche Mortalität vergehen, als die Variante PNV. In diesem Simulationslauf liegt die aufsummierte Totholzmasse über den Simulationszeitraum von vierzig Jahren bei $33,1 \mathrm{Vfm} / \mathrm{ha}$. Die größte Mortalität ist in der Variante PROZESS mit $63,2 \mathrm{Vfm} / \mathrm{ha} \mathrm{zu}$ beobachten. Sie liegt dort allerdings nicht wesentlich höher als bei den Varianten LÖWE $(59,1 \mathrm{Vfm} / \mathrm{ha})$ und ERTRAG $(59,8 \mathrm{Vfm} / \mathrm{ha})$.

Mit der Entnahme von Bäumen wird Einfluß auf die Entwicklung der verbleibenden Bäume und damit auf die Entwicklung des Zuwachses und des Derbholzvorrates genommen. Auch die Menge an anfallendem Totholz fließt in diese beiden Auswertungsparameter mit ein. In der Variante PNV wird - im Gegensatz zu den anderen drei Varianten - so stark in den Bestand eingegriffen, daß der Derbholzvorrat von $255,5 \mathrm{Vfm} / \mathrm{ha}$ auf $188,5 \mathrm{Vfm} / \mathrm{ha}$ sinkt. Diese Absenkung wirkt sich auch auf den Zuwachs aus, der mit 6Vfm/ha/a deutlich unter den Werten der anderen drei Varianten liegt. Die Variante PROZESS kommt auf einen Zuwachs von 7,3Vfm/ha pro Jahr, der Derbholzvorrat am Ende der Simulation liegt bei $270,9 \mathrm{Vfm} / \mathrm{ha}$. Die Variante LÖWE liegt mit 7,2Vfm/ha knapp unter diesem Wert. Der Derbholzvorrat wächst auf $273,3 \mathrm{Vfm} / \mathrm{ha}$. In den beiden Varianten spielen die wenigen Einwachser aus Naturverjüngung keine große Rolle. Den höchsten jährlichen Zuwachs hat die Variante ERTRAG mit 10,4Vfm/ha/a. Der hohe Zuwachs führt trotz hoher Entnahmen zum umfangreichsten Derbholzvorrat am Ende des Simulationszeitraums (366,6Vfm/ha). Besonders der Einwuchs der gepflanzten Douglasien trägt zu diesem Ergebnis bei.

Neben dem Zuwachs beeinflußt der Einwuchs der gepflanzten Douglasie in der Variante ERTRAG auch den Abtriebswert des verbliebenen Bestandes stark. Mit 2609€/ha liegt er deutlich unter den Werten der anderen drei Varianten, da ein Großteil der Douglasien bei einer Entnahme negative Erlöse bringen würde. Der negative Effekt der jungen Douglasien wird bei der Betrachtung des durchschnittlichen Erlöses pro Festmeter deutlich. Er beträgt in der Variante ERTRAG mit ca. $7 €$ weniger als die Hälfte der Werts für den FestmeterErlös der anderen Varianten. In der Variante PNV kommt man zum Beispiel auf knapp 16€ pro Festmeter. Einerseits ist der Anteil der Buchen in der Variante PNV geringer als der Anteil der Douglasien in der Variante ERTRAG, andererseits sinken die Erlösfunktionen der Buche bei geringen Durchmessern nicht so stark in den negativen Bereich wie die der Douglasie (siehe Abbildungen 3.6.2.a und 3.6.2.b). In diesem Zusammenhang stellt sich die Frage, inwieweit die Einbeziehung von Bäumen, die bei klassischer Nutzung negative Erlöse erzielen würden, für die vergleichende Betrachtung von zwei Zuständen sinnvoll ist. Alternativ könnte man die Annahme treffen, die Biomasse dieser Bäume zu Hackschnitzeln zu verarbeiten oder die Bäume gegen einen geringen Betrag an Selbstwerber zu verkaufen. In diesen Fällen wären die Abtriebswerte enger mit den 
Vorräten und der Durchmesserstruktur der führenden Kiefer gekoppelt als mit der einwachsenden Baumart.

In den Varianten PNV, LÖWE und PROZESS werden die Abtriebswerte nur wenig durch eingewachsene Bäume beeinflußt. Sie korrelieren stark mit dem Derbholzvorrat. So kommt die Variante PROZESS wegen der hohen Massenakkumulation auf einen Abtriebswert von 4275€, LÖWE auf 4030€ und PNV wegen der Absenkung des Vorrates lediglich auf $3001 €$ pro Hektar.

Durch die Pflanzungsmaßnahmen in den Varianten LÖWE, PNV und ERTRAG fällt der Holzproduktionswert dieser Varianten in den negativen Bereich. Bei der Variante PROZESS, die keine Pflanzungen vorsieht, liegt er bei $84 €$ pro Hektar und Jahr. Läßt man die Pflanzungskosten bei der Berechnung des Holzproduktionswerts außer acht, so liegt er in der Variante LÖWE bei $79 €$, in der Variante PNV bei $77 €$ und in der Variante ERTRAG bei $61 €$ pro Hektar und Jahr. Die Verzerrung der Abtriebswerte durch die Annahme negativer Holzerlöse kommt natürlich auch bei der Berechnung des Holzproduktionswerts zum Tragen. Gerade die Variante ERTRAG wird durch den Einfluß der eingewachsenen Douglasie negativ beeinflußt.

Bezieht man die Pflanzungskosten in den Holzproduktionswert mit ein, so wird der Wert in der Variante PNV am stärksten negativ beeinflußt, da in dieser Variante auf $100 \%$ der Fläche die kostenintensive Buche (siehe Pflanzungskosten in Tabelle 3.6.2.b) gepflanzt wird. Der Holzproduktionswert beträgt $-119 €$ pro Hektar und Jahr. Zwar arbeitet die Variante ERTRAG mit der kostengünstigeren Douglasie, jedoch beeinflußt ihre Pflanzung auf ganzer Fläche den Holzproduktionswert immer noch stark; er liegt für diese Variante bei $-76 €$ pro Hektar. Die Variante LÖWE wird wegen des geringeren Flächenanteils, auf dem Pflanzungen durchgeführt wurden und wegen der Mischung aus Douglasie und Buche von der Pflanzung weniger beeinflußt. Dennoch liegt der Holzproduktionswert mit $-23 €$ pro Hektar und Jahr im negativen Bereich.

Der Index-A beträgt zum Startzeitpunkt der Simulation 0,50. Er ist in diesem relativ homogenen Reinbestand nicht gleich Null, da die Bäume des Kiefernkollektivs rechnerisch zwei Höhenschichten zugeordnet werden. In allen vier Simulationsläufen steigt der IndexA bis zum Ende des Simulationszeitraums an. Am höchsten ist er mit 0,95 in der Variante LÖWE. Dort wachsen neben den zwei gepflanzten Baumarten (Buche und Douglasie) auch noch drei weitere Baumarten (Sandbirke, Moorbirke und Eberesche) während des Simulationszeitraums in den Bestand ein. Es entsteht ein dreischichtiger Bestand, dessen Schichten aus mehreren Baumarten bestehen. Auch im Simulationslauf ERTRAG entwickelt sich ein dreischichtiger Bestand. Allerdings besteht er nur aus zwei verschiedenen Baumarten (Kiefer und Douglasie), die sich in der mittleren Schicht mischen. Den nächsthohen Index-A hat der Bestand am Ende der Simulation PROZESS. Auch dieser Bestand gilt als dreischichtig. Die unteren Schichten sind weniger dicht besetzt und bestehen aus fünf Baumarten (Sandbirke, Moorbirke, Eberesche, Fichte und Kiefer). Den geringsten Index-A weist der Bestand nach Ablauf der Simulationsvariante PNV auf. Zwar ist der Bestand ebenfalls dreischichtig, allerdings ist die Mischung der Arten innerhalb der Schichten nicht so vielseitig wie in den drei anderen Varianten. 
Der Bestand erfüllt die Voraussetzung für die Auswahl von Habitatbäumen. Er besteht aus Bäumen, die über der geforderten Mindesthöhe für die Z-Baum-Auswahl liegen. Dennoch wurden in keiner der zwei Varianten (LÖWE und PROZESS), die die Auswahl von Habitatbäumen vorsehen, Habitatbäume in den Beispielläufen ausgewählt, da die Auswahl an eine Zufallskomponente geknüpft ist. Es wird ein Habitatbaum pro Hektar angestrebt, die gewählte Simulationsfläche beträgt aber nur ein Viertel Hektar. Ein Habitatbaum liegt demnach nur mit einer Wahrscheinlichkeit von eins zu vier in der Bestandesfläche. Durch die Ziehung einer Zufallszahl wurde festgelegt, daß weder in der Variante LÖWE noch in der Variante PROZESS ein Habitatbaum ausgewählt wird.

Mit den angestrebten Zieltypen steht das Zielerreichungsprozent zu Beginn der Simulationläufe LÖWE, PNV und ERTRAG fest. Da die Variante PROZESS keinen Zieltyp anstrebt, wird kein Zielerreichungsprozent angegeben.

In den drei Varianten, die Pflanzungen vorsehen (LÖWE, PNV und ERTRAG), werden die zur Zielerreichung notwendigen Pflanzflächen während des Simulationslaufs voll umgesetzt: In der LÖWE-Variante werden Pflanzungen auf $60 \%$, in der PNV-Variante auf $80 \%$ und in der Variante ERTRAG auf $100 \%$ der Bestandesfläche durchgeführt. Dadurch, daß die gepflanzten Bäume bei allen drei Varianten während des Simulationszeitraums einwachsen, die Kiefern aber dennoch einen großen Teil der Kronenschirmfläche im Bestand ausmachen, erhöhen sich in allen drei Varianten die Zielerreichungsprozente. In der Variante PROZESS sind keine Pflanzungen vorgesehen.

\section{Bestand 2}

Die Ergebnisse der Entwicklung des dreißigjährigen Fichtenbestandes (Bestand 2) werden in Tabelle 4.1.1.b aufgeführt. Abbildung 4.1.1.c veranschaulicht das Erscheinungsbild des Bestandes zu Beginn und zum Ende der vier Simulationsvarianten.

Dem dreißigjährigen Fichtenbestand wird in der Variante LÖWE der Zieltyp Fichten/Buchenbestand, in der Variante PNV der Zieltyp Buchenbestand und in der Variante ERTRAG der Zieltyp Fichtenbestand zugeordnet. Im Unterschied zu den Simulationsläufen für den Bestand 1 werden daher nicht nur in der Variante LÖWE, sondern auch in der Variante ERTRAG permanente Z-Bäume ausgewählt. Die Bestände sind zum Simulationsbeginn für Durchforstungsmaßnahmen noch zu jung. In den Varianten LÖWE und PNV findet der erste Eingriff im fünfzehnten, in den Varianten ERTRAG und PROZESS erst im zwanzigsten Simulationsjahr statt. Die Mindesthöhe für die Auswahl von Z-Bäumen liegt in den erstgenannten Varianten bei $14 \mathrm{~m}$, in den letzten beiden bei $16 \mathrm{~m}$. Im Gegensatz zu dem Verlauf der Simulationen des Bestandes 1 werden im zweiten Bestand bis zum Ende des Simulationszeitraums in allen Varianten Eingriffe durchgeführt. Der Bestand 2 erfüllt in keinem der Läufe die Bedingungen zur Durchführung von Pflanzungsmaßnahmen, da er noch zu weit vom Endnutzungszeitpunkt entfernt ist und die Bäume zu dicht stehen. Daher werden auch in den Varianten LÖWE, PNV und ERTRAG, die Pflanzungen vorsehen, keine Pflanzungen durchgeführt.

Die Vornutzungsmasse ist in der Variante PNV erneut höher $(429,8 \mathrm{Vfm} / \mathrm{ha})$ als in den anderen drei Varianten (LÖWE: 298,4, ERTRAG: 290,6, PROZESS: 213,6). Dadurch, daß 


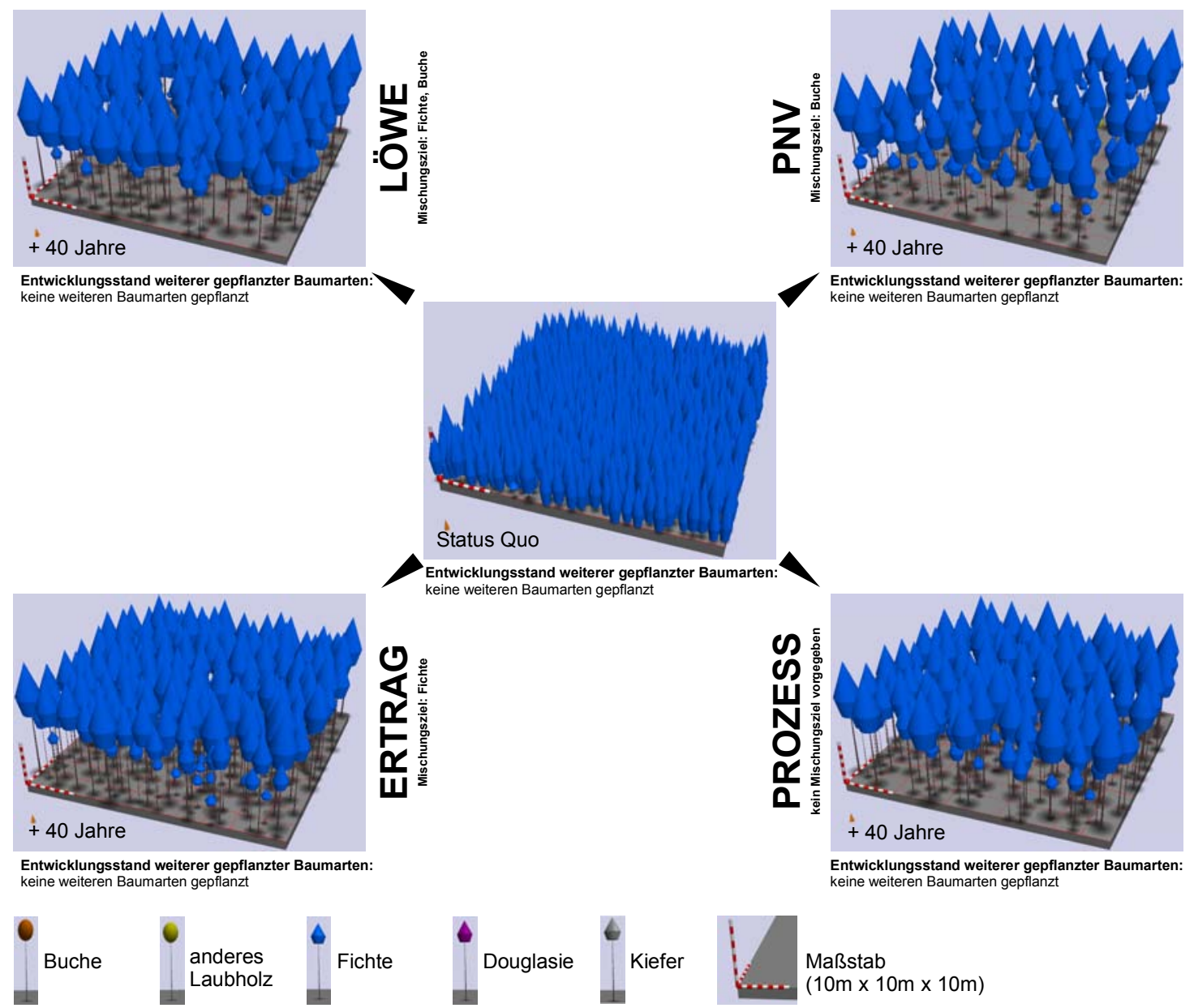

Abbildung 4.1.1.c: Simulationsergebnisse der Szenarien LÖWE, PNV, ERTRAG und PROZESS nach vierzigjähriger Entwicklung eines Bestandes aus dreißigjähriger Fichte (Bestand 2 aus Tabelle 3.2.2.a).

in diesem Bestand, aber auch in den Varianten LÖWE und ERTRAG bis zum Ende der Simulation Eingriffe stattfinden, liegen ihre Vornutzungsmengen deutlich über denen der Variante PROZESS.

Durch die starken Eingriffe differenziert sich der Bestand in der Variante PNV stärker als in den anderen drei Varianten. Gerade in den letzten Jahrzehnten der Simulation wird dadurch der durchschnittliche Durchmesser der ausscheidenen Bäume des Bestandes angehoben. Der höhere Durchmesser wirkt sich auf den durchschnittlichen Wert pro Festmeter (für das entnommene Holz) aus. Er beträgt in dieser Variante 15,50€, in den anderen Varianten liegt er um 13,30€.

Insgesamt führt die hohe Entnahmemenge in der Variante PNV zu einem Gesamterlös aus Vornutzungen von $6672 € /$ ha. Darauf folgt die Variante LÖWE mit $3989 € /$ ha, die Variante ERTRAG mit $3847 € /$ ha und die Variante PROZESS mit $2844 € /$ ha. Auch bei einer Verzinsung der Erträge bleibt dieses Verhältnis unter den Varianten erhalten.

Die Totholzbildungsrate ist in dem dichten Fichtenbestand deutlich höher als in dem zuvor betrachteten Kiefernbestand. Besonders in den Jahren, in denen keine Eingriffe 
stattfinden, fallen viele Bäume durch natürliche Mortalität aus. In den spät eingreifenden Varianten PROZESS und ERTRAG liegt daher die Summe des im Simulationszeitraums anfallenden Totholzvolumens mit 105,2Vfm/ha (in der Variante PROZESS) und $85,6 \mathrm{Vfm} / \mathrm{ha}$ (in der Variante ERTRAG) über den Werten für die Variante LÖWE (78,7Vfm/ha) und PNV (70,3Vfm/ha). In dieser Reihenfolge spiegelt sich neben dem Zeitpunkt des ersten Eingriffs auch die Eingriffsintensität wider. In der Variante ERTRAG werden Bäume stärker als in der Variante PROZESS freigestellt, in der Variante PNV stärker als in der Variante LÖWE.

Der Zuwachs der verschiedenen Varianten unterscheidet sich nur leicht. Die Variante PROZESS liefert einen Zuwachs von 16,5Vfm/ha, die Variante ERTRAG $16 \mathrm{Vfm} / \mathrm{ha}$, die Variante LÖWE 15,9Vfm/ha und die Variante PNV 15,3Vfm/ha. Da keine Pflanzungen stattfinden, beeinflußt Einwuchs aus Pflanzungen die Ergebnisse im Fall des Bestandes 2 im Gegensatz zu Bestand 1 nicht.

Der Derbholzvorrat steigt in allen vier Varianten deutlich an, weil sich der Bestand in einer vorratsaufbauenden Altersstufe befindet. Da sich die Zuwächse nur unwesentlich unterscheiden, steigt der Vorrat umgekehrt proportional zur Vornutzungsmasse. Am höchsten ist er mit $472,7 \mathrm{Vfm} / \mathrm{ha}$ in der Variante PROZESS, danach folgen ERTRAG (396Vfm/ha), LÖWE $(390,2 \mathrm{Vfm} / \mathrm{ha})$ und PNV $(246,2 \mathrm{Vfm} / \mathrm{ha})$.

Im Beispielbestand 2 spielt im Gegensatz zum Beispielbestand 1 Einwuchs aus Pflanzung keine Rolle. Daher sind die erntekostenfreien Abtriebswerte eng mit der Masse und der Struktur des Derbholzvorrats verknüpft. Die Variante PROZESS kommt mit $8776 € /$ ha auf den höchsten erntekostenfreien Abtriebswert. Den geringsten Wert hat der Bestand am Ende der Simulation der Variante PNV mit 4913€/ha. Obwohl die Varianten LÖWE und ERTRAG einen ähnlich hohen Derbholzvorrat am Ende der Simulation vorweisen, liegt der erntekostenfreien Abtriebswert der Variante LÖWE (7771€/ha) über dem der Variante ERTRAG (7262€/ha). Der Wert des verbliebenen Bestandes liegt mit 19,90€/ha in der Variante LÖWE ca. 1,60€/ha höher als in der Variante ERTRAG. Der Bestand hat sich unter den Durchforstungeingriffen in der Variante LÖWE besser differenziert, und die stärkeren Einzelbäume führen unter der angenommenen Preisentwicklung zu einem höheren Gesamtwert.

Die Spanne zwischen dem erntekostenfreien Abtriebswert zu Beginn und zum Ende der Simulation ist im Beispielbestand 2 wesentlich höher als im Beispielbestand 1. Das und die fehlenden Pflanzungskosten führen zu den durchweg positiven Holzproduktionswerten der Varianten. Trotz des niedrigsten erntekostenfreien Abtriebswerts der Variante PNV ist ihr Holzproduktionswert mit 240€/ha/a am höchsten. Der Grund liegt in den hohen Erlösen aus den Vornutzungen, die beim Anderthalb- bis Zweifachen der anderen Varianten liegen. Die Variante LÖWE liegt mit einem Holzproduktionswert von $237 € /$ ha/a nur knapp unter dem Wert der Variante PNV. Im Anbetracht der Tatsache, daß sie einen wesentlich höheren Abtriebswert am Ende des Simulationszeitraums vorweist, ist dieses Ergebnis besonders positiv zu bewerten. Daß die Variante ERTRAG mit 223€/ha/a noch hinter der Variante PROZESS $(225 € /$ ha/a) abschneidet, liegt an ihrem höheren erntekostenfreien Abtriebswert. 
Der Index-A steigt vom Ausgangswert 0 auf einen Wert, der um 0,5 liegt. Die Unterschiede zwischen den Varianten PROZESS $(0,60)$, ERTRAG $(0,58)$, LÖWE $(0,56)$ und PNV $(0,50)$ können durch Zufallseffekte erklärt werden, die beim Einwuchs aus Naturverjüngung eine Rolle spielen und Einfluß auf die Art der eingewachsenen Bäume haben.

Zur Auswahl von Habitatbäumen kommt es auch bei der Simulation des Bestandes 2 zufallsbedingt nicht.

Das Zielerreichungsprozent ändert sich über den Simulationszeitraum nicht. Die wenigen Bäume aus Einwuchs, die auf Naturverjüngung zurückzuführen sind, haben keine wesentlichen Auswirkungen auf die dominierende Stellung der Fichte bei der Betrachtung der Kronenschirmflächenverhältnisse im Bestand. Wegen der Bestandesdichte werden keine neuen Arten gepflanzt, und somit findet auch kein Einwuchs aus Pflanzung statt, der die Kronenschirmflächenverhältnisse beeinflussen könnte.

Tabelle 4.1.1.b: Übersicht über die Ergebnisse der Simulation des Bestandes 2 anhand der Auswertungsparameter.

\begin{tabular}{|c|c|c|c|c|c|c|}
\hline Kriterium & Parameter & Status Quo & $L \ddot{O} W E$ & $P N V$ & ERTRAG & PROZESS \\
\hline \multirow[t]{2}{*}{$\begin{array}{l}\text { Forstliche } \\
\text { Ressourcen }\end{array}$} & $\begin{array}{l}\text { Derbholzvorrat } \\
(\mathrm{Vfm} / \mathrm{ha})\end{array}$ & 131,2 & 390,2 & 246,2 & 396,0 & 472,7 \\
\hline & Abtriebswert (E/ha) & -652 & 7771 & 4913 & 7262 & 8776 \\
\hline \multirow[t]{7}{*}{$\begin{array}{l}\text { Produktions- } \\
\text { funktion }\end{array}$} & $\begin{array}{l}\text { Vornutzungsmasse } \\
\text { (Vfm/ha) }\end{array}$ & keine Angabe & 298,4 & 429,8 & 290,6 & 213,6 \\
\hline & $\begin{array}{l}\text { Endnutzungsmasse } \\
\text { (Vfm/ha) }\end{array}$ & keine Angabe & 0 & 0 & 0 & 0 \\
\hline & $\begin{array}{l}\text { Zuwachs } \\
(\text { Vfm/ha/a) }\end{array}$ & keine Angabe & 15,9 & 15,3 & 16,0 & 16,5 \\
\hline & $\begin{array}{l}\text { erntekostenfreie } \\
\text { Holzerlöse } \\
\text { Vornutzungen } \\
(€ / \text { ha })\end{array}$ & keine Angabe & 3989 & 6672 & 3847 & 2844 \\
\hline & $\begin{array}{l}\text { erntekostenfreie } \\
\text { Holzerlöse } \\
\text { Endnutzungen } \\
(€ / \text { ha })\end{array}$ & keine Angabe & 0 & 0 & 0 & 0 \\
\hline & $\begin{array}{l}\text { Pflanzfläche } \\
\text { (\% der Bestandesfläche) }\end{array}$ & keine Angabe & 0 & 0 & 0 & 0 \\
\hline & $\begin{array}{l}\text { Holzproduktionswert } \\
(\epsilon / h a / a)\end{array}$ & keine Angabe & 237 & 240 & 223 & 225 \\
\hline \multirow{3}{*}{$\begin{array}{l}\text { Biologische } \\
\text { Diversität }\end{array}$} & Index- $A$ & 0 & 0,56 & 0,50 & 0,58 & 0,60 \\
\hline & $\begin{array}{l}\text { Totholzvolumen } \\
\text { (Vfm/ha) }\end{array}$ & keine Angabe & 78,7 & 70,3 & 85,6 & 105,2 \\
\hline & $\begin{array}{l}\text { Habitatbaumvolumen } \\
(\mathrm{Vfm} / \mathrm{ha})\end{array}$ & keine Angabe & 0 & 0 & 0 & 0 \\
\hline Zielerreichung & $\begin{array}{l}\text { Zielerreichungsprozent } \\
\text { (\%) }\end{array}$ & $\begin{array}{l}\text { LÖWE: } 70 \\
\text { PNV:10 } \\
\text { ERTRAG: } 100 \\
\text { PROZESS: } x\end{array}$ & 70 & 10 & 100 & $x$ \\
\hline
\end{tabular}




\section{Bestand 3}

In Abbildung 4.1.1.d und Tabelle 4.1.1.c wird das Ergebnis der Fortschreibung des dritten Beispielbestandes, einem Mischbestand aus fünfzigjährigen Kiefern und dreißigjährigen Fichten, vorgestellt. Wie die beiden vorherigen Beispiele finden auch in den vier Simulationsläufen des Bestandes 3 nur Vornutzungen und keine Endnutzungen statt. In der Variante LÖWE wird ein Fichten-/ Buchenmischbestand, in der Variante PNV dagegen ein Buchenbestand und in der Variante ERTRAG ein Fichtenbestand angestrebt.

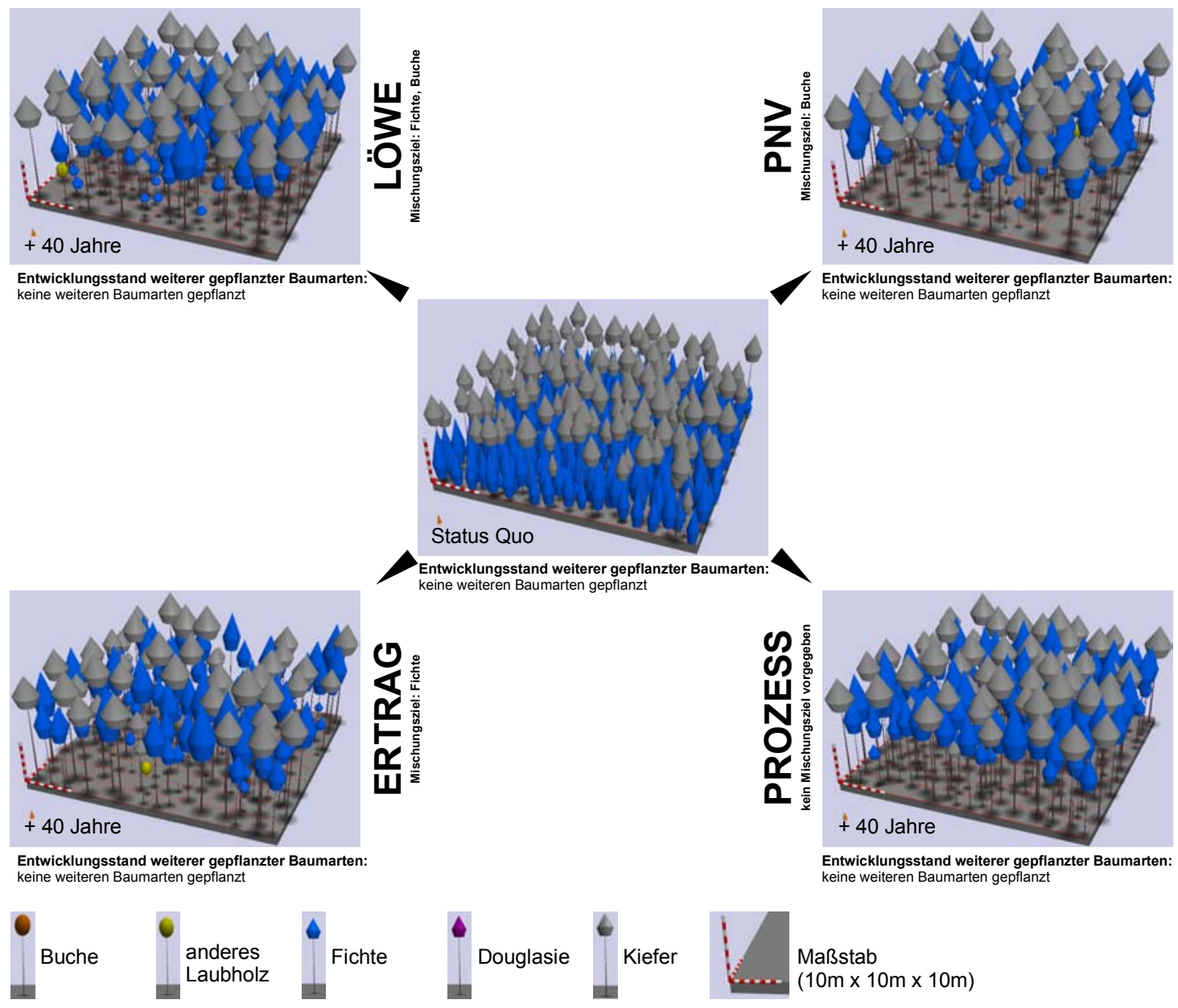

Abbildung 4.1.1.d: Simulationsergebnisse der Szenarien LÖWE, PNV, ERTRAG und PROZESS nach vierzigjähriger Entwicklung eines Bestandes aus fünfzigjähriger Kiefer und dreißigjähriger Fichte (Bestand 3 aus Tabelle 3.2.2.a).

Die Durchforstungseingriffe beschränken sich in allen vier Varianten so lange auf die Kiefern des Bestandes, bis die Fichten die für die Durchforstung geforderte Mindesthöhe erreichen. In den Varianten LÖWE und PNV wird im fünfzehnten, bei den Varianten ERTRAG und PROZESS erst im dreißigsten Jahr in die Fichte eingegriffen. Die gesamte Vornutzungsmasse akkumuliert sich über den Simulationszeitraum in der Variante PNV auf $374,8 \mathrm{Vfm} / \mathrm{ha}$, in der Variante ERTRAG auf $335,1 \mathrm{Vfm} / \mathrm{ha}$, in der Variante LÖWE auf $260,6 \mathrm{Vfm} / \mathrm{ha}$ und in der Variante PROZESS auf $208,4 \mathrm{Vfm} / \mathrm{ha}$. Wieder wird in der Variante PNV am stärksten eingegriffen, da dies den vollständigen Umbau des Kiefern- 
/Fichtenbestandes in einen Buchenbestand unterstützt. Die Werte aller Varianten liegen über den Massen, die im reinen Kiefernbestand und unter den Massen, die im reinen Fichtenbestand entnommen wurden.

Tabelle 4.1.1.c: Übersicht über die Ergebnisse der Simulation des Bestandes 3 anhand der Auswertungsparameter.

\begin{tabular}{|c|c|c|c|c|c|c|}
\hline Kriterium & Parameter & Status Quo & $L \ddot{O} W E$ & PNV & ERTRAG & PROZESS \\
\hline \multirow[t]{2}{*}{$\begin{array}{l}\text { Forstliche } \\
\text { Ressourcen }\end{array}$} & $\begin{array}{l}\text { Derbholzvorrat } \\
\text { (Vfm/ha) }\end{array}$ & 192,1 & 395,1 & 287,4 & 311,0 & 423,4 \\
\hline & Abtriebswert (E/ha) & 172 & 6370 & 4887 & 5163 & 7067 \\
\hline \multirow[t]{7}{*}{$\begin{array}{l}\text { Produktions- } \\
\text { funktion }\end{array}$} & $\begin{array}{l}\text { Vornutzungsmasse } \\
\text { (Vfm/ha) }\end{array}$ & keine Angabe & 260,6 & 374,8 & 335,1 & 208,4 \\
\hline & $\begin{array}{l}\text { Endnutzungsmasse } \\
\text { (Vfm/ha) }\end{array}$ & keine Angabe & 0 & 0 & 0 & 0 \\
\hline & $\begin{array}{l}\text { Zuwachs } \\
(\text { Vfm/ha/a) }\end{array}$ & keine Angabe & 13,2 & 13,0 & 13,3 & 14,0 \\
\hline & $\begin{array}{l}\text { erntekostenfreie } \\
\text { Holzerlöse Vornutzungen } \\
\text { (E/ha) }\end{array}$ & keine Angabe & 4165 & 6674 & 5552 & 3194 \\
\hline & $\begin{array}{l}\text { erntekostenfreie } \\
\text { Holzerlöse } \\
\text { Endnutzungen } \\
(\text { E/ha })\end{array}$ & keine Angabe & 0 & 0 & 0 & 0 \\
\hline & $\begin{array}{l}\text { Pflanzfläche } \\
\text { (\% der Bestandesfläche) }\end{array}$ & keine Angabe & 0 & 0 & 0 & 0 \\
\hline & $\begin{array}{l}\text { Holzproduktionswert } \\
\text { (E/ha/a) }\end{array}$ & keine Angabe & 168 & 185 & 171 & 164 \\
\hline Biologische & Index $-A$ & 1,10 & 0,89 & 0,78 & 0,82 & 0,87 \\
\hline \multirow[t]{2}{*}{ Diversität } & $\begin{array}{l}\text { Totholzvolumen } \\
\text { (Vfm/ha) }\end{array}$ & keine Angabe & 65,5 & 49,1 & 79,2 & 118,8 \\
\hline & $\begin{array}{l}\text { Habitatbaumvolumen } \\
\text { (Vfm/ha) }\end{array}$ & keine Angabe & 0 & 0 & 0 & 0 \\
\hline Zielerreichung & $\begin{array}{l}\text { Zielerreichungsprozent } \\
\text { (\%) }\end{array}$ & $\begin{array}{l}\text { LÖWE: } 40 \\
\text { PNV: } 10 \\
\text { ERTRAG: } 50 \\
\text { PROZESS: } \mathrm{x}\end{array}$ & 40 & 10 & 50 & $x$ \\
\hline
\end{tabular}

Die erntekostenfreien Holzerlöse sind, wie in den Simulationsläufen der Bestände 1 und 2, auch hier in der Variante PNV (mit 4931€/ha) am höchsten. Es folgen die Werte der Variante ERTRAG (4401€/ha), der Variante LÖWE (3209€/ha) und der Variante PROZESS (2617€/ha).

Wie in den Simulationsläufen zu den Beständen 1 und 2 fällt auch in Bestand 3 mit $118,8 \mathrm{Vfm} /$ ha die höchste Totholzmenge in der Variante PROZESS an. Die über den Simulationszeitraum akkumulierten Werte betragen in der Variante ERTRAG 79,2Vfm/ha, in der Variante LÖWE 65,5Vfm/ha und in der Variante PNV 49,1Vfm/ha.

In den Zuwachs fließt wie in Bestand 2 auch in den Simulationsläufen des Bestandes 3 kein Einwuchs aus Pflanzungen ein. Daher unterscheiden sich die Werte im Vergleich zu den Werten, die für Bestand 1 errechnet werden, nur leicht. Der Zuwachs in Bestand 3 liegt in der Variante PROZESS mit $14 \mathrm{Vfm} / \mathrm{ha}$ am höchsten, gefolgt von den Varianten ERTRAG mit 13,3Vfm/ha und LÖWE mit 13,2Vfm/ha. Die Variante PNV liefert mit 13,0 Vfm/ha den niedrigsten Wert. 
Wie in Bestand 2 liegt der Derbholzvorrat der Variante PROZESS mit 423,4Vfm/ha zum Abschluß der Simulation am höchsten. Danach folgen die Werte von LÖWE (mit $395,1 \mathrm{Vfm} / \mathrm{ha}$ ) und ERTRAG (mit 311Vfm/ha). Wie in den Beispielbeständen 1 und 2 senken die starken Eingriffe die Masse in der Variante PNV so stark herab, daß auch im Mischbestand der Derbholzvorrat bei dieser Variante mit $287,4 \mathrm{Vfm} /$ ha deutlich unter dem der anderen drei Varianten liegt.

Auf den erntekostenfreien Abtriebswert haben die wenigen Individuen, die während des Simulationszeitraums eingewachsen sind, kaum einen nennenswerten Einfluß. Die Werte pro Festmeter differieren unter den Varianten weniger als in den beiden vorherigen Beispielen. Der Grund dafür liegt in der Struktur des Bestandes. Dadurch, daß er aus zwei unterschiedlich alten und unterschiedlich hohen Baumarten besteht, ist der Konkurrenzdruck der Einzelbäume innerhalb der Schichten insgesamt geringer, wodurch der Effekt der Durchforstungsstrategie nicht mehr so stark zu erkennen ist. Der durchschnittliche Wert der verbleibenden Bäume liegt bei allen vier Varianten um 16,5€ pro Festmeter. Bedingt durch den hohen Derbholzvorrat ist der Abtriebswert des Bestandes der Variante PROZESS nach dem vierzigjährigen Simulationszeitraum mit $7067 € /$ ha am höchsten, gefolgt von LÖWE mit $6370 € /$ ha und ERTRAG mit 5163€/ha. Am niedrigsten ist der Wert des Bestandes nach der Fortschreibung mit $4887 € /$ ha in der Variante PNV - dieser Bestand besitzt auch den geringsten Derbholzvorrat. Der Holzproduktionswert ist in allen vier Varianten positiv, er liegt in der Variante PNV bei $185 € / \mathrm{ha} / \mathrm{a}$, in der Variante ERTRAG bei $171 € / \mathrm{ha} / \mathrm{a}$, in der Variante LÖWE bei $168 € / \mathrm{ha} / \mathrm{a}$ und in der Variante PROZESS bei 164€/ha/a. In diesem Bestand sind die Werte besonders durch die erntekostenfreien Holzerlöse aus den Vornutzungen geprägt. Zum Beispiel kann der hohe Abtriebswert des Bestandes am Ende der Simulation der Variante PROZESS die geringen Einnahmen aus den Vornutzungen nicht ausgleichen. Die Variante PNV verdankt ihr gutes Abschneiden hauptsächlich ihren hohen Einnahmen aus den Vornutzungen.

Im Status Quo hat der Bestand 3 einen Index-A von 1,10. Nach dem Simulationszeitraum ist dieser Wert in der Variante LÖWE auf 0,89, in der Variante PROZESS auf 0,87, in der Variante ERTRAG auf 0,82 und in der Variante PNV auf 0,78 gefallen. Die Unterschiede zwischen den Varianten basieren, wie in Bestand 2, größtenteils auf der zufälligen Auswahl der einwachsenden Baumarten. Wird zum Beispiel zwei einwachsenden Bäumen die gleiche Baumart zugeordnet, so ist die Diversität geringer als wenn sie unterschiedliche Arten besäßen.

Auch in den Simulationsläufen für den Bestand 3 kommt es zufallsbedingt nicht zu einer Auswahl von Habitatbäumen.

Die in den Varianten LÖWE, PNV und ERTRAG vorgesehenen Pflanzungen werden wegen der Bestandesdichte nicht während des Simulationszeitraums durchgeführt. Die Zielerreichungsprozente ändern sich daher in keiner der Varianten. 


\subsubsection{Beispiel Endnutzung}

Anhand des Bestandes 4 (siehe Tabelle 3.2.2.a) sollen die unterschiedlichen Endnutzungsroutinen der Varianten LÖWE, PNV, ERTRAG und PROZESS verdeutlicht werden.

Der Ausgangsbestand besteht aus hundertzehnjähriger Kiefer, die mit fünfzehnjähriger Buche unterbaut ist. Eine solche Konstellation ist ein typisches Umbaubeispiel aus dem Untersuchungsgebiet. In der Variante LÖWE wird ein Kiefern-/ Douglasien-/ Buchenmischbestand, in der Variante PNV ein reiner Buchenbestand und in der Variante ERTRAG ein reiner Douglasienbestand angestrebt. Da der Bestand jedoch mit Buche unterbaut ist, kommt es während des Simulationszeitraums zu keinen weiteren Pflanzungen.

Der Umbau des Bestandes kann erst angegangen werden, wenn die Buchen in das Endnutzungsalter kommen. Innerhalb des Simulationszeitraums wird diese Phase jedoch nicht mehr abgebildet.

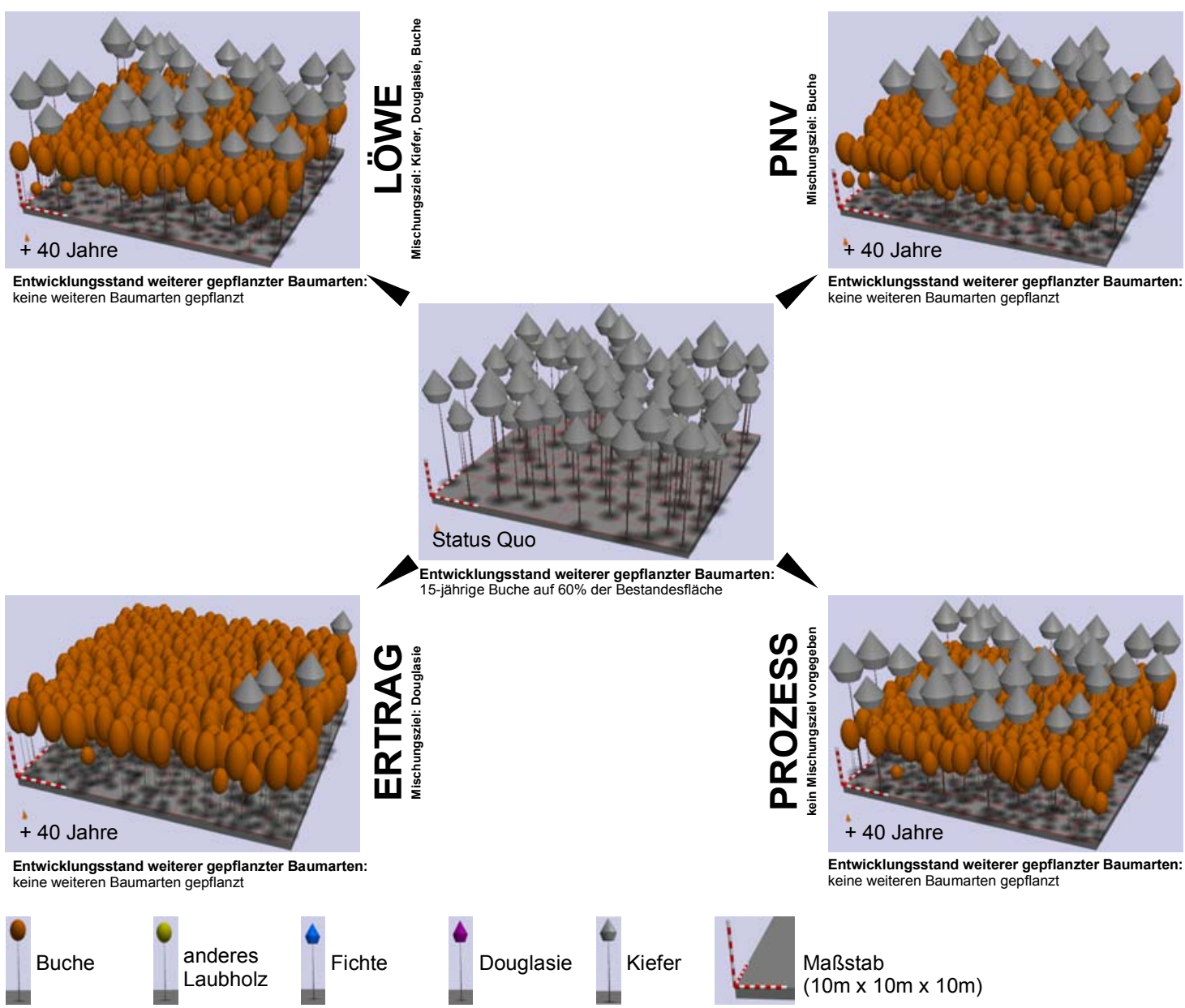

Abbildung 4.1.2.a: Simulationsergebnisse der Szenarien LÖWE, PNV, ERTRAG und PROZESS nach vierzigjähriger Entwicklung eines Bestandes aus hundertzehnjähriger Kiefer (Bestand 4 aus Tabelle 3.2.2.a). 
Abbildung 4.1.2.a zeigt den Bestand im Status Quo und in den vier Zuständen, die sich nach dem Ablauf des Simulationszeitraums bei den vier verschiedenen Simulationsvarianten ergeben. Wie es zu den unterschiedlichen Ergebnissen kommt, kann den in Abbildung 4.1.2.b dargestellten Stammverteilungsplänen entnommen werden. Sie zeigen den Verlauf der Simulationen in zehn Jahresschritten.

Zunächst sollen laut den Maßnahmenketten der Varianten LÖWE und PROZESS Habitatbäume ausgewählt werden. Die zufallsgesteuerte Auswahlroutine führt jedoch nur im Simulationslauf LÖWE zur Markierung eines Habitatbaums. Dieser Habitatbaum wird auch nachdem er zielstark geworden ist, nicht aus dem Bestand entfernt.

In den Varianten LÖWE, PNV und ERTRAG ist die Auswahl permanenter Z-Bäume Teil ihrer Maßnahmenketten (siehe Kapitel 3.5.1 bis 3.5.4). Zu einer Markierung permanenter Z-Bäume kommt es allerdings nur in der Variante LÖWE. Wie im Zusammenhang mit der Entwicklung des Bestandes 1 beschrieben, ist dies darauf zurückzuführen, daß nur bei der Variante LÖWE Kiefern als Teil ihres Zieltyps vorgesehen sind. Mit der Freistellung der permanenten Z-Bäume ist in der Variante LÖWE die maximale Entnahmemasse für Vornutzungseingriffe erreicht. Die im weiteren Verlauf markierten temporären Z-Bäume werden daher nicht weiter gefördert.

Die Auswahl der temporären Z-Bäume führt in den Varianten PNV, ERTRAG und PROZESS zu identischen Ergebnissen. Die Anzahl der tatsächlich freizustellenden ZBäume ist deshalb reduziert, weil ein Z-Baum, der $90 \%$ seines Zieldurchmessers erreicht hat, nicht mehr freigestellt wird. Nur in der Variante PNV kommt es zu Simulationsbeginn (Jahr 0) und im darauffolgenden Eingriffsjahr (Jahr 5 - ohne Abbildung) zu Durchforstungseingriffen, da nur in dieser Variante die Mindestentnahmemenge erreicht werden kann. Die Ursachen dafür sind die im Vergleich zur Variante ERTRAG hohen Zielstärken, niedrigeren Mindestentnahmemenge und der im Vergleich zur Variante PROZESS hohe Freistellungsgrad der Variante PNV.

Bis zum 20. Simulationsjahr finden in der Simulation nach dem LÖWE-Konzept keine weiteren Eingriffe in den Bestand statt. Im 20. Simulationsjahr beginnt dann die Endnutzung, da dann eine ausreichende Anzahl an Kiefern ihre Zielstärke erreicht hat, so daß die Mindestmasse für Endnutzungen überschritten werden kann. Es erreichen sogar so viele Kiefern zum gleichen Zeitpunkt ihre Zielstärke, daß die Entnahmemenge von der maximal zugelassenen Endnutzungsmasse begrenzt wird und einige zielstarke Bäume bis zum nächsten Eingriff in Bestand verbleiben. Auch im 25. und im 35. Simulationsjahr können die Mindestmengen erreicht werden und Endnutzungsmaßnahmen finden statt. Im 40. Jahr der Simulation haben einige Buchen die zur Markierung von Z-Bäumen geforderte Mindesthöhe erreicht und werden daraufhin als permanente Z-Bäume gekennzeichnet und bevorzugt freigestellt. Die Zielstärke und die maximalen und minimalen Endnutzungsmassen der Varianten LÖWE, PNV und PROZESS sind identisch. Da lediglich Unterschiede der Varianten in den Durchforstungsregeln zu finden sind und nur in den Fällen LÖWE und PNV ein Durchforstungseingriff zu Beginn der Simulation stattfindet, sind die Unterschiede bei der Endnutzung hauptsächlich durch zufällige Wachstumseffekte zu erklären. 


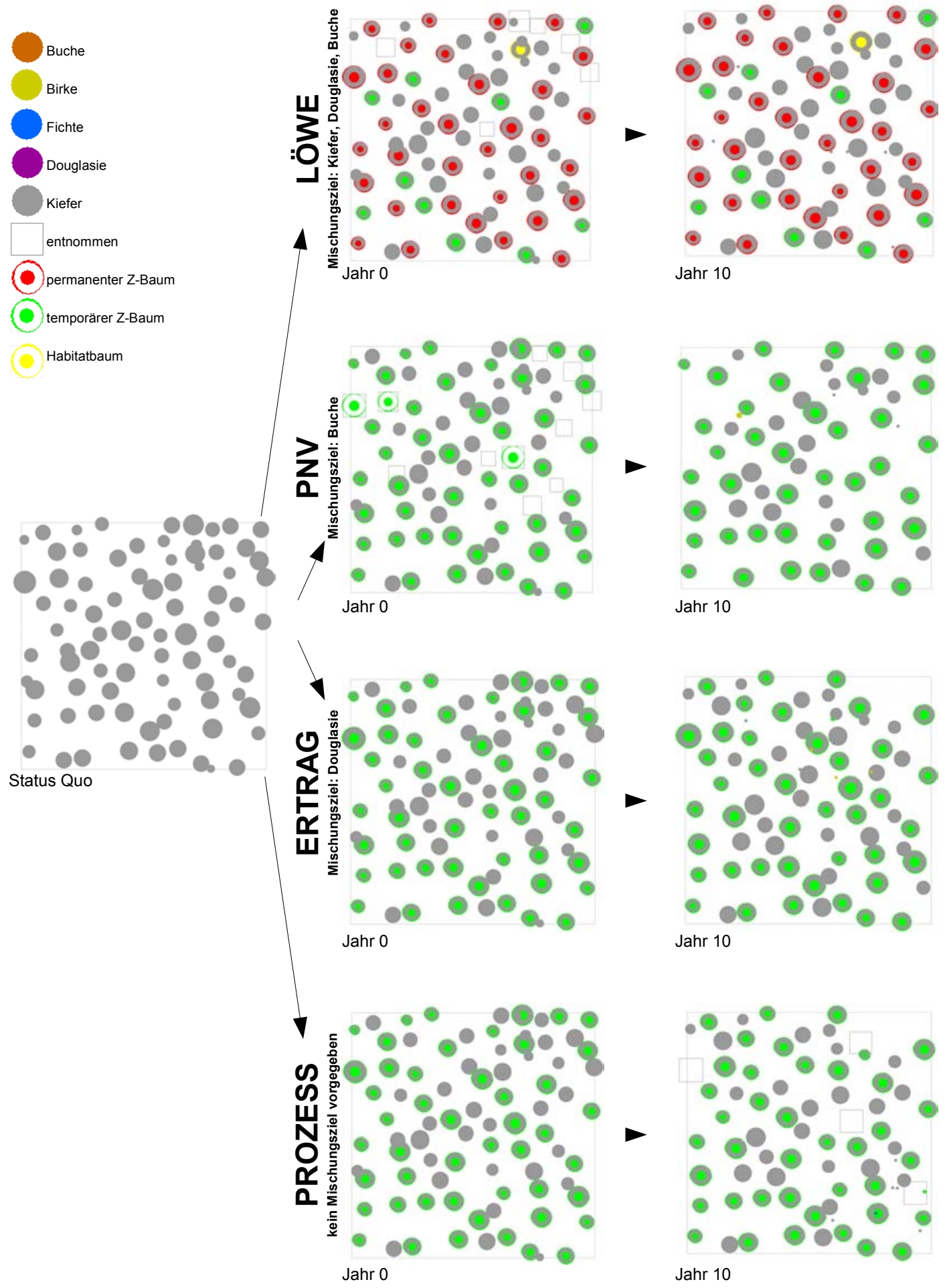

Abbildung 4.1.2.b: Die vierzigjährige Entwicklung eines Bestandes aus Stammverteilungsplänen des Status-Quo und der Zwischenergebnisse der Szenarien Jahren festgehalten worden (auch für die Strategien LÖWE und PNV, die zwei Grundfläche dargestellt. 

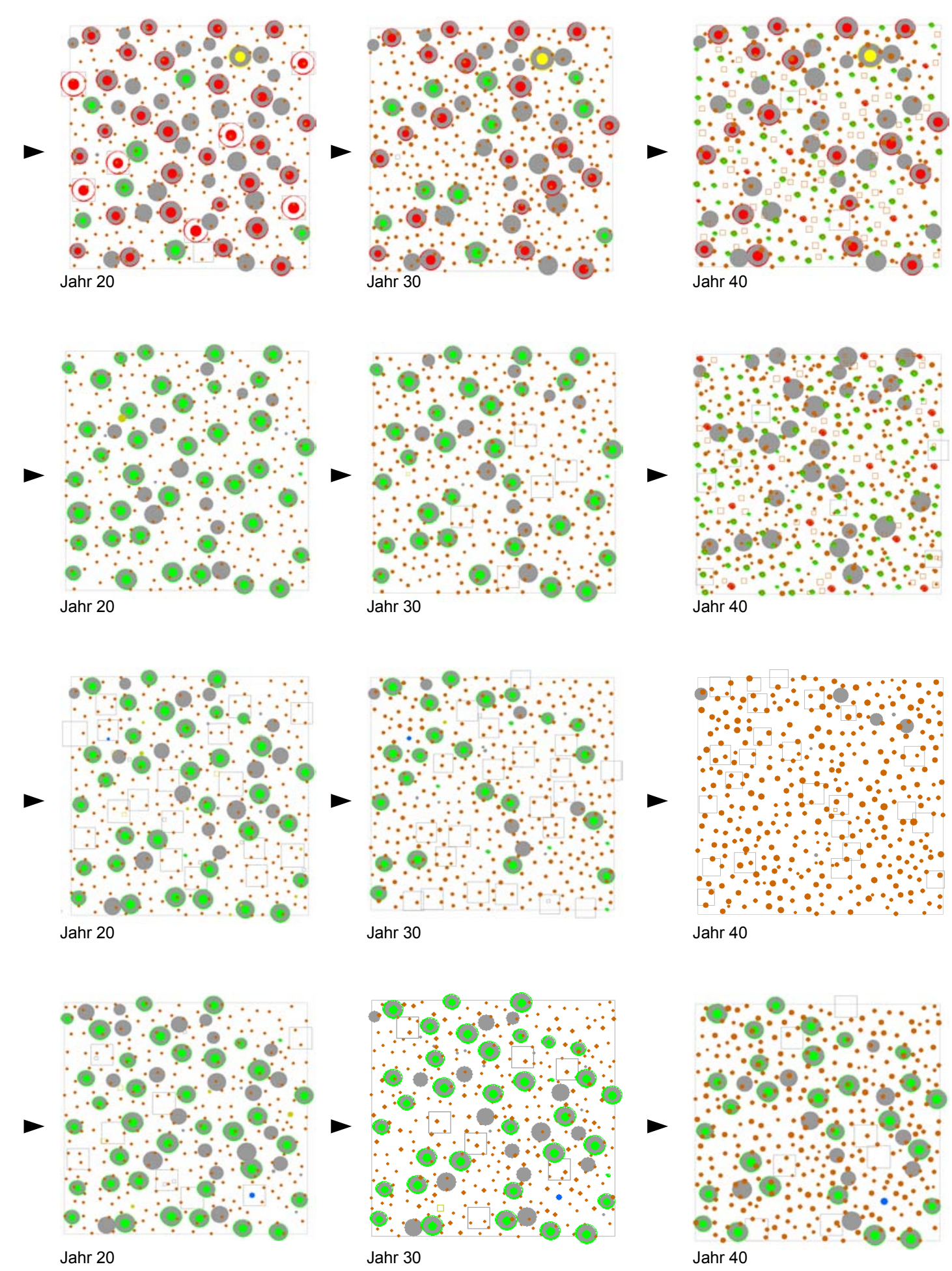

hundertzehnjähriger Kiefer (Bestand 3 aus Tabelle 3.2.2.a) anhand von LÖWE, PNV, ERTRAG und PROZESS. Die Einzelbilder sind im Abstand von zehn Eingriffe im Jahrzehnt vorsehen). Die Stämme werden mit einer dreifach vergrößerten 
Endnutzungseingriffe finden in der Variante PNV in den Jahren 15, 20, 25, 30, 35 und 40 nach Simulationsbeginn statt. In der Variante PROZESS werden Bäume im Abstand von zehn Jahren ab dem zehnten Simulationsjahr im Rahmen einer Endnutzung entnommen. In den Eingriffen werden im Vergleich zu den Varianten mit einem fünfjährigen Eingriffsintervall ungefähr doppelt so viele Bäume genutzt. Daher ähneln sich die Bilder der Bestände im vierzigsten Simulationjahr stark.

Von diesen drei Varianten unterscheidet sich die Variante ERTRAG grundsätzlich. Der erste Endnutzungseingriff wird im Jahr 20 der Simulation durchgeführt. In diesem Jahr hat die führende Bestandesschicht aus Kiefern den als Zielstärke geforderten Dg von $40 \mathrm{~cm}$ BHD erreicht. Da im Vergleich $z u$ den anderen drei Varianten eine relativ hohe Endnutzungsmasse zugelassen ist, fällt dieser Eingriff stärker als in den anderen drei Varianten aus. Ab diesem ersten Endnutzungseingriff sieht die Variante ERTRAG die Endnutzung der führenden Schicht innerhalb des für Kiefernbestände vorgesehenen Zeitraums von 20 Jahren vor. Im 30. Simulationsjahr findet daher wiederum ein starker Endnutzungshieb statt, und im 40. Jahr der Simulation werden alle bis dahin verbliebenen Kiefern der Bestandesschicht 1 entfernt. Zurück bleibt, im Gegensatz zu den anderen drei Varianten, ein einschichtiger Bestand aus Buchen, in dem einige vormals unterständige Kiefern verblieben sind.

Wie schon anhand der Stammverteilungspläne beschrieben wurde, unterscheiden sich die Varianten LÖWE, PNV und PROZESS lediglich zu Beginn darin, daß noch Vornutzungen in den Varianten LÖWE und PNV durchgeführt werden. Der Tabelle 4.1.2.a können die Auswirkungen der verschiedenen Endnutzungskonzepte auf die Auswertungsparameter entnommen werden. Bei der Endnutzung fällt über den Simulationszeitraum gesehen in der Variante PROZESS ungefähr die doppelte Menge an zielstarken Bäumen im Vergleich zu den Varianten LÖWE und PNV an. Da die maximale Entnahmemenge damit noch nicht überschritten wird, kommt die Variante in der Summe auf eine ähnliche Endnutzungsmasse wie die Varianten LÖWE und PNV. Am geringsten fällt die Endnutzungsmasse in der Variante LÖWE mit 184,0Vfm/ha in den vierzig Jahren aus. Darauf folgen die Varianten PNV mit 198,1Vfm/ha und die Variante PROZESS mit 233,55Vfm/ha. Die Endnutzungsmenge der Variante ERTRAG liegt durch das grundlegend abweichende Endnutzungskonzept mit 445,8Vfm/ha ungefähr doppelt so hoch.

Auch die erntekostenfreien Holzerlöse liegen in dieser Variante mit 10769€/ha grob beim Zweifachen der anderen Werte. Da jedoch viele Bäume in der Variante ERTRAG mit geringem Durchmesser entnommen werden, liefern sie im Schnitt einen schlechteren Ertrag pro Festmeter $(24,10 € / \mathrm{Vfm})$ als die anderen drei Varianten (dort liegen sie zwischen 25 und $26 € / \mathrm{Vfm})$. Die Bäume verbleiben in den anderen Varianten wegen der höheren Zielstärke länger im Bestand, so daß sie bei ihrer späteren Entnahme höhere Erlöse erzielen. In der Variante PROZESS kommt man insgesamt auf $6081 € /$ ha, in der Variante PNV auf 5079€/ha und in der Variante LÖWE auf 4639€/ha für die erntekostenfreien Holzerlöse aus Endnutzung. Die Variante PROZESS hat höhere Endnutzungsmassen und auch höhere Erlöse aus der Endnutzung, da in dieser Variante früher mit der Endnutzung begonnen wird. Dieser Effekt ist rein zufallsbedingt. Die geforderte minimale Endnutzungsmasse wird zu einem früheren Zeitpunkt als bei den anderen beiden 
Varianten erreicht. Zum Gesamtergebnis der Varianten LÖWE und PNV tragen auch noch die Vornutzungen bei. Die Summe der erntekostenfreien Holzerlöse aus Vor- und Endnutzungen übertreffen das Gesamtergebnis der Variante ERTRAG jedoch nicht. Die Summe beträgt für die Variante LÖWE 5550€/ha und für die Variante PNV durch ihre starken Vornutzungsmaßnahmen 7870€/ha.

Durch die vollständige Entnahme der führenden Schicht aus Kiefern wird der Derbholzvorrat bei der Variante ERTRAG von $378,8 \mathrm{Vfm} /$ ha auf $124,8 \mathrm{Vfm} /$ ha gesenkt. Der erntekostenfreie Abtriebswert des verbliebenen Buchenbestandes beträgt gerade einmal $39 € /$ ha. Die Unterschiede bei den Derbholzvorräten in den Endbeständen der Varianten LÖWE $(345,6 \mathrm{Vfm} / \mathrm{ha})$, PROZESS $(331,0 \mathrm{Vfm} / \mathrm{ha})$ und PNV $(264,1 \mathrm{Vfm} / \mathrm{ha})$ sind auf die durchgeführten Vornutzungen und durch Zufallseffekte zu erklären. Die erntekostenfreien Abtriebswerte belaufen sich auf 5931€/ha in der Variante LÖWE, 5144€/ha in der Variante PROZESS und $3669 € /$ ha in der Variante PNV.

Der Holzproduktionswert ist in allen vier Varianten negativ. Am niedrigsten liegt er bei der Variante PROZESS mit $-58 €$, dann folgen ERTRAG mit -54€, LÖWE mit -50€ und PNV mit $-18 €$. Wird der negative Abtriebswert der Buchen zum Ende des Simulationszeitraums aus dem Holzproduktionswert herausgerechnet, so korrigieren sich die Beträge um 2-3 Euro nach oben. Berechnet man den Holzproduktionswert ab dem Zeitpunkt der ersten Endnutzungsmaßnahme bis vierzig Jahre nach diesem Zeitpunkt, so bewegt sich der Holzproduktionswert zwischen $-2 €$ in der Variante PROZESS und $2 €$ für die Variante PNV. Bei den in dieser Arbeit unterstellten Erlösen für Kiefer (siehe Abbildungen 3.6.2.a und 3.6.2.b) und einem Realzins von $2 \%$ wirft demnach ein endnutzungsreifer Kiefernbestand bei allen vier Varianten nur schwache Überschüsse ab oder muß als negative Investition gesehen werden.

Obwohl in der Variante PROZESS ab dem zehnten Simulationsjahr Endnutzungserlöse anfallen und der Bestand am Ende der Simulationsperiode den zweithöchsten Abtriebswert besitzt, ist der Holzproduktionswert in dieser Variante am niedrigsten. Die beiden anderen zielstärkennutzenden Varianten LÖWE und PNV schneiden besser ab. Es gelingt ihnen, durch die Durchforstungseingriffe die Wertentwicklung des Bestandes positiv zu beeinflussen und sie profitieren von den Erlösen aus den Vornutzungen. Die am besten abschneidende Variante PNV verbindet die gewinnbringenden Durchforstungseingriffe mit einer Zielstärkennutzung, die bei der Diskontierung der Erlöse von den fünfjährigen Eingriffsintervallen profitiert.

Durch die niedrigere Zielstärke, die in der Variante ERTRAG angestrebt wird, entgehen dem Nutzer Erträge, die durch die Wertsteigerung des Holzes zu erwarten gewesen wären. Im Gegenzug wird das frühere Anfallen der Endnutzungserträge im Holzproduktionswert mit der Diskontierung berücksichtigt. Die prozentuale Wertsteigerung des Holzes ist jedoch höher als der angenommene Realzins, weshalb eine spätere Nutzung finanziell vorteilhafter ausfallen würde. Bei der Betrachtung der Variante ERTRAG sind jedoch noch weitere Faktoren zu beachten, die nicht in der Simulation berücksichtigt werden. Erstens wird die Buche durch die frühe Freistellung begünstigt. Ihr Dg liegt ca. $1,5 \mathrm{~cm}$ über denen, die sie in den anderen Varianten bis zum Ende der Simulation erreicht. Die Verkürzung der Umtriebszeit der Buche wird aber im 
Holzproduktionswert, der sich nur auf die Simulationsperiode von 40 Jahren bezieht, nicht berücksichtigt. Zweitens sind Ernteschäden nicht Gegenstand der Simulation. Bei der Entnahme der gesamten Kiefernschicht ist die Buche zwischen 35 und 55 Jahre alt; es muß mit Schäden in der Buche gerechnet werden. Buchen mit Ernteschäden könnten jedoch in den kommenden Durchforstungen entnommen werden. Die verbleibenden Bäume könnten die Ausfälle ausgleichen. Je weiter sich der Endnutzungszeitraum nach hinten verschiebt, desto wahrscheinlicher ist ein negativer Einfluß auf die Wertentwicklung der Buche. Drittens bleiben Betriebsorganisationskosten bei der Simulation unberücksichtigt. In der Variante ERTRAG ist wegen des hohen und konzentrierten Massenanfalls die Bereitstellung und der Verkauf des Holzes mit weniger Organisationsaufwand durchführbar. Dieser Rückgang der damit verbundenen Fixkosten wird in der Simulation nicht abgebildet.

In der Variante PNV fällt wegen der beiden Durchforstungseingriffe zu Beginn des Simulationszeitraums mit $28 \mathrm{Vfm} /$ ha (über die vierzig Jahre) am wenigsten Totholz aus natürlicher Mortalität an. 50,3Vfm/ha Totholz liefert die Variante LÖWE, in der ein Durchforstungseingriff stattfindet. Am meisten Totholz fällt in der Variante ERTRAG an. Sie liegt mit 79,8Vfm/ha noch vor der Variante PROZESS, die mit 77,2Vfm/ha ebenfalls einen hohen Anteil aufweist. Beiden Varianten ist gemein, daß keine Durchforstung stattgefunden hat und sie nur alle zehn Jahre in den Bestand eingreifen. Dadurch stehen die Bäume in diesen beiden Beständen dichter als in den anderen beiden Varianten. Es kommt zum Absterben von unterständigen Bäumen, die in den Varianten LÖWE und PNV durch die Durchforstungseingriffe begünstigt werden. Daß in der Variante ERTRAG höhere Totholzmengen als in der Variante PROZESS anfallen, liegt daran, daß die früher einsetzenden Erntemaßnahmen in der Variante PROZESS zu einer höheren Überlebenswahrscheinlichkeit der unterständigen Bäume beitragen.

Der Zuwachs der Varianten unterscheidet sich unwesentlich. Der geringe Vorsprung der Variante ERTRAG $(6,8 \mathrm{Vfm} / \mathrm{ha})$ vor den Varianten LÖWE und PNV (beide haben einen Zuwachs von $6,6 \mathrm{Vfm} / \mathrm{ha}$ ) ist durch die Förderung der unterständigen Buchen zu begründen, die in den Variante ERTRAG zu einem früheren Zeitpunkt freigestellt werden. Hinzu kommt, daß in der Variante LÖWE im Jahr 40 der Simulation schon in einem Durchforstungseingriff ein Teil der Buchen entnommen wird. Am geringsten ist der Zuwachs mit $6,4 \mathrm{Vfm} / \mathrm{ha}$ in der Variante PNV. Dies ist mit den beiden Durchforstungseingriffen zu Beginn der Simulation und mit dem Durchforstungseingriff am Ende der Simulationsperiode (in den eingewachsenen Buchen) zu begründen.

Die Unterschiede im Wert des Index-A sind in den Varianten PNV $(0,90)$, LÖWE $(0,89)$ und PROZESS $(0,80)$ durch Zufallseffekte zu erklären. In der Variante ERTRAG liegt der Index-A mit 0,58 deutlich niedriger, da die vollständige Entnahme der führenden Schicht aus Kiefern die Diversität im Bestand absenkt.

Der in der Variante LÖWE markierte Habitatbaum führt rechnerisch am Ende der Simulationsperiode zu einem Habitatbaumvolumen von 12,7Vfm/ha.

Die in den Simulationsläufen eingesetzten Durchforstungs- und Endnutzungsalgorithmen kommen realen Eingriffen in der Praxis nah und führen zu logisch schlüssigen 
Bestandesentwicklungen. Eingriffsart, Eingriffsstärke, Eingriffsintensität und die Zeitpunkte der Eingriffe können nachvollziehbar gesteuert werden.

Die Varianten LÖWE und ERTRAG sehen Pflanzungen von Douglasie mit einer zukünftigen Pflanzfläche von 30 bzw. 100\% der Bestandesfläche vor. Wegen der vorhandenen Buchenverjüngung ist die Durchführung dieser Pflanzungen aus betriebswirtschaftlicher Sicht nicht sinnvoll. Die Werte, die die Zielerreichung der Varianten charakterisieren, ändern sich nur bei den Varianten, die die Buche in ihrem Zieltyp vorsehen. Im Simulationslauf LÖWE ändert sich das Zielerreichungsprozent von 10 auf 70 Prozentpunkte. Sowohl die verbliebenen Kiefern als auch die eingewachsenen Buchen sind im Zieltyp Kiefern-/ Douglasien-/ Buchenmischbestand vorgesehen. Im Simulationslauf PNV steigt das Zielerreichungsprozent von 0 auf 80 Prozentpunkte. Als Zieltyp wird ein Buchenbestand angestrebt.

Tabelle 4.1.2.a: Übersicht über die Ergebnisse der Simulation des Bestandes 4 anhand der Auswertungsparameter.

\begin{tabular}{|c|c|c|c|c|c|c|}
\hline Kriterium & Parameter & Status Quo & $L \ddot{O} W E$ & $P N V$ & ERTRAG & PROZESS \\
\hline \multirow[t]{2}{*}{$\begin{array}{l}\text { Forstliche } \\
\text { Ressourcen }\end{array}$} & $\begin{array}{l}\text { Derbholzvorrat } \\
\text { (Vfm/ha) }\end{array}$ & 378,8 & 345,6 & 264,1 & 124,8 & 331,0 \\
\hline & Abtriebswert (E/ha) & 7626 & 5931 & 3669 & 39 & 5144 \\
\hline \multirow[t]{7}{*}{$\begin{array}{l}\text { Produktions- } \\
\text { funktion }\end{array}$} & $\begin{array}{l}\text { Vornutzungsmasse } \\
\text { (Vfm/ha) }\end{array}$ & $\begin{array}{l}\text { keine } \\
\text { Angabe }\end{array}$ & 63,4 & 143,7 & 0 & 0 \\
\hline & $\begin{array}{l}\text { Endnutzungsmasse } \\
\text { (Vfm/ha) }\end{array}$ & $\begin{array}{l}\text { keine } \\
\text { Angabe }\end{array}$ & 184,0 & 198,1 & 445,8 & 233,55 \\
\hline & $\begin{array}{l}\text { Zuwachs } \\
(\text { Vfm/ha/a) }\end{array}$ & $\begin{array}{l}\text { keine } \\
\text { Angabe }\end{array}$ & 6,6 & 6,4 & 6,8 & 6,6 \\
\hline & $\begin{array}{l}\text { erntekostenfreie Holzerlöse } \\
\text { Vornutzungen } \\
(€ / h a)\end{array}$ & $\begin{array}{l}\text { keine } \\
\text { Angabe }\end{array}$ & 911 & 2791 & 0 & 0 \\
\hline & $\begin{array}{l}\text { erntekostenfreie Holzerlöse } \\
\text { Endnutzungen } \\
(€ / h a)\end{array}$ & $\begin{array}{l}\text { keine } \\
\text { Angabe }\end{array}$ & 4639 & 5079 & 10769 & 6081 \\
\hline & $\begin{array}{l}\text { Pflanzfläche } \\
\text { (\% der Bestandesfläche) }\end{array}$ & $\begin{array}{l}\text { keine } \\
\text { Angabe }\end{array}$ & 0 & 0 & 0 & 0 \\
\hline & $\begin{array}{l}\text { Holzproduktionswert } \\
(\text { (E/ha/a) }\end{array}$ & $\begin{array}{l}\text { keine } \\
\text { Angabe }\end{array}$ & -50 & -18 & -54 & -58 \\
\hline \multirow{3}{*}{$\begin{array}{l}\text { Biologische } \\
\text { Diversität }\end{array}$} & Index- $A$ & 0,41 & 0,89 & 0,90 & 0,58 & 0,80 \\
\hline & $\begin{array}{l}\text { Totholzvolumen } \\
\text { (Vfm/ha) }\end{array}$ & $\begin{array}{l}\text { keine } \\
\text { Angabe }\end{array}$ & 50,3 & 28,0 & 79,8 & 77,2 \\
\hline & $\begin{array}{l}\text { Habitatbaumvolumen } \\
\text { (Vfm/ha) }\end{array}$ & $\begin{array}{l}\text { keine } \\
\text { Angabe }\end{array}$ & 12,7 & 0 & 0 & 0 \\
\hline Zielerreichung & Zielerreichungsprozent (\%) & $\begin{array}{l}\text { LÖWE: } 10 \\
\text { PNV: } 10 \\
\text { ERTRAG: } 0 \\
\text { PROZESS: } \mathrm{x}\end{array}$ & 70 & 80 & 0 & $x$ \\
\hline
\end{tabular}

\subsubsection{Fazit}

Durch die Hintereinanderschaltung von Maßnahmenelementen zu den vier simulationsspezifischen Maßnahmenketten und durch den Rückgriff der Varianten auf spezifische Rahmenwerte nehmen die Beispielbestände deutlich verschiedene 
Entwicklungsrichtungen. Je nach Ausgangssituation kann die Entwicklung der Bestände mehr oder weniger beeinflußt werden. Zum Beispiel bietet der Bestand 1 durch sein Alter und seine Bestandesstruktur erheblich bessere Umbaumöglichkeiten als die Bestände 2 und 3. Durch die Pflanzungsmaßnahmen ist es möglich, einen Umbau der Bestände zu simulieren. Besonderen Einfluß auf die Bestandesentwicklung haben die Pflanzungsmaßnahmen, wenn noch während des Simulationszeitraums Bäume aus diesen Pflanzungen in den Bestand einwachsen (siehe Bestand 1). Bestände, in denen die Verjüngung schon bei Simulationsbeginn festgelegt ist (wie bei Bestand 4 der Fall), oder Bestände, in denen nur relativ wenig Bäume einwachsen (Bestand 2 und Bestand 3), können nur im Rahmen der Möglichkeiten, die die aktuelle Bestockung bietet, umgesteuert werden.

Die Größe der Simulationsbestände ist mit 0,25ha angemessen. Die Bestände können bei dieser Bestandesgröße differenziert auf Eingriffe reagieren. Der Simulationszeitraums von vierzig Jahren scheint geeignet, damit Veränderungen erfaßt und abgebildet werden können.

In den Auswertungsparametern spiegelt sich die unterschiedliche Entwicklung der Bestände in den vier Szenarien wider; die Ergebnisse sind plausibel und nachvollziehbar, auch wenn der Simulationszeitraum eine Einschränkung - gerade im Hinblick auf die Bewertung der Produktionsfunktion - darstellt. Einem Teil der Bestandesentwicklung liegen zufallsgesteuerte Ereignisse zugrunde (wie zum Beispiel dem Einwuchs aus Naturverjüngung). Variantenspezifische Unterschiede werden von diesen Zufallseffekten teilweise nivelliert. Bei der Simulation des Untersuchungsgebiets ist damit zu rechnen, daß sich die zufallsbedingten Einflüsse auf den Einzelbestand in der Menge der simulierten Bestände ausgleichen.

\subsection{Auswertung des Ist-Zustands der Landschaft}

Von der speziellen Betrachtung auf Bestandesebene kommend, befaßt sich das nun anschließende Kapitel auf regionaler Ebene mit dem Untersuchungsgebiet selbst. In den anschließenden Unterkapiteln werden die Werte der Auswertungsparameter im Status Quo vorgestellt. Auf die Definition und Herleitung dieser Werte wurde in Kapitel 3.6 eingegangen. Die Parameter der Produktionsfunktion (wie Nutzungsmassen und Zuwächse) sind nicht Teil dieser Auswertung, da es sich um eine Zustandsbeschreibung ohne zeitlich vorgelagerten Bezugspunkt handelt. Die Entwicklung, die zu dieser Ausgangssituation geführt hat, kann in der Analyse des Ausgangszustands nicht nachvollzogen werden.

Da sich aus den Forsteinrichtungsdaten weder das in den letzten Jahren angefallene Totholzvolumen noch eine Information über das Volumen der ausgewiesenen Habitatbäume (gemäß der in Kapitel 3.6.2 beschriebenen Definitionen) ableiten lassen, können diese Werte auch nicht bei der Auswertung des Ist-Zustand berücksichtigt werden. 


\subsubsection{Forstliche Ressourcen}

Der Derbholzvorrat liegt zu Beginn der Simulation durchschnittlich lediglich bei $192 \mathrm{Vfm} / \mathrm{ha}$ und konzentriert sich mit $56 \%$ seines Volumens besonders auf die Durchmesserklassen von 15 bis $30 \mathrm{~cm}$ (siehe Abbildung 4.2.1.a).

Bedingt durch den hohen Anteil von Bäumen mit geringem Durchmesser liegt der erntekostenfreie Abtriebswert auf ca. 20\% der Fläche des Untersuchungsgebiets im negativen Bereich. Insgesamt beträgt der Mittelwert aus den erntekostenfreien Abtriebswerten der Bestände 1887€/ha.

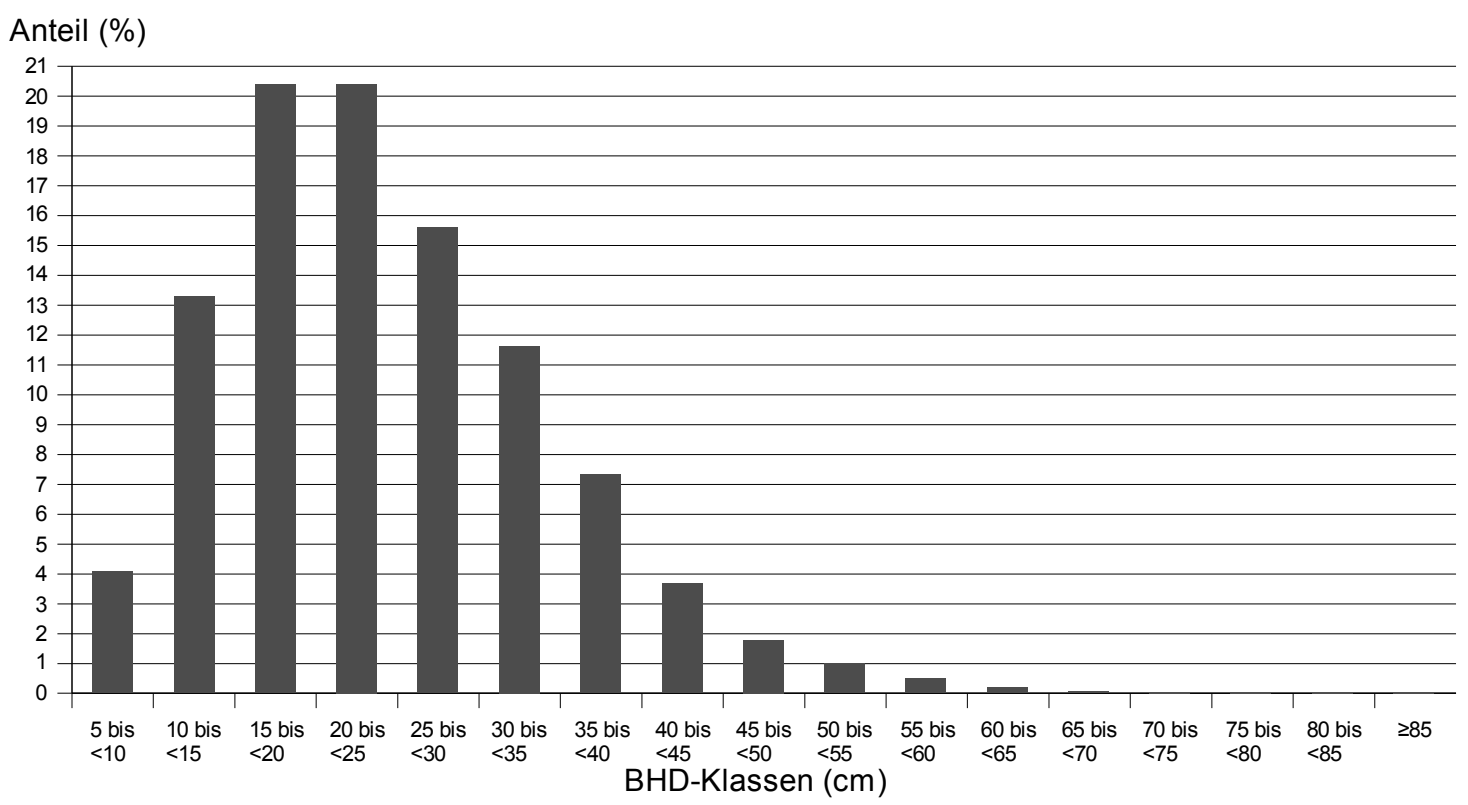

Abbildung 4.2.1.a: Prozentualer Volumenanteil der BHD-Klassen am Gesamtvorrat des Untersuchungsgebiets.

\subsubsection{Biologische Diversität}

Die landschaftliche Vielfalt liegt mit einem Durchmischungskoeffizienten von 1,4285 bei $87 \%$ des maximal möglichen Koeffizienten (1,6367). Dieser, unter Beibehaltung der heutigen Bestandesgrenzen maximal mögliche Durchmischungskoeffizient ist erreicht, wenn alle Bestände des Untersuchungsgebiets von Beständen umgeben sind, die einem Bestandestyp zugeordnet werden, der nicht dem ihrigen entspricht (Definition siehe auch Kapitel 3.6.2).

Der Index-A, ein Maß für die Diversität in den einzelnen Beständen, beträgt im Status Quo im Mittel 0,80. Bei der Interpretation dieses Werts muß beachtet werden, daß der Bestandesaufbau stark durch das Aufnahmeverfahren geprägt ist. Wenn zum Beispiel Arten methodisch bedingt nicht aufgenommen werden, wird der Index-A in der Auswertung des Status Quo unterschätzt. Zu einer Überschätzung des Index-A kann es dagegen nicht kommen, ein einseitig methodischer Fehler ist möglich. Um so detaillierter der Bestand vom Forsteinrichter aufgenommen wurde, desto größer und realitätsgetreuer wird in der Regel auch der Index-A. 


\subsubsection{Zielerreichung}

Die Verteilung der Bestandestypengruppen wurde schon bei der Beschreibung des Untersuchungsgebiets vorgestellt (siehe Abbildung 3.2.1.b). Untersucht man die Baumartenzusammensetzung innerhalb der Bestände im einzelnen, so kann sie mit den angestrebten Mischungsverhältnissen der vier Varianten verglichen werden. Für jede der vier Varianten ergibt sich ein Zielerreichungsprozent im Status Quo aus der Differenz der Artenzusammensetzung im Status Quo zu dem durch den jeweiligen Zieltyp festgelegten Mischungsverhältnis (siehe Tabelle 4.2.3.a).

Tabelle 4.2.3.a: Zielerreichungsprozent zu Beginn der Simulation für die Varianten LÖWE, PNV und ERTRAG. In der Variante PROZESS wird kein Ziel zur Baumartenzusammensetzung angestrebt, daher kann für sie keine Angabe gemacht werden.

\begin{tabular}{lc}
\hline Variante & $\begin{array}{c}\text { Zielerreichung (\%) } \\
\text { zu Beginn der Simulation }\end{array}$ \\
\hline LÖWE & 48 \\
\hline $\boldsymbol{P N V}$ & 18 \\
\hline ERTRAG & 42 \\
\hline
\end{tabular}

In der Variante PROZESS wird kein Ziel zur Baumartenzusammensetzung angestrebt, sie fällt aus dieser Betrachtung heraus. Die Baumartenmischung im Status Quo entspricht am meisten der Zielzusammensetzung der Variante LÖWE, sie ist zu 48\% erreicht. Aber auch das Ziel der Variante ERTRAG ist schon zu Beginn der Simulation zu 42\% umgesetzt. Für die Variante PNV entsprechen lediglich $18 \%$ der derzeitigen Bestockung dem angestrebten Mischungsverhältnis.

\subsubsection{Sonstiges}

Bei der Aufbereitung der Daten des Status Quo wird auf Forsteinrichtungsdaten zurückgegriffen, die weitgehend nach der „Anweisung zur Betriebsregelung (Forsteinrichtung) in den Niedersächsischen Landesforsten (B.A.87)“ (NiEDERSÄCHSISCHE LANDESFORSTVERWALTUNG 1987) aufgenommen wurden. Der Dg wird im Rahmen dieses Aufnahmeverfahrens nicht im Bestand erhoben, sondern über Hilfstafeln (Niedersächsisches Forstplanungsamt 1987) aus den Ertragstafeln (zum Beispiel Schober 1975) hergeleitet. Auch das im Rahmen dieser Arbeit gewählte Datenergänzungsverfahren bei fehlender Angabe des Dg im Ausgangsdatensatz greift auf diese Hilfstafeln zurück. In ihrer Veröffentlichung weisen Peter Wollborn und Thomas Böckmann darauf hin, daß „der Ertragstafeldurchmesser i.d.R. auf Grund der stärkeren Behandlungsregime der letzten Dekaden vom realen Bestandesdurchmesser deutlich abweicht“ (WollbORN UND BöCKMANN 1998). Da jedoch alle vier Simulationsvarianten auf dieser den Dg unterschätzenden Datengrundlage basieren, bleiben die Ergebnisse zwischen den Varianten vergleichbar. 


\subsection{Szenarienergebnisse im Vergleich}

Die Ergebnisse der vier Szenarien (siehe Tabelle 4.3.a) können einerseits in bezug zum Status Quo andererseits aber auch untereinander verglichen werden.

Tabelle 4.3.a: Übersicht über die Ergebnisse der Simulation anhand der Auswertungsparameter.

\begin{tabular}{|c|c|c|c|c|c|c|}
\hline Kriterium & Parameter & Status Quо & $L \ddot{O} W E$ & $P N V$ & ERTRAG & PROZESS \\
\hline \multirow[t]{3}{*}{$\begin{array}{l}\text { Forstliche } \\
\text { Ressourcen }\end{array}$} & $\begin{array}{l}\text { Derbholzvorrat } \\
(\mathrm{Vfm} / \mathrm{ha})\end{array}$ & 192 & 344 & 259 & 337 & 346 \\
\hline & Durchmesserstruktur & $\begin{array}{l}\text { siehe Abb. } \\
\text { 4.2.1.a }\end{array}$ & $\begin{array}{l}\text { siehe Abb. } \\
\text { 4.3.1.a }\end{array}$ & $\begin{array}{l}\text { siehe Abb. } \\
\text { 4.3.1.b }\end{array}$ & $\begin{array}{l}\text { siehe Abb. } \\
\text { 4.3.1.c }\end{array}$ & $\begin{array}{l}\text { siehe Abb. } \\
\text { 4.3.1.d }\end{array}$ \\
\hline & Abtriebswert (€/ha) & 1887 & 5929 & 4301 & 5410 & 5930 \\
\hline \multirow[t]{7}{*}{$\begin{array}{l}\text { Produktions- } \\
\text { funktion }\end{array}$} & $\begin{array}{l}\text { Vornutzungsmasse } \\
\text { (Vfm/ha) }\end{array}$ & keine Angabe & 150 & 222 & 144 & 125 \\
\hline & $\begin{array}{l}\text { Endnutzungsmasse } \\
\text { (Vfm/ha) }\end{array}$ & keine Angabe & 32 & 36 & 45 & 40 \\
\hline & $\begin{array}{l}\text { Zuwachs } \\
(\text { Vfm/ha/a) }\end{array}$ & keine Angabe & 9,8 & 9,0 & 10,1 & 9,7 \\
\hline & $\begin{array}{l}\text { erntekostenfreie } \\
\text { Holzerlöse } \\
\text { Vornutzungen } \\
(€ / h a)\end{array}$ & keine Angabe & 1831 & 2849 & 1815 & 1817 \\
\hline & $\begin{array}{l}\text { erntekostenfreie } \\
\text { Holzerlöse } \\
\text { Endnutzungen } \\
(€ / h a) \\
\end{array}$ & keine Angabe & 1075 & 1190 & 1428 & 1382 \\
\hline & $\begin{array}{l}\text { Pflanzfläche } \\
\text { (\% der Bestandesfläche) }\end{array}$ & keine Angabe & 18 & 45 & 22 & 0 \\
\hline & $\begin{array}{l}\text { Holzproduktionswert } \\
(\text { E/ha/a) }\end{array}$ & keine Angabe & 67 & 1 & 59 & 90 \\
\hline \multirow[t]{4}{*}{$\begin{array}{l}\text { Biologische } \\
\text { Diversität }\end{array}$} & $\begin{array}{l}\text { Durchmischungs- } \\
\text { koeffizient }\end{array}$ & 1,43 & 1,51 & 1,47 & 1,5 & 1,49 \\
\hline & Index $-A$ & 0,80 & 0,97 & 0,86 & 0,89 & 0,98 \\
\hline & $\begin{array}{l}\text { Totholzvolumen } \\
\text { (Vfm/ha) }\end{array}$ & keine Angabe & 57,8 & 37,6 & 69,8 & 70,9 \\
\hline & $\begin{array}{l}\text { Habitatbaumvolumen } \\
\text { (Vfm/ha) }\end{array}$ & keine Angabe & 1,7 & 0 & 0 & 2,0 \\
\hline \multirow[t]{2}{*}{ Zielerreichung } & $\begin{array}{l}\text { Zielerreichungsprozent } \\
\text { (\%) }\end{array}$ & $\begin{array}{l}\text { LÖWE: } 48 \\
\text { PNV: } 18 \\
\text { ERTRAG: } 42 \\
\text { PROZESS: } \mathrm{x}\end{array}$ & 58 & 49 & 47 & $x$ \\
\hline & $\begin{array}{l}\text { Verteilung der } \\
\text { Bestandestypen }\end{array}$ & $\begin{array}{l}\text { siehe Abb. } \\
\text { 3.2.1.b }\end{array}$ & $\begin{array}{l}\text { siehe Abb. } \\
\text { 4.3.4.b }\end{array}$ & $\begin{array}{l}\text { siehe Abb. } \\
\text { 4.3.4.b }\end{array}$ & $\begin{array}{l}\text { siehe Abb. } \\
\text { 4.3.4.b }\end{array}$ & $\begin{array}{l}\text { siehe Abb. } \\
\text { 4.3.4.b }\end{array}$ \\
\hline
\end{tabular}

In den Kapiteln 4.3.1 bis 4.3.4 werden sie anhand der Auswertungsparameter (siehe Kapitel 3.6) beschrieben und diskutiert. Die Auswertung ist in die Handlungsfelder „Forstliche Ressourcen“, „Produktionsfunktion“, „Biologische Diversität“ und den Bereich „Zielerreichung“ gegliedert. Das Kapitel 4.3.5 fast die Ergebnisse bereichsübergreifend zu einer vergleichenden Übersicht zusammen, die eine abschließende Bewertung der Szenarienläufe (Kapitel 4.3.6) ermöglicht. 


\subsubsection{Forstliche Ressourcen}

Wie in Kapitel 3.2.1 vorgestellt wurde, befindet das Untersuchungsgebiet sich in der Phase des Vorratsaufbaus, denn im untersuchten Waldgebiet dominiert die zweite und vierte Altersklasse (siehe Abbildung 3.2.1.c). Diese Situation spiegelt sich auch in den forstlichen Ressourcen wider.

Der Derbholzvorrat steigt in allen vier Varianten im Vergleich zum Status Quo an (siehe Tabelle 4.3.a). Dabei liegen die Varianten LÖWE, ERTRAG und PROZESS mit Werten zwischen 337 und $346 \mathrm{Vfm} /$ ha relativ eng beieinander; nur in der Variante PNV baut sich mit 259Vfm/ha ein weit geringerer Vorrat auf. Dieser Trend war schon bei den Beispielbeständen 1-3 zu beobachten und ist durch die häufigen Eingriffe mit hohem Freistellungsgrad zu erklären. Untersucht man den Verlauf des Vorratsaufbaus (ohne Abbildung) näher, so stellt man fest, daß gerade im ersten Durchforstungseingriff in der Variante PNV der Vorrat vergleichsweise stark herabgesenkt wird. Diese Vorratsreduktion wirkt sich negativ auf die weitere Entwicklung der Bestandesmasse aus.

Die Durchmesserstruktur verschiebt ihren Schwerpunkt in allen drei Varianten von den Durchmesserklassen zwischen 15 und $30 \mathrm{~cm}$ hin zu den Durchmesserklassen zwischen 25cm und 45cm BHD (siehe Abbildungen 4.3.1.a bis 4.3.1.d).

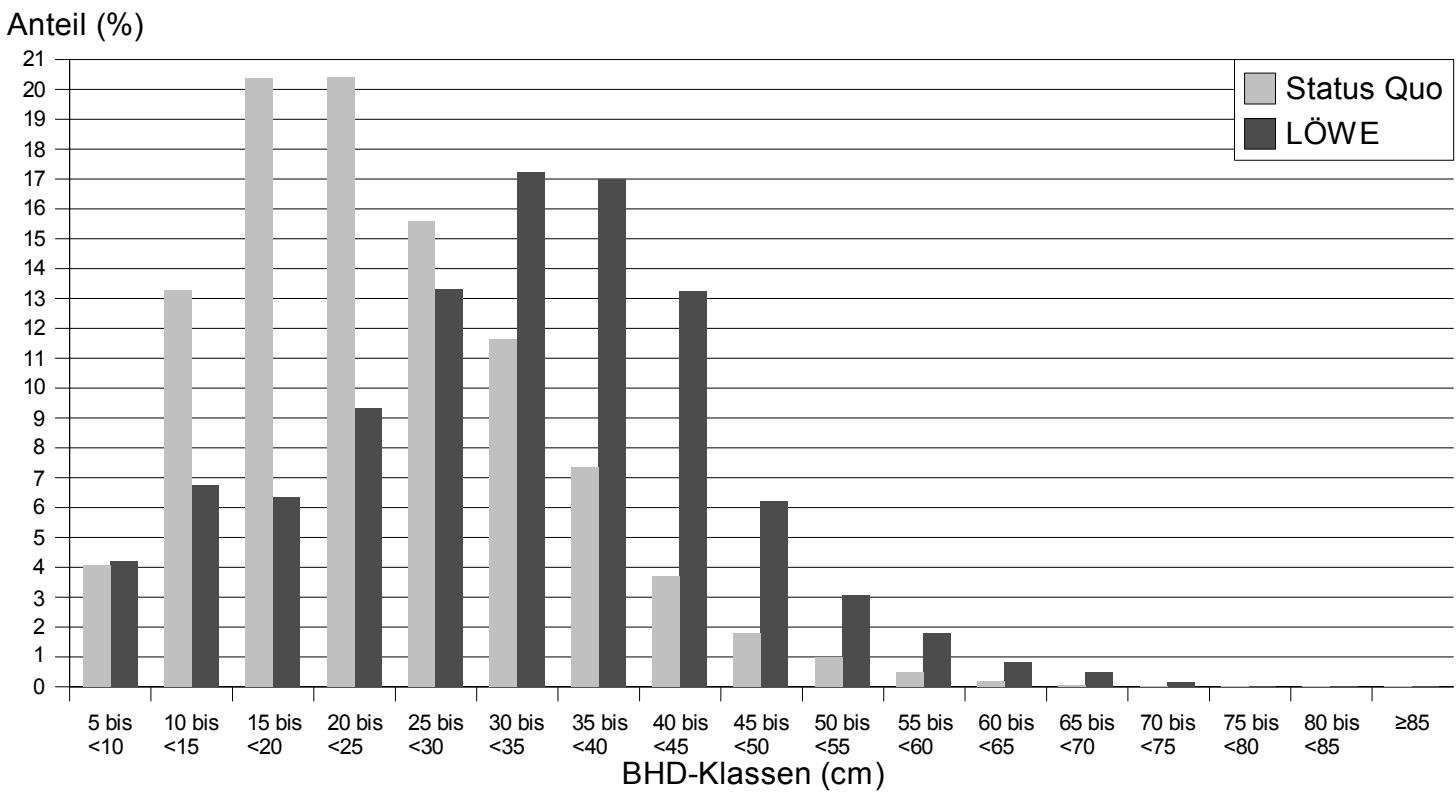

Abbildung 4.3.1.a: Prozentualer Volumenanteil der BHD-Klassen am Gesamtvorrat des Untersuchungsgebiets nach der vierzigjährigen Simulation der Variante LÖWE im Vergleich zum Status Quo. 


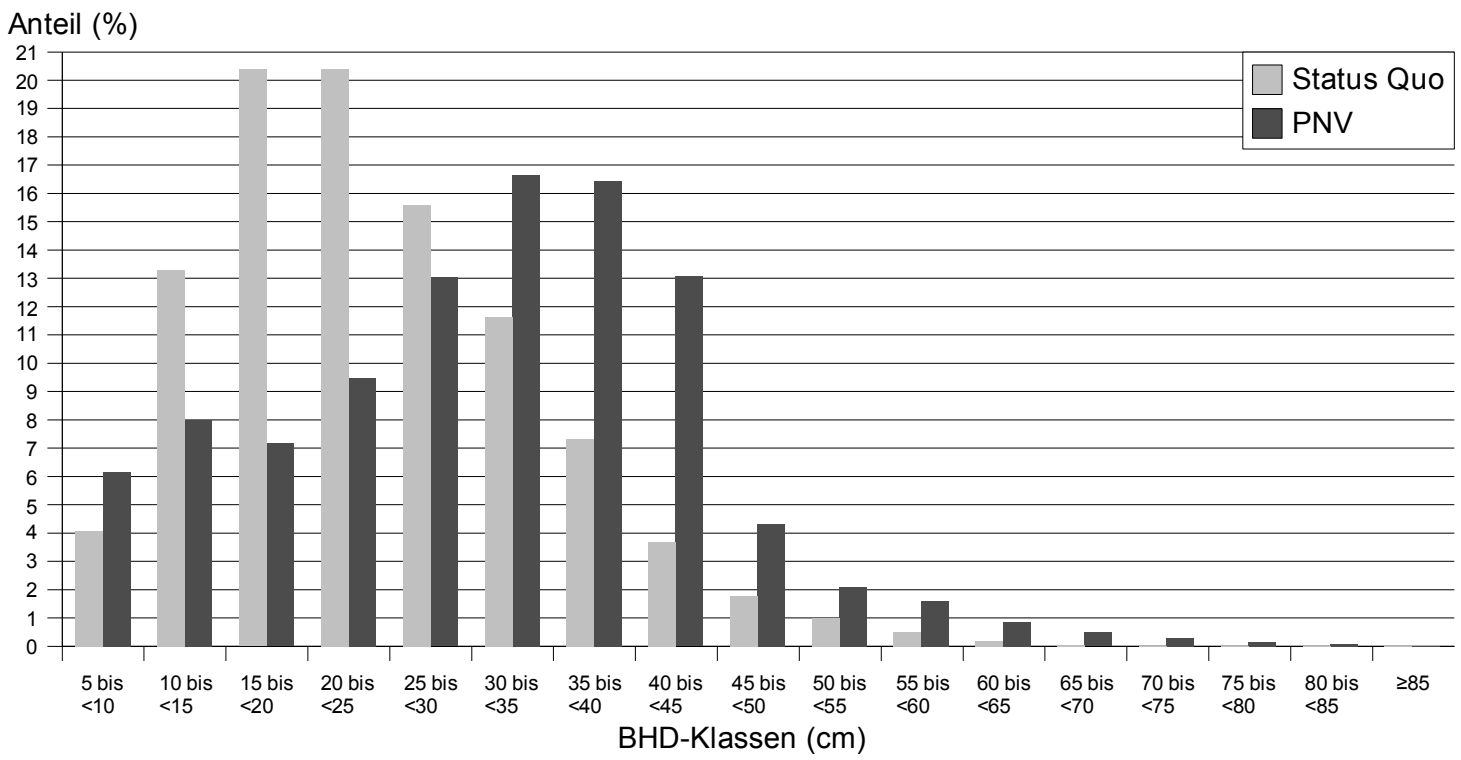

Abbildung 4.3.1.b: Prozentualer Volumenanteil der BHD-Klassen am Gesamtvorrat des Untersuchungsgebiets nach der vierzigjährigen Simulation der Variante PNV im Vergleich zum Status Quo.

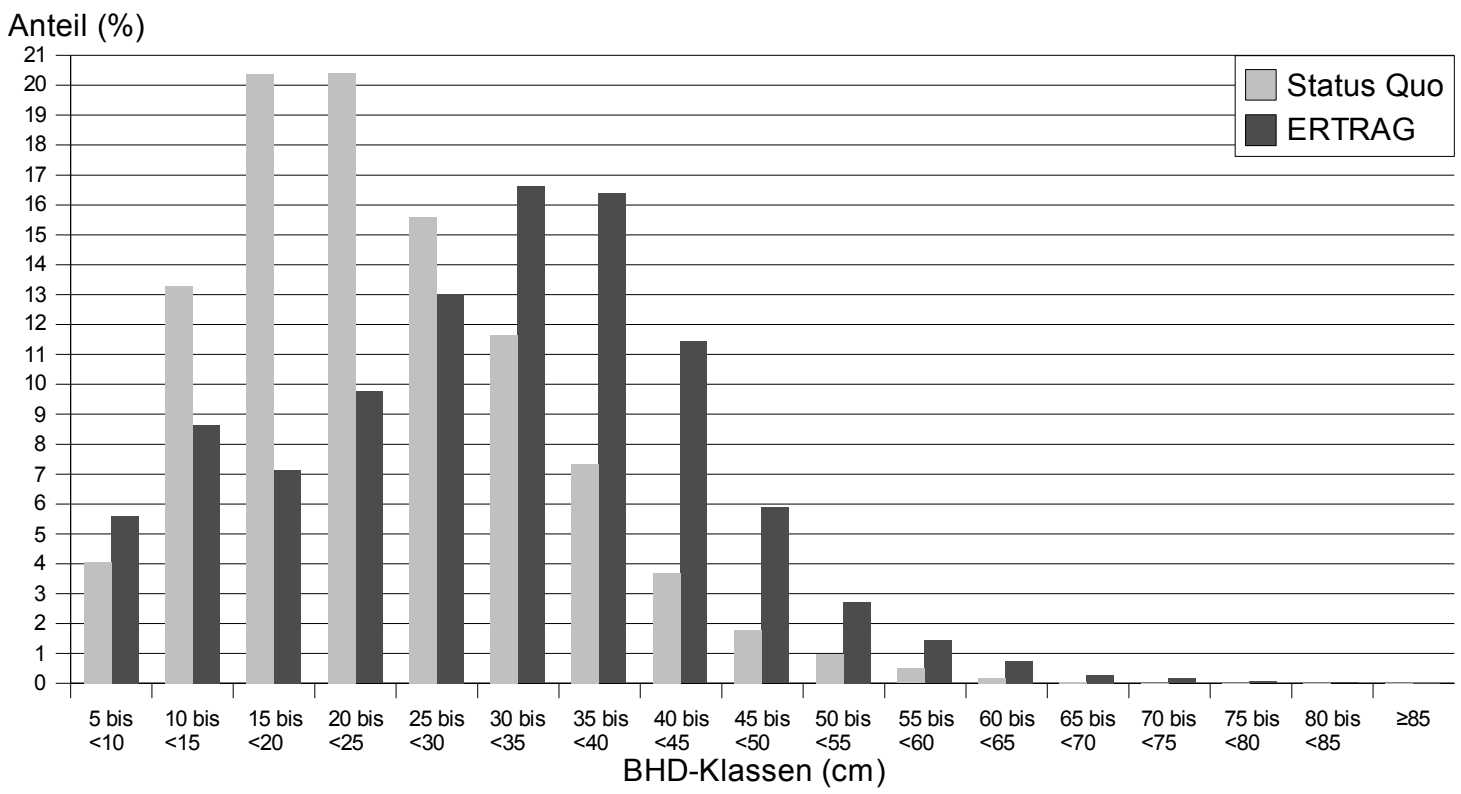

Abbildung 4.3.1.c: Prozentualer Volumenanteil der BHD-Klassen am Gesamtvorrat des Untersuchungsgebiets nach der vierzigjährigen Simulation der Variante ERTRAG im Vergleich zum Status Quo. 


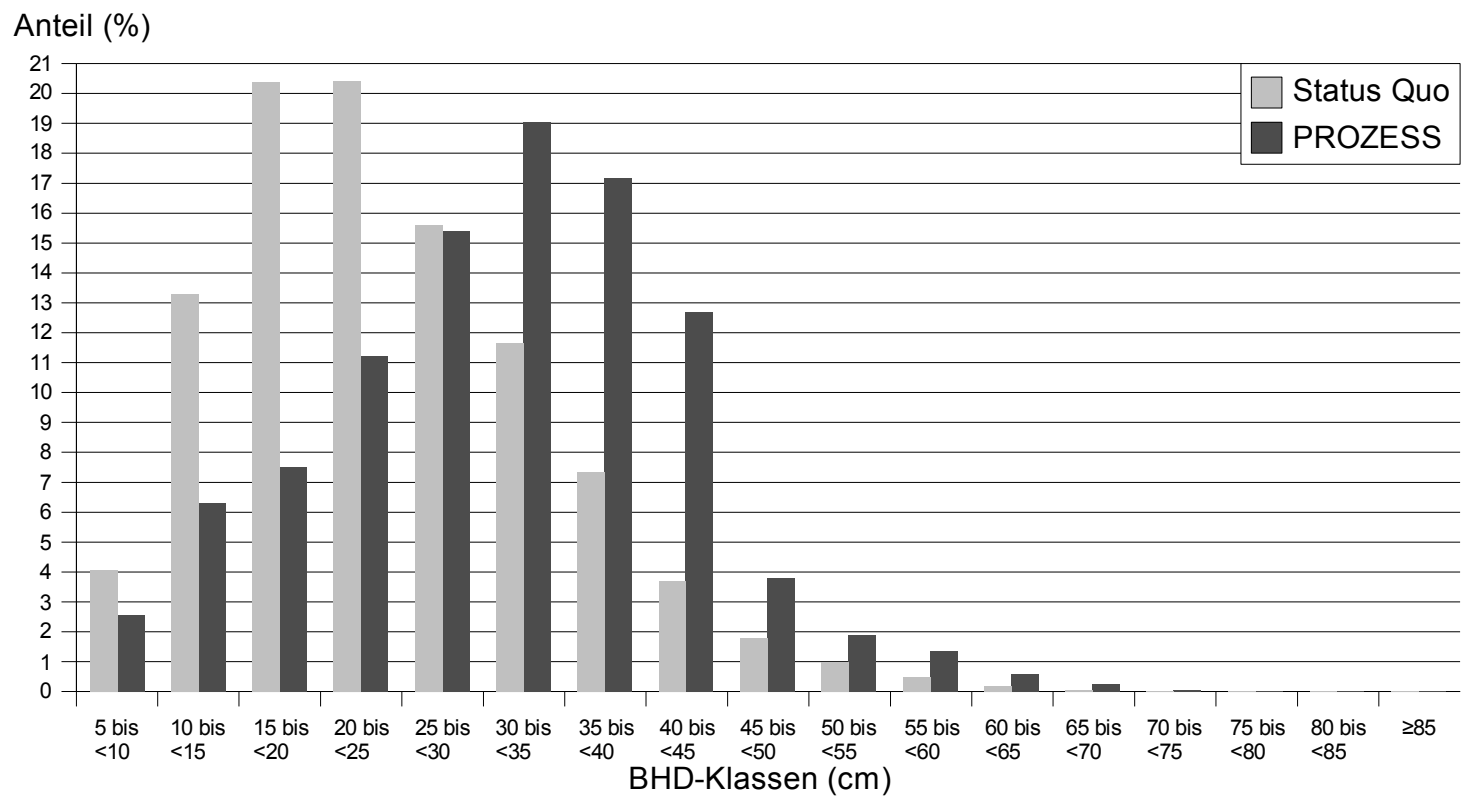

Abbildung 4.3.1.d: Prozentualer Volumenanteil der BHD-Klassen am Gesamtvorrat des Untersuchungsgebiets nach der vierzigjährigen Simulation der Variante PROZESS im Vergleich zum Status Quo.

In allen Varianten wird hochdurchforstungsartig eingegriffen, was zur Ausbildung eines schwächeren zweiten Peaks in der Durchmesserklasse $10 \mathrm{~cm}-15 \mathrm{~cm}$ BHD führt. Die Variante ERTRAG akkumuliert mit ca. $11 \%$ des Gesamtvorrates weniger Masse in der Durchmesserklasse von $40 \mathrm{~cm}-45 \mathrm{~cm}$ BHD als die anderen Varianten, bei denen sich ungefähr $13 \%$ des Vorrates in dieser Klasse befinden. Das liegt daran, daß der Zieldurchmesser der im Untersuchungsgebiet dominierenden Kiefer in der Variante ERTRAG bei $40 \mathrm{~cm}$ liegt. In den anderen Varianten werden die Bäume erst beim Erreichen eines Zieldurchmessers von $45 \mathrm{~cm}$ endgenutzt. Der Effekt wird durch die zeitliche Begrenzung der Endnutzung auf zwanzig Jahre bei der Variante ERTRAG noch verstärkt.

In den kommenden Jahrzehnten verschiebt sich der Schwerpunkt der Durchmesserverteilung weiter nach rechts, hin zu den höheren BHD-Klassen. In allen vier Varianten werden daher verstärkt Endnutzungssortimente anfallen.

Der erntekostenfreie Abtriebswert (siehe Tabelle 4.3.a) des verbleibenden Bestandes steigt mit dem Vorratsaufbau der Bestände und der Verschiebung der Durchmesserverteilung in höhere BHD-Klassen an. Am höchsten ist er mit 5930€/ha in der Variante PROZESS. Allerdings ist in dieser Variante zu beachten, daß keine kontinuierliche Z-Baum-Förderung durchgeführt wird, da gemäß der Maßnahmenkette PROZESS (siehe Kapitel 3.5.4) nur temporäre Z-Bäume ausgewählt werden. Die durchschnittliche Qualität der Bestände PROZESS verschlechtert sich demnach im Vergleich zu den anderen Varianten über die Jahre. Diese Verschlechterung wurde in den Erlösfunktionen berücksichtigt und wird sich in den kommenden Jahren noch weiter verstärken. Auch profitiert die Entwicklung der Bestände innerhalb der Simulationsperiode von den Pflanzungen, die zu Beginn des Simulationszeitraums schon getätigt worden waren. Für die Zukunft ist zu erwarten, daß durch Vergrasung in Kiefernaltbeständen und 
durch das verstärkte Aufkommen von Weichlaubhölzern die Bestände in ihrer Wertentwicklung nicht mit den anderen drei Varianten mithalten können. Knapp unter dem Abtriebswert am Ende der Simulationsperiode der Variante PROZESS liegt der Wert der Bestände der Variante LÖWE mit 5929€/ha, gefolgt von der Variante ERTRAG $(5410 € / h a)$. Besonders in den Varianten LÖWE und ERTRAG ist zu erwarten, daß die Bestände in Zukunft von den Pflanzungen profitieren. Die zeitlich begrenzte Endnutzungsstrategie der Variante ERTRAG kann jedoch in dieser Variante zu einem Absinken der Abtriebswerte führen, wenn weitere Teile des Untersuchungsgebiets erntereif werden. Bei der Zielstärkennutzung ist dieser Effekt in den Varianten LÖWE und PNV nicht zu erwarten.

Die Bestände der Variante PNV haben mit 4301€/ha den geringsten Abtriebswert. Wie sich schon in den Beispielbeständen zeigte, werden in dieser Variante im Rahmen der Umsteuerung der Bestände hin zur PNV hohe Mengen bei Durchforstungen entnommen, was den Derbholzvorrat und damit auch den erntekostenfreien Abtriebswert nachhaltig beeinflußt.

\subsubsection{Produktionsfunktion}

Die Variante PNV greift in den Vornutzungen wesentlich stärker in die Bestände ein (eine Vornutzungsmasse von 222Vfm/ha fällt in vierzig Jahren) als die anderen drei Varianten. Das Bild, das bei der Betrachtung der Vornutzungen der Beispielbestände entsteht, wird im Untersuchungsgebiet bestätigt. In der Variante LÖWE werden durchschnittlich $150 \mathrm{Vfm} / \mathrm{ha}$ in vierzig Jahren entnommen, in der Variante ERTRAG $144 \mathrm{Vfm} / \mathrm{ha}$ und in der Variante PROZESS $125 \mathrm{Vfm} / \mathrm{ha}$.

Die Summe der erntekostenfreien Holzerlöse aus Vornutzungen sind weniger von dem Wert des entnommenen Holzes, als vielmehr von der Masse abhängig. In der Variante PNV kommt man auf Erlöse von $2849 € /$ ha, die anderen Varianten liegen bei $1800 € /$ ha. Dabei fällt auf, daß die Variante PROZESS einen ca. $2 €$ höheren Wert pro Vorratsfestmeter erzielt (ohne direkte Abbildung in der Tabelle). Bei den seltenen, schwachen Eingriffen werden demnach bevorzugt stärkere Bäume entnommen. Diskontiert man die Erträge aus der Vornutzung, so relativiert sich der höhere Erlös pro Festmeter durch die späteren Eingriffszeitpunkte der Variante PROZESS wieder. Er liegt dann sogar ein paar Cent unter dem der Varianten PNV und ERTRAG (ohne Abbildung in der Tabelle).

Die Endnutzungsmasse ist in der Variante ERTRAG erwartungsgemäß am höchsten. Sie beträgt als Summe über den Simulationszeitraum 45Vfm/ha im Vergleich zu 40Vfm/ha in der Variante PROZESS, 36Vfm/ha in der Variante PNV und 32Vfm/ha in der Variante LÖWE. Da nicht immer die gesamte Endnutzungsphase eines Bestandes in den Simulationszeitraum fällt, ist die Differenz zwischen den Varianten geringer, als sie im Beispiel 4, dem Beispiel für einen Endnutzungsbestand, ausfiel.

In der Variante ERTRAG wird mit $31,70 €$ ein geringerer Durchschnittserlös pro Vorratsfestmeter (ohne Abbildung in der Tabelle) in der Endnutzung erzielt als in den anderen drei Varianten. Dort liegen die Werte zwischen $33 €$ (Variante PNV) und 34,60€ 
(Variante PROZESS). Der Grund dafür ist die durch niedrigere Zielstärken bedingte frühere Endnutzung. In der Summe der erntekostenfreien Holzerlöse aus Endnutzungen liegt die Variante ERTRAG jedoch wegen der hohen Entnahmemengen mit $1428 € /$ ha vor den anderen Varianten (PROZESS: 1382€/ha, PNV: 1190€/ha und LÖWE: $1075 € / h a)$.

Der Zuwachs ist in der Variante PNV am niedrigsten. Er beträgt nur 9 Vorratsfestmeter pro Hektar und Jahr, da die starken Durchforstungseingriffe zu nachhaltigen Zuwachsverlusten führen. Am höchsten ist der Zuwachs in der Variante ERTRAG $(10,1 \mathrm{Vfm} / \mathrm{ha})$. Einerseits begünstigt der starke Freistellungsgrad den Zuwachs der geförderten Bäume. Andererseits wirkt sich der Einwuchs von Douglasien und Fichten, die auf großen Teilen des Untersuchungsgebiets gepflanzt wurden, positiv auf den Zuwachs aus. Die Varianten LÖWE und PROZESS liegen mit 9,8 bzw. 9,7Vfm/ha/a zwischen den genannten Werten. In der Variante PROZESS wird dieser Wert durch den hohen Vorrat erreicht. Bei der Variante LÖWE ist er eher auf die permanente Förderung der Z-Bäume und durch den Einwuchs von Bäumen aus Pflanzungen zurückzuführen.

Zieht man den Holzproduktionswert als Anhaltspunkt für das betriebswirtschaftliche Gesamtergebnis der vier Varianten heran, so schneidet die Variante PROZESS mit $90 € / \mathrm{ha} / \mathrm{a}$ am besten ab. Es folgen die Varianten LÖWE (67€/ha/a) und ERTRAG (59€/ha/a). Am niedrigsten fällt der Wert bei der Variante PNV mit $1 € /$ ha/a aus. Die Variante PROZESS profitiert im Simulationszeitraum davon, daß sie keinerlei Pflanzungsmaßnahmen vorsieht. Der prozentuale Anteil der Pflanzfläche am gesamten Untersuchungsgebiet und die Baumartenwahl bei der Pflanzung beeinflussen das Ergebnis in entscheidendem Maße. Betrachtet man den Holzproduktionswert, ohne die Pflanzungskosten zu berücksichtigen, ergibt sich ein anderes Verhältnis unter den Varianten (ohne Abbildung in der Tabelle). Einerseits liegen die Werte für den Holzproduktionswert enger beieinander, andererseits verschiebt sich die Reihenfolge der Varianten. Die Variante PROZESS bildet mit $90 €$ das Schlußlicht hinter den Varianten ERTRAG (93€/ha/a), LÖWE (95€/ha/a) und PNV (97€/ha/a).

Am höchsten ist der Anteil der Pflanzungen in der Variante PNV mit einer Pflanzfläche von 45\%. Dieser hohe Anteil erklärt den großen Unterschied zwischen dem Holzproduktionswert mit und ohne Pflanzungskosten. Die Pflanzaufwendungen sind in dieser Variante dadurch bedingt, daß die Baumartenmischung im Status Quo vom Baumartenmischungsziel der Variante PNV weiter entfernt ist, als dies in den Varianten LÖWE und ERTRAG der Fall ist (siehe Kapitel 4.3.4, Zielerreichung). Auch die starke Umsteuerung mit Hilfe der Durchforstungsmaßnahmen und die zu Beginn der Simulation vorhandene Buchenverjüngung kann diese Pflanzungen nicht ersetzen. (Zu Beginn der Simulation existiert laut Forsteinrichtung auf ca. $7 \%$ der Fläche eine gesicherte Buchenverjüngung, die größtenteils aus Pflanzungen stammt.)

In den beiden anderen Strategien, die Pflanzungen vorsehen, wird nicht einmal die Hälfte des Pflanzaufwands der Variante PNV betrieben. Daher sind die Auswirkungen auf den Holzproduktionswert nicht so stark wie in der Variante PNV. Der Unterschied der Pflanzungen in den Varianten ERTRAG und LÖWE liegt weniger in der bepflanzten Fläche $(22 \%$ bzw. 18\%) als vielmehr in der Artenzusammensetzung, die gepflanzt wird. Werden in 
der Variante ERTRAG fast ausschließlich Nadelbaumarten gepflanzt, so arbeitet die Variante LÖWE mit einer Mischung aus Nadel- und Laubbäumen, wobei der Laubholzanteil überwiegt. Die Pflanzung von Nadelbaumarten ist mit tendenziell geringeren Pflanzungskosten verbunden, so daß die Summe der Pflanzungskosten in den beiden Varianten ähnlich hoch liegt.

Um den betriebswirtschaftlichen Erfolg der Durchforstungs- und Endnutzungsstrategien zu beurteilen, werden daher im folgenden die Holzproduktionswerte ohne Pflanzung untersucht. Die Variante PNV unterscheidet sich besonders in der Eingriffsstärke bei Durchforstungen von den anderen Varianten. In den Vornutzungen fällt daher wesentlich mehr Ertrag an als in den anderen drei Varianten. Da jedoch der Abtriebswert der PNVBestände durch die hohen Entnahmen geringer ausfällt, liegt der Holzproduktionswert der Variante PNV nur etwa $3 €$ pro Hektar höher als in der Variante LÖWE. Die Variante LÖWE schafft es durch ihren als „normal“ eingestuften Freistellungsgrad, die Bestände bei einem relativ hohen Derbholzvorrat wertsteigernd zu differenzieren.

Beim Abschneiden der Variante ERTRAG, die lediglich den vorletzten Rang in bezug auf den Holzproduktionswert ohne Pflanzungskosten belegt, bleiben mehrere betriebswirtschaftliche Faktoren bei der Auswertung unberücksichtigt. Die Stärke dieser Variante sind Einsparpotentiale, die sich durch seltene, starke Eingriffe ergeben, denn die Kosten der Holzernte unterliegen ebenso wie die meisten anderen Kosten eines produktiven Betriebs einer Auflagendegression. Die Simulation berücksichtigt Vorzüge, die die Variante durch die Nutzung größerer Hiebsflächen, den Anfall höherer Erntemengen und selteneren Eingriffen bietet, jedoch nicht. Und selbst wenn diese Faktoren durch Korrekturfaktoren mit in die Simulation einfließen würden, käme die Stärke der Variante ERTRAG, die besonders in der Endnutzungsphase liegt, in dem untersuchten Gebiet nicht voll zur Geltung: Die Bestände des Untersuchungsgebiets befinden sich derzeit noch in einer Phase des Vorratsaufbaus, der Großteil der Endnutzungen fällt erst in den nächsten Jahrzehnten nach Ende der Simulationsperiode an. Weiterhin ist die Endnutzungsstrategie dieser Variante darauf ausgerichtet, die Umtriebszeiten zu verkürzen. Dieser Effekt kann bei einer Beschränkung des Simulationszeitraums auf vierzig Jahre nur unzulänglich abgebildet werden. Ein weiterer Aspekt ist der von der Variante ERTRAG forcierte Baumartenwechsel. Die Pflanzung von ertragsstarken Baumarten, wie der Douglasie wird sich positiv auf das Betriebsergebnis auswirken. Schon in der Durchforstungsphase wird dieser Effekt bemerkbar sein. In der Endnutzungsphase kommt er dann voll zum Tragen.

Die Durchforstungs- und Endnutzungsstrategie der Variante PROZESS führt im Vergleich zu den anderen Varianten zu dem niedrigsten Holzproduktionswert. Zwar werden in der Variante wegen der seltenen und schwachen Eingriffe tendenziell eher Bäume mit einem höheren Durchmesser und somit auch einem höheren Wert als in den anderen Varianten entnommen, aber dieser Vorteil reduziert sich durch die späten Eingriffszeitpunkte. Da der Holzproduktionswert den Zeitpunkt berücksichtigt, zu dem die Erlöse anfallen, schneidet die Variante schlechter als die anderen drei Varianten ab. Hinzu kommt, daß durch die diskontinuierliche Pflege der Bäume (Z-Bäume werden nicht permanent markiert) die Wertentwicklung der Bestände im Laufe der Jahre hinter der der anderen Varianten immer weiter zurückbleiben wird. Desweiteren profitiert diese Variante derzeit noch von den Pflanzungen, die schon zu Simulationsbeginn angelegt worden waren. Ohne diese 
Pflanzungen und ohne Bodenbearbeitungsmaßnahmen werden Weichlaubhölzer einen immer größeren Anteil in der Baumartenzusammensetzung einnehmen, und der Wert der Bestände vermindert sich weiter.

Für die zukünftige Entwicklung der Bestände bietet der Zuwachs einen Anhaltspunkt. Auch hier profitiert die Variante ERTRAG in besonderem Maße. Einerseits setzt der Überlappungseffekt in dieser Variante früher ein, daß heißt die Umtriebszeitverkürzung durch Zusammenfallen der Endnutzungs- und Verjüngungsphase ist früher als in den anderen Varianten wirksam. Andererseits ziehen die gepflanzten Bäume Vorteile aus ihrer frühen Freistellung, in dem sie ihren Zuwachs erhöhen. Eine wichtige Frage wäre, in welchem Zeitraum sich die Investitionen in Pflanzung amortisieren. Zu erwarten ist, daß die Strategie ERTRAG wegen des Anbaus ertragsstarker Baumarten und den kürzeren Umtriebszeiten früher als die anderen Strategien, unter denen gepflanzt wird, von den Pflanzungen profitiert. Für den zukünftigen Einfluß der Pflanzungskosten ist entscheidend, inwieweit die angestrebte Baumartenmischung der Varianten LÖWE, PNV und ERTRAG nach dem Erreichen derselben durch natürliche Verjüngung unter den jeweiligen Nutzungsstrategien erhalten werden kann.

\subsubsection{Biologische Diversität}

Die Auswertung der Ergebnisse der Nachbarschaftsanalysen zeigt, daß sich die Durchmischung auf Landschaftsebene in bezug auf die Bestandestypenverteilung in allen vier Varianten erhöht. Der Durchmischungskoeffizient beträgt im Ausgangszustand 1,43 (siehe Kapitel 4.2.2) und erreicht damit $87 \%$ des unter Beibehaltung der Bestandesgrenzen maximal möglichen Werts (Definition siehe Kapitel 3.6.2). Am Ende des Simulationslaufs hat er in der Variante PNV 90\%, in den Varianten ERTRAG und PROZESS 91\% und in der Variante LÖWE 92\% des maximalen Werts erreicht.

Der Anstieg in allen Varianten ist einerseits dadurch zu erklären, daß vor Beginn der Simulation getroffene Waldumbaumaßnahmen Auswirkungen auf die Bestandesentwicklung in allen Varianten haben. Aus den Kiefernreinbeständen aus den Nachkriegsaufforstungen werden teilweise Mischbestände. So erhöht sich die landschaftliche Vielfalt, wenn verbliebene Reinbestände an Mischtypen grenzen oder sich verschiedene Mischungstypen aus den Reinbeständen entwickeln.

Der leicht höhere Durchmischungskoeffizient der Variante LÖWE ist durch die Vielfalt an verschiedenen Mischbestandestypen, die als Zielbestandestypen in dieser Variante angestrebt werden, zu erklären. Da in der Variante ERTRAG hauptsächlich auf Reinbestände hin gewirtschaftet wird, ist der Durchmischungskoeffizient dieser Variante etwas niedriger. In der Variante PNV werden größtenteils Drahtschmielen-Buchenwälder als potentiell natürliche Vegetation angesehen. Das führt dazu, daß der Bestandestyp in der Landschaft noch weniger variiert als in der Variante ERTRAG, in der verschiedene Reinbestandestypen (vor allem Douglasien- und Fichtenreinbestände) angestrebt werden. Die Entwicklung der Variante PROZESS zeigt, daß die landschaftliche Diversität auch in dieser Variante von den schon durchgeführten Umbaumaßnahmen profitiert. Da jedoch keine Zielbestandestypen angestrebt werden, ist zu erwarten, daß die landschaftliche Diversität in Zukunft sinken wird. Langfristig werden sich die auf den jeweiligen Standorten 
konkurrenzstärksten Baumarten (Kiefer, Fichte, Buche) durchsetzen, das an Bestandesgrenzen gebundene Mosaik der Bestandestypen wird sich auflösen.

Bei der Bewertung der biologischen Diversität anhand des Index-A ist ein Vergleich der Ergebnisse mit dem Status Quo (siehe Kapitel 4.2.2) problematisch, daher werden an dieser Stelle nur die Ergebnisse der Varianten untereinander verglichen. In den Varianten PROZESS und LÖWE sind die Werte des Index-A mit 0,98 bzw. 0,97 deutlich höher als in den Varianten ERTRAG $(0,89)$ und PNV $(0,86)$. Die Variante PROZESS schneidet in bezug auf den Index dadurch am besten $a b$, daß sie keine Pflanzungen vorsieht. Einerseits erhöht dies die vertikale Schichtung, da die Verjüngung ungleichaltrig ist und zu jedem Zeitpunkt Einwuchs möglich ist. Andererseits kommt es vielfach zum Einwuchs von schnellwachsenden Lichtbaumarten wie der Birke, die auf Flächen, auf denen gepflanzte Bäume stehen, seltener sind. Die großflächigen Pflanzungen in der Variante PNV verhindern das Einwachsen von Baumarten aus natürlicher Verjüngung, daher schneidet diese Variante in der hier abgebildeten Umbauphase am schlechtesten ab. In der Variante LÖWE ist die Pflanzfläche etwas kleiner als in der Variante ERTRAG, was ein Grund für einen im Vergleich zur Variante ERTRAG höheren Index-A ist. Der wichtigere Grund für das bessere Abschneiden der Variante LÖWE ist jedoch, daß dort Mischbaumarten explizit gefördert werden. Wenn die Umbauphase in den Varianten LÖWE, ERTRAG und PNV abgeschlossen ist, ist zu erwarten, daß sich der Index-A besonders in der mischbestandesfördernden Variante LÖWE noch erhöhen wird.

Von der Zielstärkennutzung würde man annehmen, daß sie eine höhere vertikale Struktur schafft als die schichtenweise Endnutzungsstrategie der Variante ERTRAG. Bei einem Vergleich der durchschnittlichen Anzahl der Schichten eines Bestandes kann dies für den Simulationszeitraum jedoch nicht bestätigt werden. Einerseits sind große Teile der Bestände noch nicht im Endnutzungsalter, andererseits profitieren alle Varianten von den bis zum Aufnahmezeitpunkt der Forsteinrichtung schon vollzogenen Umbaumaßnahmen gleichermaßen. Die vertikale Struktur wird durch die eingebrachte Verjüngungsschicht bereichert und durch das gleichzeitige Bestehen der Baumarten des Alt- und des Verjüngungsbestandes vorübergehend erhöht.

Die Entwicklung des anfallenden Totholzvolumens bestätigt die Beobachtungen aus den Beispielbeständen 1-3. Durch die zeitlich weiter auseinander liegenden Durchforstungsintervalle sterben in den Varianten PROZESS $(70,9 \mathrm{Vfm} / \mathrm{ha}$ pro vierzig Jahre) und ERTRAG (69,8Vfm/ha pro vierzig Jahre) mehr Bäume durch natürliche Mortalität ab als in den Varianten LÖWE $(57,8 \mathrm{Vfm} / \mathrm{ha})$ und PNV $(37,6 \mathrm{Vfm} / \mathrm{ha})$. Obwohl in den beiden Varianten LÖWE und PNV alle fünf Jahre in die Bestände eingegriffen wird, fällt in der Variante PNV weniger Totholz an als in der Variante LÖWE. Das liegt daran, daß die Vornutzungen in der Variante PNV so stark in die Bestände eingreifen, daß die natürliche Mortalität auf ein Minimum sinkt.

In den beiden Varianten LÖWE und PROZESS, die jeweils die Markierung eines Habitatbaums pro Hektar vorsehen, welcher der Nutzung dauerhaft entzogen wird, akkumuliert sich das durchschnittliche Habitatbaumvolumen pro Hektar auf 1,7 bzw. $2 \mathrm{Vfm} /$ ha. In der Variante PROZESS liegt dieser Wert ein wenig höher als in der Variante LÖWE. Durch die permanente Markierung von Z-Bäumen ist die 
Auswahlwahrscheinlichkeit für einen Habitatbaum in dieser Variante etwas geringer als in der Variante PNV. So werden in der Variante LÖWE durchschnittlich 0,9 Habitatbäume und in der Variante PROZESS ein Habitatbaum pro Hektar der Nutzung entzogen.

Um zu überprüfen, ob der Schutz von Habitatbäumen einen maßgeblichen Einfluß auf das Endnutzungsvolumen und auf die durch die Endnutzung erzielten Erträge hat, wurde ein Simulationslauf ohne die Auswahl von Habitatbäumen durchgeführt. Es konnten keine signifikanten Unterschiede festgestellt werden. Dies hängt aber sicherlich auch damit zusammen, daß ein Großteil der Habitatbäume die angestrebte Zielstärke noch nicht erreicht hat.

\subsubsection{Zielerreichung}

Keine der angestrebten Zieltypenverteilungen der Varianten (siehe Abbildung 4.3.4.a) konnte innerhalb des Untersuchungszeitraums erreicht werden (siehe Abbildung 4.3.4.b).
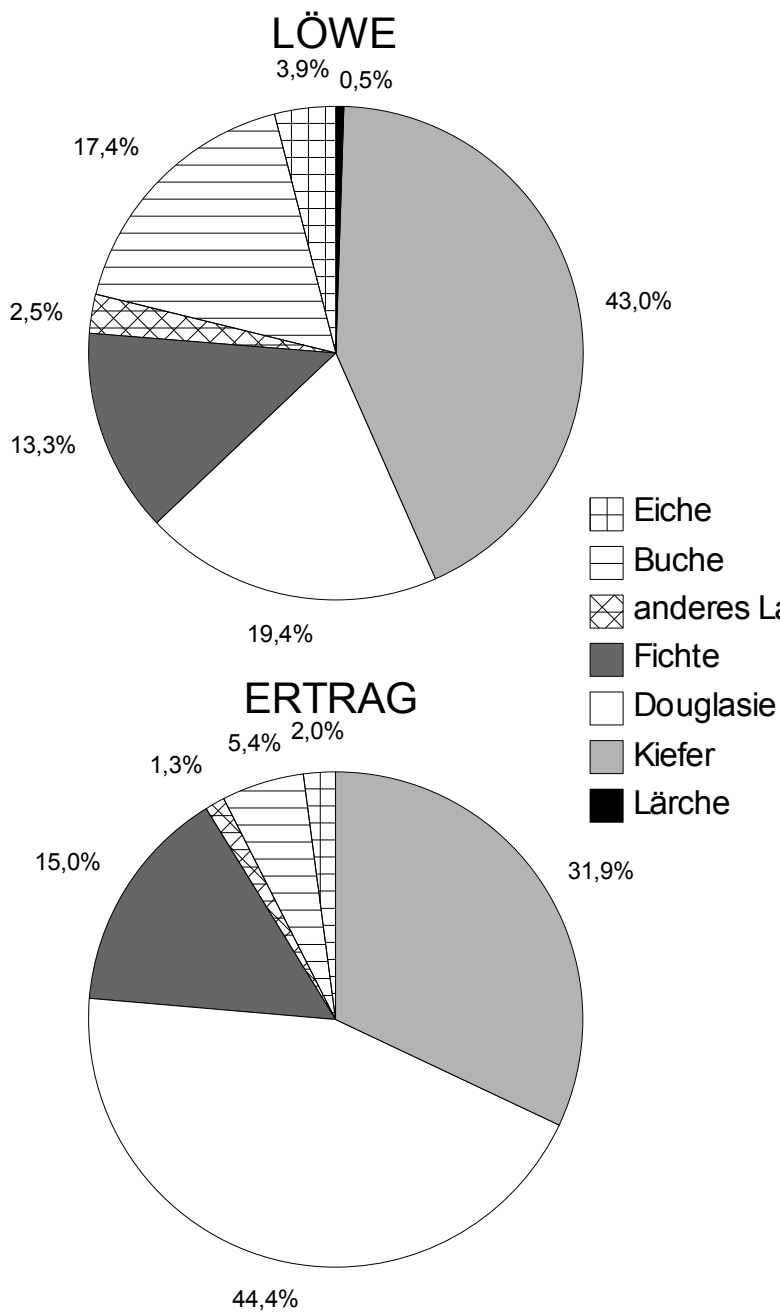

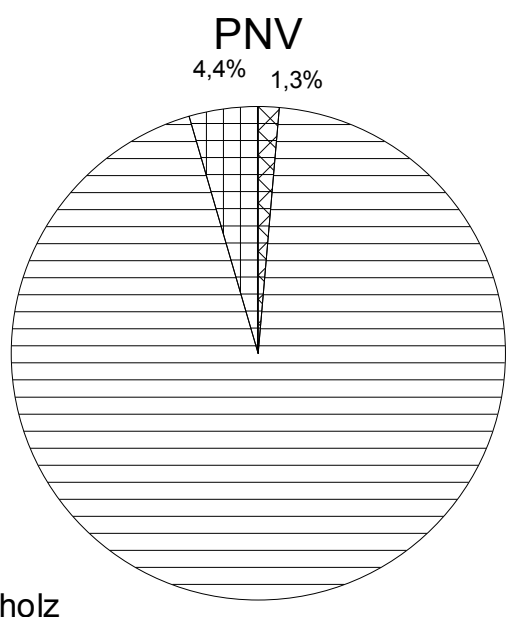

$94,3 \%$

PROZESS

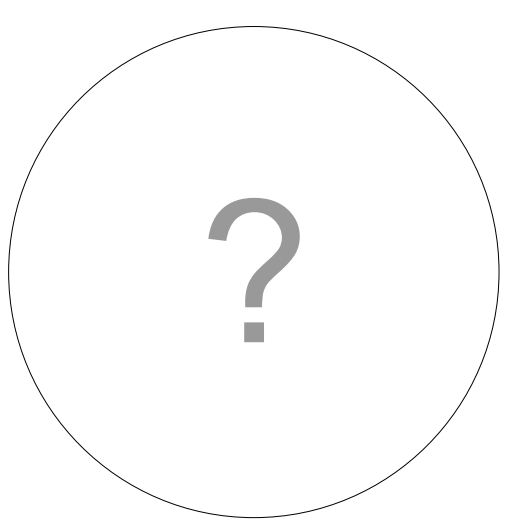

Abbildung 4.3.4.a: Verteilung der Zieltypenanteile der drei Varianten (LÖWE, PNV und ERTRAG) an der Fläche des Untersuchungsgebiets. Da in der Variante PROZESS keine keine Zieltypen angestrebt werden, können für diese Variante keine Anteile angegeben werden. 

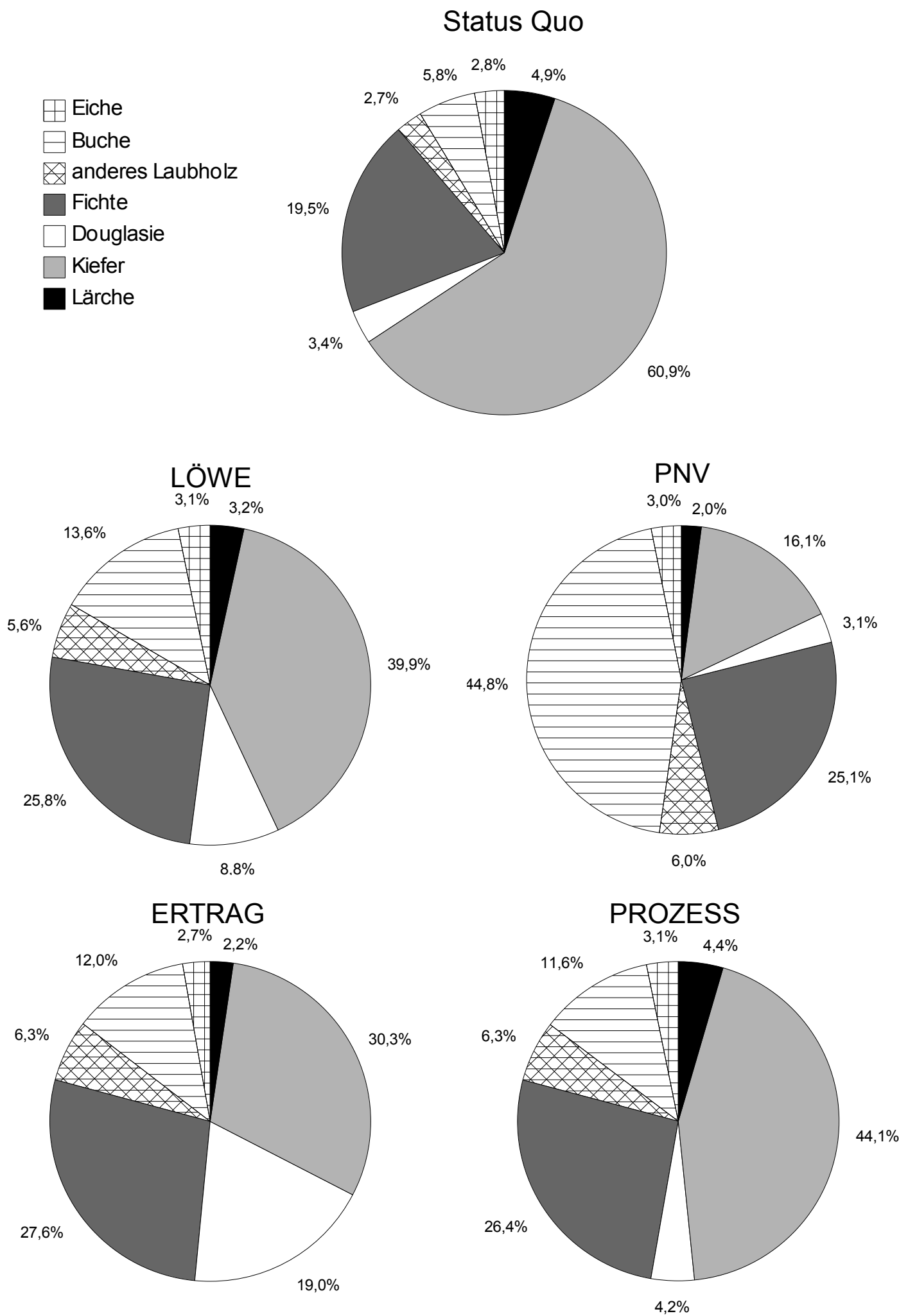

Abbildung 4.3.4.b: Verteilung der Bestandestypenanteile an der Fläche des Untersuchungsgebiets zum Anfang der Simulation (Status Quo) und am Ende der Simulationen der vier Varianten (LÖWE, PNV, ERTRAG und PROZESS) über einen vierzigjährigen Simulationszeitraum. 
Generell muß bei der Betrachtung der Varianten berücksichtigt werden, daß im Untersuchungsgebiet nur begrenzte Umbaumöglichkeiten innerhalb des Simulationszeitraums bestehen. In allen Varianten beeinflussen die zum Beginn der Simulation vorhandenen Pflanzungen die Bestandestypenverteilung. Auf ca. 39\% der Fläche besteht laut Forsteinrichtung zu Simulationsbeginn gesicherte Verjüngung. Eine Änderung der Baumartenzusammensetzung auf dieser Fläche kann sich nur auf Steuerung der Baumartenanteile beziehen. Ein Baumartenwechsel kann erst nach der Endnutzung des Bestandes, der sich aus dieser Verjüngung entwickelt, vollzogen werden. - also Jahrzehnte nach Ende des Simulationszeitraums. Hinzu kommt, daß in Beständen deren Verjüngung noch nicht festgelegt ist, Umbaumaßnahmen erst in - oder kurz vor - der Endnutzungsphase eingeleitet werden können. Die Zielerreichungsprozente zu Beginn und nach der Simulation der Varianten sind der Tabelle 4.3.a zu entnehmen.

Die Variante LÖWE hat den Vorteil, daß ihre Zielsetzungen bereits vor Beginn der Simulation über Jahrzehnte auf den größten Teil der untersuchten Bestände angewendet wurden. Das führt dazu, daß das Zielerreichungsprozent dieser Variante schon zu Beginn mit $48 \%$ höher ist als das der anderen Varianten. Innerhalb des Simulationszeitraums steigt das Zielerreichungsprozent der Variante auf 58\% an. Keine der anderen Varianten, in denen Zieltypen gesetzt werden (das heißt die Varianten PNV und ERTRAG), kommt ihrem angestrebten Mischungsverhältnis so nah. Mit der Annäherung an die angestrebten WETs sinkt in der Variante LÖWE der Kiefernanteil von 60,9\% auf 39,9\% der Kronenschirmflächensumme des Untersuchungsgebiets. Er liegt damit sogar unter dem in der Zieltypenverteilung vorgesehenen Wert von 43\%, da in den alten Kiefernbeständen größtenteils schon vor Simulationsbeginn Umbaumaßnahmen getätigt wurden. Vom Rückgang der Kiefer profitieren einerseits die Laubbaumarten, allen vorweg die Buche, deren Anteil von 5,8\% auf $13,6 \%$ steigt, andererseits aber auch ertragsstarke Baumarten wie die Fichte und die Douglasie. Der Fichtenanteil erhöht sich um ca. 6 Prozentpunkte auf $25,8 \%$ und der Douglasienanteil steigt von 3,4\% auf $8,8 \%$ an.

Am deutlichsten ist die Verschiebung der Flächenanteile hin zu mehr Laubwald im Rahmen der PNV-Variante zu beobachten. Hier wächst der Laubwaldanteil um fast das Fünffache von ca. 11\% im Status Quo auf 54\%. Mit dieser Variante wird somit eine Umkehr der bisherigen Anteile vom Nadelholz zum Laubholz erreicht. Besonders nimmt der Anteil der Buchen im Untersuchungsgebiet zu. Die Buchen-dominierten Bestandestypen verachtfachen in den vierzig Simulationsjahren ihren Flächenanteil im Rahmen dieser Variante. Auffällig ist im Zusammenhang mit dieser drastischen Umbauvariante, daß die fichtendominierten Waldflächen ebenfalls zunehmen (von 19,5\% auf $25,1 \%$ ). Dies ist auf die schon angesprochenen Pflanzungen, die für den Status Quo übernommen werden, zurückzuführen.

Deutlich geringer als bei der PNV-Variante fällt die Laubwaldvermehrung im Zuge der Managementstrategien ERTRAG und PROZESS aus. In beiden Fällen kann der Anstieg auf $21 \%$ voll auf die Umbaumaßnahmen der Vergangenheit zurückgeführt werden. Sehr deutlich zeigt sich das Prinzip der konsequenten Förderung der jeweils standortangepaßten, leistungsstärksten Baumart in der Bestandestypenzusammensetzung bei der Variante ERTRAG. Hier nimmt der Anteil der leistungsstarken Baumarten Douglasie, Buche und Fichte überdurchschnittlich zu: douglasiendominierte 
Bestandestypen steigen von 3,4\% auf 19\%, buchendominierte Bestandestypen von 5,8\% auf $12 \%$ und fichtendominierte Bestandestypen von $19,5 \%$ auf $27,6 \%$ der Gesamtwaldfläche.

Die Baumartenzusammensetzung, die sich bei der Simulation der Variante PROZESS ergibt, ist größtenteils auf die zu Beginn des Simulationszeitraums festgelegte Verjüngung zurückzuführen. Auf $7 \%$ der Fläche des Untersuchungsgebiets war zu Beginn der Simulation gesicherte Buchenverjüngung zu finden. In der Variante PROZESS steigt das Kronenschirmflächenprozent der Buche von 5,8\% auf $11,6 \%$ an. Gesicherte Fichtenverjüngung war zu Beginn der Simulation auf $12 \%$ der Fläche des Untersuchungsgebiets vertreten, der Fichten-Anteil stieg um ca. 7\%. Durch Douglasienpflanzungen, die auf $6 \%$ der Fläche zu Beginn der Simulation als gesichert bezeichnet wurden, konnte sich der Anteil dieser Baumart von 3,4\% auf 4,2\% erhöhen. Die Anteile dieser Baumarten vergrößerten sich auf Kosten der Kiefer. Ihr Anteil ging zwar nicht so stark zurück wie in den anderen Varianten, allerdings ist eine weitere Abnahme zu erwarten, da in der Variante PROZESS keine verjüngungsfördernden Maßnahmen durchgeführt werden. Ohne bodenvorbereitende Maßnahmen wird der Kiefernanteil in dieser Variante weiter sinken und der der Weichlaubhölzer stark zunehmen.

Die Kategorie „anderes Laubholz“ vergrößert sich in allen vier Varianten um mindestens das Zweifache. Anzunehmen ist, daß die Forsteinrichtungsdaten den Anteil von Weichlaubhölzern unterschätzen. Durch die Einwuchsfunktion kommt es zu einer realistischeren Einschätzung des Anteils dieser Arten, da die Funktionen anhand von Probekreisdaten kalibriert worden sind.

Für die zukünftige Entwicklung der Baumartenzusammensetzung ist entscheidend, inwieweit die szenarienabhängigen Zielbaumartenmischungen der Varianten LÖWE, PNV und ERTRAG durch natürliche Verjüngung unter den jeweiligen Nutzungsstrategien erhalten werden können.

\subsubsection{Vergleichende Übersicht}

Anhand der vergleichenden Übersicht (siehe Abbildung 4.3.5.a) werden die Auswertungsparameter der Varianten bereichsübergreifend gegenübergestellt. Auf die Darstellung der erntekostenfreien Holzerlöse für Vor- und Endnutzung wurde verzichtet, da diese in den Parameter "Holzproduktionswert“ direkt mit einfließen. Der Parameter „Pflanzfläche“ wird hier invertiert als „Pflanzungsfreie Fläche“ dargestellt, da dieser Wert im Zusammenhang mit dem Holzproduktionswert anschaulicher ist. Auf die Darstellung der Parameter „Durchmesserstruktur" und „Verteilung der Bestandestypen“ muß verzichtet werden, da sie nicht auf eine Zahl reduziert werden können, sondern erst in Form von Balken- oder Kreisdiagrammen einen Aussagewert erhalten.

Auffällig ist, daß die Variante PROZESS in allen Bereichen außer den Nutzungsmassen und dem Zuwachs im Vergleich zu den anderen Varianten die höchsten Werte für die Auswertungsparameter liefert. Es ist jedoch nicht zu erwarten, daß die Variante dieses Ergebnis in Zukunft halten kann. 


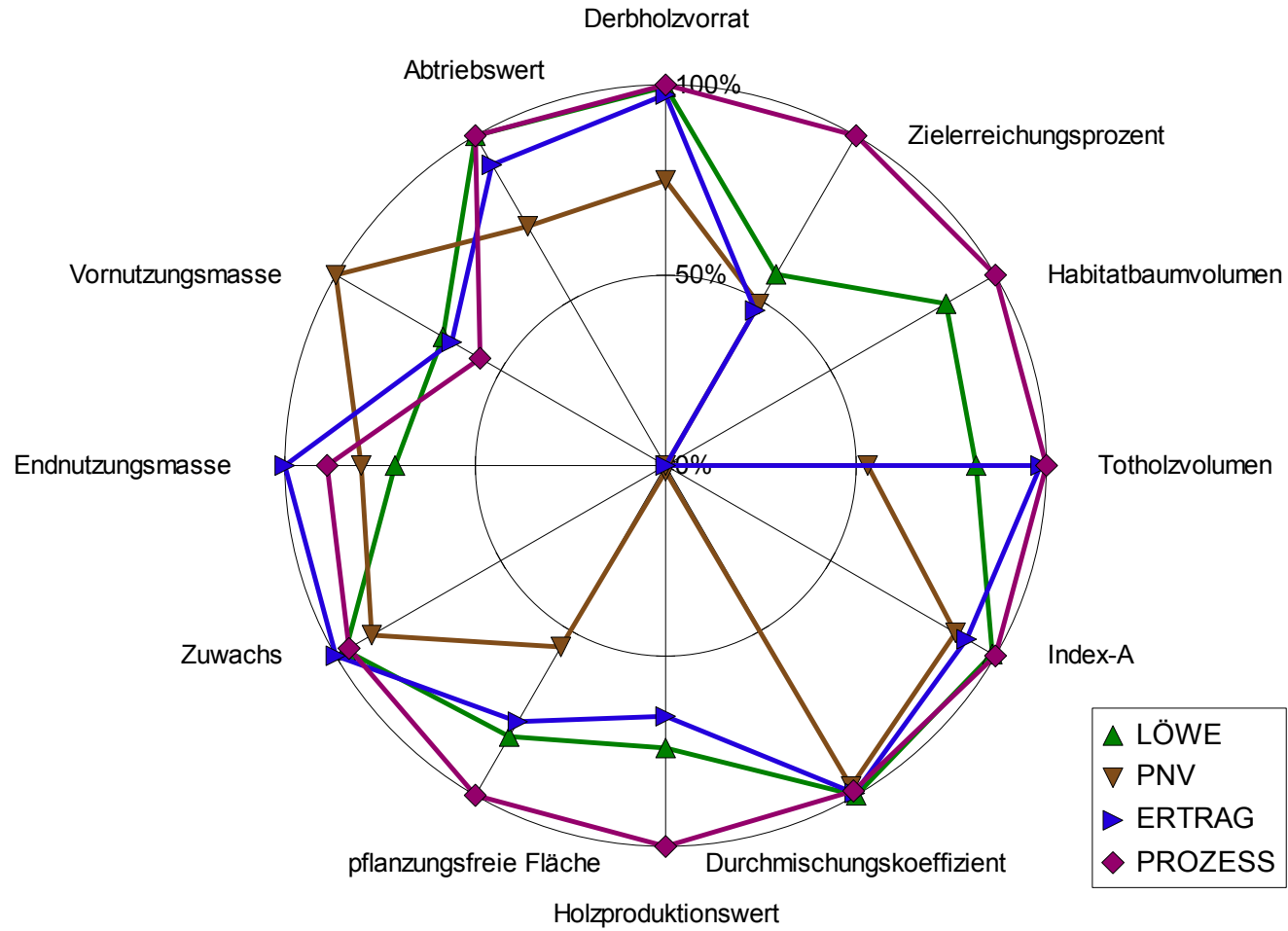

Abbildung 4.3.5.a: Vergleichende Übersicht ausgewählter Auswertungsparameter des Untersuchungsgebiets nach der vierzigjährigen Simulation der Varianten LÖWE, PNV, ERTRAG und PROZESS. Die Achsen der einzelnen Parameter bezeichnen mit $100 \%$ den jeweils höchsten vorkommenden Wert der Simulationsläufe. Die Datenpunkte geben an, wieviel Prozent dieses Werts in der jeweiligen Variante erreicht wurden.

Nur der hohe Totholzanteil wird auch in Zukunft bestehen bleiben, da er auf die unzureichenden Durchforstungsmaßnahmen und den dadurch hohen Derbholzvorrat zurückzuführen ist.

Die Entwicklung der Bestände innerhalb der Simulationsperiode profitiert in bezug auf die anderen Parameter von den Pflanzungen, die zu Beginn des Simulationszeitraums schon getätigt worden waren. Für die Zukunft ist zu erwarten, daß durch Vergrasung in Kiefernaltbeständen und durch das verstärkte Aufkommen von Weichlaubhölzern die Bestände in ihrer Wertentwicklung und der Entwicklung ihrer Diversität nicht mit den anderen drei Varianten mithalten können. Bei der monetären Betrachtung ist zu berücksichtigen, daß innerhalb des Simulationszeitraums noch viele Bäume genutzt werden, deren Qualität von Pflegemaßnahmen des vorherigen Waldbaukonzepts (meist LÖWE oder ein an LÖWE angelehntes Konzept) bestimmt wurde. Im Vergleich zu den anderen Varianten wird sich die Qualität der Bestände wegen der diskontinuierlichen Pflegemaßnahmen weiter verschlechtern. Durch das Zusammenspiel von Kosteneinsparung durch Verzicht auf Pflanzungen und dem Profitieren von waldbaulichen Maßnahmen der Vergangenheit schneidet die Variante auf vielen Gebieten innerhalb des Simulationszeitraums besser ab, als sie es in Zukunft können wird. 
Die Variante PNV schneidet in vielen Punkten schlechter ab als die anderen Szenarien. Als radikales Umbaukonzept weist sie die höchsten Vornutzungsmassen auf, wodurch sie den Derbholzvorrat des verbleibenden Bestandes weit absenkt. Natürliche Mortalität findet nur begrenzt statt (siehe Totholzvolumen), da der Konkurrenzdruck unter den Bäumen durch die starken Eingriffe herabgesetzt wird. Die biologische Diversität der Variante leidet insgesamt unter der starken Beschränkung auf die Förderung der Baumart Buche. Betrachtet man die Differenz der Baumartenzusammensetzung zwischen Beginn und Endzeitpunkt der Simulation oder auch die Veränderung des Zielerreichungsprozents, so kann man die Strategie durchaus als erfolgreich bezeichnen. Allerdings ist mit dem Umbau ein sehr hoher Pflanzaufwand (siehe pflanzungsfreie Fläche) verbunden, so daß das Vorgehen der Variante unter betriebswirtschaftlichen Gesichtspunkten kaum gerechtfertigt werden kann (siehe Holzproduktionswert). Ist die Umbauphase erst einmal abgeschlossen, wird die Variante durchaus mit den anderen Varianten vergleichbar abschneiden können, wie die Betrachtung des Holzproduktionswerts ohne Pflanzungskosten zeigt (siehe Kapitel 4.3.2). Problematisch ist jedoch die Einschränkung der Produktpalette durch die weitgehende Einschränkung auf die Baumart Buche in dieser Variante. Ein breiteres Sortiment ermöglicht ein flexibleres Eingehen auf Marktbedürfnisse und fördert dadurch die Ertragserwartung.

Das Kriterium der biologischen Diversität blieb beim Entwurf der Variante ERTRAG unberücksichtigt, dies spiegelt sich in den Parametern Index-A, Habitatbaumvolumen und Durchmischungskoeffizient wider. Allerdings schneidet die Variante im Hinblick auf die biologische Diversität besser ab als die Variante PNV. Die Stärke der Variante ERTRAG liegt in der Erfüllung der Produktionsfunktion. Die Variante ERTRAG setzt sich durch hohe Nutzungsmassen und einen hohen Zuwachs von den anderen drei Varianten ab. Wegen der begrenzten Simulationszeit und der Nicht-Berücksichtigung einiger betriebswirtschaftlicher Faktoren kommt das Potential der Variante ERTRAG jedoch nur bedingt zum Ausdruck. Wie in Kapitel 4.3.2 schon diskutiert wurde, werden Vorteile durch Auflagendegression und Reduktion des Organisationsaufwands nicht berücksichtigt. Auch die gegebene Durchmesserstruktur des Untersuchungsgebiets (siehe Kapitel 4.3.1) läßt Vorteile der Endnutzungsstrategie nicht deutlich werden, da ein Großteil der Bestände erst in den Jahrzehnten nach der Simulation in die Endnutzungsphase übergeht. In Zukunft wird die Variante durch den Umbau auf ertragsstarke Baumarten stark profitieren können, der Umbau des Untersuchungsgebiets konnte im Simulationszeitraum weit vorangetrieben werden. In den Beständen kann man hohe Zuwächse und eine positive Wertentwicklung erwarten. Durch niedrigere Zielstärken und zeitlich begrenzte Endnutzungszeiträume verkürzen sich die Umtriebszeiten. Die getätigten Pflanzungen amortisieren sich bei kürzeren Umtriebszeiten und dem Anbau ertragsstarker Baumarten schneller, als dies in den anderen beiden pflanzenden Varianten der Fall ist. Zu beachten ist allerdings auch, daß der erzielte Erlös in der Endnutzung stark vom Verlauf der Erlösfunktion abhängt. Die angestrebte Zielstärke muß stets an den aktuellen Holzpreis angepaßt werden.

Die Variante LÖWE zeichnet sich durch ihre Ausgewogenheit aus, sie weist weniger Extremwerte als die anderen Varianten auf. Mit dem Anstreben von Mischbeständen gelingt es ihr, die biologische Diversität zu fördern. Sowohl der Durchmischungskoeffizient als auch der Index-A dieser Variante werden von der Strategie positiv beeinflußt. Die 
Auswahl von Habitatbäumen und der Schutz von Minderheiten fördert die Diversität der Bestände weiterhin. Auch in bezug auf forstliche Ressourcen und die Produktionsfunktion ist die Entwicklung des Untersuchungsgebiets unter dieser Strategie positiv zu beurteilen. Die Variante LÖWE schafft es, durch ihre mäßigen Durchforstungseingriffe die Bestände bei einem relativ hohen Derbholzvorrat wertsteigernd zu differenzieren. Sie wird in Zukunft weiter von den teilweise schon vor dem Startzeitpunkt der Simulation eingeleiteten Pflanzungen profitieren. Sie fördert standortsgerecht ertragsstarke Baumarten, ohne auf Mischbestände zu verzichten. Damit und durch ihre Strategie der Zielstärkennutzung kann sie auf sich ändernde Marktbedürfnisse flexibel reagieren.

\subsubsection{Fazit}

Zu beachten ist in allen vier Varianten, daß die Entwicklung im Simulationszeitraum nicht nur durch das jeweilige Konzept beeinflußt wird. Qualität, Struktur und Baumartenzusammensetzung der Bestände des Untersuchungsgebiets sind auch nach vierzig Jahren noch von dem bislang verfolgten Waldbaukonzept LÖWE geprägt. Besonders bei der betriebswirtschaftlichen Bewertung wird dies deutlich. Die gewählten Maßnahmen führen nicht zwangsläufig innerhalb von vierzig Jahren zu den bezweckten Auswirkungen. Die Umstellung des waldbaulichen Managementkonzepts ist in den Varianten ERTRAG und PNV mit hohen Kosten verbunden. Auch in der Variante LÖWE werden mit dem Baumartenwechsel erhebliche Investitionen getätigt. In einem Planungshorizont von vierzig Jahren können diese sich noch nicht amortisieren.

Auch die waldbauliche Ausgangssituation hat auf den Erfolg der Strategien Einfluß. Der Vorratsaufbau, die Nutzungsmöglichkeiten, die biologische Diversität und die Möglichkeit zur Steuerung der Baumartenzusammensetzung hängen von ihr ab. Man muß das Untersuchungsgebiet vor dem Hintergrund betrachten, daß sich die Bestände im Vorratsaufbau befinden und auf vielen Flächen ein Baumartenwechsel vorbereitet wurde.

Trotz dieser Einschränkungen haben die umgesetzten Waldbaustrategien deutliche Auswirkungen auf die Bestände des Untersuchungsgebiets. In allen Bereichen (,forstliche Ressourcen“, „Produktionsfunktion“, „biologische Diversität“ und „Zielerreichung“) können szenarienabhängige Entwicklungen belegt werden, die mit der szenarienabhängigen Maßnahmenwahl und den individuell gesetzten Rahmenwerten begründet werden können. So steigt zum Beispiel der Derbholzvorrat in einer eingreifenden Variante weniger (PNV) als in einer schwach eingreifenden Variante (PROZESS). In der Durchmesserstruktur und der Endnutzungsmasse zeigen sich in der Variante ERTRAG deutlich die Auswirkungen der früh einsetzenden Endnutzungsmaßnahmen. Die Baumartenzusammensetzung kann in den drei Varianten LÖWE, PNV und ERTRAG durch die im Untersuchungszeitraum getätigten Pflanzungen verändert werden. Der Baumartenwechsel wirkt sich auch auf die Diversitätskriterien aus.

Insgesamt kann der Ansatz als erfolgreich bezeichnet werden, waldbauliche Strategien in Maßnahmenelemente zu zerlegen, die in variantenspezifischen Maßnahmenketten zusammengesetzt und durch szenarienabhängige Rahmenwerte gesteuert werden. Es stellt sich jedoch die Frage, wie die Auswertung und Darstellung der Ergebnisse optimiert 
werden kann, um dem Nutzer auch die langfristigen Vor- und Nachteile einer Strategie transparent zu machen.

\section{Schlußbetrachtung und Ausblick}

Bei der Beurteilung eines Modells sollte immer der Gesichtspunkt berücksichtigt werden, welche Grenzen ihm gesetzt sind. Auf diese Grenzen wird im Unterkapitel 5.1 eingegangen. Insgesamt sind alle Simulationsergebnisse nachvollziehbar, und es bilden sich strategiebedingte Charakteristika heraus. Allerdings gibt es einige Ansatzpunkte, von denen ausgehend die Aussagequalität der Simulation entscheidend verbessert werden könnte (Kapitel 5.2). Weitere Anwendungsgebiete und Implementierungsmöglichkeiten werden in Kapitel 5.3 dargestellt.

\subsection{Grenzen des Systems}

Das Wesen eines Modells liegt in der Abstraktion von der Wirklichkeit. Abstraktion ermöglicht es, dem Betrachter wesentliche Aspekte sichtbar und vergleichbar zu machen. Auch im Rahmen dieser Arbeit wurden vereinfachende Annahmen getroffen, die auf der einen Seite den Umfang der berücksichtigten Faktoren beschränken, auf der anderen Seite aber eine deutlichere Abbildung berücksichtigter Faktoren ermöglichen. Die überschaubare Reduktion der Komplexität ist auf der Ebene der Rahmenbedingungen und der Ebene des Modells zu betrachten.

Ein Kernanliegen ist, die Transparenz über Einflußmöglichkeiten von Managementstrategien auf die Entwicklung von Beständen zu erhöhen. Somit liegt das Augenmerk auf dem Potential, das hinter der Summe von Einzelentscheidungen des Waldbesitzers steht. Effekte, die außerhalb des menschlichen Einflußbereiches stehen oder auf die Einzelpersonen keinen nennenswerten Einfluß haben, bleiben unberücksichtigt. Zu diesen Effekten gehören langanhaltende extreme Wetterlagen (wie zum Beispiel extreme Sommertrockenheit), Klimaveränderungen, der Einfluß von Stoffeinträgen aus der Atmosphäre oder die Folgen von Veränderungen des Grundwasserspiegels. Auf die Abbildung von Wildschäden wird aus Gründen der Komplexität ebenfalls verzichtet. Auch Kalamitäten wie Windwurf, Brände oder Insektenmassenvermehrung bleiben unberücksichtigt. Das Risiko solcher Ereignisse ist zwar von waldbaulichen Maßnahmen abhängig, jedoch stehen Häufigkeit und Ausmaß eines Schadereignisses nicht im direkten Zusammenhang mit dem waldbaulichen Handeln. Es bleiben Zufallskomponenten, da der Waldbesitzer weder auf extreme Wetterlagen noch auf das Handeln der anderen Waldbesitzer in seiner Umgebung direkten Einfluß nehmen kann. Durch die getroffenen Vereinfachungen werden konstante Rahmenbedingungen geschaffen, die in der Realität so nicht existieren. Der Gesamtvorrat wird mit Verlängerung des Simulationszeitraums zunehmend überschätzt, der Einfluß von Schadereignissen auf die Baumartenmischung bleibt unberücksichtigt.

Aber nicht nur die Rahmenbedingungen für Waldwachstum, sondern auch das Waldwachstumsmodell selbst muß abstrahiert werden. Die Modellierung der Waldentwicklung beruht auf der Simulation des Wachstums einzelner Bäume. Trotz der 
Beschränkung auf die Simulation von Bäumen mit einem BHD, der über 7cm liegt, ergäbe sich für das Untersuchungsgebiet ein enormer rechnerischer Aufwand. Betrachtet man beispielsweise den Status quo, müßten im Zuge der Wachstumsmodellierung knapp elf Millionen Bäume berücksichtigt werden, was gegenwärtig ressourcentechnisch nicht zu leisten ist. Zur Verringerung des Rechenaufwands wurden deshalb alle zu analysierenden Waldbestände des ca. 12.000 Hektar großen Untersuchungsgebiets auf eine ideelle Modellfläche von 0,25 Hektar reduziert. Insgesamt ergibt sich somit eine simulierte Fläche von 1326 Hektar, womit sich die Modellierungsdauer für eine Variante in einem noch akzeptablen Rahmen von zwei bis drei Tagen einpendelt.

Eine weitere Grenze des Systems liegt auch darin, daß jeder Bestand isoliert von den inn umgebenden Beständen betrachtet wird. Waldaußenränder, aber auch Abhängigkeiten der Bestände untereinander (zum Beispiel Verjüngungsmöglichkeiten) bleiben unberücksichtigt.

Das Ziel dieser Arbeit, einen entscheidungsrelevanten Zeithorizont abzubilden wurde erreicht. Die zukünftige Waldentwicklung kann durch die Begrenzung des Simulationszeitraums auf vierzig Jahre jedoch nur ansatzweise aufgezeigt werden. Es werden - gerade in Phasen ausgedehnter Waldumbauprogramme - nur Tendenzen sichtbar, die erst durch eine verläßliche Modellierung über einen sehr langen Zeitraum hinweg verifiziert werden könnten. Vor dem Hintergrund der langfristigen forstlichen Produktionszeiträume wäre eine Abbildung einer gesamten Umtriebszeit wünschenswert. Ob solche Informationen betriebliche Entscheidungen beeinflussen würden ist jedoch ungewiß.

Mit der Prognosedauer steigt auch die Unsicherheit der monetären Bewertung. Die künftig geltenden Kosten und Erlöse können immer nur aus der zurückliegenden Preisentwicklung heraus geschätzt werden. Schon innerhalb des Simulationszeitraums bedeutet diese Unsicherheit eine Einschränkung für die Verwendung der Ergebnisse. Da der betriebswirtschaftliche Erfolg stark von den sich ändernden Marktanforderungen abhängt ist eine Prognose auf diesem Gebiet eine Herausforderung.

\subsection{Weiterer Forschungsbedarf, Verbesserungen}

Das Unterkapitel 5.2.1 beschäftigt sich mit der Frage, inwieweit eine Kalibrierung der Eingangsparameter die Simulationsqualität verbessern kann. Im Unterkapitel 5.2.2 werden Vertiefungsmöglichkeiten, die den Ablauf der Simulation betreffen, aufgezeigt. Abschließend werden mögliche Modifikationen der Ausgabeparameter im Unterkapitel 5.2.3 diskutiert.

\subsubsection{Anmerkungen zum Bestandesaufbau}

Neben den betrachteten Rahmenbedingungen, dem Waldwachstumsmodell selbst und der Auswahl der Auswertungsparameter beeinflussen die Eingangsdaten die Aussagekraft der gesamten Simulation entscheidend. Der Aufbau der Waldbestände zu Beginn der Modellierung basiert unter anderem auf den Daten der Forsteinrichtung, die - wenn nötig durch Datenergänzungsroutinen vervollständigt werden. Hierzu werden Ertragstafelwerte 
herangezogen, welche wiederum selbst auf Modellen basieren. Die realen Bestandesdurchmesser werden durch die Ertragstafeln systematisch unterschätzt, was dann in der Waldwachstumssimulation mit TreeGrOSS zwangsläufig seinen Niederschlag findet. Wie in Kapitel 4.2.4 angemerkt, wird der Dg der Bestände beim Aufbau der Simulationsbestände im Status Quo unterschätzt. Funktionen zur Kalibrierung des Dg der Ertragstafeln sind in der Veröffentlichung von Wollborn und Böckmann (WoLLBORN UND Böckmann 1998) zu finden, sie können eine sinnvolle Ergänzung der Datenergänzungsroutinen darstellen.

Eine weitere Frage bei der Verwendung von Forsteinrichtungsdaten ist, wie mit der tendenziellen Unterschätzung von seltenen Baumarten durch die Forsteinrichtung umgegangen werden kann. Denkbar wäre eine Baumartenergänzung der Bestände, die auf Erfahrungswerten aus Einzelbaumaufnahmeverfahren fußt.

Bei der modellhaften Generierung stellen Bestände, in denen viele verschiedene Baumarten in vielen verschiedenen Altersstufen vorkommen, ein Problem dar. (Im Untersuchungsgebiet gibt es in seltenen Fällen Bestände, in denen über zehn Arten aufgenommen wurden). Jede vorkommende Baumart eines Alters (entspricht einer Zeile der Forsteinrichtungsdaten) wird durch mindestens einen Baum im Modellbestand repräsentiert. Die Größe der einzelnen Modellflächen wird jedoch nicht in Abhängigkeit der Baumartenanzahl oder der Diversität des Bestandes festgelegt. So können sich die Baumartenanteile im Vergleich $\mathrm{zu}$ den realen Bestandesflächen verschieben. Minderheitenanteile und die Dichte des Bestandes können überschätzt werden.

Eine weitere Verbesserungsmöglichkeit im Rahmen des Bestandesaufbaus liegt in der Zufallsverteilung der Einzelbäume auf der Modellfläche. Die Forsteinrichtungsdaten weisen gleichartige und räumlich voneinander getrennte Bestandesteile als Hilfsfläche aus, deren geringe Größe nicht das Ausscheiden einer Unterabteilung zulassen. Im derzeitigen Bestandesaufbau wird die räumliche Trennung dieser Bestandesteile nicht umgesetzt. Bäume, die auf Hilfsflächen stehen, könnten geklumpt in den Bestand eingebracht werden, anstatt gleichmäßig über die Fläche verteilt zu werden.

\subsubsection{Ablauf}

Wie im Kapitel 5.1 beschrieben, wäre eine Ausdehnung des Simulationszeitraums eine wertvolle Erweiterung des Programms. Langfristiges Ziel könnte für die Entwicklung des Waldwachstumssimulators die Darstellung eines "Steady States“ sein, so daß eine Gesamtbewertung der Waldbaustrategien möglich ist. Allerdings stellt sich die Frage, ob die Abstraktionsebene, die ein Einzelbaumwachstumsmodell ansetzt, für eine langfristige Betrachtung das Mittel der Wahl sein kann. Forschungsbedarf besteht besonders bei der Einwuchssteuerung. Verjüngungs- und Pflanzungsroutinen müßten konkretisiert werden. Denkbar ist zum Beispiel die Berücksichtigung der Entwicklung der Verjüngung aus Pflanzung oder auch das Einbeziehen von Gründen, die das Ankommen von Verjüngung verhindern (wie Vergrasung). Weitere Verbesserungspotentiale, die eine langfristigere Betrachtung ermöglichen könnten, liegen in den Mortalitätsfunktionen (zum Beispiel für Habitatbäume) und der Berücksichtigung von Kalamitäten oder dem Kalamitätsrisiko. 
Außerdem wird eine Einschätzung der Bonität von Baumarten benötigt, die zu Beginn der Simulation noch nicht im Bestand vorgekommen sind.

Gerade im Hinblick auf die monetäre Bewertung der Varianten könnte das Modell verbessert werden, wenn man den räumlichen Bezug der Bestände untereinander berücksichtigte. Auf diese Weise wäre es zum Beispiel möglich, Entnahmevolumina forstlicher Eingriffe von benachbarten Beständen aufzuaddieren. So könnten Kostendegressionseffekte berücksichtigt werden, die zum Beispiel durch Maschineneinsatz und geringeren organisatorischen Aufwand entstehen.

Ein weiterer Ansatzpunkt für die Verbesserung des Modells ist die flexiblere Gestaltung von Eingriffsintervallen. Erfüllt ein Bestand zum Beispiel zu Beginn des Simulationszeitraums die Kriterien für die Durchführung einer waldbaulichen Maßnahme nicht, so wird erst nach Ablauf des Eingriffsintervalls (fünf bzw. zehn Jahren), die Notwendigkeit einer Maßnahme untersucht. Denkbar ist es, in jedem darauffolgenden Jahr erneut die Möglichkeit eines Eingriffs zu überprüfen. Das Eingriffsintervall würde dann als minimaler Zeitraum bis zum nächsten Eingriff und nicht als starres Zeitschema verstanden werden.

Natürlich bieten auch die Waldbaustrategien selbst Ausbaumöglichkeiten. Die Auswahl der Maßnahmenelemente könnte zum Beispiel vergrößert werden. An dieser Stelle wäre eine Methode, die grundflächensteuernde Eingriffe ermöglicht oder eine Niederdurchforstungsroutine denkbar. Für einen potentiellen Anwender ist es sinnvoll, wenn er auf die Rahmenwerte und auf die Maßnahmenkette (siehe Kapitel 3.5) direkten Zugriff hat und so den Ablauf der Waldbaustrategien nach seinen Vorstellungen gestalten kann.

Neben inhaltlichen Änderungen ist eine Verkürzung der Laufzeit der Simulation anzustreben. Erst wenn der Vergleich von Waldbaustrategien in einem für den Benutzer überschaubaren Zeitrahmen abläuft, kann ein solcher Simulator für den Praktiker interessant sein. Potentiale für eine Optimierung des Programmcodes bestehen zum Beispiel bei der Baumdatenhaltung. Statt der Verwendung von Arrays kann mit Hashtables gearbeitet werden. Außerdem ist zu prüfen, ob die Datenausgabe weiter optimiert werden kann. Ein Doppelter Aufruf von Methoden sollte vermieden werden, und darüber hinaus sind Möglichkeiten zum verstärkten Einsatz statischer Methoden zu prüfen.

\subsubsection{Ausgabeparameter}

Der Aussagewert der Modellergebnisse hängt auch von der Auswahl der Ausgabeparameter und der hinterlegten Funktionen zur Berechnung dieser Parameter ab. In diesem Zusammenhang bietet die Änderung der Berechnung des erntekostenfreien Abtriebswerts noch Verbesserungspotential auf dem Gebiet der forstlichen Ressourcen. Die in dieser Arbeit verwendeten Erlösfunktionen berücksichtigen nur die konventionelle Nutzung des Bestandes; das heißt sie gehen davon aus, daß das Holz als Stamm oder in Abschnitten nach motormanueller oder harvestergestützter Aufarbeitung verkauft wird. Bis zu einem gewissen BHD wird eine defizitäre Nutzung von Bäumen unterstellt, der Abtriebswert kann somit negative Werte annehmen. Zieht man jedoch auch die 
Verwertung von schwachem Holz zur Hackschnitzelgewinnung oder den Verkauf an Selbstwerber in Betracht, so können die Erlösfunktionen auch bei niedrigen BHDs im positiven Bereich liegen. Gerade in mehrschichtigen Beständen würde der Abtriebswert an Aussagekraft gewinnen. Ein Altbestand mit einer Unterschicht wäre dann ebensoviel (oder leicht mehr) wert wie ein Altbestand ohne Unterschicht. Mit den verwendeten Funktionen ist dem nicht so: In einem zweischichtigen Bestand kann die Unterschicht oft nur einen negativen erntekostenfreien Holzerlös erzielen, was den Gesamtwert des Bestandes in der Summe schmälert.

Für die betriebswirtschaftliche Bewertung im Rahmen der Produktionsfunktion würde außerdem die Einbindung einiger zusätzlicher Faktoren (zum Beispiel Planprozeßkosten) eine höhere Aussagekraft ermöglichen. Im Rahmen des BMBF-Projekts „FOREST“ (Fkz. 01 LM 0207) wurden Kosten für Wertästung und Jungwuchspflege mit in die Bewertung einbezogen und die in Tabelle 5.2.3.a angegebenen Korrekturfaktoren verwendet.

Eine Berücksichtigung dieser Faktoren beeinflußt die monetäre Bewertung stark, sie führt zum Beispiel bei der Betrachtung des Holzproduktionswerts zu einem veränderten Verhältnis der Varianten untereinander (besonders profitiert die Variante ERTRAG). Zu einer ganzheitlichen betriebswirtschaftlichen Bewertung fehlen allerdings immer noch die Kosten für Holzanweisen, die Holzaufnahme, Forstschutzkosten, Wegebaukosten und Kosten im Zusammenhang mit Sozialfunktionen. Eine Verknüpfung des vorgestellten Waldwachstumsmodells mit einem ausgereiften Bewertungsmodell würde eine umfassendere Bewertung des Betriebsergebnisses erlauben.

Tabelle 5.2.3.a: Kostenzuschläge und Kostenabschläge im Bereich der Holzernte für die Strategien LÖWE, PNV, ERTRAG und PROZESS.

\begin{tabular}{|c|c|c|c|c|c|c|}
\hline \multirow[t]{2}{*}{ Einflußfaktor } & \multirow{2}{*}{$\begin{array}{l}\text { Kostenzuschläge/ } \\
\text { Kostenabschläge } \\
(\boldsymbol{E} / \mathrm{Fm})\end{array}$} & \multicolumn{4}{|c|}{ Waldumbaustrategie } & \multirow[t]{2}{*}{ Literaturquelle } \\
\hline & & อิ: & $\gtreqless$ & 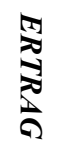 & 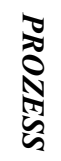 & \\
\hline Hiebsart & Kahlhiebe: $-0,5 €$ & & & $x$ & & KoEPKE 1999 \\
\hline $\begin{array}{l}\text { Maßnahmen je Jahrzehnt } \\
\text { (n/10 Jahre) }\end{array}$ & $2 / 10$ Jahre: $+0,5 €$ & $x$ & $x$ & & & HANEWINKEL 1998 \\
\hline $\begin{array}{l}\text { Größse der Hiebsfläche } \\
\text { (ha) }\end{array}$ & Über 4 ha: $-1,0 €$ & $x$ & $x$ & $x$ & $\mathrm{x}$ & KoEPKE 1999 \\
\hline $\begin{array}{l}\text { Erntemenge insgesamt } \\
(\mathrm{Fm})\end{array}$ & $\begin{array}{l}\text { Unter 500Fm: }+3,5 € \\
\text { Über } 2500 \mathrm{Fm}:-1,0 €\end{array}$ & $\mathrm{x}$ & $x$ & $x$ & $\mathrm{x}$ & PAUSCH 2005 \\
\hline
\end{tabular}

Nicht nur die betriebswirtschaftliche Bewertung bietet Verbesserungspotentiale. Auch bei der Betrachtung der biologischen Diversität könnten die Auswertungsparameter an Aussagekraft gewinnen. Für die Betrachtung der Durchmischung auf Landschaftsebene bietet sich zum Beispiel die Funktion „Contrast“ des Programms „Fragstats“ an. In dieser Arbeit wird lediglich zwischen verschiedenen Bestandestypen unterschieden. Jeder Bestandestypenwechsel fließt mit gleichem Gewicht in die Auswertung ein. Mit dieser Funktion hat man die Möglichkeit, eine Rangfolge in die unterschiedenen Klassen zu bringen. Den Wechsel zwischen einem Buchenreinbestand (Bestandestyp 20) und einem 
Buchen-/Bergahornbestand (Bestandestyp 22) kann man demnach geringer gewichten als den Wechsel zwischen einem Buchenreinbestand (Bestandestyp 20) und einem Kiefernreinbestand (Bestandestyp 70). Auch die Einbeziehung von unterschiedlichen Altersstufen kann sinnvoll sein. In diesem Zusammenhang wäre zum Beispiel eine Unterscheidung zwischen Buchenstangenholzbeständen und Buchenaltholzbeständen denkbar.

Bei einem Vergleich der in der Simulation berechneten Totholzmengen mit Informationen aus anderen Quellen muß die jeweilige BHD-Schwelle, mit der das Totholz aufgenommen wird, berücksichtigt werden. Simulationsbedingt liegt die Schwelle für das registrierte Totholz in der Simulation bei ca. $7 \mathrm{~cm}$. Bei einer Variation der betrachteten BHD-Schwelle ist das Einbeziehen von Ausgleichsfunktionen denkbar. Dies würde einen Vergleich zwischen Daten mit verschiedenen Aufnahmeschwellen ermöglichen. Solche Funktionen sind teilweise in der Literatur zu finden (zum Beispiel Christensen et al. 2005). Außerdem ist es möglich, im Wald verbliebenes X-Holz zu dem Totholzaufkommen zu addieren. Dadurch würde der direkte Bezug zwischen dem Anfall der Totholzmenge und der Durchforstungsintensität schwächer werden.

\subsection{Anwendungsgebiete und Implementierungsmöglichkeiten}

Es gibt viele Anwendungsgebiete von Decision Support Systemen im Forstbereich, allerdings liegen derzeit keine vollständig entwickelten Systeme zur Anwendung vor (voN TEUfFEL ET AL. 2006). Konflikte, die im Zusammenhang mit Waldbewirtschaftung entstehen, sind meist wegen ihrer hohen Komplexität nur schwer strukturierbar. Mit der Strukturierung des Problems (Unterteilung in einzelne Kriterienfelder) und der Hinterlegung der Argumente mit Simulationsergebnissen werden die Kernprobleme greifbar und lösbar. Die in dieser Arbeit vorgestellte multikriterielle Betrachtung von Waldbaustrategien ermöglicht den Dialog zwischen Organisationen mit verschiedenen Schwerpunkten.

Diese Arbeit kann einen Beitrag zur Schaffung von Transparenz leisten und als Entscheidungshilfe bei Konflikten herangezogen werden. Je nach Zielsetzung der einzelnen Konfliktparteien müßten einzelne Module jedoch verbessert werden (zum Beispiel die Beurteilung des Betriebsergebnisses). Auch die Ergänzung des Systems durch Hinterlegung von Risiko- oder Qualitätsmodellen kann sich in diesem Zusammenhang anschließen. Um Entscheidungsträger in allen Ebenen einer Organisation (operative, taktische, strategische Entscheidungsebene) bei der Entscheidungsfindung zu unterstützen, ist der Ausbau des Modells zu einem interaktiven Informationssystem notwendig. Mit dem Hinterlegen des hier vorgestellten Modells mit frei definierbaren Entscheidungsfunktionen käme man dieser Forderung näher. In Verbindung mit einer graphischen Benutzeroberfläche, die auch die Anpassung der Rahmenwerte und der Maßnahmenketten erlaubt, kann das hier vorgestellte Modell potentiellen Nutzern zugänglich gemacht werden. Das Durchführen von Sensitivitätsanalysen würde dann sogar die Optimierung von Waldbaustrategien für einzelne Parteien ermöglichen. 


\section{Inhaltsangabe}

In der vorliegenden Untersuchung wird eine Möglichkeit vorgestellt, forstliche Managementstrategien in einem Waldwachstumssimulator abzubilden. Die Umsetzung von vier Waldbaukonzepten wird dokumentiert, die Anwendung dieser Konzepte anhand von Beispielbeständen und einem großräumigen Untersuchungsgebiet dargestellt, und die Ergebnisse der Fortschreibungen werden anhand ausgewählter Auswertungsparameter diskutiert.

Im Hintergrund dieser Arbeit müssen die vielschichtigen Anforderungen, die an einen Wirtschaftswald gestellt werden, berücksichtigt werden. Wegen der Komplexität des Ökosystems Wald liegt das primäre Ziel dieser Arbeit darin, die Transparenz über den bei der Anwendung einer Managementstrategie - zu erwartenden Zustand eines Forstes zu erhöhen. Es können sowohl einzelne Bestände, als auch ganze Betriebe oder Landschaften analysiert werden.

Dabei müssen die Besonderheiten von Waldbaustrategien berücksichtigt werden. Zu diesen Besonderheiten gehören die Produktionsdauer, die Abhängigkeit von natürlichen Bedingungen, die Schwierigkeit der Ertragsbestimmung, die Produktion forstlicher Dienstleistung, die Bereitstellung einer forstlichen Infrastruktur, die Bewertung einzelner Bestände und des gesamten Forstbetriebs, der Kapitalumschlag und der hohe Eigenkapitalanteil, die große Flächenausdehnung sowie die Standortsgebundenheit des Betriebs (SPEIDEL 1984).

Waldbaustrategien entstehen vor dem Hintergrund des aktuellen Waldzustands, der örtlichen Gegebenheiten, langjähriger Erfahrungen, der erwarteten Kosten und Erlöse und der politischen Rahmenbedingungen. Sie bedienen sich auf der Ebene des operativen Managements waldbaulicher Maßnahmen, die sich auf die Endnutzung, die Jungwuchspflege, auf Läuterungen, Wertästung und die Durchforstung von Beständen beziehen.

Beim Vergleich zur Verfügung stehender planungsunterstützender Instrumente stellen sich Waldwachstumsmodelle auf Einzelbaumbasis als besonders geeignet heraus, um über eine angemessene Zeitspanne hinweg die Auswirkung forstlicher Eingriffe abzubilden. In den Einzelbaumwachstumsmodellen aus Europa findet man zahlreiche Abbildungen von gängigen Bewirtschaftungsformen.

In dieser Arbeit wurde mit dem Waldwachstumssimulator BWINPro gearbeitet. In den bestehenden Programmcode wurden Methoden eingebunden, die die Umsetzung von vier Waldbaustrategiebeispielen ermöglichen. Das Spektrum reicht von einer Ökonomie und Ökologie vereinenden Alternative (LÖWE), der Umsetzung naturnaher Bestockung (PNV) über eine ertragsorientierte Wahlmöglichkeit (ERTRAG) bis hin zu einer kaum steuernd eingreifenden Variante (PROZESS).

Die programmiertechnische Umsetzung verschiedener Waldbaukonzepte wird durch eine szenarienabhängige Auswahl an Maßnahmenelementen, die in einer Maßnahmenkette hintereinandergeschaltet werden, ermöglicht. Als Maßnahmenelemente werden Methoden bezeichnet, die zum Beispiel die Abbildung von Vornutzungs- oder Endnutzungseingriffen, 
Durchforstungen oder Pflanzungsmaßnahmen ermöglichen. Variantenspezifische Rahmenwerte steuern den Ablauf der Maßnahmenelemente. Sie beziehen sich zum Beispiel auf angestrebte Zielstärken, minimale und maximale Entnahmemengen oder auf angestrebte Mischungsverhältnisse einer Strategie.

Die Komponenten der Variante LÖWE sind so ausgewählt und konfiguriert, daß sie die waldbaulichen Strategien in den Niedersächsischen Landesforsten wiedergeben. Die 1991 erklärten Ziele des Konzepts LÖWE sind Gemeinnützigkeit, Nachhaltigkeit und Wirtschaftlichkeit. Nutz-, Schutz- und Erholungsfunktion des Waldes werden als gleichrangig betrachtet. Das Konzept beinhaltet die Förderung von Mischbeständen in Durchforstungen und auch durch Pflanzungen. Eine natürliche Verjüngung der Bestände wird jedoch bevorzugt. Um die Diversität zu erhöhen, werden Minderheiten geschützt und mögliche Habitatbäume aus der Nutzung genommen. Die vertikale Struktur profitiert von mäßigen hochdurchforstungsartigen Eingriffen und der Zielstärkennutzung in erntereifen Beständen. Die Variante sieht bis zu zwei Eingriffe im Jahrzehnt vor.

Mit der Variante PNV wird die Entwicklung der Baumartenzusammensetzung stark in Richtung der potentiell natürlichen Vegetation (pnV) (nach KAISER UND ZACHARIAS 2003) gesteuert. Es werden dazu solche Baumarten stark gefördert, die der pnV entsprechen. Wie LÖWE vollzieht auch das Szenario PNV alle fünf Jahre forstliche Eingriffe in den Beständen. Die Rahmenwerte orientieren sich an den Rahmenwerten von LÖWE. Lediglich der Freistellungsgrad der Bäume ist höher, um den Umbau in Richtung pnV zu beschleunigen.

Um eine ertragsorientierte Waldbehandlung umzusetzen, wird in der Variante ERTRAG ein Umbau der Istbestockungen in ertragsstarke Baumarten forciert. Desweiteren werden die Holzerntekosten durch stärkere Durchforstungen und längere Durchforstungsintervalle (alle zehn Jahre) sowie höhere Endnutzungsmassen gesenkt. Letztere können durch starke Durchforstungen, angepaßte Endnutzungsvolumina und baumartenabhängige Endnutzungszeiträume, an deren Ende die Nutzung der führenden Bestandesschichten steht, erreicht werden. Im Vergleich zu den beiden bereits vorgestellten Varianten verkürzen sich die Umtriebszeiten auf lange Sicht, weil die Bäume mit Erreichen eines geringeren BHDs endgenutzt werden. Die Wälder entwickeln sich bei dieser Variante zu gleichaltrigen Reinbeständen, in denen Minderheiten oder Habitatbäume nicht geschützt werden.

Bei der Variante PROZESS sollen natürliche Prozesse im Wald genutzt und unterstützt werden, indem zum Beispiel konkurrenzstarke Bäume zusätzlich gefördert werden. Es wird darauf verzichtet, bestimmte Mischungsverhältnisse bei den beteiligten Baumarten anzustreben, um die natürliche Entwicklungsdynamik nicht $z u$ stören. Die konkurrenzstärkeren Baumarten setzen sich im Bestand durch. Die Förderung von Bäumen durch die Entnahme ihrer Bedränger fällt im Vergleich zu den anderen Szenarien eher schwach aus. Es wird mit einem Eingriff im Jahrzehnt gearbeitet, um dem Bestand die Möglichkeit zu geben, sich zwischen diesen Behandlungen natürlich differenzieren zu können. Um in der Endnutzungsphase nur kleinflächige Störungen zu verursachen, werden zielstarke Bäume individuell genutzt. Dies reduziert die Verjüngungsmöglichkeiten für lichtbedürftige Baumarten. Pflanzungen gehören nicht zu diesem Konzept. Durch die 
Auswahl von Habitatbäumen wird der Totholzanteil der Bestände erhöht, und Lebensräume für Totholzbewohner werden geschaffen.

Das Simulationsinstrument verarbeitet gängige Forsteinrichtungsdaten aus einer Datenbank und ist in der Lage, verschiedene Auswertungsparameter zu liefern. Die Ausgabe erfolgt in eine Datenbank. Es wurden Auswertungsparameter ausgewählt, die es ermöglichen, die Entwicklung des Untersuchungsobjekts in bezug auf seine forstlichen Ressourcen, seine Produktionsfunktion, seine biologische Diversität und seine Zielerreichung zu beurteilen. Die Auswahl lehnt sich an den Katalog der gesamteuropäischen Kriterien einer nachhaltigen Forstwirtschaft an, der bei der dritten Ministerkonferenz zum Schutz der Wälder in Europa 1998 in Lissabon von den europäischen Forstministerien unter dem Namen Resolution L2 verabschiedet wurde.

Bevor die vier Szenarien auf einen Untersuchungsraum angewendet werden, wird zunächst die Entwicklung von drei für das Gebiet charakteristischen Beispielbeständen im Durchforstungsalter und einem Beispielbestand im Endnutzungsalter untersucht. Bei den Beispielbeständen im Durchforstungsalter handelt es sich um einen Bestand aus fünfzigjähriger Kiefer, einen Bestand aus dreißigjähriger Fichte und einen Kiefern-/ Fichtenmischbestand. Die Kiefer ist im letztgenannten Bestand fünfzig- und die Fichte dreißigjährig. Als Beispielbestand im Endnutzungsalter dient ein mit fünfzehnjähriger Buche unterbauter Bestand aus hundertzehnjähriger Kiefer.

In den Beispielbeständen gelingt es, anhand von Stammverteilungsplänen zu zeigen, daß die in den Simulationsläufen eingesetzten Durchforstungs- und Endnutzungsalgorithmen reale Eingriffen nachbilden können. In den Auswertungsparametern spiegelt sich wider, daß die Beispielbestände in den vier Szenarien deutlich verschiedene Entwicklungsrichtungen nehmen. Je nach Ausgangssituation kann die Entwicklung der Bestände mehr oder weniger beeinflußt werden. Zum Beispiel bietet der Bestand aus fünfzigjähriger Kiefer durch sein Alter und seine Bestandesstruktur erheblich bessere Umbaumöglichkeiten als die anderen Bestände. Durch Pflanzungsmaßnahmen ist es in diesem Bestand möglich, variantenspezifische Umbauszenarien zu simulieren. In den anderen Beständen ist keine Verjüngung möglich (wie in den anderen Beispielen für Durchforstungsbestände), oder die Verjüngung ist schon zu Simulationsbeginn festgelegt (wie im Bestand aus hundertzehnjähriger Kiefer).

Durch die Hintereinanderschaltung von Maßnahmenelementen zu den vier simulationsspezifischen Maßnahmenketten und durch den Rückgriff der Varianten auf spezifische Rahmenwerte gelingt es, Eingriffsart, Eingriffsstärke, Eingriffsintensität und die Zeitpunkte der Eingriffe nachvollziehbar zu steuern. Sowohl im Bereich forstlicher Ressourcen als auch im Hinblick auf die Biologische Diversität und den Zielerreichungsgrad kann die Bestandesentwicklung logisch schlüssig gelenkt werden. Im Hinblick auf die Bewertung der Produktionsfunktion stellt der vierzigjährige Simulationszeitraum eine Einschränkung dar. Variantenspezifische Einflüsse auf das Betriebsergebnis kommen innerhalb dieser Zeitspanne nur bedingt zum Tragen.

Nach der Analyse der Beispielbestände werden die Strategien auf ein Untersuchungsgebiet angewendet. Der 11610ha große Untersuchungsraum liegt in der Lüneburger Heide. Er zeichnet sich durch mäßig frische, relativ schwach 
nährstoffversorgte Standorte aus. Kieferndominierte Bestandestypen prägen aus waldhistorischen Gründen das Landschaftsbild; auf vielen Flächen wurde jedoch durch Pflanzungen ein Baumartenwechsel vorbereitet. Der Altersklassenaufbau der Bestände ist charakteristisch für ein Waldgebiet, das sich in der Phase des Vorratsaufbaus befindet.

Erwartungsgemäß steigt in allen vier Varianten der Derbholzvorrat innerhalb des Simulationszeitraums an. Die Auswirkung einer stark umsteuernden Durchforstungsstrategie auf den Vorrat wird bei der Betrachtung der Variante PNV deutlich: In dieser Variante wird der geringste Vorrat aufgebaut. Mit dem Vorrat steigt auch der erntekostenfreie Abtriebswert der Bestände an. Unterschiede zwischen den Varianten sind größtenteils auf die jeweiligen Durchforstungsstärken zurückzuführen. Da im Untersuchungsgebiet nur begrenzt Endnutzungen stattfinden, haben diese Eingriffe kaum Einfluß auf den durchschnittlichen Wert eines Bestandes des Untersuchungsgebiets. In Änderung der Durchmesserstruktur des Untersuchungsgebiets sind sowohl die Einflüsse der Durchforstungsstrategien als auch der Endnutzungsstrategien zu erkennen. Durch die hochdurchforstungsartigen Eingriffe bildet sich in allen Varianten eine zweigipflige Verteilung heraus. In der Variante ERTRAG werden die Bestände aufgrund ihrer Endnutzungsstrategie früher endgenutzt als in den anderen Varianten. Dies spiegelt sich einer geringeren Besetzung der Durchmesserklassen, die über dem Ziel-Dg dieser Variante liegen, wider.

Bei der monetären Bewertung der im Simulationszeitraum durchgeführten Eingriffe wird deutlich, daß die Pflanzungsmaßnahmen einen entscheidenden Einfluß auf betriebswirtschaftliche Kennzahlen haben. So profitiert zum Beispiel die Variante PROZESS im Vergleich $\mathrm{zu}$ den anderen Varianten von ihrem vollständigen Pflanzungsverzicht. Das Betriebsergebnis der Variante PNV wird durch großflächige Umbaumaßnahmen stark negativ beeinflußt. Auch die in der Variante ERTRAG getätigten Investitionen in die Pflanzung ertragsstarker Baumarten können sich innerhalb der simulierten vierzig Jahre noch nicht positiv auf die Auswertungsparameter auswirken.

Die biologische Diversität wird auf Landschaftsebene in allen Varianten erhöht. Zu beachten ist dabei, daß alle Varianten von den bis zum Aufnahmezeitpunkt der Forsteinrichtung schon vollzogenen Umbaumaßnahmen gleichermaßen profitieren. Diese erhöhen im Untersuchungszeitraum sowohl die vertikale Struktur als auch die Baumartenvielfalt.

Die Totholzbildungsrate ist stark vom jeweiligen Durchforstungskonzept abhängig. In den Vornutzungen in der Variante PNV wird zum Beispiel so stark in die Bestände eingegriffen, daß die natürliche Mortalität auf ein Minimum sinkt. In der eingriffsarmen Variante PROZESS wird dagegen die höchste Totholzmasse über die vierzig Jahre akkumuliert.

Durch die Auswahl von Habitatbäumen werden in den Varianten LÖWE und PROZESS Lebensräume für Totholzbewohner geschaffen. Die Nutzungsmassen werden durch den Entzug dieser Bäume aus der Nutzung nicht wesentlich beeinflußt.

Der Einfluß der vier Managementstrategien zeigt sich bei einer Betrachtung der Veränderung der Baumartenanteile deutlich. Die Variante LÖWE kommt den in dieser Variante angestrebten Mischungsverhältnissen am nächsten, da in großen Teilen des 
Untersuchungsgebiets schon vor Simulationsbeginn über Jahrzehnte nach diesem Konzept gewirtschaftet wurde. Auch die Änderungen in den Varianten ERTRAG und PNV sind beträchtlich. In der Variante ERTRAG fällt besonders die gelungene Erhöhung des Douglasien- und Fichtenanteils auf. Mit der Umsetzung der Strategie PNV erhöht sich der Buchenanteil erheblich. Die Variante PROZESS zeigt eine ähnliche Entwicklung wie die Variante LÖWE, da sie von den im voraus getätigten Pflanzungen profitiert. Ob diese Baumartenanteile in Zukunft zu halten sind, wird in Frage gestellt.

In einer vergleichenden Übersicht werden Auswertungsparameter aller untersuchten Bereiche („forstliche Ressourcen“, „Produktionsfunktion“, „biologische Diversität" und „Zielerreichung“) dargestellt und ein übergreifender Vergleich der Varianten ermöglicht. Die Gesamtbetrachtung muß vor dem Hintergrund gesehen werden, daß die Bestandesentwicklung nicht nur durch die variantenspezifischen Maßnahmen beeinflußt wird. Auch die waldbauliche Ausgangssituation hat Auswirkungen auf die Ergebnisse. Der Simulationszeitraum ist an übliche Planungszeiträume und nicht an die Produktionszeiträume angepaßt. Daraus resultiert, daß man die Varianten nicht abschließend bewerten kann, sondern nur Entwicklungstendenzen im Simulationszeitraum darstellt.

So schneidet zum Beispiel die Variante PROZESS am Ende des Simulationszeitraums in vielen Bereichen am besten ab. Die Entwicklung der Bestände profitiert in dieser Variante von den Pflanzungen und den Pflegemaßnahmen, die zu Beginn des Simulationszeitraums schon getätigt worden waren. Für die Zukunft ist jedoch zu erwarten, daß Bestände in dieser Variante zunehmend an Wert und Diversität verlieren. Die Variante PNV bleibt in fast allen Bereichen hinter den Ergebnissen der anderen Varianten zurück. Im Rahmen des Umbaus werden hohe Investitionen getätigt, die Vorräte niedrig gehalten und der Zuwachs der Bestände beeinträchtigt. Nicht nur in der Variante PNV, sondern auch in den Varianten LÖWE und ERTRAG ist das Betriebsergebnis zum Ende der vierzig Jahre stark negativ durch die Kosten des Bestandesumbaus beeinflußt. Hinzu kommt bei der Betrachtung der Variante ERTRAG, daß die Vorzüge dieser Variante (Anbau ertragsstarker Baumarten, kürzere Umtriebszeiten) im Untersuchungszeitraum nicht zum Tragen kommen. Außerdem werden betriebswirtschaftliche Vorteile durch hohe Nutzungsmassen in der Berechnung der Kennzahlen nicht ausreichend berücksichtigt. Die Variante LÖWE zeichnet sich durch ihre Ausgewogenheit aus, sie weist weniger Extremwerte als die anderen Varianten auf. Standortsgerecht werden dort ertragsstarke Baumarten gefördert, ohne auf Mischbestände, Minderheitenschutz und die Auswahl von Habitatbäumen zu verzichten.

Insgesamt kann der Ansatz als erfolgreich bezeichnet werden, waldbauliche Strategien in Maßnahmenelemente zu zerlegen, die in variantenspezifischen Maßnahmenketten zusammengesetzt und durch szenarienabhängige Rahmenwerte gesteuert werden. In allen Bereichen („forstliche Ressourcen“, „Produktionsfunktion“, „biologische Diversität“ und „Zielerreichung“) können szenarienabhängige Entwicklungen belegt werden, die mit der szenarienabhängigen Maßnahmenwahl und den individuell gesetzten Rahmenwerten begründet werden können. Es stellt sich jedoch die Frage, wie die Auswertung und Darstellung der Ergebnisse optimiert werden können, um dem Nutzer auch die langfristigen Vor- und Nachteile einer Strategie transparent zu machen. 
Vor dem Hintergrund der langfristigen forstlichen Produktionszeiträume wäre die Abbildung einer gesamten Umtriebszeit wünschenswert. Ob solche Informationen betriebliche Entscheidungen beeinflussen würden, ist jedoch ungewiß. Die Grenzen des Systems liegen neben der Darstellung eines auf vierzig Jahre begrenzten Simulationszeitraums auch darin, daß Kalamitäten in der Simulation nicht berücksichtigt werden. Auch langanhaltende extreme Wetterlagen (wie extreme Sommertrockenheit), Klimaveränderungen, der Einfluß von Stoffeinträgen aus der Atmosphäre oder die Folgen von Veränderungen des Grundwasserspiegels sind nicht Gegenstand der Simulation. Von einem störungsfreien Verlauf der Waldentwicklung kann man jedoch unter realen Bedingungen nicht ausgehen. Weiterhin muß angemerkt werden, daß es durch die Beschränkung der Simulationsflächen auf 0,25ha zu einer Verzerrung der Baumartengruppenanteile kommen kann. Eine Grenze des Systems liegt auch darin, daß jeder Bestand isoliert von den inn umgebenden Beständen betrachtet wird; bestandesübergreifende Maßnahmenplanung ist daher nicht möglich. Weiterhin stützt sich die betriebswirtschaftliche Auswertung auf Durchschnittskosten und Durchschnittserlöse der letzten Jahre; künftig geltende Kosten und Erlöse sind wegen fehlender Informationen über die kommende Marktentwicklung nicht vorhersagbar.

Weiterer Forschungsbedarf besteht bei Verbesserung des Systems in den Punkten Bestandesaufbau, Ablauf der Simulation sowie der Wahl und Berechnung der Ausgabeparameter.

Beim Bestandesaufbau sollten in Zukunft die von der Forsteinrichtung angegebenen Durchmesser korrigiert und Minderheitenanteile sicherer geschätzt werden. Auch die Festlegung der Modellflächengröße und die räumliche Verteilung der simulierten Baumobjekte kann noch optimiert werden.

Für den Ablauf der Simulation ist es gerade für die monetäre Bewertung der Varianten wünschenswert, den räumlichen Bezug der Bestände untereinander zu berücksichtigten. So können Kostendegressionseffekte berücksichtigt werden, die zum Beispiel durch Maschineneinsatz und geringeren organisatorischen Aufwand entstehen. Auch eine Ausdehnung des Simulationszeitraums wäre eine wertvolle Erweiterung des Programms. Ein weiterer Ansatzpunkt für die Verbesserung des Modells ist die flexiblere Gestaltung von Eingriffsintervallen. Denkbar ist auch eine Erweiterung der Auswahl an Maßnahmenelementen. Neben inhaltlichen Änderungen ist eine Verkürzung der Simulationslaufzeit anzustreben.

Um die Transparenz über die Auswirkung der Strategien auf die Bestände zu erhöhen, können einige Ausgabeparameter modifiziert werden. Zum Beispiel können die Erlösfunktionen überarbeitet werden. Für die betriebswirtschaftliche Bewertung im Rahmen der Produktionsfunktion würde die Einbindung einiger zusätzlicher Faktoren (zum Beispiel Planprozeßkosten) eine höhere Aussagekraft ermöglichen. Für die Bewertung des Kriteriums „biologische Diversität“ kann eine differenziertere Betrachtung der Landschaftselemente zu einer exakteren Bewertung der landschaftlichen Vielfalt führen. Außerdem kann die Vergleichbarkeit der anfallenden Totholzmengen mit Kalibrierungsfunktionen erhöht werden. 
In Verbindung mit einer graphischen Benutzeroberfläche, die auch die Anpassung der Rahmenwerte und der Maßnahmenketten erlaubt, kann das hier vorgestellte Modell potentiellen Nutzern zugänglich gemacht werden. Je nach Zielsetzung der einzelnen Konfliktparteien müßten einzelne Module jedoch verbessert werden. Das Durchführen von Sensitivitätsanalysen würde dann sogar die Optimierung von Waldbaustrategien für einzelne Parteien ermöglichen und das Programm zu einem Entscheidungsunterstützungssystem reifen lassen.

\section{Zusammenfassung}

\subsection{Vergleich forstlicher Managementstrategien - Umsetzung verschiedener Waldbaukonzepte in einem Waldwachstums- simulator}

In der vorliegenden Dissertation wird eine Möglichkeit vorgestellt, forstliche Managementstrategien in einem Waldwachstumssimulator abzubilden. Die Umsetzung von vier Waldbaukonzepten ist dokumentiert, die Anwendung dieser Konzepte wird dargestellt, und die Ergebnisse der Fortschreibungen werden anhand ausgewählter Nachhaltigkeitsparameter diskutiert. Das Ziel dieser Arbeit ist es, wichtige Aufschlüsse über die vom Waldbaukonzept abhängigen Entwicklungsmöglichkeiten von Wäldern zu geben.

Es wird mit dem Waldwachstumssimulator BWINPro gearbeitet, der die Bestände auf Einzelbaumbasis fortschreibt. Vier Waldbaukonzepte wurden exemplarisch in den bestehenden Programmcode integriert:

1. eine ertragsorientierte Option (ERTRAG),

2. ein Ökonomie und Ökologie vereinende Alternative (LÖWE),

3. die Umsetzung einer naturnaher Bestockung (PNV) und

4. eine kaum steuernd eingreifendes Konzept (PROZESS).

Die programmiertechnische Umsetzung verschiedener Waldbaukonzepte wird durch eine szenarienabhängige Auswahl an Maßnahmenelementen, die in einer Maßnahmenkette hintereinander geschaltet werden, ermöglicht. Als Maßnahmenelemente werden Methoden bezeichnet, die zum Beispiel die Abbildung von Vornutzungs- oder Endnutzungseingriffen, Durchforstungen oder Pflanzungsmaßnahmen ermöglichen. Variantenspezifische Rahmenwerte steuern den Ablauf der Maßnahmenkette. Sie beziehen sich zum Beispiel auf angestrebte Zielstärken, minimale und maximale Entnahmemengen oder auf angestrebte Mischungsverhältnisse einer Strategie.

Das Simulationsinstrument verarbeitet Daten aus einer relationalen Datenbank. Als Input dienen gängige Forsteinrichtungsdaten. Die Auswahl der Ausgabeparameter ist an den Katalog der gesamteuropäischen Kriterien einer nachhaltigen Forstwirtschaft angelehnt, der bei der dritten Ministerkonferenz zum Schutz der Wälder (MCPFE) in Europa 1998 in Lissabon verabschiedet wurde (Resolution L2). 
Die vier Szenarien werden exemplarisch sowohl auf einen 11610ha großen Untersuchungsraum, als auch repräsentative Beispielbestände aus der Lüneburger Heide angewendet. Die Simulationsläufe zeigen, daß die eingesetzten Algorithmen reale Eingriffe angemessen nachbilden. In den Auswertungsparametern spiegelt sich wider, daß Bestände in den vier Szenarien deutlich verschiedene Entwicklungsrichtungen nehmen. Je nach Ausgangssituation kann die Entwicklung der Bestände im Simulationszeitraum mehr oder weniger beeinflußt werden.

In Verbindung mit einer graphischen Benutzeroberfläche könnte das hier vorgestellte Modell potentiellen Nutzern in Zukunft zugänglich gemacht werden. Je nach Zielsetzung können einzelne Module dazu verbessert und auf die individuellen Bedürfnisse der Anwender zugeschnitten werden. In Zukunft kann das Programm durch eingebettete Sensitivitätsanalysen zu einem Entscheidungsunterstützungssystem reifen und die Optimierung von Waldbaustrategien für verschiedene Interessengruppen ermöglichen.

\subsection{Comparison of Forest Management Strategies - Realization of Silviculture Concepts within a Forest Growth Simulator}

This dissertation deals with a possibility to reproduce management strategies in a forest growth simulator. The implementation of four silviculture concepts is documented, the application of these concepts is described, and the results of the simulations are discussed on the basis of selected sustainability parameters. The aim of this thesis is to offer valuable clues to the different possibilities of forest development according to the application of various silviculture concepts.

The forest growth simulator used was BWINPro which predicts stand development on single tree basis. Four silviculture concepts have exemplarily been integrated into the existing program:

1. a yield-oriented option (ERTRAG),

2. an alternative which unites economy and ecology (LÖWE),

3. the realisation of a nature-oriented growing stock (PNV)

4. a concept that reduces steering interferences to a minimum (PROZESS).

Within the program, I have realized various silviculture concepts by using a scenariodependent selection of treatments which have been stringed together in a treatment chain. These treatments are translated into methods which represent, for example, intermediate and final cuttings, thinning or planting in a stand. The chain of treatments is steered by specific framework values depending on the concept which is used. These values, for example, define the threshold for selective-thinning, minimum and maximum timber extraction volumes, or the mixing proportion of species at which the strategy aims.

The simulation instrument processes data from a relational data base. As an input, ordinary management planning data is used. The output parameters are derived from the Pan-European criteria for sustainable forest management as given by the third Ministerial 
Conference on the Protection of Forests in Europe (MCPFE) 1998 in Lisbon (Resolution L2).

The four scenarios are applied both to a 11610ha investigation area and to representative stand-examples from an area in northern Germany called Lüneburger Heide. The simulations show that real treatments are emulated adequately by the algorithms I have developed. The analysis parameters reveal that stands clearly show different trends in development within each of the four scenarios. Depending upon the stand condition at the starting point of the simulation, its development can be affected more or less by the treatments within the simulated time period.

In combination with a graphical user surface, the presented model could be made implementable for potential users. Modules could be improved and adjusted to their needs. Through an embedded sensitivity analysis the program could mature in the future into a decision support system which could realize the optimization of forest management strategies for different lobbies. 


\section{Literaturverzeichnis}

Abetz, P. (1975): „Entscheidungshilfe für die Durchforstung von Fichtenbeständen“, Allgemeine Forstzeitschrift (30), S. 666-667

Abetz, P. (1980): „Zum Konzept einer Z-Baum-orientierten Kontrollmethode“, Allgemeine Forst- und Jagdzeitung (151/4-5), S. 65-68

Abetz, P. (1982): „Zuwachsreaktion von Z-Bäumen und Durchforstungsansätze bei Auslesedurchforstung in Fichtenbeständen“, AFZ (47), S. 1444-1450

Abetz, P. u. Klädtke, J. (2002): „The target tree management system“, Forstwissenschaftliches Centralblatt (121), S. 73-82

Albert, M. (2000): „Ein fuktionaler Höhenergänzungsalgorithmus für Einzelbaumwachstumsmodelle“, Tagungsbericht der Sektion Ertragskunde DVFFA (Kaiserslautern), S. 32-43

Albert, M. (2002): „Generating management alternatives for multi-species stands using the decision support system BWINPro", Managing Forest Ecosystems: Continious Cover Forestry Assessment, Analysis, Scenarios, Kluwer Academic Publishers/ Dordrecht, Boston, London, S. 187-202

Albert, M. (2003): „Analyse und multikriterielle Bewertung alternativer Waldentwicklungsszenarien mit Hilfe des Analytischen Hierarchieprozesses“, Tagungsbericht der Sektion Ertragskunde DVFFA (Torgau), S. 256-270

Albert, M. u. Guericke, M. (2003): „Zur Prognose waldbaulicher Behandlungsvarianten in Mischbeständen mit einzelbaumbasierten Durchforstungsmodellen“, Forstarchiv (74), S. 79-89

Albrecht, L. (1990): „Naturreservate in Bayern“, Schriftenreihe Bayerisches Staatsministerium für Ernährung, Landwirtschaft und Forsten (1), $219 \mathrm{~S}$.

Alder, D.; Hammond, D.S. u. Hughell, D.A. (2002): „A growth model and harvest allocation algorithm for timber and non-timber product management planning in Iwokrama Forest, Guyana", Managing Forest Ecosystems: Continious Cover Forestry - Assessment, Analysis, Scenarios, Kluwer Academic Publishers/ Dordrecht, Boston, London, S. 175-185

Ammer, U. u. Utschick, H. (2004): „Folgerungen aus waldökologischen Untersuchungen auf hochproduktiven, nadelholzreichen Standorten für eine an Naturschutzzielen orientierte Waldwirtschaft", Forst und Holz (59/3), S. 119-128

Anonymus (1996): „Umweltgruppen stellen Zertifizierung für die deutsche Forstwirtschaft vor“, HolzZentralblatt (122/97-98), 1469

Arbeitskreis Standortskartierung in der Arbeitsgemeinschaft Forsteinrichtung (1985): „Forstliche Wuchsgebiete und Wuchsbezirke in der Bundesrepublik Deutschland“, Landwirtschaftsverlag (Münster Hiltrup), $170 \mathrm{~S}$.

Arney, J.D. (1972): „Computer simulation of Douglas-fir tree and stand growth“, PhD Thesis, Oregon State University/ Corvallis, 79 S.

Assmann, E. (1961): „Waldertragskunde“, BLV Verlagsgesellschaft/ München, Bonn, Wien, 490 S.

Assmann, E. (1963): „Geben unsere Buchen-Ertragstafeln den Gang des Volumenzuwachses richtig wieder?", Forstwissenschaftliches Centralblatt (3/4), S. 64-75

Assmann, E. u. Franz, F. (1963): „Vorläufige Fichten-Ertragstafel für Bayern“, Institut für Ertragskunde der Forstlichen Forschungsanstalt/ München, $104 \mathrm{~S}$. 
Assmann, E. u. Franz, F. (1965): „Vorläufige Fichten-Ertragstafel für Bayern“, Forstwissenschaftliches Centralblatt (84/1), S. 13-42

Augusto, L.; Ranger, J.; Binkley, D. und Rothe, A. (2002): „Impact of several common tree species of European temperate forests on soil fertility“, Annals of Forest Science (59), S. 233-253

Baker, W.L. u. Cai, Y. (1992): „The r.le programs for multiscale analysis of landscape structure using the GRASS geographical information system", (7/4), S. 291-302

Balcar, P. (2004): „Erkenntnisse aus der Naturwaldforschung für den naturnahen Waldbau“, AFZ/ Der Wald (18), S. 983-985

Bartelink, H.H. (2000): „A growth model for mixed forest stands“, Forest Ecology and Management (134), S. $29-43$

Barth, E. (2002): „Untersuchung zur Ökonomie von drei Waldbewirtschaftungs-Konzepten - am Beispiel ausgewählter Nadelholzbestände im Stadtforstamt Uelzen“, Diplomarbeit, Universität Lüneburg, 94 S.

Bartha, D. (2004): „Die Naturnähe der Wälder - Bewertung auf Bestandesebene“, Allgemeine Forst- und Jagdzeitung (175/1-2), S. 9-17

Baumgart, A. (2004): „Zur Bestimmung der Naturnähe von Wäldern des nordostdeutschen Tieflandes“, Forst und Holz (59/9), S. 438-441

Bella, I.E. (1971): „A new competition model for individual trees“, Forest Science (17/3), S. 364-372

Bergel, D. (1986): „Douglasien-Ertragstafel für Nordwestdeutschland 1985“, Allgemeine Forst- und Jagdzeitung (157/3-4), S. 49-59

Biber, P. (1996): „Konstruktion eines einzelbaumorientierten Wachstumssimulators für FichtenBuchenmischbestände im Solling“, Berichte des Forschungszentrums Waldökosysteme (A/142), 252 S.

Biber, P. u. Herling, H. (2002): „Modellierung der Verjüngungsdynamik als Bestandteil von einzelbaumorientierten Wachstumsmodellen“, Tagungsbericht der Sektion Ertragskunde DVFFA (Schwarzburg), S. 194-216

Bieling, C. u. Schraml, U. (2004): „Was ist der Natur näher als der Wald? Zur Wahrnehmung des Waldzustands durch private Eigentümer", Allgemeine Forst- und Jagdzeitung (175/3), S. 41-49

Biging, G. S. u. Dobbertin, M. (1995): „Evaluation of competition indices in individual tree growth models“, Forest Science (41/2), S. 360-377

Böckmann, T. (2004a): „Möglichkeiten und Grenzen von Waldwachstumsmodellen aus Sicht der Forstlichen Praxis", Berichte der Freiburger Forstlichen Forschung, Waldwachstumsmodelle für Prognosen in der Forsteinrichtung (50), Eigenverlag der FVA/ Freiburg, S. 95-102

Böckmann, T. (2004b): „Forsteinrichtung - als Institution unverzichtbar“, Allgemeine Forst- und Jagdzeitung (175/7-8), S. 157-163

Boddenberg, J. u. Henkel, A. (2004): „Aufbau eines Netzes von nutzungsfreien Wäldern Naturwaldparzellen in Thüringen“, AFZ/ Der Wald (1), S. 7-10

Bonnemann, A. (1939): „Der gleichaltrige Mischbestand von Kiefer und Buche“, Mitteilungen aus Forstwirtschaft und Forstwissenschaft (5), S. 439-483

Bonnemann, A. (1967): „Waldbauliche Terminologie“, Schriftenreihe der Forstlichen Fakultät der Universität Göttingen und der Niedersächsischen Forstlichen Versuchsanstalt, J.D. Sauerländer's Verlag/ Frankfurt am Main (40), $44 \mathrm{~S}$. 
Bormann, F.H. u. Likens, G.E. (1979a): „Pattern and process in a forested ecosystem“, Springer/ New York, $253 \mathrm{~S}$.

Bormann, F.H. u. Likens, G.E. (1979b): „Catastrophic disturbance and the stady state in northern hardwood forests“, American Science (67), S. 660-669

Bossel, H. (1994): „TREEDYN 3 forest simulation model - mathematical model, program documentation and simulation results", Berichte des Forschungszentrums Waldökosysteme, Universität Göttingen (B/35), $118 \mathrm{~S}$.

Bossel, H. (1995): „TREEDYN 3 forest simulation model“, Ecological Economics (90/3), S. 187-227

Botkin, D.B.; Janack, J.F. u. Wallis, J.R. (1972): „Some ecological consequences of a computer model of forst growth“, Journal of Ecology (60/3), S. 849-872

Box, E.O. u. Meentemeyer, V. (1991): „Geographic modeling and modern ecology“, Modern Ecology Basic and applied aspects, S. 773-804

Bradley, R.T.; Christie J.M. u. Johnson D.R. (1966): „Forest management tables“, Forestry Commission BookletForestry Commission/ London (16), $212 \mathrm{~S}$.

Bray, J.R. (1956): „Gap-phase replacement in a maple-basswood forest“, Ecology (37/3), S. 598-600

Brecht, B. (1965): „Herr Puntila und sein Knecht Matti: Volksstück“, Suhrkamp/ Frankfurt, 132 S.

Brouwer, R. u. Ek, R. (2004): „Integrated ecological, economic and social impact assessment of alternative flood control policies in the Netherlands", Ecological Economics (50), S. 1-21

Buckmann, R.E. (1962): „Growth and yield of red pine in Minesota“Technical Bulletin, St. Paul, MN: U.S. Department of Agriculture, Forest Service, Lake States Forest Experiment Station (1272), $50 \mathrm{~S}$.

Bundesanstalt für Geowissenschaften und Rohstoffe (1976): „Geologische Übersichtskarte [1: 200000; Blatt CC; 3118 Hamburg-West)“Hannover

Bundesministerium für Ernährung, Landwirtschaft und Forsten (2000): „Nationales Forstprogramm für Deutschland“" (Broschüre), $58 \mathrm{~S}$.

Bundesministerium für Ernährung, Landwirtschaft und Verbraucherschutz (2003): „Das nationale Waldprogramm für Deutschland“, http://nwp-online.de/, 15.06.2010

Burkhart, H.E.; Farrar, K.D.; Amateis, R.L. u. Daniels, R.F. (1987): „Simulation of individual tree growth and stand development in loblolly pine plantations on cutover, site-prepared areas", PublicationSchool of Forestry and Wildlife Resources, Virginia Polytechnic Institute and State University (FWS-1-87), 47 S.

Burschel, P. (1974): „Die Durchforstung aus heutiger Sicht“, Forstarchiv (45/2-3), S. 21-28

Burschel, P. u. Huss, J. (1997): „Grundriß des Waldbaus“, Pareys Studientexte/ 2. Auflage, Parey/ Berlin (49), $487 \mathrm{~S}$.

Busse, J. (1935): „Gruppendurchforstung“, Silva (19), S. 145-147

BWaldG (1975): „Bundeswaldgesetz“, BGBI I (Vom 2. Mai 1975 (BGBI. I S. 1037), zuletzt geändert durch Artikel 2 Abs. 1 des Gesetzes vom 26. August 1998), S. 1037+2521

Cancino, J. u. von Gadow, K. (2002): „Stem number guide curves for uneven-aged forests development and limitations", Managing Forest Ecosystems: Continious Cover Forestry - Assessment, Analysis, Scenarios, Kluwer Academic Publishers/ Dordrecht, Boston, London, S. 163-175

Christensen, M.; Hahn, K.; Mountford, E.P.; Odor, P.; Standovar, T.; Rozenbergar, D.; Diaci, J.; Wijdeven, S.; Meyer, P.; Winter, S. u. Vrska, T. (2005): „Dead wood in European beech (Fagus syvatica) forest reserves", Forest Ecology and Management (210/1-3), S. 267-282 
Clark, P.J. u. Evans, F.C. (1954): „Distance to nearest neighbour as mesure of spatial relationship in populations“, Ecology (35/4), S. 445-453

Clutter, J.L. (1963): „Compatible Growth and Yield Models for Loblolly Pine“, Forest Science (9/3), S. 354371

Clutter, J.L. u. Bennett, F.A. (1965): „Diameter distributions in old-field slash pine plantations“, Georgia Forestry Research Council Reputation (13), $9 \mathrm{~S}$.

Cotta, H. (1815): „Abriß einer Anweisung zur Vermessung, Beschreibung, Schätzung und forstwirtschaftlichen Einteilung der Waldungen“, Arnold/ Dresden, $31 \mathrm{~S}$.

Curtis, J.T. (1959): „The vegetation of Wisconsin“, University Wisconsin Press/ Madison, 657 S.

Dale, M.R.T. (2000): „Lacunarity analysis of spatial pattern: A comparison“, Landscape Ecology (15), S. 467-478

Daniels, R.F. u. Burkhart, H.E. (1975): „Simulation of individual tree growth and stand development in managed loblolly pine plantations", PublicationSchool of Forestry and Wildlife Resources, Virginia Polytechnic Institute and State University (FWS-5-75, 69 S.

Daume, S. (1998): „A knowledge-based system to model thinnings in central European forests“, M.Sc. thesis, Department of Artificial Intelligence/ University of Edinburgh, S. 237-249

Daume, S. u. Robertson, D. (2000): „A heuristic approach to modeling thinnings“, Silva Fennica (34/3), S. 237-249

Degenhardt, A. (1998): „Simulation von Baumverteilungsmustern in Mischbeständen“, Beiträge für Forstwirtschaft und Landschaftsökologie, Landesforschungsanstalt Eberswalde (32/3), S. 133-146

Dengler, A. (1992a): „Der Wald und seine Vegetationsform und seine Bedeutung für den Menschen“, Waldbau/ 6. Auflage (bearbeitet von Röhrig, E. und Bartsch, N.), Parey/ Berlin (1), $350 \mathrm{~S}$.

Dengler, A. (1992b): „Baumartenwahl, Bestandesbegründung und Bestandespflege“, Waldbau/ 6. Auflage (bearbeitet von Röhrig, E. und Bartsch, N.), Parey/ Berlin (2), 314 S.

Döbbeler, H. u. Spellmann, H. (2002): „Methodological approach to simulate and evaluate silvicultural treatments under climate change“, Forstwissenschaftliches Centralblatt (121), 52-69

Döbbeler, H.; Nagel, J.; Albert, M. u. Schmidt, M. (2003): „BWINPro Programm zur Bestandesanalyse und Prognose", Handbuch zur Version 6.2, Niedersächsische Forstliche Versuchsanstalt Göttingen, $124 \mathrm{~S}$.

Duda, H.A.A. (2004): „Prognose der Vorratsentwicklung - Simulation des LÖWE Konzepts im Vergleich zu den Ertragstafeln“, Tagungsbericht der Sektion Forstliche Biometrie und Informatik DVFFA (Freising), S. 171-177

Duda, H.A.A. (2005): „Die Umsetzung verschiedener Waldbehandlungskonzepte in einem Waldwachstumssimulator", Tagungsbericht der Sektion Ertragskunde DVFFA (Freising), S. 72-76

Duffner, W. (2004): „Ökonomie im Dienste der Ökologie“, Forst und Holz (59/2), S. 51-54

Dursky, J. (2000): „Einsatz von Waldwachstumssimulatoren für Bestand, Betrieb und Großregion“, Habilitationsschrift, Forstwissenschaftliche Fakultät der Technischen Universität München, 223 S.

Eberhard, J. (1914): „Die Grundlagen naturgemäßer Bestandesbegründung“, Forstwissenschaftliches Centralblatt, S. 75-86

Eckmüllner, O. u. Fleck, W. (1989): „Begleitdokumentation zum Wachstumsprogramm WASIM Version 1.0“, Institut für Forstliche Ertragslehre, Universität für Bodenkultur/ Wien, $30 \mathrm{~S}$. 
Ek, A.R u. Monserud, R.A. (1974): „Trials with program FOREST: growth and reproduction simulation for mixed species even or uneven-aged forest stands", Research notes, Growth models for tree and stand simulation: International Union of Forestry Research Organizations Working Party (30), Department of Forest Yield Research, S. 55-73

Ek, A.R. u. Dudek, A. (1980): „Development of individual tree based stand growth simulators: progress and applications", Staff paper series, St. Paul, Minnesota: Department of Forest Resources, College of Forestry and the Agricultural Experiment Station, Institute of Agriculture, Forestry and Home Economics, University of Minnesota (20), $25 \mathrm{~S}$.

Emborg, J.; Christensen, M. u. Heilmann-Clausen, J. (2000): „The structural dynamics of Suserup Skov, a near- natural temperate deciduous forest in Denmark", Forest Ecology and Management (126), S. 173-189

Encke, B.G. (2004): „ANW und Betriebswirtschaft - Der naturgemäße Wald als Chance für alle Besitzarten“, AFZ/ Der Wald (18), S. 960-962

EStG (2005): „Einkommensteuergesetz“, BGBI III FNA 611-1 (vom 16.10.34 (RGBI_I_34,1005), in der Fassung der Bekanntmachung vom 19.10.2005

Fabrika, M. u. Dursky, J. (2005): „Algorithms and software solution of thinning models for growth simulator SIBYLA“, Tagungsbericht der Sektion Ertragskunde DVFFA (Freising), S. 77-92

Fabrika, M. u. Dursky, J. (2006): „Implementing Tree Growth Models in Slovakia“, Sustainable Forest Management, Springer/ Berlin, Heidelberg, S. 315-342

Fähser, L. (1995): „Das Konzept der naturnahen Waldnutzung im Stadtforstamt Lübeck“, Der Dauerwald (12), S. 2-6

FBVA - Forstliche Bundesversuchsanstalt (1994): „Instruktionen für die Feldarbeit der Österr. Forstinventur", Forstliche Bundesversuchsanstalt/ Wien, $194 \mathrm{~S}$.

Frank, A. (1969): „Was soll die Ausscheidung von Intensitätsstufen?“, AFZ (24/27), S. 558-559

Franz, F. (1967): „Das EDV-Programm STOET - zur Herleitung mehrgliedriger Standort-Leistungstafeln“ (Manuskriptdruck/ München), unveröffentlicht

Franz, F. (1969): „Die Beurteilung des Produktionsvermögens als Entscheidungshilfe bei Intensitätsstufenkalkulation“, AFZ (24/27), S. 550-554

Franz, F. (1974): „Zur Fortentwicklung der Durchforstungsverfahren aus der Sicht der Waldertragskunde“, Forstarchiv (45/2-3), S. 28-34

Freistaat Thüringen/Landesforstverwaltung (2006a): „Naturnahe Waldwirtschaft“, http://www.thueringen.de/imperia/md/content/folder/waldoekolog/naturnahewaldwirtschaft.pdf, 15.06.2006

Freistaat Thüringen/Landesforstverwaltung (2006b): „Waldbaugrundsätze in Thüringen“, http://www.thueringen.de/imperia/md/content/folder/waldschutz/waldbaugrundsaetze.pdf, 15.06.2006

Gatzojannis, S.; Stefanidis, P. u. Kalabokidis, K. (2001): „An inventory and evaluation methodology for non-timber functions of forests", Mitteilungen der Abteilung für Forstliche Biometrie, Abteilung für forstliche Biometrie/ Universität Freiburg (2001/1), 49 S.

Golser, M. u. Hasenauer H. (1997): „Predicting juvenile height growth in uneven-aged mixed species stands in Austria“, Forest Ecology and Management (97), S. 133-146 
GRASS Development Team (2005): „GRASS 6.0 Users Manual, ITC-irst, Trento, Italy“, http://grass.itc.it/grass60/manuals/html_grass60, 15.06.2016

Gruss, K. (2004): „PEFC- Kontrollstichprobe dargestellt am Beispiel der Zertifizierungsregion Niedersachsen“, Forst und Holz (59/7), S. 357-360

Hallenbarter, D. u. Hasenauer, H. (2003): „Durchforstungen in Fichtenbeständen: Eine Analyse mit Hilfe des Waldwachstumsmodells MOSES“, Centralblatt für das gesamte Forstwesen (120/3-4), S. 211229

Hanewinkel M. (1996): „Anforderungen an ein Konzept: Überführung von Fichtenreinbeständen in Bestände mit Dauerwaldstruktur", AFZ/ Der Wald (26), S. 1440-1446

Hanewinkel, M. (1998): „Plenterwald und Plenterüberführung: Modellstudien zu planungstechnischen, waldbaulichen und ökonomischen Aspekten am Fallbeispiel des Wuchsgebietes Schwarzwald“, Schriften aus dem Institut für Forstökonomie, Fakultät für Forst- und Umweltwissenschaften/ Universität Freiburg (9), 256 S.

Hanewinkel, M. (2001): „Neuausrichtung der Forsteinrichtung als strategisches Managementinstrument“, Allgemeine Forst- und Jagdzeitung (172/11), S. 202-211

Hanewinkel, M. (2003): „Entscheidungsunterstützung bei komplexen betrieblichen Managementaufgaben am Beispiel von Waldumbau/ Überführung und Risikomanagement", Schriften aus dem Institut für Forstökonomie, Fakultät für Forst- und Umweltwissenschaften/ Universität Freiburg (22), $328 \mathrm{~S}$.

Haniel, J. u. Kleinschmit, H. (1992): „Möglichkeiten und Grenzen: Zielstärkennutzung in den niedersächsischen Landesforsten“, AFZ (11), S. 588-593

Harting, G.L. (1795): „Anweisung zur Taxation der Forste, oder zur Bestimmung des Holzertrags der Wälder", Georg-Ludwig-Hartig-Stiftung/ Wiesbaden, 200 S.

Hasenauer, H. (1994): „Ein Einzelbaumwachstums-Simulator für ungleichaltrige Fichten-Kiefern- und Buchen-Kiefern- und Buchen-Fichtenmischbestände“, Forstliche Schriftenreihe, Österreichische Gesellschaft für Waldökosystemforschung und Experimentelle Baumforschung/ Wien (8), $152 \mathrm{~S}$.

Hasenauer, H. (1997): „Dimensional relationships of open-grown trees in Austria“, Forest Ecology and Management (96), S. 197-206

Hasenauer, H. (2004): „ITM - Implementing Tree Growth Models as Forest Management Tools“, Final Report (CD)

Hasenauer, H. (2006a): „Sustainable Forest Management“, Springer/ Berlin, Heidelberg, 398 S.

Hasenauer, H. (2006b): „Concepts Within Tree Growth Modeling“, Sustainable Forest Management, Springer/ Berlin, Heidelberg, S. 3-18

Hasenauer, H. u. Kindermann, G. (2002): „Methods for assessing regeneration establishment and height growth in uneven-aged mixed species stands", Forestry - An International Journal of Forest Research (75/4), S. 385-394

Hasenauer, H. u. Kindermann, G. (2006): „Modeling Regeneration in Even and Uneven-Aged Mixed Species Forests", Sustainable Forest Management, Springer/ Berlin, Heidelberg, S. 167-194

Hauhs, M.; Kastner-Maresch, A. u. Rost-Siebert, K. (1995): „A model relating forest growth to ecosystemscale budgets of energy and nutrients", Ecological Modeling (83/1-2), S. 229-243

Hemm, M.; Bruchner, A.K. u. Warkotsch, W. (2003): „Software als Planungsinstrument im forsttechnischen Bereich“, AFZ/ Der Wald (26), S. 1332-1334 
Hempel, G. u. Wätzig, H. (1994): „Arbeitsbericht über die Strukturierung einer Wissensbasis mit Hilfe eines Entwicklerwerkzeugs - dargestellt an der Konzeption für ein Expertensystem „Holzmeßkunde““', Tagungsbericht der Sektion Forstliche Biometrie und Informatik DVFFA (Ljubljana), S. 137-163

Hessisches Ministerium für Landesentwicklung, Wohnen, Landwirtschaft, Forsten und Naturschutz/ Wiesbaden (1993): „RIBES 93“, Hessisches Ministerium für Landesentwicklung, Wohnen, Landwirtschaft, Forsten und Naturschutz/ Wiesbaden, $18 \mathrm{~S}$.

Heyer, C (1841): „Die Waldertrags-Regelung“, B. C. Ferber/ Gießen, 264 S.

Hillmann, M. (2002): „Forsteinrichtung, Waldinventur, Betriebssteuerung“, Forst und Holz (57/10), S. 303306

Hillmann, M. (2006): „15 Jahre flächendeckende Waldinventur im Privatwald“, AFZ - Der Wald (1), S. 8 u. 9

Hinrichs, L. (2004): „Untersuchungen zur Generierung von Behandlungsvarianten für Buchen-Fichten Mischbestände“, Tagungsbericht der Sektion Ertragskunde DVFFA (Stift Schlägl), S. 43-50

Hinrichs, L. (2005): „Konstruktion eines Durchforstungsmodells zur Generierung multipler Waldentwicklungspfade für Buchen-Fichten-Mischbestände", Tagungsbericht der Sektion Ertragskunde DVFFA (Freising), S. 14-22

Hinze, F.-J.; Höfle, H. C.; Jordan, H.; Mengeling, H.; Meyer, K.-D.; Rohde, P. u. Streif, H. (1995): "Quartärgeologische Übersichtskarte von Niedersachsen und Bremen 1:500 000“, Niedersächsisches Landesamt für Bodenforschung/ Hannover

Huber, M. (1993): „Die Gruppendurchforstung - Vorstellung eines Durchforstungsverfahrens mit Vergleichen zur Auslesedurchforstung“, Der Dauerwald (9), S. 30-47

Hui, G.Y. u. Albert, M. (2004): „Stichprobensimulation zur Schätzung nachbarschaftsbezogener Strukturparameter in Waldbeständen“, Allgemeine Forst- und Jagdzeitung (175/10-11), S. 199-209

Hundeshagen, J.C. (1826): „Die Forstabschätzung auf neuen wissenschaftlichen Grundlagen“, H. Laupp/ Tübingen, $428 \mathrm{~S}$.

Huss, J. (1992): „Was ist Waldbau auf ökologischer Grundlage?“, AFZ (2), S. 456-464

Hyink, D.M. u. Moser, J.W. (1983): „A generalized framework of projecting forest yield and structure using diameter distributions", Forest Science (29/1), S. 85-95

Jacobsen, J.B.; Möhring, B. u. Wippermann, C. (2004): „Business economics of conversion and transformation - a case study of Norway spruce in Northern Germany“, European Forest Institute Research Report (18), S. 225-252

Janßen, G. (2000): „Von der Verwüstung zum naturnahen Wirtschaftswald - ein Gebot rationeller, ökonomisch sinnvoller Forstwirtschaft", Forst und Holz (55/18), S. 579-587

Janßen, G. (1992): „Forstpolitische Aspekte einer ökologischen Ausrichtung der Forstwirtschaft in Niedersachsen", AFZ (47/11), S. 562-565

Johann, K. (1982): „Der „A-Wert“ - ein objektiver Parameter zur Bestimmung der Freistellungsstärke von Zentralbäumen“, Tagungsbericht der Sektion Ertragskunde, DVFFA (Weibersbrunn), S. 146-158

Johann, K. (1983): „Beispiele „A-Wert“ gesteuerter Z-Baum Freistellung. Anwendung im Versuchswesen und in der Praxis", Tagungsbericht der Sektion Ertragskunde, DVFFA (Neuhaus/ Solling), S. 3.13.14 
Kahn, M. (1995a): „Rule based modeling of thinning regimes for a distance dependent single tree growth simulator by implementing a fuzzy logic controller", Congress Report, IUFRO World Congress/ Tampere (Finland) (S 4.01), S. 29-84

Kahn, M. (1995a): „Die Fuzzy Logik basierte Modellierung von Durchforstungseingriffen“, Allgemeine Forst- und Jagdzeitung (166), S. 169-176

Kahn, M. u. Pretzsch, H. (1997): „Das Wuchsmodell SILVA - Parametrisierung der Version 2.1 für Reinund Mischbestände aus Fichte und Buche“, Allgemeine Forst- und Jagdzeitung (168/6-7), S. 115123

Kaiser, T. (1996): „Die potentielle natürliche Vegetation als Planungsgrundlage im Naturschutz“, Natur und Landschaft (71/10), S. 435-439

Kaiser, T. (1999): „Anwendung des Konzepts der potentiellen natürlichen Vegetation in der praktischen Landschaftsplanung", NNA Berichte (12/2), S.105-112

Kaiser, T. u. Zacharias, D. (2003): „PNV-Karten für Niedersachsen auf Basis der BÜK 50“, Informationsdienst Naturschutz Niedersachsen, Hildesheim (1/2003), $68 \mathrm{~S}$.

Karjalainen, T.; Spiecker, H. u. Laroussinie, O. (1999): „Causes and consequences of accelerating tree growth in Europe“, European Forest Institute proceedings, European Forest Institute/ Joensuu (27), $285 \mathrm{~S}$.

Kato, F. (1973): „Begründung der qualitativen Gruppendurchforstung“, Habilitationsschrift, Institut für forstliche Betriebswirtschaftslehre der Universität Göttingen, 132 S.

Kato, F. u. Mülder, D. (1979): „Qualitative Gruppendurchforstung der Buche - Grundsätze, Wertentwicklung nach 10 Jahren, praktische Anleitung“, Allgemeine Forst- und Jagdzeitung (150/6), S. 105-111

Kato, F. u. Mülder, D. (1983): „Qualitative Gruppendurchforstung der Buche“, Allgemeine Forst- und Jagdzeitung (154/8), S. 139-145

Kato, F. u. Mülder, D. (1988): „Qualitative Gruppendurchforstung der Buche - Wertentwicklung nach 20 Jahren“, Allgemeine Forst- und Jagdzeitung (159/1-2), S. 4-9

Kenk, G. u. Hradetzky, J. (1985): „Behandlung und Wachstum der Douglasie in Baden-Württemberg“, Mitteilungen der Forstlichen Versuchs- und Forschungsanstalt Baden-Württemberg, Ministerium für Ländlichen Raum, Ernährung, Landwirtschaft und Forsten (113), $89 \mathrm{~S}$.

Kenk. G. (1984): „Werteichenproduktion und ihre Verbesserung in Baden-Württemberg“, AFZ (39/17-18), S. $428-429$

Kindermann, G.; Hasenauer, H. u. Gasch, J. (2002): „Ankommen und Wachstum von Naturverjüngung in Mischbeständen“, Centralblatt für das gesamte Forstwesen (119/2), S. 159-186

Kirsch, W. (1997): „Wegweiser zur Konstruktion einer evolutionären Theorie der strategischen Führung: Kapitel eines Theorieprojektes“, Münchener Schriften zur angewandten Führungslehre/ 2. Auflage, Verlag Barbara Kirsch (80), 469 S.

Kittenberger, A. (2003): „Generieren von Baumverteilungsmustern“, Diplomarbeit, Universität für Bodenkultur/ Wien, $79 \mathrm{~S}$.

Klädtke, J. (1993): „Konstruktion einer Z-Baum-Ertragstafel am Beispiel der Fichte“, Mitteilungen der Forstlichen Versuchs- und Forschungsanstalt Baden-Württemberg, Ministerium für Ländlichen Raum, Ernährung, Landwirtschaft und Forsten (32), $18 \mathrm{~S}$. 
Klädtke, J. u. Yue, C. (2003): „Produktionszielorientierte Entscheidungshilfe für die Bewirtschftung ungleichaltriger Fichten-Tannen-Wälder und Plenterwälder", Allgemeine Forst- und Jagdzeitung (174/10-11), S. 196-206

Klein, H. (2000): „Probleme beim Anbau standortsfremder Gehölze im Wald“, http://www.waldklein.de/wbau/exot_l.pdf, 15.06.2006

Kleinschmit, H. (1999): „Naturschutzkonzept in den niedersächsischen Landesforsten“, Forst und Holz (54/20), S. 627-630

Knauft, F.J. (2000): „Entwicklung von Methoden zur GIS-gestützten Visualisierung von Waldentwicklungsszenarien“, Dissertation, Fakultät für Forstwissenschaften und Waldökologie der Georg-August-Universität/ Göttingen, 127 S.

Koepke, D. (1999): „Zielbildung im Privatwald als individueller Problemlösungsprozess“, Dissertation, Fakultät für Forstwissenschaften und Waldökologie der Georg-August-Universität/ Göttingen, 175 S.

Konitzer, A. (2000): „Waldumbaumaßnahmen und ihre Auswirkungen - eine GIS-gestützte Untersuchung am Beispiel des Niedersächsischen Harzes“, Dissertation, Fakultät für Forstwissenschaften und Waldökologie der Georg-August-Universität/ Göttingen, S. 18-33

Koop, H. (1982): „Waldverjüngung, Sukzessionsmosaik und kleinstandörtliche Differenzierung infolge spontaner Waldentwicklung", Berichte der Internationalen Symposien der Internationalen Vereinigung für Vegetationskunde (25), Cramer/ Vaduz, S. 235-267

Köpf, E. U. (2002): „Forstpolitik: 61 Übersichten und Tabellen“, Ulmer/ Stuttgart, 320 S.

Korol, M. u. von Gadow, K. (2004): „Zur quantitativen Beschreibung von Fichtenkronen im Gebiet der ukrainischen Karpaten“, Centralblatt für das gesamte Forstwesen (121/2), S. 81-90

Korol, R.L.; Milner, K.S. u. Running, S.W. (1996): „Testing a mechanistic model for predicting stand and tree growth“, Forest Science (42/2), S. 139-153

Korpel, S. (1992): „Urwälder in den Westkarpaten“, Fischer/ Stuttgart, 310 S.

Köstler, J.N. (1969): „Waldbauliche Realisationsmöglichkeiten“, AFZ (24/47), S. 555-558

Kowarik, I. (1987): „Kritische Anmerkungen zum theoretischen Konzept der potentiellen natürlichen Vegetation mit Anregungen zu einer zeitgemäßen Modifikation“, Tuexenia (7), S. 53-57

Kramer, H. (1976): „Begriffe der Forsteinrichtung“, Schriftenreihe der Forstlichen Fakultät der Universität Göttingen und der Niedersächsischen Forstlichen Versuchsanstalt, J.D. Sauerländer's Verlag/ Frankfurt am Main (48), $80 \mathrm{~S}$.

Kramer, H. u. Akca, A. (1982): „Leitfaden zur Waldmeßlehre“, J.D. Sauerländer's Verlag/ Frankfurt am Main, $266 \mathrm{~S}$.

Kremser, W. (1990): „Niedersächsische Forstgeschichte“, Rothenburger Schriften (Sonderband 32), 965 $\mathrm{S}$.

Kremser, W. (1972): „Die Aufforstung der niedersächsischen Heidegebiete aus kulturhistorischer und kulturgeographischer Sicht"Rothenburger Schriften (36)

Kronauer, H. (1992): „UNCED-Gipfel: Aufbruch oder nur Tanz um das Goldene Kalb“, AFZ (22), S. 11991200

Kroth, W. (1974): „Wirtschaftliche Aspekte der Durchforstung“, Forstarchiv (45/2-3), S. 39-42

Krug, A. u. Klein, H. (1997): „Zu: Waldposition des Bundes polemisch und nicht haltbar“, AFZ/ Der Wald (2), S. 87 
Krutzsch, H. (1950): „Der naturgemäße Wirtschaftswald“, AFZ (5/8), S. 85-87

Krutzsch, H. u. Weck, J. (1934): „Bärenthoren 1934 - Der naturgemäße Wirtschaftswald“, Verlag von J. Neumann/ Neudamm, $162 \mathrm{~S}$.

Landesforstverwaltung Nordrhein-Westfalen (1990): „Waldwirtschaft 2000: Gesamtkonzept für eine ökologische Waldbewirtschaftung des Staatswaldes in Nordrhein-Westfalen", Landesforstverwaltung Nordrhein-Westfalen, $16 \mathrm{~S}$.

Landsberg, J.J. u. Waring, R.H. (1997): „A generalised model of forest productivity using simplified concepts of radiation-use efficiency, carbon balance and partitioning", Forest Ecology and Management (95/3), S. 209-228

Ledermann, T. (2002a): „Ein Einwuchsmodell aus den Daten der Österreichischen Waldinventur 19811996“, Centralblatt für das gesamte Forstwesen (119/1), S. 40-76

Ledermann, T. (2002b): „Using logistic regression to model tree selection preferences for harvesting in forests in conversion", Managing Forest Ecosystems: Continuous Cover Forestry - Assessment, Analysis, Scenarios (4), Kluwer Academic Publishers/ Dordrecht, Boston, London, S. 203-216

Ledermann, T. u. Stage, A.R. (2001): „Effects of competitor spacing in individual-tree indices of competition", Canadian journal of forest research (31), S. 2143-2150

Ledermann, T. u. Sterba, H. (2006): „Evaluating Management Regimes and Their Impact on Commercial Timber Supply Using an Individual-Tree Growth Model and Scenario Analysis“, Sustainable Forest Management, Springer/ Berlin, Heidelberg, S. 195-210

Lee, Y. (1967): „Stand models for lodgepole pine and limits to their application“, PhD Thesis, Faculty of Forestry, University of British Columbia/ Vancouver, $333 \mathrm{~S}$.

Lemm, R. (1991): „Ein dynamisches Forstbetriebs- Simulationsmodell“, Dissertation, Professur für Forsteinrichtung und Waldwachstum der ETH/ Zürich, $235 \mathrm{~S}$.

Lemm, R. (2004): „Ein Modell für alle Fälle“, Freiburger Forstlichen Forschung (Berichte), Waldwachstumsmodelle für Prognosen in der Forsteinrichtung (50), Eigenverlag der FVA/ Freiburg, S. 32-48

Leuschner, C. (1997): „Das Konzept der potentiellen natürlichen Vegetation (PNV): Schwachstellen und Entwicklungsperspektiven“, Flora (192), S. 379-391

Lewandowski, A.u. von Gadow, K. (1997): „Ein heuristischer Ansatz zur Reproduktion von Waldbeständen“, Allgemeine Forst- und Jagdzeitung (168/9), S. 170-174

Lin, J.Y. (1970): „Gesamtkonzept für eine ökologische Waldbewirtschaftung des Staatswaldes“, PhD Thesis, Duke University/ Durham (North Carolina), $182 \mathrm{~S}$.

Lockow, K.W. (1995): „Neue Ertragstafel für die Roterle“, Der Wald (45), S.268-271

Lockow, K.W. (1998): „Aufbau, Anwendung und waldbauliche Behandlung: Die neue SandbirkenErtragstafel“, AFZ/ Der Wald (5), S. 258-261

Loiskekoski, M. u. Halko, L. (2000): „European List of Criteria and Most Suitable Quantitative Indicators“, Ministerial Conference on the Protection of Forests in

Europehttp://www.mmm.fi/english/forestry/policy/minkonf/criteria.htm, 15.06.2006

Merker, K. (1997): „Ein Controllingsystem 'Naturgemäße Waldwirtschaft'“, Schriften der Forstökonomie, Sauerländer's Verlag/ Frankfurt a.M. (17), 212 S.

Merker, K. (1998): „Chancen und Risiken einer Strategie 'Umstellung auf Naturgemäße Waldwirtschaft"', Forst und Holz (53/16), S. 495-500 
Merker, K. u. Spellmann, H. (2000): „Nachweis forstlicher Nachhaltigkeit - Das EU-Life-Projekt der LFV Niedersachsen", Forst und Holz (55/2), S. 51-55

Meyer, P. (1997): „Probleme und Perspektiven der Naturwaldforschung am Beispiel Niedersachsens“, Forstarchiv (68/3), S. 87-98

Meyer, P. (2000): „Strukturelle Diversität und waldbauliche Eingriffe - Entwicklung der DurchmesserDiversität von Buchen- und Fichtenbeständen in Abhängigkeit von der Eingriffsstärke“, Tagung der Sektion Waldbau (Sachsen-Anhalt), S. 31-48

Meyer, P. u. Spellmann, H. (1997): „Prozeßschutz-Konzept aus der Sicht der Naturwaldforschung“, AFZI Der Wald (25), S. 1344-1346

Meyer, P. u. Unkrig, W. (2001): „Bestandes- und Verjüngungsdynamik im Naturwald 'Meninger Holz' in den Jahren 1988 bis 1999“, NNA-Berichte (2), S. 167-174

Meyer, P.; Unkrig, W. u. Griese, F. (2000): „Dynamik der Buche (Fagus sylvatica L.) in nordwestdeutschen Naturwäldern", Forst und Holz (55/15), S. 470-477

Ministerial Conference (1993): „Resolution H1: general guidelines for the sustainable management of forests in Europe", Second Ministerial Conference on the Protection of Forests in Europe, 16-17 June 1993/ Helsinki (Finland), http://www.mcpfe.org/resolutions/helsinki/resolution_h1.pdf, 15.06.2006, $5 \mathrm{~S}$.

Ministerial Conference (2003): „Conference Proceedings“, Fourth Ministerial Conference on the Protection of Forests in Europe, 28-30 April 2003/ Vienna (Austria), 247 S.

Ministerium für Ernährung, Landwirtschaft, Forsten und Fischerei (1996): „Ziele und Grundsätze einer naturnahen Forstwirtschaft in Mecklenburg-Vorpommern“, Sonderausgabe, Ministerium für Ernährung, Landwirtschaft, Forsten und Fischerei/ Mecklenburg- Vorpommern, $11 \mathrm{~S}$.

Mintzberg, H.; Ahlstrand, B. u. Lampel, J. (2002): „Strategy Safari: eine Reise durch die Wildnis des strategischen Managements", Redline Wirtschaft bei Ueberreuter/ Wien, 456 S.

Mitchell, K.J. (1969): „Simulation of the growth of uneven-aged stands of white spruce“, Yale University School of Forestry, Bulletin Yale University/ New Haven (75), $48 \mathrm{~S}$.

Mitchell, K.J. (1975): „Dynamics and simulated yield of Douglas-fir“, Forest Science Monograph (17), 39 $\mathrm{S}$.

Mitscherlich, G. (1952): „Der Tannen-Fichten-(Buchen)-Plenterwald“, 42 S.

Möhring, B. (2004a): „Nachhaltige Forstwirtschaft - nachhaltig erfolgreich'“, Allgemeine Forst- und Jagdzeitung (175/9), S. 165-170

Möhring, B. (2004b): „Betriebswirtschaftliche Analyse des Waldumbaus“, AFZ/ Der Wald (18), S. 963

Möhring, B.; Rüping, U., Leefken, G. u. Ziegler, M. (2006): „Die Annuität - ein 'missing link' der Forstökonomie", Allgemeine Forst- und Jagdzeitung (177/2), S. 21-29

Monserud, R.A. (1975): „Methodology for simulating Wisconsin northern hardwood stand dynamics“, PhD Thesis, University of Wisconsin/ Madison, $156 \mathrm{~S}$.

Monserud, R.A. u. Ek, A.R. (1977): „Predicting of understory tree height growth in northern hardwood stands", Forest Science (23/3), S. 391-400

Monserud, R.A. u. Sterba, H. (1996): „A basal area increment model for individual trees growing in evenand uneven-aged forest stands in Austria", Forest Ecology and Management (80), S. 57-80

Monserud, R.A. u. Sterba, H. (1999): „Modeling individual tree mortality for Austrian forest species“, Forest Ecology and Management (113), S. 109-123 
Moog, M.; Brabänder, H.D. (1994): „Vertragsnaturschutz in der Forstwirtschaft“, Schriften zur Forstökonomie, Universität Göttingen (3), 199 S.

Mosandl, R. u. Felbermeier, B. (2003): „Der neue Dreiklang im Waldbau: Ökologie, Sozioökonomie und Technologie“, Forst und Holz (58/6), S. 139-143

Moser, W. (1972): „Dynamics of an Uneven-Aged Forest Stand“, Forest Science (18/3), S. 184-191

Moser, W. u. Hall, F. (1969): „Deriving growth and yield functions for uneven-aged forest stands“, Forest Science (15/2), S. 183-188

Mrosek, M.; Kies, U. u. Schulte, A. (2005): „Clusterstudie Forst und Holz Deutschland 2005“, HolzZentralblatt (113/84), S. 1113

Müldner, D. (1995): „Naturnahe Waldnutzung in Mitteleuropa - Zum Aufsatz von Knut Sturm im Dauerwald-Heft 12“, Der Dauerwald (13), S. 4-23

Müldner, D. u. Greger, O. (1995): „Überlegungen zur Forstentwicklung der naturgemäßen Waldwirtschaft unter besonderer Berücksichtigung der Gruppendurchforstung“, Der Dauerwald (12), S. 33-42

Müller, W. (1994): „Der ökologische Umbau der Wälder in der Lüneburger Heide“, Forst und Holz (49/14), S. $396-398$

Nagel, J. (1995): „BWERT: Programm zur Bestandesbewertung und zur Prognose der Bestandesentwicklung", Tagungsbericht der Sektion Ertragskunde DVFFA (Joachimsthal), S. 184198

Nagel, J. (1996): „Anwendungsprogramm zur Bestandesbewertung und zur Prognose der Bestandesentwicklung“, Forst und Holz (51/3), S. 76-78

Nagel, J. (1997): „BWINPro Program for standard analysis and prognosis“, Users manual for version 3.0, Niedersächsische Forstliche Versuchsanstalt/ Göttingen, $121 \mathrm{~S}$.

Nagel, J. (1999): „Konzeptionelle Überlegungen zum schrittweisen Aufbau eines waldwachstumskundlichen Simulationssystems für Nordwestdeutschland“, Schriften aus der Forstlichen Fakultät der Universität Göttingen und der Niedersächsischen Forstlichen Versuchsanstalt, J.D. Sauerländer's Verlag/ Frankfurt am Main (128), $122 \mathrm{~S}$.

Nagel, J. (2002a): „Das Open Source Entwicklungsmodell - eine Chance für Waldwachstumssimulatoren“, Tagungsbericht der Sektion Ertragskunde DVFFA (Schwarzburg), S. 1-6

Nagel, J. (2002b): „TreeGrOSS: Tree Growth Open Source Software“, http://treegross.sourceforge.net/, 15.06.2006

Nagel, J. (2004): „Nutzungsplanung in Rein- und Mischbeständen“, Allgemeine Forst- und Jagdzeitung (175/7-8), S. 150-156

Nagel, J. (2005): „Integriertes Handbuch des Simulators: Modellfunktionen und Koeffizienten des Forest Simulators BWINPro Version 7.0“, http://treegross.sourceforge.net/, 15.06.2006

Nagel, J. u. Schmidt, M. (2006): „The Silvicultural Decission Support System BWINPro“, Sustainable Forest Management, Springer/ Berlin, Heidelberg, S. 59-63

Nagel, J.; Albert, M. u. Schmidt, M. (2002): „Das waldbauliche Prognose- und Entscheidungsmodell BWINPro 6.1 - Neuparametrisierung und Modellerweiterungen“, Forst und Holz (57/15-16), S. 486493

Natur- und Umweltschutz-Akademie des Landes Nordrhein- Westfalen (1999): „BuchennaturwaldReservate - unsere Urwälder von morgen"NUA- Seminarbericht (4), 52 S. 
Naturland, Greenpeace, BUND und Robin Wood (1996): „Der Wald hat ein Problem. Wir haben eine Lösung." (Broschüre)

Neter, J.; Maynes, S.E. (1970): „On the appropriateness of correlation coefficient with a 0.1 dependent variable", Journal of the American Statistical Association (65/330), S. 501-509

Newnham, R.M. (1964): „The development of a stand model for Douglas-fir“, PhD Thesis, University of British Columbia/ Vancouver, $201 \mathrm{~S}$.

Niedersächsische Forstliche Versuchsanstalt (2002a): „Bericht zu den Grundsätzen der Bestandesbegründung unter Berücksichtigung von Standort, Baumart und waldbaulicher Ausgangslage Teil I-II“, Bericht, Abteilung Waldwachstum, S. 66-78

Niedersächsische Forstliche Versuchsanstalt u. Niedersächsisches Forstplanungsamt (2002b): „Indikatoren nachhaltiger Forstwirtschaft - Bericht über das deutsche EU-Life Teilprojekt“, Schriftenreihe Waldentwicklung in Niedersachsen, Wolfenbüttel (10), $171 \mathrm{~S}$.

Niedersächsische Forstliche Versuchsanstalt (2003): „Naturwälder in den Landesforsten - Grundlagen, Betreuung, Forschung“ (Materialien zum Naturschutz-Lehrgang), $17 \mathrm{~S}$.

Niedersächsische Landesforsten (1994): „Langfristige Ökologische Waldbauplanung für die niedersächsischen Landesforsten“, Runderlaß des ML vom 05.05.1994, 38 S.

Niedersächsische Landesforstverwaltung (1989): „Entscheidungshilfen für die Pflege von Eichenbeständen“Merkblattsammlung (22), $18 \mathrm{~S}$.

Niedersächsische Landesforstverwaltung (1995): „Wertastung“ Merkblattsammlung (31), 18 S.

Niedersächsische Landesforstverwaltung (1987): „Anweisung zur Betriebsregelung (Forsteinrichtung) in den Niedersächsischen Landesforsten B.A.87“, Niedersächsische Landesforsten, 20 S.

Niedersächsische Landesforstverwaltung (1991): „Langfristige Ökologische Waldentwicklung in den Landesforsten“, Programm der Landesregierung, Niedersächsische Landesregierung Hannover, $49 \mathrm{~S}$.

Niedersächsische Landesforstverwaltung (2004): „Langfristige Ökologische Waldentwicklung Richtlinie zur Baumartenwahl“, Aus dem Walde, Hannover (54), $145 \mathrm{~S}$.

Niedersächsische Landesforstverwaltung (2006): „Merkblattsammlung“, Bestellung oder Download unter http://www.landesforsten.de/, 15.06.2006

Niedersächsisches Forstplanungsamt (2005): „Der Wald in Niedersachsen - Ergebnisse der Bundeswaldinventur II", Aus dem Walde/ Hannover (55), 44 S.

Niedersächsisches Forstplanungsamt (1998): „Ganzflächige Biotopkartierung: allgemeine Einführung und Kartierhinweise" (Broschüre), $29 \mathrm{~S}$.

Niedersächsisches Forstplanungsamt (1987): „Hilfstafeln für die Forsteinrichtung: zusammensgestellt für den Gebrauch in den Niedersächsischen Landesforsten“, Loseblattsammlung/ Wolfenbüttel, 158 $\mathrm{S}$.

Niedersächsisches Ministerium für Ernährung, Landwirtschaft und Forsten (2004): „Pflanzenzahlen bei der künstlichen Bestandesbegründung in den Landesforsten“, Nds. MBI Nr. 17/1998, S. 676-677

Nieschulze, J.; Böckmann, T.; Nagel, J. u. Saborowski, J. (2005): „Herleitung von einzelbestandesweisen Informationen aus Betriebsinventuren für die Zwecke der Forsteinrichtung“, Allgemeine Forst- und Jagdzeitung (176/9-10), S. 169-176

NWaldG (2002): „Niedersächsisches Gesetz über den Wald und die Landschaftsordnung“, Nds. GVBI (Vom 21 März 2002), S. 112 
Oesten, G u. Roeder, A. (2002): „Management von Forstbetrieben (1)“, www.forstbuch.de, 363 S.

Ostwald, E. (1931): „Grundlinien einer Waldrententheorie, d.h. einer im Anhalt an das relative WaldrentenMaximum entwickelten forstlichen Reinertragstheorie“, W.F. Häcker/ Riga, 351 S.

Otto, H. J. (1989): „Langfristige Ökologische Waldbauplanung für die niedersächsischen Landesforsten“, Aus dem Walde, Hannover (42/1), $442 \mathrm{~S}$.

Otto, H. J. (1990): „Die forstlichen Entwicklungen und Ziele einer naturnahen Waldpflege“, AFZ (45/2829), S. 723-725

Otto, H. J. (1991): „Langfristige Ökologische Waldbauplanung für die niedersächsischen Landesforsten“, Aus dem Walde, Hannover (43/2), $527 \mathrm{~S}$.

Otto, H. J. (1992): „Ökologische Grundlagen des Regierungsprogramms“, AFZ (47/11), S. 566-568

Otto, H. J. (1995a): „Zielorientierter Waldbau und Schutz sukzessionaler Prozesse“, Forst und Holz (50/7), S. $203-209$

Otto, H. J. (1995b): „Die Verwirklichung des LÖWE-Regierungsprogramms“, AFZ (19), S. 1028-1031

P\&P Baumschulen GmbH \& Co KG (2006): „Preisliste für Forstpflanzgut“, Bestellung unter http://www.baumschule.de/preisliste/angebot.html, 15.06.2006

Pacala S. W.; Canham, D.D.; Saponara, J.; Silander, J.A. Jr.; Kobe, R.K.; Ribbens, E. (1996): „Forest models defined by field measurements: estimation, error analysis and dynamics“, Ecological Monographs (66/1), S. 1-43

Paterak B. (1999): „Anforderungen an PNV-Karten aus Sicht der Landschaftsplanung“, NNA Berichte (12/2), S. 102-104

Paterak, B.; Bierhals, E. u. Preiss, A. (2001): „Hinweise zur Ausarbeitung und Fortschreibung des Landschaftsrahmenplans"Informationen des Naturschutz Niedersachsens (21/3), S. 121-192

Pausch, R. (2005): „Ein System-Ansatz zur Untersuchung von Zusammenhängen zwischen Waldstruktur, Arbeitsvolumina und Kosten der technischen und biologischen Produktion in Forstrevieren ost- und nordbayerischer Mittelgebirge", Forstliche Forschungsberichte München, Institut für Forstliche Arbeitswissenschaft und Angewandte Informatik/ Universität München (199), 301 S.

Pechmann, H. (1974): „Der Einfluß der Durchforstung auf die Holzqualität“, Forstarchiv, (45/2-3), S. 34-38

Peiechocki, R.; Wiersbinski, N.; Potthast, T. u. Ott, K. (2004): „Vilmer Thesen zum 'Prozessschutz'“, Natur und Landschaft (79/2), S. 53-56

Petersen, R. u. Guericke, M. (2004): „Untersuchungen zur Wuchsdynamik und Verjüngung der Fichte in Plenterwaldstrukturen“, Forst und Holz (59/2), S. 58-62

Pfau, W. (2001): „Strategisches Management“, München, 171 S.

Philipp, K. (1926): „Die Umstellung der Wirtschaft in den badischen Staats-, Gemeinde- und Körperschaftswaldungen“, Lang/ Karlsruhe, 79 S.

Pienaar, L.V. u. Turnbull, K.J. (1973): „The Chapman-Richards generalization of Bertalanffy's growth model for basal area growth and yield in even-aged stands", Forest Science (19/1), S. 2-22

Pistorius, T. (2004): „Die fehlende Integration des Forstsektors in die europäische Klimaschutzstrategie und Handlungsoption für die Forstwissenschaft", Forst und Holz (59/4), S. 183-189

Pommerening, A. (2002): „Approaches to quantifying forest structures“, Forestry - An International Journal of Forest Research (75/3), S. 305-324 
Prentice, I.C.; Cramer, W.; Harrison, S.P.; Leemanns, R.; Monserud, R.A. u. Solomon, A.M. (1992): „A global biome model based on plant physiology and dominance, soil properties and climate", Journal of Biogeography (19), S. 117-143

Pretzsch, H. (1992): „Konzeption und Konstruktion von Wuchsmodellen für Rein- und Mischbestände"Forstliche Forschungsberichte München (115), $332 \mathrm{~S}$.

Pretzsch, H. (1993): „Analyse und Reproduktion räumlicher Bestandesstrukturen - Versuche mit dem Strukturgenerator STRUGEN“, Schriften aus der Forstlichen Fakultät der Universität Göttingen und der Niedersächsischen Forstlichen Versuchsanstalt, J.D. Sauerländer's Verlag/ Frankfurt am Main (114), $87 \mathrm{~S}$.

Pretzsch, H. (1994): „Zum Einfluß des Baumverteilungsmusters auf den Bestandeszuwachs“, Tagungsbericht der Sektion Forstliche Biometrie und Informatik DVFFA (Ljubljana), S. 164-196

Pretzsch, H. (1996): „Strukturvielfalt als Ergebnis waldbaulichen Handelns“, Allgemeine Forst- und Jagdzeitung (167/11), S. 213-221

Pretzsch, H. (1997): „Analysis and modeling of spatial stand structures. Methodological considerations based on mixed beech-larch stands in Lower Saxony", Forest Ecology and Management (97), S. 237- 253

Pretzsch, H. (2000): „Die Regeln von Reineke, Yoda und das Gesetz der räumlichen Allometrie“, Allgemeine Forst- und Jagdzeitung (171/11), S. 205-210

Pretzsch, H. (2001): „Modellierung des Waldwachstums“, Parey/ Berlin, $341 \mathrm{~S}$.

Pretzsch, H. (2002): „Grundlagen der Waldwachstumsforschung“, Parey/ Berlin, 414 S.

Pretzsch, H. (2003): „Strategische Planung der Nachhaltigkeit auf Forstbetriebsebene - Beitrag der Waldwachstumsforschung", Forstwissenschaftliches Centralblatt (122), S. 231-249

Pretzsch, H. u. Kahn, M. (1995): „Modeling Forests in a Complex Environment - Modeling growth of Bavarian mixed stands in a changing environment", Congress Report, IUFRO World Congress/ Tampere (Finland) (II), S. 234-248

Pretzsch, H. u. Sodtke, R. (2004): „Theory and concept of decision support systems and decision-making in forest management supported by tree growth modeling", Tree Growth models for forest management in Europe, Springer/ Berlin, Heidelberg, S. 211-233

Pretzsch, H.; Biber, P. u. Dursky, J. (2002): „The single tree-based stand simulator SILVA: construction, application and evaluation", Forest Ecology and Management (162), S. 3-21

Pretzsch, H.; Biber, P.; Dursky, J.; von Gadow, K.; Hasenauer, H.; Kändler, G.; Kenk, G.; Kublin, E.; Nagel, J.; Pukkala, T.; Skovsgaard, J.P.; Sodtke, R. u. Sterba, H. (2002): „Recommendations for standardized documentation and further development of forest growth simulators", Forstwissenschaftliches Centralblatt (121), S. 138-151

Pretzsch, H.; Biber, P.; Dursky, J.; von Gadow, K.; Hasenauer, H.; Kändler, G.; Kenk, G.; Kublin, E.; Nagel, J.; Pukkala, T.; Skovsgaard, J.P.; Sodtke, R. u. Sterba, H. (2006): „Standardizing and Categorizing Tree Growth Models“, Sustainable Forest Management, Springer/ Berlin, Heidelberg, S. $39-58$

Pretzsch, H.; Utsching, H. u. Sodtke, R.M. (2006): „Applications of Tree Growth Modeling in Decision Support for Sustainable Forest Management“, Sustainable Forest Management, Springer/ Berlin, Heidelberg, S. 131-150 
Projektgruppe Naturwaldreservate des Arbeitskreises Standortskartierung in der Arbeitsgemeinschaft Forsteinrichtung (1993): „Empfehlung für die Einrichtung und Betreuung von Naturwaldreservaten in Deutschland“, Forstarchiv (64/3), S. 122-129

Pukkala, T. (1987): „Simulation model for natural regeneration of Pinus sylvestris, Picea abies, Betula pendula and Betula pubenscens", Silva Fennica (21), S. 37-53

Pukkala, T. (1988): „Effect of spatial distribution of trees on the volume increment of a young Scots pine stand“, Silva Fennica (22/1), S. 1-17

Pukkala, T. (1989): „Methods to describe the competition process in a tree stand“, Scandinavian Journal of Forest Research (4), S. 187-202

Pukkala, T. (2002): „Multi-objective forest planning“, Managing Forest Ecosystems: Continuous Cover Forestry - Assessment, Analysis, Scenarios (6), Kluwer Academic Publishers/ Dordrecht, Boston, London, 207 S.

Pukkala, T. (2006): „The Use of Multi-Criteria Decision Analysis and Objective Optimisation in Forest Planning“, Sustainable Forest Management, Springer/ Berlin, Heidelberg, S. 263-284

Pukkala, T. u. Kolström, T. (1992): „A stochastic spatial regeneration model for Pinus silvestris“, Scandinavian Journal of Forest Research (7), S. 377-385

Pukkala, T. u. Miina, J. (1997): „A method for stochastic multiobjective optimization of stand management", Forest Ecology and Management (98), S. 189-203

Rammetsteiner, E. (2001): „Demonstration von Methoden zur Erfassung der Nachhaltigkeit“, Forst und Holz (56/15), S. 459-463

Reineke, L.H. (1933): „Prefecting a stand density index for even aged forests“, Journal of Agricultural Research (46/7), S. 627-638

Reininger, H. (1987): „Zielstärken-Nutzung oder die Plenterung des Altersklassenwaldes“, Österreichischer Agrarverlag/ Wien, $163 \mathrm{~S}$.

Remmert, H. (1987): „Sukzessionen im Klimax-System“, Verhandlungen der Gesellschaft für Ökologie/ Gießen (XVI), S. 27-33

Remmert, H. (1988): „Naturschutz“, Springer/ Berlin, Heidelberg, 202 S.

Remmert, H. (1990): „Das Mosaik-Zyklus-Konzept der Ökosysteme“, NNA Berichte Schneverdingen (3/3), S. $110-117$

Remmert, H. (1991): „The mosaik-cycle concept of ecosystems“, Ecological studies, Springer/ Berlin, Heidelberg, New York, $168 \mathrm{~S}$.

Ribbens, E.; Silander, J.A. Jr. u. Pacala, S.W. (1994): „Seedling recruitment in forests: calibrating models to predict patterns of tree seedling dispersion“, Ecology (75/6), S. 1794-1806

Richter, J. (2004): „Gar keine Kahlschläge mehr?“, Forst und Holz (59/2), S. 54-57

Riederich, E.F. (1999): „Anbautechnische Untersuchungen in forstlichen Schnellwuchsplantagen und Demonstration des Leistungsvermögens schnellwachsender Baumarten“, Modellvorhaben „Schnellwachsende Baumarten“, Landwirtschaftsverlag/ Münster, S. 19-150

Riehl, W. H. (1857): „Land und Leute (1)“, Cotta/ Stuttgart, S. 48

Ripken, H. (1992): „Rationalisierungsmöglichkeiten in der biologischen Produktion des Forstbetriebs“, AFZ (47/11), S. 569-573

Ripken, H. (2004): „Kritische Betrachtung zur Multifunktionalität in der Waldbewirtschaftung“, Forst und Holz (59/3), S. 99-104 
Schädelin, W. (1934): „Die Durchforstung als Auslese- und Veredelungsbetrieb höchster Wertleistung“, Haupt/ Bern, $124 \mathrm{~S}$.

Scherzinger, W. (1990): „Naturschutz im Wald. Qualitätsziel einer dynamischen Waldentwicklung“, Ulmer/ Stuttgart, $447 \mathrm{~S}$.

Schmidt, M. (2001): „Prognosemodelle für ausgewählte Holzqualitätsmerkmale wichtiger Baumarten“, Dissertation, Fakultät für Forstwissenschaften und Waldökologie der Georg-August-Universität/ Göttingen, 296 S.

Schmidt, M. u. Nagel, J. (2006): „Evaluating Individual Tree Growth Models“, Sustainable Forest Management, Springer/ Berlin, Heidelberg, S. 151-164

Schmidt, M.; Böckmann, T. u. Nagel, J. (2006): „The Use of Tree Models for Silvicultural Decision Making“, Sustainable Forest Management, Springer/ Berlin, Heidelberg, S. 237-262

Schmitz, F.; Polley, H.; Henning, P. u. Schwitzgebel P. (2005): „Die zweite Bundeswaldinventur - BWI“, Broschüre, Forestry Commission/ London, $87 \mathrm{~S}$.

Schober, R. (1975): „Ertragstafeln wichtiger Baumarten“, J.D. Sauerländer's Verlag/ Frankfurt am Main, $154 \mathrm{~S}$.

Schöpfer, W.; Kändler, G. und Stöhr, D. (2003): „Entscheidungshilfen für Forst- und Holzwirtschaft“, Forst und Holz (58/18), S. 545-550

Schröder, J.; Röhle, H. u. Münder, K. (2005): „Simulation und Bewertung von Managementoptionen mit dem Waldwachstumssimulator BWINPro-S“, Forst und Holz (60/10), S. 411-415

Schütz J.P (2001): „Der Plenterwald und weitere Formen strukturierter und gemischter Wälder“, Parey/ Berlin, 207 S.

Schwappach, A. (1889): „Wachsthum und Ertrag normaler Kiefernbestände in der norddeutschen Tiefebene“, Springer/ Berlin, Heidelberg, New York, $72 \mathrm{~S}$.

Schwappach, A. (1890): „Wachstum und Ertrag normaler Fichtenbestände“, Springer/ Berlin, Heidelberg, New York, $100 \mathrm{~S}$.

Schweiger, J. u. Sterba, H. (1997): „A model describing natural regneration recruitment of Norway spruce ((Picea abies ( L.) Karst.) in Austria“, Forest Ecology and Management (97), S. 107-118

Schwoerer, M. (2001): „Deutsche Aktivitäten zur Umsetzung der AGENDA 21“, Forst und Holz (56), S. 463-466

Seibert, P. u. Conrad-Brauner, M. (1995): „Konzept, Kartierung und Anwendung der potentiellen natürlichen Vegetation mit dem Beispiel der PNV-Karte des unteren Inntales“, Tuexenia (15), S. $25-43$

Seifert, S.; Seifert, E.; Biber, P.; Seifert, T.; Klemmt, H.J. u. Pretzsch, H. (2005): „SILVA 3 - eine modulare Softwarelösung“, AFZ/ Der Wald (21), S. 1144-1145

Shannon, C. E. (1948): „The mathematical theory of communication“, The mathematical theory of communication, University of Illinois Press, Urbana, S. 3-91

Shugart, H.H. (1984): „A theory of forest dynamics - the ecological implications of forest succession models“, Springer/ Berlin, Heidelberg, New York, Tokio, 278 S.

Sieber, P. (1928): „Der Dauerwald“, Springer/ Berlin, 110 S.

Sievänen, R. (1993): „A process-based model for the dimensional growth of even-aged stands“, Scandinavian Journal of Forest Research (8), S. 28-48 
Sloboda, B. (1983): „Kolmogorow-Suzuki und die stochastische Differentialgleichung als Beschreibungsmittel der Bestandesevolution", Forest Growth Modeling and Simulation, Mitteilungen der Forstlichen Bundes-Versuchsanstalt Wien (147), S. 71-82

Sloboda, B. u. Saborowski, J. (1977): „Stochastic Differential Equations as growth models, some examples and unsolved problems“, Beiträge zur Zuwachsforschung, Mitteilungen der Forstlichen Bundes-Versuchsanstalt Wien (120/3), S. 69-86

Söderberg, I. u. Ledermann, T. (2003): „Algorithms for simulating thinning and harvesting in five European individual-tree growth simulators: a review“, Computers and electronics in agriculture (39), S. 115140

Sodtke, R.M.; Schmidt, M.; Fabrika, M.; Nagel, J.; Dursky, J. u. Pretzsch, H. (2004): „Anwendung und Einsatz von Elnzelbaummodellen als Komponenten von entscheidungsunterstützenden Systemen für die strategische Forstplanung“, Forstarchiv (75/51-64), S. 51-64

Sodtke, R.M.; Utsching, H. u. Pretzsch, H. (2006): „A Decision Support System for Multi-Criteria Forest Estate Planning, Integrating a Forest Growth Simulator, Fuzzy Inference Techniques and a Heuristic Optimisation Approach“, Sustainable Forest Management, Springer/ Berlin, Heidelberg, S. $211-236$

Speidel, G. (1972): „Planung im Forstbetrieb: Grundlagen und Methoden der Forsteinrichtung“, Parey/ Hamburg, $267 \mathrm{~S}$.

Spellmann, H. (1994a): „Ertragskundliche Aspekte des Fremdländeranbaus“, Allgemeine Forst- und Jagdzeitung (165/2), S. 27-34

Spellmann, H. (1994b): „Auswirkungen von Läuterungseingriffen auf die Schwachholzproduktion“, Forst und Holz (49/11), S. 288-300

Spellmann, H. (1997): „Ertragsentwicklung im „LÖWE“-Wald der Niedersächsischen Landesforstverwaltung“, Forst und Holz (52/24), S. 711-718

Spellmann, H. (2003): „Sicherung einer nachhaltigen Waldentwicklung auf überbetrieblichen Ebenen“, Forstwissenschaftliches Centralblatt (122), S. 250-257

Spellmann, H. (1996): „Waldbau im Wandel“, Forst und Holz (51/1), S. 3-9

Spellmann, H.; Hillebrand, K. u. Cornelius, P. (2001): „Konzept zur Erfassung und Sicherung der Nachhaltigkeit in multifunktional genutzen Wäldern", Forst und Holz (56/15), S. 469-473

Spellmann, H.; Kehr I.; Hillebrand, K.; Schütze, C.; Pretzsch, H.; Pott, M.; Bitter, A. W.; Koffinke, B.; Bredemeier M. u. Schulte-Bisping, H. (2004): „Nachhaltige und multifunktionale Forstwirtschaft", DBU-Bericht, Erich Schmidt Verlag, S. 15-28

Spellmann, H.; Nagel, J.; Böckmann, T. (1999): „Summarische Nutzungsplanung auf der Basis von Betriebsinventurdaten“, Allgemeine Forst- und Jagdzeitung (170/7), S. 122-128

Spiecker, H.; Hansen, J.; Klimo, E.; Skovsgaard, J.P.; Sterba, H. u. von Teuffel, K. (2004): „Norway spruce conversion - options and consequences", European Forest Institute Research Report, European Forest Institute/ Leiden (18), 269 S.

Spiecker, H.; Mielikäinen, K.; Köhl, M. u. Skovsgaard, J.P. (1996): „Growth tends in European forests“, European Forest Institute Research ReportSpringer/ Berlin, Heidelberg, New York (5), $372 \mathrm{~S}$.

Stage, A.R. (1973): „Prognosis model for stand development“, USDA Forest Service research paperUS Gov. Print. Off./ Washington, DC (INT-137), 32 S. 
Sterba, H. (1975): „Assmanns Theorie der Grundflächenhaltung und die „Competition-Density-Rule“ der Japaner Kiro, Ando und Tadaki“, Centralblatt für das gesamte Forstwesen (92/1), S. 46-62

Sterba, H. (1977): „Single stem models from inventory data with temporary plots“Mitteilungen der Forstlichen Bundes-Versuchsanstalt Wien, Beiträge zur Zuwachsforschung (120/3), S. 87-101

Sterba, H. (1981): „Natürlicher Bestockungsgrad und Reinekes SDI“, Centralblatt für das gesamte Forstwesen (98/2), S. 101-116

Sterba, H. (1987): „Estimating potential density from thinning experiments and inventory data“, Forest Science (33/4), S. 1022-1034

Sterba, H. u. Monserud, R. (1995): „Validation of the single stand growth simulator PROGNAUS“, IUFRO World Congress/ Tampere (Finland), S. 36-39

Sterba, H.; Moser, M.; Hasenauer, H. u. Monserud, R.A. (1995): „PROGNAUS - ein abstandsunabhängiger Wachstumssimulator für ungleichaltrige Mischbestände“, Tagungsbericht der Sektion Ertragskunde DVFFA (Eberswalde), Berlin, S. 173-183

Stern, H.; Bibelriether, H.; Burschel, P.; Plochmann, R.; Schröder, W. u. Schulz, H. (1979): „Rettet den Wald“, Kindler/ München, 393 S.

Straubinger, F. (1996): „Naturgemäßer Waldbau“Alternative Konzepte, Ökologische Waldwirtschaft Grundlagen-Aspekte-Beispiele (88) ,Stiftung Ökologie \& Landbau C.F. Müller Verlag, S. 107-114

Sturm, K. (1993): „Prozeßschutz - ein Konzept für naturschutzgerechte Waldwirtschaft", Zeitschrift für Ökologie und Naturschutz (2), S. 181-192

Sturm, K. (1994): „Kurzfassung Greenpeace-Studie - Naturnahe Waldnutzung in Mitteleuropa“, unveröffentlicht/ Greenpeace Deutschland, 48 S.

Sturm, K. (1995a): „Naturnahe Waldnutzung in Mitteleuropa“, Der Dauerwald (12), S. 6-21

Sturm, K. (1995b): „Wald ohne Förster?“, Schriftenreihe für Landschaftspflege und Naturschutz, S. 1-11

Suzuki, T. (1971): „Forest transition as a stochastik process“Mitteilungen der Forstlichen BundesVersuchsanstalt Wien, Beiträge zur Zuwachsforschung (91), S. 69-86

Teegelbekkers, D. (2001): „Bedeutung der paneuropäischen Kriterien und Indikatoren einer nachhaltigen Waldbewirtschftung für den Zertifizierungsprozess“, Forst und Holz (56/15), S. 466-469

Tomlin, C. D. (1990): „Geographic information systems and cartographic modeling“, Englewood Cliffs, NJ, Prentice Hall, 249 S.

Tremer, N. (2004): „Entwicklung von Methoden zur Schätzung des Ist-Zustandes und zur Prognose der Strukturdynamik von Naturverjüngung", Tagungsbericht der Sektion Ertragskunde DVFFA (Stift Schlägl), S. 119-128

Tremer, N.; Hansen, J. u. Schmidt, M. (2005): „Schätzung der Naturverjüngungsstruktur auf Basis von Betriebsinventuren“, Allgemeine Forst- und Jagdzeitung (176/1), S. 1-14

Tüxen, R. (1956): „Die heutige potentielle natürliche Vegetation als Gegenstand der Vegetationskartierung", Angewandte Pflanzensoziologie (13/l), S. 5-42

Ulrich, Hans (2001): „Das St. Galler Management-Modell“, Gesammelte SchriftenHaupt/ Bern, Stuttgart, Wien (Bd. 2), $54 \mathrm{~S}$.

United Nations Conference on Environment and Development (1992): „Report of the United Nations Conference on Environment and Development", Rio de Janeiro, $114 \mathrm{~S}$.

Utschig, H. (2000): „Wachstum vorherrschender Buchen in Abhängigkeit von Standort und Behandlung“, Forst und Holz (55/2), S. 44-50 
Vacik, H.; Lexer, M.J.; Rauscher M.H.; Reynolds, K.M. u. Brooks, R.T. (2003): „Decision support for multiple purpose forestry", CD-Proceedings (IUFRO)

Vacik, H.; Lexer, M.J. u. Englisch, M. (2004): „Einsatz des Decision Support Systems DSD v1.1 zur Unterstützung der forstlichen Beratung im Landesforstdienst", Forstarchiv (75), S. 180-189

Valsta, L. (2002): „Economic evaluation of uneven-aged management“, Managing Forest Ecosystems: Continious Cover Forestry - Assessment, Analysis, Scenarios, Kluwer Academic Publishers/ Dordrecht, Boston, London, S. 281-292

van Deusen, P.C. u. Biging, G.S. (1985): „STAG a stand generator for mixed species stands. Version 2.0 Res Note 11", Northern California Forest Yield Cooperative, Department of Forestry and Research Management, University of California/ Berkeley, $25 \mathrm{~S}$.

van Laar, A.; Rois Diaz, M. u. von Gadow, K. (2003): „Zum Jugendwachstum in einem Buchenmischbestand“, Allgemeine Forst- und Jagdzeitung (174/7), S. 131-136

Vanselow, K. (1949): „Der bayerische Femelschlag und seine Fortbildung“, Forstwissenschaftliche Praxis, Bayrischer Landwirtschaftsverlag $\mathrm{GmbH} / \mathrm{München} \mathrm{(6),} 56 \mathrm{~S}$.

Verein für forstliche Standortskunde und Forstpflanzenzüchtung (2005): „Waldökologische Naturräume Deutschlands - Forstliche Wuchsgebiete und Wuchsbezirke", Mitteilungen des Vereins für forstliche Standortskunde und Forstpflanzenzüchtung (43), 324 S.

Verein Naturschutzpark e.V. (1997): „Naturschutzgebiet Lüneburger Heide, Geschichte - Ökologie Naturschutz", Schriftenreihe des Vereins Naturschutzpark e.V., H.M. Hauschild GmbH/ Bremen, $367 \mathrm{~S}$.

von Gadow, K. (1987): „Untersuchungen zur Konstruktion von Wuchsmodellen für schnellwüchsige Plantagenbaumarten“, Forstliche Forschungsberichte München (77), 147 S.

von Gadow, K. (2004): „Forsteinrichtung als Forschungsfeld - lohnend und unverzichtbar“, Allgemeine Forst- und Jagdzeitung (175/7-8), S. 134-141

von Gadow, K. u. Hui, G.Y (1999): „Modeling forest development“, Kluwer/ Dordrecht, 213 S.

von Goethe, J.W. (1814): „Aus meinem Leben: Dichtung und Wahrheit“, „Es ist dafür gesorgt, dass die Bäume nicht in den Himmel wachsen", Cotta/ Tübingen (Theil 3), 538 S.

von Guttenberg, A.R. (1915): „Wachstum und Ertrag der Fichte im Hochgebirge“, Verlag Deuticke/ Wien, Leipzig, $153 \mathrm{~S}$.

von Lüpke, B. (2004): „Risikominderung durch Mischwälder und naturnaher Waldbau: ein Spannungsfeld“, Forstarchiv (75/43-50), S. 43-50

von Lüpke, B. u. Hauskeller-Bullerjahn, K. (2004): „Beitrag zur Modellierung der Jungwuchsentwicklung am Beispiel von Traubeneichen-Buchen-Mischverjüngungen“, Allgemeine Forst- und Jagdzeitung (175/4-5), S. 61-69

von Teuffel, K.; Hein, S.; Kotar, M.; Preuhsler, E.P.; Puumalainen, J. u. Weinfurter, P. (2006): „End User Needs and Requirements“, Sustainable Forest Management, Springer/ Berlin, Heidelberg, S. 1938

Wagner, C. (1912): „Der Blendersaumschlag und sein System“, H. Laupp/ Tübingen, 368 S.

Wagner, S. (2004a): „Möglichkeiten und Beschränkungen eines funktionsorientierten Waldbaus“, Forst und Holz (59/3), S. 105-111

Wagner, S. (2004b): „Klimawandel - einige Überlegungen zu waldbaulichen Strategien“, Forst und Holz (59/8), S. 394-398 
Watt, A.S. (1925): „On the Ecology of British Beechwoods with Special Reference to Their Regeneration: Part II, Sections II and III. The Development and Structure of Beech Communities on the Sussex Downs“, Journal of Ecology (13/1), S. 27-73

Watt, A.S. (1947): „Pattern and process in the plant community“, Journal of Ecology (35/1-2), S. 1-22 Weimann, H.J (2003): „Wurzeln der Nachhaltigkeit“, Forst und Holz (58/6), S. 153-155

Wenk, G.; Antanaitis, V.; Smelko, S. (1990): „Waldertragslehre“, Deutscher Landwirtschaftsverlag, 448 S.

Wenk, G.; Römisch, K. u. Gerold, D. (1985): „DDR-Fichtenertragstafel 1984“, Agrarwissenschaftliche Gesellschaft der DDR/ Dresden, $64 \mathrm{~S}$.

Wensel, L.C. u. Daugherty, P.J. (1984): „CACTOS users guide: the California conifer timber output simulator", Northern California Forest Yield Cooperative Res Notes, Department of Forestry Research and Management, University of California/ Berkeley (10), $91 \mathrm{~S}$.

Wensel, L.C. u. Koehler, J.R. (1985): „A tree growth projection system for northern California coniferous forests, Res. note 12", Northern California Forest Yield Cooperative, Department of Forestry and Research Management, University of California/ Berkeley, $30 \mathrm{~S}$.

Westphal. C. (2000): „Der Naturwald 'Ehrhorner Dünen' und die angrenzenden Kiefernbestände im Wirtschaftswald“NUA Seminarbericht (4), S. 295-297

Wiedemann, E. (1926): „Die zweckmäßige Breite der Kahlschläge im Kiefernwald“, Zeitschrift für Forstund Jagdwesen (58), S. 333-350

Wiedemann, E. (1942): „Der gleichaltrige Fichten-Buchen-Mischbestand“, Mitteilungen aus Forstwirtschaft und Forstwissenschaft, S. 1-88

Wiedemann, E. (1949): „Ertragstafeln der wichtigen Holzarten bei verschiedener Durchforstung“, Schaper/ Hannover, $99 \mathrm{~S}$.

Wikipedia (2006a) „Management“, http://de.wikipedia.org/wiki/Management, 15.06.2006

Wikipedia (2006b): „Strategie“, http://de.wikipedia.org/wiki/Strategie, 15.06.2006

Wikipedia (2006c): „Unternehmensstrategie“, http://de.wikipedia.org/wiki/Unternehmensstrategie, 15.06.2006

Wild, R. (1995): „Operationalität eines Verjüngungsaufnahmeverfahrens“, Diplomarbeit, Universität für Bodenkultur/ Wien, $97 \mathrm{~S}$.

Wilhelm, G.J.; Letter, H.A. u. Eder, W. (1999): „Konzeption einer naturnahen Erzeugung von starkem Wertholz - Die Phase der Qualifizierung", AFZ/ Der Wald (5), S. 234-236

Wilhelm, G.J.; Letter, H.A. u. Eder, W. (1999a): „Konzeption einer naturnahen Erzeugung von starkem Wertholz", AFZ/ Der Wald (5), S. 232-240

Wilhelm, G.J.; Letter, H.A. u. Eder, W. (1999b): „Konzeption einer naturnahen Erzeugung von starkem Wertholz - Die Phase der Reife“, AFZ/ Der Wald (5), S. 239-240

Wilhelm, G.J.; Letter, H.A. u. Eder, W. (1999c): „Konzeption einer naturnahen Erzeugung von starkem Wertholz - Zielsetzungen und Prinzipien“, AFZ/ Der Wald (5), S. 232-233

Wilhelm, G.J.; Letter, H.A. u. Eder, W. (1999d): „Konzeption einer naturnahen Erzeugung von starkem Wertholz - Die Phase der Dimensionierung", AFZ/ Der Wald (5), S. 234-238

Windhager, M. (1999): „Evaluierung von verschiedenen Waldwachstumssimulaltoren“ (Dissertation), 210 S.

Wobst, W. (1950): „Arbeitsgemeinschaft „Naturgemäße Waldwirtschaft““”, AFZ (5/8), S. 1 
Wollborn, P. (1998): „Waldbauliche Steuerung, Nachhaltigkeitskontrolle und Verbesserung des Betriebsergebnisses in strukturreichen Wäldern“, Der Dauerwald (18), S. 8-28

Wollborn, P. (2000): „Ist weniger mehr? Gedanken zu Ergebnissen und betriebswirtschaftlichen Auswirkungen einer naturnahen Waldwirtschaft in der Niedersächsischen Landesforstverwaltung“, Forst und Holz (55/7), S. 202-207

Wollborn, P. (2004): „Vorteile naturgemäßer Waldwirtschaft für die Niedersächsische Landesforstverwaltung", Forst und Holz (59/1), S. 16-21

Wollborn, P. u. Böckmann, T. (1998): „Ein praktikables Modell zur Strukturierung des Vorrats aus Ertragstafelschätzung“, Forst und Holz (53/18), S. 547-550

World Commission of Environment and Development (1987): „Our common future“, Report of the world Commission of Environment and Development ('Brundtland Report), http://en.wikipedia.org/wiki/World_Commission_on_Environment_and_Development, 15.06.2006

Wykoff, W.R.; Crookston, N.L. u. Stage, A.R. (1982): „User's guide to the Stand Prognosis Model“, General technical ReportUSDA Forest Service/ Jakobsen

Washington, DC (INT-133), $111 \mathrm{~S}$.

Zerbe, S. (1997): „Stellt die potentielle natürliche Vegetation (PNV) eine sinnvolle Zielvorstellung für den naturnahen Waldbau dar?", Forstwissenschaftliches Centralblatt (116), S. 1-15

Zerbe, S. u. Kempa, D. (2005): „Waldumbau und Biodiversität - Unterschiedliche forstliche Entwicklungsziele und deren Auswirkungen auf die biologische Vielfalt", Naturschutz und Landschaftsplanung (37/4), S. 106-114

Zingg, A. (2003): „Dauerwald - ein neues altes Thema der Waldwachstumsforschung“, Informationsblatt Forschungsbericht Wald/Eidgenössische Forschungsanstalt WSL (15), S. 1-3

Zutter, B.R.; Oderwald, R.G.; Murphy, P.A. u. Farrar, J.R.R.M. (1986): „Characterizing diameter distributions with modified data types and forms of the Weibull distribution“, Forest Science (32/1), S. $37-48$ 


\section{Abbildungen}

Abbildung 1.2.a: Elemente einer Entscheidungssituation (aus Oesten und Roeder 2002).

Abbildung 2.2.2.a: Einordnung eines Waldbaukonzepts im Zusammenhang von forstpolitischen Zielvorgaben, Betriebspolitik, strategischem und operativem Management am Beispiel der Landesforstverwaltung Rheinland-Pfalz (nach Oesten und Roeder 2002).

Abbildung 2.3.1.a: Von ökophysiologisch basierten Prozeßmodellen über Managementmodelle bis zu Sukzessions- und Biommodellen nimmt die räumliche und zeitliche Aggregation bei der Nachbildung der Prozesse und Strukturen zu (nach Pretzsch 2001).

Abbildung 2.3.1.b: Simulationsprozeß bei positionsabhängigen Einzelbaummodellen in schematischer Darstellung (nach Ek und Dudek 1980 in Pretzsch 2001)

Abbildung 2.3.2.a: System mit den Ebenen Bestand und Baum, den externen Variablen Struktureingriff und Standort und der Rückkopplungsschleife Bestandestruktur $\rightarrow$ Zuwachs $\rightarrow$ Baumzustand $\rightarrow$ Bestandesstruktur (nach Pretzsch 2001)...

Abbildung 3.1.2.a: Parameter für die Ableitung der potentiellen natürlichen Vegetation eines bestimmten Zeitraums (aus Kaiser 1996). 44

Abbildung 3.2.1.a: Das Untersuchungsgebiet Lüneburger Heide mit getrennt ausgewiesenen Besitzarten.

Abbildung 3.2.1.b: Flächenanteile der Bestandestypengruppen im Untersuchungsgebiet. .53

Abbildung 3.2.1.c: Vorratsstruktur im Untersuchungsgebiet des EU-Life Projekts „Demonstration of Methods to Monitor Sustainable Forestry - Nachhaltige multifunktionale Waldentwicklung“, nach Altersklassen in Prozent des Gesamtvorrats (nach NFV und NFA 2002). 54

Abbildung 3.3.2.a: Datenbankaufbau der Simulation. Der Aufbau der Ergebnisdatenbank kann optional gesteuert werden. Der Benutzer kann individuell wählen, welche Zwischenergebnis- und Endergebnistabellen er festhalten möchte.

Abbildung 3.3.2.b: Übersicht über die Verarbeitungsschritte: (1) Datenvorbereitung und Ausgabe der Ausgangssituation, (2) szenarienabhängige Fortschreibung und Ausgabe der

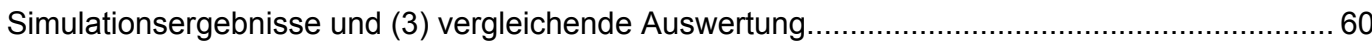

Abbildung 3.5.a: Ablauf der szenarienabhängigen Fortschreibung des Untersuchungsgebiets.................69

Abbildung 3.6.2.a: Erntekostenfreie Holzerlöse13 für Bäume der Wertklasse II.......................................8 88

Abbildung 3.6.2.b: Erntekostenfreie Holzerlöse13 für Bäume der Wertklasse III.......................................89

Abbildung 4.1.1.a: Simulationsergebnisse der Szenarien LÖWE, PNV, ERTRAG und PROZESS nach vierzigjähriger Entwicklung eines Bestandes aus fünfzigjähriger Kiefer (Bestand 1 aus Tabelle 3.2.2.a)

Abbildung 4.1.1.b: Die vierzigjährige Entwicklung eines Bestandes aus fünfzigjähriger Quo und der Zwischenergebnisse der Szenarien LÖWE, PNV, ERTRAG und (auch für die Strategien LÖWE und PNV, die zwei Eingriffe im Jahrzehnt vorsehen). Stämme werden mit einer dreifach vergrößerten Grundfläche dargestellt. 98

Abbildung 4.1.1.c: Simulationsergebnisse der Szenarien LÖWE, PNV, ERTRAG und PROZESS nach vierzigjähriger Entwicklung eines Bestandes aus dreißigjähriger Fichte (Bestand 2 aus Tabelle 3.2.2.a)........ 
Abbildung 4.1.1.d: Simulationsergebnisse der Szenarien LÖWE, PNV, ERTRAG und PROZESS nach vierzigjähriger Entwicklung eines Bestandes aus fünfzigjähriger Kiefer und dreißigjähriger Fichte (Bestand 3 aus Tabelle 3.2.2.a) 107

Abbildung 4.1.2.a: Simulationsergebnisse der Szenarien LÖWE, PNV, ERTRAG und PROZESS nach vierzigjähriger Entwicklung eines Bestandes aus hundertzehnjähriger Kiefer (Bestand 4 aus Tabelle 3.2.2.a) 110

Abbildung 4.1.2.b: Die vierzigjährige Entwicklung eines Bestandes aus Stammverteilungsplänen des Status-Quo und der Zwischenergebnisse der Szenarien Jahren festgehalten worden (auch für die Strategien LÖWE und PNV, die zwei Grundfläche dargestellt. 112

Abbildung 4.2.1.a: Prozentualer Volumenanteil der BHD-Klassen am Gesamtvorrat des Untersuchungsgebiets. 119

Abbildung 4.3.1.a: Prozentualer Volumenanteil der BHD-Klassen am Gesamtvorrat des Untersuchungsgebiets nach der vierzigjährigen Simulation der Variante LÖWE im Vergleich zum Status Quo

Abbildung 4.3.1.b: Prozentualer Volumenanteil der BHD-Klassen am Gesamtvorrat des Untersuchungsgebiets nach der vierzigjährigen Simulation der Variante PNV im Vergleich zum Status Quo... 123

Abbildung 4.3.1.c: Prozentualer Volumenanteil der BHD-Klassen am Gesamtvorrat des Untersuchungsgebiets nach der vierzigjährigen Simulation der Variante ERTRAG im Vergleich zum Status Quo. 123

Abbildung 4.3.1.d: Prozentualer Volumenanteil der BHD-Klassen am Gesamtvorrat des Untersuchungsgebiets nach der vierzigjährigen Simulation der Variante PROZESS im Vergleich zum Status Quo.

Abbildung 4.3.4.a: Verteilung der Zieltypenanteile der drei Varianten (LÖWE, PNV und ERTRAG) an der Fläche des Untersuchungsgebiets. Da in der Variante PROZESS keine keine Zieltypen angestrebt werden, können für diese Variante keine Anteile angegeben werden. 130

Abbildung 4.3.4.b: Verteilung der Bestandestypenanteile an der Fläche des Untersuchungsgebiets zum Anfang der Simulation (Status Quo) und am Ende der Simulationen der vier Varianten (LÖWE, PNV, ERTRAG und PROZESS) über einen vierzigjährigen Simulationszeitraum.........................131

Abbildung 4.3.5.a: Vergleichende Übersicht ausgewählter Auswertungsparameter des Untersuchungsgebiets nach der vierzigjährigen Simulation der Varianten LÖWE, PNV, ERTRAG und PROZESS. Die Achsen der einzelnen Parameter bezeichnen mit 100\% den jeweils höchsten vorkommenden Wert der Simulationsläufe. Die Datenpunkte geben an, wieviel Prozent dieses Werts in der jeweiligen Variante erreicht wurden. 134 


\section{Tabellen}

Tabelle 3.2.1.a: Klimadaten des Wuchsbezirks „Hohe Heide“ im Vergleich zu benachbarten Wuchsbezirken (zum Teil aus Verein für forstliche Standortskunde und Forstpflanzenzüchtung 2005) 50

Tabelle 3.2.2.a: Übersicht über die Eingangswerte für den Aufbau der vier Beispielbestände...................56

Tabelle 3.4.1.a: Aufbau der Tabelle für Forsteinrichtungsdaten aus der Quelldatenbank......................... 62

Tabelle 3.5.a: Rahmenwerte (bestandesbezogene Werte) zu den Strategien LÖWE, PNV, ERTRAG und

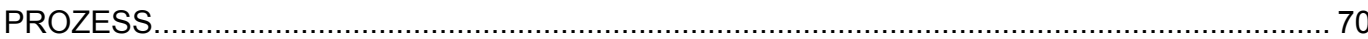

Tabelle 3.5.b: Rahmenwerte (artenbezogene Werte) zu den Strategien LÖWE, PNV, ERTRAG und

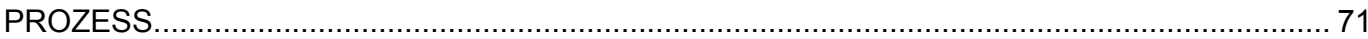

Tabelle 3.5.c: Verwendete Maßnahmenelemente und ihre Zuordnung zu den Kategorien Schutz, Endnutzung, Durchforstung und Verjüngung... 72

Tabelle 3.6.2.a: Übersicht über die Auswertungsparameter der Simulation zu den Kriterien „forstliche Ressourcen“, „Produktionsfunktion“, „Biologische Diversität“ und „Zielerreichung“.... 87

Tabelle 3.6.2.b: Pflanzungskosten und Pflanzungskostengrundlage (Stückzahlen, Pfanzenkosten, Pflanzkosten, Kosten für Bodenvorbereitung und Zaunbau) für die Baumartengruppen Eiche, Buche, ALh, ALn, Fichte, Douglasie und Kiefer. 90

Tabelle 4.1.1.a: Übersicht über die Ergebnisse der Simulation des Bestandes 1 anhand der Auswertungsparameter. 100

Tabelle 4.1.1.b: Übersicht über die Ergebnisse der Simulation des Bestandes 2 anhand der Auswertungsparameter. 106

Tabelle 4.1.1.c: Übersicht über die Ergebnisse der Simulation des Bestandes 3 anhand der Auswertungsparameter. 108

Tabelle 4.1.2.a: Übersicht über die Ergebnisse der Simulation des Bestandes 4 anhand der Auswertungsparameter. 117

Tabelle 4.2.3.a: Zielerreichungsprozent zu Beginn der Simulation für die Varianten LÖWE, PNV und ERTRAG. In der Variante PROZESS wird kein Ziel zur Baumartenzusammensetzung angestrebt, daher kann für sie keine Angabe gemacht werden. 120

Tabelle 4.3.a: Übersicht über die Ergebnisse der Simulation anhand der Auswertungsparameter. 121

Tabelle 5.2.3.a: Kostenzuschläge und Kostenabschläge im Bereich der Holzernte für die Strategien LÖWE, PNV, ERTRAG und PROZESS. 


\section{Glossar}

Werden in einer Definition Begriffe verwendet, die an anderer Stelle des Glossars erklärt werden, sind sie mit einem Stern* gekennzeichnet.

Absolute Bonität: „Die absolute Höhenbonität gibt an, welche Bestandesmittelhöhe oder Oberhöhe* ein Bestand* im Alter von 100 Jahren erreicht hat." (KRAMER UND AKCA 1982, S. 194)

Abteilung: „Dauerhafte Einheiten der Waldeinteilung. Sie dienen der räumlichen Orientierung im Walde." (Niedersächsische Landesforstverwaltung 1987, S. 4 A)

Abtriebswert: Erntekostenfreier Holzerlös für den Fall, daß alle Bäume einer Bestandesfläche in einem Hiebsvorgang zum betrachteten Zeitpunkt geschlagen werden. (OESTEN UND ROEDER 2002)

Andere Laubbäume mit hoher Umtriebszeit: „Rüster, Ahorn, Esche, Kirsche, Linde, Elsbeere, Nußbaum, Eßkastanie, Robinie u.a." (Niedersächsische Landesforstverwaltung 1987, S. 6 A)

Andere Laubbäume mit niedriger Umtriebszeit: „sämtliche Birken-, Erlen-, Weiden- und Pappelarten, Eberesche, Traubenkirsche u.a." (Niedersächsische LandesforstVerWaltung 1987, S. 6 A)

ALh: siehe „Andere Laubbäume mit hoher Umtriebszeit“*

ALn: siehe „Andere Laubbäume mit niedriger Umtriebszeit“*

Altersklasse: „Bei der zahlenmäßigen Darstellung werden die einzelnen Bestände oder Bestandesteile zwanzigjährigen Altersklassen zugeteilt. Die Altersklassen werden bei der jüngsten beginnend mit römischen Ziffern bezeichnet ( $I=20$, II = 21-40 usw.)." (KRAmer 1976).

Ästung: „Pflegliches Absägen oder Abhauen von Zweigen stehender Bäume.“ (BonnemanN 1967)

A-Wert: Maß für die Konkurrenz zwischen zwei Bäumen (siehe Johann 1982 und Johann 1983).

Behandlungszyklus: In dieser Arbeit wird unter einem Behandlungszyklus der Ablauf des Simulationsprogramms verstanden. Der szenarienabhängige Behandlungszyklus besteht aus Einlesen der Daten, Fortschreibezyklus* und Ausgabe der Daten. Siehe auch Seiten $67 \mathrm{ff}$.

Bestand: „Waldbaulich selbständiger Waldteil, der auf Grund seiner Größe und Form die kleinste Einheit des waldbaulichen Handelns für einen längeren Zeitraum darstellt.“ (BonNemann 1967)

Bestandesaufbau: „Kennzeichnung eines Bestandes* nach seiner Art und seiner Form. Dabei wird die Art nach folgenden Merkmalen beschrieben: 1. Baumarten, 2. Alterszusammensetzung <...>. Die Form kann durch folgende Merkmale beschrieben werden: 1. Verteilung der einzelnen Baumarten $<\ldots>$, 2. Vertikaler Aufbau <...>." (BONNEMANn 1967)

Bestandesschicht: Die dem Waldwachstumsmodell zugrunde liegende Höhenschichteneinteilung lehnt sich an den oberhöhenabhängigen Ansatz von Assmann (nach Assmann 1961, S. 82-99) an, der Höhenschichten ungleicher relativer Mächtigkeit ausweist, die annähernd zum abnehmenden Lichtgenuß proportional gestaffelt sind: 
- Oberschicht: Bäume mit einer Höhe von über $80 \%$ der Oberhöhe*

- Mittelschicht: Bäume mit einer Höhe von über 50\% - 80\% der Oberhöhe

- Unterschicht: Bäume mit einer Höhe von 0\% - 50\% der Oberhöhe

Die Einteilung nach der Bestandesoberhöhe ist dahingehend modifiziert, daß bestimmte absolute Oberhöhen überhaupt erst erreicht werden müssen, um die Ausweisung von mehrschichtigen Beständen zuzulassen. Konkret wurde folgendes festgelegt:

- Drei Höhenschichten können ausgewiesen werden bei einer Oberhöhe von über $18 \mathrm{~m}$

- Zwei Höhenschichten können ausgewiesen werden bei einer Oberhöhe von 13m - 18m

- Eine Höhenschicht wird ausgewiesen bei einer Oberhöhe von unter $13 \mathrm{~m}$

Diese absoluten Mindesthöhen entsprechen weitestgehend den Vorgaben in den Merkblättern der Niedersächsischen Landesforstverwaltung (NiedersäCHSISCHE LANDESFORSTVERWALTUNG 2006) zur Unterscheidung von Baum- und Stangenholz.

Bestandestyp: „Zusammenfassung von Beständen mit gleicher oder ähnlicher Baumartenzusammensetzung." (NIEDERSÄCHSISCHE LANDESFORSTVERWALTUNG 1987, S. 9-11 A) Ein Bestandestyp setzt sich in dieser Arbeit aus Haupt- und Nebenbaumarten* nach der Definition des Programms BWINPro zusammen. Die Bezeichnungen sind der Definition der Veröffentlichung der Niedersächsischen Landesforstverwaltung (NIEDERSÄCHSISCHE LANDESFORSTVERWALTUNG 1987) entnommen.

Bestandestypengruppe: Eine Bestandestypengruppe faßt mehrere ähnliche Bestandestypen* zusammen. In dieser Arbeit wird zwischen Eichen-Bestandestypen, BuchenBestandestypen, ALh*-Bestandestypen, ALn*-Bestandestypen, Fichten-Bestandestypen, Douglasien- Bestandestypen und Kiefern-Bestandestypen unterschieden. In einigen Fällen werden ALh*-Bestandestypen und ALn*-Bestandestypen zu einer Gruppe zusammengefaßt.

Betriebswerk: „Waldzustandserhebung sowie Ergebnisniederschrift und Handlungsanweisung über forstbetriebliche Maßnahmen des abgelaufenen und bevorstehenden Betriebsregelungszeitraumes." (NFV UND NFA 2002)

Bestockung: „Im gebräuchlichen Sinne: Baumbestand einer Waldfläche.“ (BonNemann 1967)

Bestockungsgrad: „Verhältnis der tatsächlichen Grundfläche* je ha einer Bestandesschicht* eines Bestandes zu der entsprechenden Grundfläche der Ertragstafel i.d.R. mäßige Durchforstung." (Niedersächsische LandesforstVerWaltung 1987, S. 12A)

BHD: siehe „Brusthöhendurchmesser“*

Brusthöhendurchmesser: Durchmesser des Baums in 1,30m Höhe

Derbholz: „Derbholz ist die oberirdische Holzmasse über 7cm D.m.R.“ (Durchmesser mit Rinde) (KRAmer 1976)

Dg: siehe „Mitteldurchmesser“*

Dmax: Durchmesser des stärksten Baums (DöbBELER ET AL. 2003)

Durchmischungskoeffizient: Ausgabeparameter der Simulation. Definition siehe Seite $86 \mathrm{ff}$.

Eingriffsturnus: Wird in dieser Arbeit definiert als der Abstand, in dem die Notwendigkeit von forstlichen Eingriffen geprüft wird und gegebenenfalls in den Bestand* eingegriffen wird. Der Turnus ist von der gewählten Waldbaustrategie abhängig. 
Endnutzungsmasse: In dieser Arbeit definiert als summarisches Volumen der Bäume, die innerhalb eines Eingriffs entnommen wurden, der durch ein Maßnahmenelement* des Typs „Endnutzung“ definiert ist.

Endnutzungszeitraum: In dieser Arbeit steuert der Rahmenwert* „Endnutzungszeitraum“, in welchem Zeitraum die Nutzung der Schicht* beim Einsatz des Maßnahmenelementes* „schichtenweise Nutzung“ abzuschließen ist. Der Endnutzungszeitraum beginnt mit dem ersten Endnutzungseingriff. Am Ende des Endnutzungszeitraums steht die vollständige Nutzung der betrachteten Schicht*

Erntefestmeter: „Maßeinheit für Planung, Einschlag, Verkauf und Buchung des Holzes in Kubikmetern Derbholz* ohne Rinde." (Niedersächsische LandesforstVerWaltung 1987, S. 15 A)

Erntekostenfreie Holzerlöse: Erträge aus dem Verkauf des Holzes abzüglich der Kosten für Aufarbeitung und Rückung des Holzes. Ausgabeparameter der Simulation. Definition siehe Seite $86 \mathrm{ff}$.

Erntereife Bestandesschicht: In dieser Arbeit definiert als Bestandesschicht* (nach Definition von BWINPro), deren $\mathrm{Dg}^{*}$ den Ziel-Dg erreicht hat.

Ertragsklasse: „Relativer Maßstab für die Wuchsleistung eines Bestandes. Sie wird in römischen Ziffern angegeben, wobei I die höchste Leistung angibt. Die Ertragsklasse wird über Bestandeshöhe und -alter der Baumart aus der Ertragstafel abgelesen. In der niedersächsischen Forsteinrichtung* wird statt der Ertragsklasse die Leistungsklasse* verwendet." (NiedersäChsische LandesforstVerWaltung 1987, S. 15 A)

Fm: siehe „Erntefestmeter“*

Freistellungsgrad: Der Freistellungsgrad eines zu pflegenden Baums ist definiert durch die Größe des freien Raumes, der seine Krone nach dem Eingriff umgeben soll.

Forsteinrichtung: „Mittelfristige, periodische Planung im Forstbetrieb. In zehnjährigen Abständen wird der Zustand des Waldes erfasst und darauf aufbauend eine Planung für die nächsten zehn Jahre erstellt. Diese Planung bezieht sich auf jeden einzelnen Bestand $^{*}$ sowie auf den gesamten Forstbetrieb. Neben der forstbetrieblichen Planung umfasst die Forsteinrichtung auch die Planung der Pflege und Entwicklung der für den Naturschutz bedeutsamen Bereiche des Waldes. Ergebnis der Forsteinrichtung ist das Betriebswerk*." (NFV UND NFA 2002)

Fortschreibezyklus: Innerhalb des Fortschreibezyklus wird die Notwendigkeit zum Ausführen eines Maßnahmenelementes* der Maßnahmenkette* geprüft. Nicht alle Maßnahmenelemente* werden zwangsläufig in jedem Fortschreibezyklus ausgeführt. Der Fortschreibezyklus ist Teil des Behandlungszyklus*. (Siehe Seiten $67 \mathrm{ff}$.)

Grundfläche: „Summe der Baumquerschnittsflächen in 1,30m Höhe je ha.“ (BonNEMANN 1967)

Habitatbaum: Habitatbäume sind dazu vorgesehen, in ihrer Alterungs- und Zerfallsphase den Lebensraum von spezialisierten Tier- und Pflanzengruppen zu sichern (unter anderem als stehendes Totholz). Definition im Sinne von BWINPro siehe Seite 72.

Hauptbaumart: „Baumarten eines Bestandes*, auf welche die Bewirtschaftung hauptsächlich abgestellt ist." (Niedersächsische Landesforstverwaltung 1987, S. 20A). Innerhalb des Programms BWINPro wird die Baumart, die den höchsten Kronenschirmflächenanteil des Bestandes* ausmacht, als Hauptbaumart bezeichnet. 
Hg: siehe „Mittelhöhe“*

Hilfsfläche: Ein „Bestand*, der die Kriterien einer Unterabteilung* erfüllt, jedoch in der Regel unter 0,5ha groß ist oder als über 0,5ha große Teilfläche eine kartenmäßig nicht darstellbare Flächenform oder sich verwischende Bestandesunterschiede hat." (Niedersächsische LandesforstVerWaltung 1987, S. 20 A)

Hilfstafeln: Hilfstafeln liegen Informationen aus Ertragstafeln zugrunde. In der Veröffentlichung NiedersÄCHSIsche LANDESFORSTVERWALtung 1987 sind die verwendeten Ertragstafeln namentlich genannt.

Holzboden: Summe aller bestockten Waldflächen eines Forstbetriebs. (siehe NFV UND NFA 2002)

Holzproduktionswert: Ausgabeparameter der Simulation. Definition siehe Seite $86 \mathrm{ff}$.

Index-A: Ausgabeparameter der Simulation. Definition siehe Seite $86 \mathrm{ff}$.

H100: siehe „Oberhöhe“*

Kapitalumschlag: Kapitalumschlag ist das Kapital dividiert durch den Umsatz pro Jahr. (SPEIDEL 1984)

Leistungsklasse: „Der maximale durchschnittliche Gesamtzuwachs der Baumart in Vorratsfestmetern* Derbholz* je Jahr und ha (dGZ max.). Im Anhalt an die Ertragstafeln ist die Leistungsklasse i.d.R. ab $8 \mathrm{~m}$ Bestandeshöhe über Messung von Baumhöhen bestimmt, sonst geschätzt im Anhalt an vergleichbare Bestände“ (NiedersäCHSISCHE LANDESFORSTVERWALtUNG 1987, S. 23 A)

Maßnahmenelement: Ein Maßnahmenelement ist in dieser Arbeit als Teil einer Maßnahmenkette* definiert. Sie lassen sich gemäß den Schritten des Fortschreibezyklus* einem der folgenden Typen zuordnen: Schutz, Endnutzung, Durchforstung oder Verjüngung (Siehe Seiten $67 \mathrm{ff}$.)

Maßnahmenkette: In einer Maßnahmenkette werden in dieser Arbeit die Maßnahmenelemente* in eine Reihenfolge gebracht, die die Priorität ihrer Anwendung zum Ausdruck bringt. Innerhalb des Fortschreibezyklus* wird die Maßnahmenkette* angesteuert.

Maximales/minimales Durchforstungsvolumen: Im Simulationsablauf wird nur dann ein Vornutzungseingriff durchgeführt, wenn auf Bestandesebene eine gewisse Mindestmasse den Vornutzungsbedingungen entspricht (zum Beispiel Bedränger eines Z-Baums oder kein Habitatbaum*). Die Vornutzungsroutine bricht ab, wenn alle zur Endnutzung vorgesehenen Bäume entnommen wurden oder das maximale Vornutzungsvolumen überschritten wird. Beide Grenzen können als Rahmenwert* szenarienabhängig festgelegt werden.

Maximales/minimales Endnutzungsvolumen: Im Simulationsablauf wird nur dann ein Endnutzungseingriff durchgeführt, wenn auf Bestandesebene eine gewisse Mindestmasse den Endnutzungsbedingungen entspricht (zum Beispiel Hiebsreif oder kein Habitatbaum*). Die Endnutzungsroutine bricht ab, wenn alle zur Endnutzung vorgesehenen Bäume entnommen wurden oder das maximale Endnutzungsvolumen überschritten wird. Beide Grenzen können als Rahmenwert* szenarienabhängig festgelegt werden. 
Maximales/minimales Eingriffsvolumen: Im Simulationsablauf wird nur dann ein Vor- oder Endnutzungseingriff durchgeführt, wenn auf Bestandesebene eine gewisse Mindestmasse den Vor- oder Endnutzungsbedingungen entspricht. Die Vor- und Endnutzungsroutinen brechen ab, wenn alle zur Vor- oder Endnutzung vorgesehenen Bäume entnommen wurden oder das maximale Eingriffsvolumen überschritten wird. Beide Grenzen können als Rahmenwert* szenarienabhängig festgelegt werden.

Mitteldurchmesser: Durchmesser des Grundflächenmittelstamms. (KRAMER UND AKCA 1982, S. 126)

Mittelhöhe: „Höhe des Grundflächenmittelstammes.“ (KRAmeR Und AKCA 1982, S. 126)

Naturverjüngung: „Natürliche Neubegründung eines Bestandes* durch Aufschlag, Anflug oder Ausschlag." (Niedersächsische Landesforstverwaltung 1987, S. 26 A)

Nebenbaumart: „Baumarten, auf der nicht das Hauptgewicht der Bewirtschaftung des Bestandes* liegt." (Niedersächsische Landesforstverwaltung 1987, S. 26 A). Innerhalb des Programms BWINPro werden alle Baumarten des Bestandes*, die nicht als Hauptbaumart* gelten, aber einen Kronenschirmflächenanteil von mindestens $10 \%$ des Bestandes* einnehmen, als Nebenbaumart bezeichnet.

Nichtholzboden: Summe aller unbestockten Waldflächen eines Forstbetriebs. „Als unbestockte Waldflächen gelten auch Wege, Schneisen, Leitungstrassen, Wasserläufe und Gräben bis zu 5m Breite sowie sonstige Flächen von unwesentlicher Größe." (NFV UND NFA 2002)

Oberhöhe: „Grundflächenmittelhöhe der 100 stärksten Stämme je ha (h100)." (NIEDERSÄCHSISCHE LANDESFORSTVERWALTUNG 1987, S. 27 A).

Pflanzfläche: Ausgabeparameter der Simulation. Definition siehe Seite $86 \mathrm{ff}$.

pnV: Potentielle natürliche Vegetation

Rahmenwert: Die Rahmenwerte steuern, welche Eingriffe wie stattfinden, und beeinflussen somit den gesamten Simulationsablauf der Waldbaustrategie. Ob ein Maßnahmenelement ${ }^{*}$, das Teil einer Waldbaustrategie ist, zum Einsatz kommt und wie es parametrisiert wird, hängt von der aktuellen Bestandessituation und den szenarienabhängigen Rahmenwerten ab. Es werden Rahmenwerte auf Bestandesebene von Rahmenwerten auf Artenebene unterschieden. Siehe auch Seiten $67 \mathrm{ff}$.

Samenbäume: Als Samenbäume werden in dieser Arbeit Bäume bezeichnet, die nach Hiebsreife im Bestand* verbleiben, um die Verjüngung ihrer Art zu sichern.

Schicht: Siehe „Bestandesschicht“*.

Standort: "Gesamtheit der Umwelteinflüsse in einem Wald, im wesentlichen durch Klima, Boden und Exposition bedingt." (Bonnemann 1967)

Standortskennziffer: Die in dieser Arbeit verwendete Standortskennziffer setzt sich aus Wasserhaushaltskennziffer und Nährstoffkennziffer zusammen.

Standortstyp: „Der Standortstyp ist die kleinste kartierte ökologische Einheit, eine Zusammenfassung von Einzelstandorten, welche die gleichen Auswirkungen auf das Waldwachstum und die gleichen waldbaulichen Möglichkeiten und Gefahren aufweisen." (Niedersächsische LandesforstVerwaltung 1987, S. 29 A). In dieser Arbeit wird der Standortstyp über die Standortskennziffer* definiert. 
Stärkeklassen: „Einteilung der Stämme nach Brusthöhendurchmesserstufen in stehenden Beständen bzw. nach Stammholz-Mittendurchmesser des geernteten Holzes. Die Gliederung nach BHD*-Stärkeklassen gibt Aufschluß über die Struktur, Aufbauform und Wert von Beständen." (Niedersächsische LandesforstVerwaltung 1987, S. 30 A)

Unterabteilung: „Teilflächen einer Abteilung, die wegen erheblicher Bestandes- oder Standortsverschiedenheiten gegenüber anderen Teilflächen des Holzbodens* oder Nichtholzbodens* dauernd unterschiedliche Betriebsmaßnahmen erfordern." (Niedersächsische LandesforstVerwaltung 1987, S. 31 A)

Verbleibender Bestand: „Gesamtheit der Bäume, die bei einem Durchforstungseingriff stehen bleiben." (Bonnemann 1967)

Vfm: siehe „Vorratsfestmeter“*

Vornutzung: „Die Holzernte auf dem Weg der Durchforstung.“ (Bonnemann 1967)

Vornutzungsmasse: In dieser Arbeit definiert als summarisches Volumen der Bäume, die innerhalb eines Eingriffs entnommen wurden, der durch ein Maßnahmenelement* des Typs „Durchforstung“ definiert ist.

Vorrat: „Der Vorrat je ha und im Ganzen gibt das Volumen des stehenden Bestandes im allgemeinen in Kubikmeter mit Rinde <...> an." (KRAMER Und AKCA 1982)

Vorratsfestmeter (Vfm): „Maßeinheit für den Holzvorrat und Zuwachs* in Kubikmetern Derbholz ${ }^{*}$ mit Rinde. $1 \mathrm{Vfm}=0,8 \mathrm{Fm}^{*}, 1 \mathrm{Fm}^{*}=1,25 \mathrm{Vfm}$ “ (NiedersÄCHSISCHE LANDESFORSTVERWALTUNG 1987, S. 33 A)

Waldbau: „Die planmäßige Bewirtschaftung des Waldes, insbesondere die Maßnahme der Pflanzenzucht, der Walderneuerung, der Bestandeserziehung und Pflege sowie der Bodenmeloration in der forstlichen Praxis." (Bonnemann 1967)

Waldentwicklungstyp: Planerischer Entwicklungstyp eines Bestandes*. Ein Waldentwicklungstyp definiert sich über ein Leitbild in dem u.a. die angestrebte Struktur und die sukzessionale Stellung des WET festgehalten sind. Die Waldentwicklungsziele eines WET beziehen sich auf Zielstärken*, vorgesehene Schutz- und Erholungselemente, Baumartenanteile und Verjüngungsziele. Die Ergebnisse der Standortskartierung, die Waldbauregion und die waldbauliche Ausgangslage bestimmen die WET-Wahl. (siehe Niedersächsische LandesforstVerwaltung 2004)

WET: siehe „Waldentwicklungstyp“*

Z-Baum: siehe „Zukunftsbaum“*

Ziel-Dg: Siehe „Zieldurchmesser“* (auf den Bestand* bezogen). Rahmenwert* der Simulation

Zieldurchmesser: „Angestrebter Mindestdurchmesser in Brusthöhe eines Baumes oder angestrebte mittlere Mindestdurchmesser eines Bestandes*." (Bonnemann 1967)

Zielerreichungsprozent: Ausgabeparameter der Simulation. Definition siehe Seite $86 \mathrm{ff}$.

Zielstärke: Siehe „Zieldurchmesser“* (auf den Baum bezogen). Rahmenwert* der Simulation.

Zieltyp: In dieser Arbeit wird unter dem Zieltyp der angestrebte Typ des Bestandes* verstanden, der sich durch die Festlegung der Baumarten-Mischungsanteile definiert. Je nach Strategie werden unterschiedliche Zieltypen angestrebt.

Zukunftsbaum: Ein Baum von im Bestand* überdurchschnittlicher Qualität, dessen Zuwachs* durch Freistellung unterstützt wird. Im Idealfall wird er Teil des Endbestandes (DENGLER 
1992 в). Innerhalb der Simulation werden Bäume je nach Strategie als Z-Bäume ausgewählt, um sicherzustellen, daß sie bei Durchforstungsmaßnahmen bevorzugt freigestellt werden. Eine permanente Markierung definiert den Baum dauerhaft als Endbestandesanwärter. Eine temporäre Markierung wird nach der Durchforstungsmaßnahme gelöscht. Sie dient lediglich dazu, die Durchforstung strukturiert ablaufen zu lassen.

Zuwachs: Jährlicher Holzzuwachs eine Waldes je Hektar (NFV UND NFA 2002). Ausgabeparameter der Simulation. Definition siehe Seite $86 \mathrm{ff}$. 


\section{Lebenslauf}

Name: Henriette Abigail Aline Duda

Geboren: $\quad 22.12 .77$ in Köln

Nationalität: deutsch

Familienstand: ledig

08/84 - 07/88 Grundschule

Ev. Grundschule „Am Broich“, Bergisch Gladbach

08/88- 06/97 Gymnasium

Nicolaus-Cusanus-Gymnasium, Bergisch Gladbach

Abschluß: Abitur

08/97 - 05/98 Diakonisches Jahr

Habo (Schweden)

10/98 - 04/02 Bachelorstudiengang Forstwissenschaft

Georg-August-Universität, Göttingen

Abschluß: Bachelor

04/02 - 06/02 University of California, Berkeley (USA), Praktikum

Departement of Environmental Science Policy and Management, Devision of Ecosystem Sciences

05/02 - 02/03 Masterstudiengang Forstwissenschaft

Georg-August-Universität, Göttingen

Schwerpunkt: Waldökosystemanalyse und Informationsverarbeitung Abschluß: Master

05/03 - 06/06 Wissenschaftliche Mitarbeit, Promotion

Nordwestdeutsche Forstliche Versuchsanstalt, Göttingen

Drittmittelprojekte zu den Themen:

„Integrierte Bewertung biologischer Vielfalt am Beispiel des ökologischen Waldumbaus"

„Decision Support System Waldfunktionen - Entwicklung eines Decision Support Systems für die betriebliche und regionale Waldbauplanung“ 



\section{Vergleich forstlicher Managementstrategien}

Umsetzung verschiedener Waldbaukonzepte in einem Waldwachstumssimulator

Waldbesitzer stehen vor der planerischen Herausforderung, ihren Wald so zu bewirtschaften, daß die vielschichtigen Ziele einer multifunktionalen Forstwirtschaft umgesetzt werden können. Nicht nur die Nutzfunktion, sondern auch die Schutz- und Erholungsfunktion sollen erhalten bleiben. Um auf sachlicher Ebene Entscheidungen treffen zu können, ist es wichtig, die langfristigen Auswirkungen von Waldbewirtschaftungsmaßnahmen zu kennen. Das Ziel dieser Dissertation ist es, mit einem geeigneten Instrument die Transparenz über den zu erwartenden Zustand von Wäldern zu erhöhen.

In der vorliegenden Veröffentlichung wird eine Möglichkeit vorgestellt, Waldbaukonzepte in einem Waldwachstumssimulator (BWinPro) abzubilden.Vier forstliche Managementstrategien wurden exemplarisch in den bestehenden Programmcode integriert: 1. eine ertragsorientierte Alternative (ERTRAG), 2. ein Ökonomie und Ökologie vereinendes Konzept (LÖWE), 3. die Umsetzung einer naturnaher Bestockung (PNV) und 4. eine kaum steuernd eingreifende Variante (PROZESS). Als Input dienen gängige Forsteinrichtungsdaten aus einer relationalen Datenbank.

Beispielhaft wird die vierzigjährige Entwicklung einzelner Bestände und eines Untersuchungsgebiets in der Lüneburger Heide simuliert. Anhand von Nachhaltigkeitsparametern werden die Auswirkungen der unterschiedlichen Behandlungen dargestellt und vergleichend diskutiert.

Diese Dissertation entstand in den Jahren 2003 bis 2006 an der Nordwestdeutschen Forstlichen Versuchsanstalt in Göttingen. Sie wurde an der Fakultät für Forstwissenschaften und Waldökologie der Universität Göttingen eingereicht und ist auch unter folgender Internetadresse veröffentlicht:

http://resolver.sub.uni-goettingen.de/purl/?webdoc-1300 